\title{
Eastern Palearctic oak cynipid inquilines \\ (Hymenoptera: Cynipidae: Synergini) and associated gallwasps (Hymenoptera: Cynipidae: Cynipini) \\ Ph.D. thesis
}

Schwéger Szabina

Supervisors:

Dr. Pénzes Zsolt

Dr. Melika George

University of Szeged, Faculty of Science and Informatics

Doctoral School of Environmental Sciences

Department of Ecology

2016

Szeged 


\section{CONTENTS}

\section{INTRODUCTION}

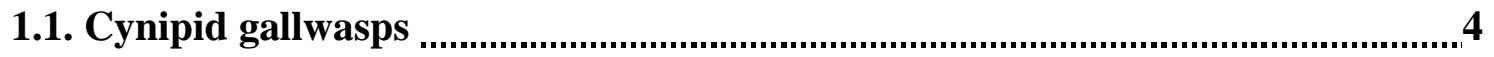

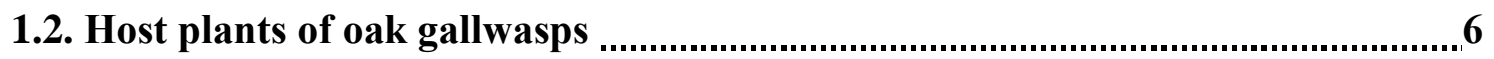

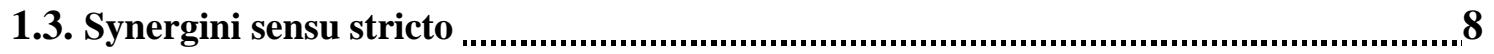

1.4. Origin and evolution of cynipid inquilines; phylogeny of Synergini sensu sricto 11

1.5 Eastern Palearctic oak gallwasps (Cynipidae: Cynipini) ....................................12

1.6. Main objectives of the work .............................................................................

\section{MATERIALS AND METHODS}

2.1. Synergini sensu stricto .....................................................................................

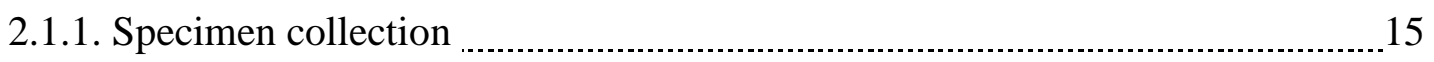

2.1.2. DNA extraction, sequencing, alignment and phylogenetics _........................ 15

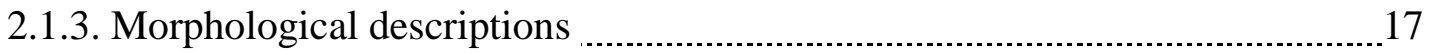

2.2. Oak gallwasps (Cynipidae: Cynipini) ………………........................................17

\section{RESULTS}

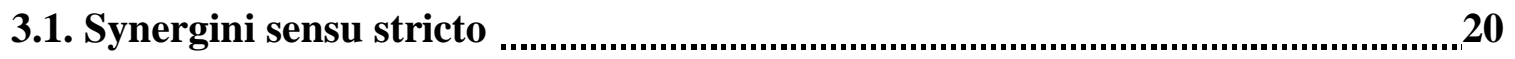

3.1.1. Key to Cynipidae tribes

3.1.2. Key to Synergini sensu stricto genera

3.1.3. Agastoroxenia Nieves-Aldrey \& Medianero, $2010 \ldots 30$

3.1.4. Lithosaphonecrus Tang, Melika \& Bozsó, 2013 _..................................... 30

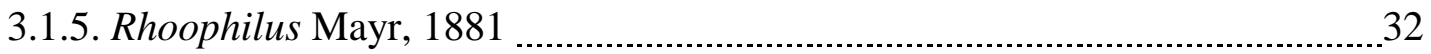

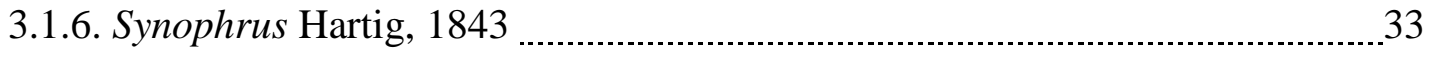

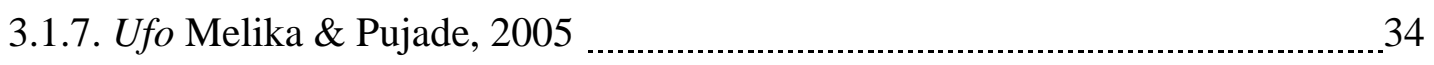

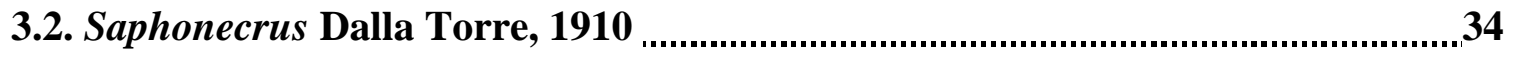

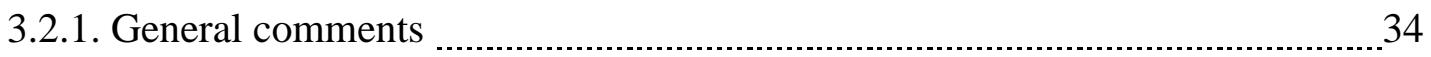

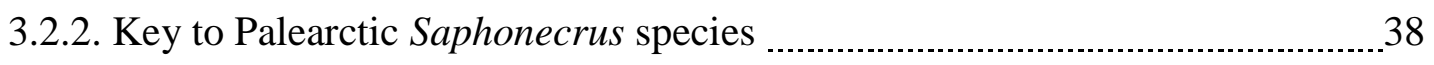

3.2.3. Annotated List of worldwide Saphonecrus species …............................ 48 


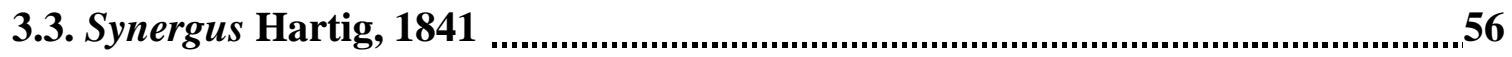

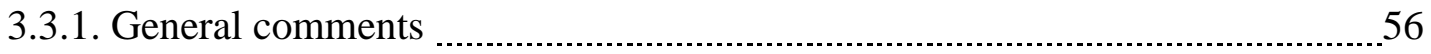

3.3.2. Key to Eastern Palearctic Synergus species …….................................... 58

3.3.3. Annotated List of Eastern Palearctic Synergus species ……............................. 63

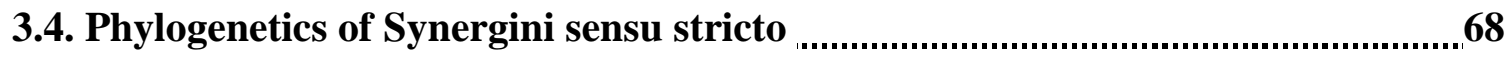

3.5. Eastern Palearctic Cynipini ........................................................................... 72

3.5.1. Eastern Palearctic oak gallwasps ……………………………………....... 72

\section{DISCUSSION}

4.1. Integrative taxonomy and phylogeny of Saphonecrus and Synergus species .....74

4.2. Phylogenetics of Synergini sensu stricto ……...................................................

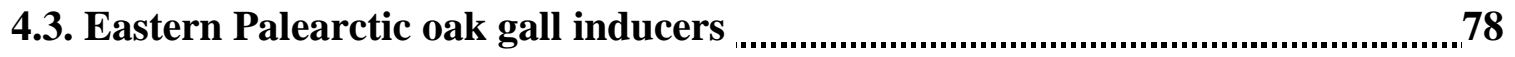

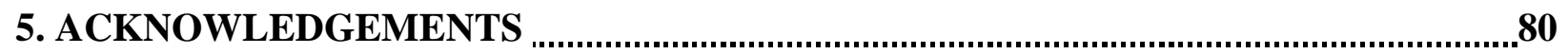

6. REFERENCES

7. SUMMARY

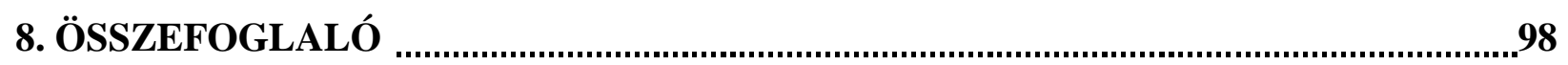

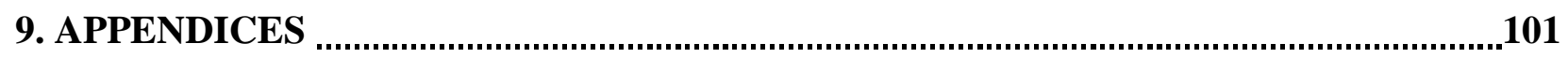

9.1. Morphological peculiarities and hosts of Lithosaphonecrus, Rhoophilus, Synophrus,Ufo, Saphonecrus and Synergus .........................................................102

9.2. Annotated list of Cynipini of the Eastern Palearctic (Cynipoidea: Cynipidae)..117

9.3. Eastern Palearctic Cynipini species with uncertain status 150 


\section{INTRODUCTION}

\subsection{Cynipid gallwasps}

Cynipidae lies within the superfamily Cynipoidea of the Hymenoptera, which includes approximately 3,000 described species (Fergusson 1995, Ronquist 1999). With the exception of the Cynipidae and some gall-inhabiting inquiline genera in Figitidae, the cynipoids are all parasitoids, and previous studies suggested that the cynipid gallwasps have evolved from parasitoid ancestors (Ronquist 1994, 1999). Cynipid gallwasps (Hymenoptera: Cynipidae) induce some of the world's most visually striking, and structurally complex plant galls. Approximately 1,440 cynipid gallwasp species were recognized (Liljeblad \& Ronquist 1998, Ronquist 1999, Ronquist \& Liljeblad 2001, Nieves-Aldrey 2001, Melika 2006), increased considerably recently, although Nordlander (1984) has estimated that the actual number is between 3,000 and 6,000. Ronquist \& Liljeblad (2001) hypothesized that the gallwasps arose in Eurasia, around the Black Sea, and that the genera Eschatocerus (gall inducers on Acacia and Prosopis) and Rhoophilus (inquilines on Rhus galls) spread later to South America and South Africa, respectively. However, recent results may suggest alternative hypothesis, and as such the biogeographical history of the basal Cynipidae is still not clear (Nylander et al. 2004, Ronquist et al. 2015).

Cynipids are divided into two main trophic groups: the gall inducers, and the gallassociated inquilines. Inquilines develop in galls but can not induce them, except of few Synergus species have not lost the capability to induce galls (Csóka et al. 2005). In general, inquilinism is a form of cleptoparasitism, usually considered to represent a unilaterally beneficial relationship that benefits only the inquiline (Askew 1984, Ronquist 1994, 1999). This biological division is moderately reflected in the higher-level taxonomy of Cynipidae that has been changed recently. Instead of 8 tribes recognized earlier, the family is divided into 12 tribes (Ronquist et al. 2015) (Table 1).

Most of the described species of Cynipidae are gall inducers (Table 1.). However, more than 220 species, classified traditionally into ten genera and placed to four tribes recently (Table 1), develop as inquilines inside galls of other cynipids (Pujade-Villar et al. 2003, Nieves-Aldrey \& Medianero 2010, Bozsó 2015, Bozsó et al. 2014, 2015, Ronquist et al. 2015). Inquiline cynipids feed obligately on plant tissues within developing galls, and to some extent, stimulate 
the development of tissues characteristic to galls. Our research focuses particularly on inquilines that attack hosts in the Cynipini (oak gall wasps) associated to Fagaceae host plants. Some synthesis based on published data has also been done on oak gall inducers.

Table 1. Classification, diversity and host associations of Cynipidae (after Ronquist et al. 2015, genera and species numbers updated by Sz. Schwéger)

\begin{tabular}{|c|c|c|c|c|}
\hline Tribes & Genera & Species & Hosts & Distribution \\
\hline Cynipini & 41 & 954 & $\begin{array}{c}\text { Gall inducer on Fagaceae (Quercus, } \\
\text { Castanea, Castanopsis, } \\
\text { Chrysolepis and Lithocarpus) }\end{array}$ & Holarctic, Neotropical, Oriental \\
\hline Diplolepidini & 2 & 55 & Gall inducer on Rosa (Rosaceae) & Holarctic \\
\hline Pediaspidini & 2 & 2 & Gall inducer on Acer (Aceraceae) & Palearctic \\
\hline Eschatocerini & 1 & 3 & $\begin{array}{c}\text { Gall inducer on Acacia, Prosopis } \\
\text { (Fabaceae) }\end{array}$ & South Neotropical \\
\hline Qwaqwaiini & 1 & 1 & $\begin{array}{c}\text { Gall inducer on } \\
\text { Scolopia spp. (Salicaceae) }\end{array}$ & Afrotropical (South Africa) \\
\hline Paraulacini & 2 & 6 & $\begin{array}{c}\text { Inquilines or parasitoids in chalcidoid } \\
\text { galls (Pteromalidae) on } \\
\text { Nothofagus spp. } \\
\text { (Nothofagaceae) } \\
\end{array}$ & South Neotropical \\
\hline $\begin{array}{l}\text { Aylacini } \\
\text { sensu stricto }\end{array}$ & 3 & 9 & $\begin{array}{c}\text { Gall inducers on Papaver spp. } \\
\text { (Papaveraceae) }\end{array}$ & Palearctic \\
\hline Aulacideini & 9 & 78 & $\begin{array}{c}\text { Gall inducers on Asteraceae, Lamiaceae, } \\
\text { Valerianaceae and the tribe } \\
\text { Fumarioideae (Papaveraceae) }\end{array}$ & Holarctic \\
\hline Phanacidini & 4 & 34 & $\begin{array}{c}\text { Gall inducers on several genera of } \\
\text { Asteraceae, rarely on Phlomis } \\
\text { (Lamiaceae) and Eryngium } \\
\text { (Apiaceae) }\end{array}$ & $\begin{array}{c}\text { Palearctic, one species South } \\
\text { Afrotropical (possibly introduced) } \\
\text { Introduced in South America } \\
\text { and Australia } \\
\end{array}$ \\
\hline Diastrophini & 4 & 43 & $\begin{array}{l}\text { Gall inducers in galls on Rubus spp. and } \\
\text { Potentilla spp. (Rosaceae), rarely on } \\
\text { Smilax (Smilacaceae), and inquilines } \\
\text { in cynipid galls on Rubus spp. } \\
\text { Synophromorpha) and Rosa spp. } \\
\text { (Rosaceae) (Periclistus) }\end{array}$ & $\begin{array}{l}\text { Holarctic and Neotropical } \\
\text { (one species) }\end{array}$ \\
\hline Ceroptresini & 1 & 24 & $\begin{array}{l}\text { Inquilines in galls induced by Cynipini, } \\
\text { one genus, Ceroptres. }\end{array}$ & Holarctic \\
\hline $\begin{array}{l}\text { Synergini } \\
\text { sensu stricto }\end{array}$ & 7 & 155 & \begin{tabular}{|c|} 
Inquilines in cynipid galls on \\
Fagaceae genera, Quercus \\
(Agastoroxenia, Lithosaphonecrus, \\
Saphonecrus, Synophrus, Synergus, \\
Ufo). One genus (Rhoophilus) is an inquiline \\
Cecidosidae (Lepidoptera) galls on \\
Rhus spp. (Anacardiaceae) \\
\end{tabular} & $\begin{array}{c}\text { Holarctic, Neotropical, Oriental, } \\
\text { Oceanian }\end{array}$ \\
\hline Cynipidae & 77 & 1364 & & \\
\hline
\end{tabular}




\subsection{Host plants of oak gallwasps}

Oak gallwasps, inducers and inquilines, are intimate parasites of trees belong to the familiy Fagaceae. In order to understand their evolution and recent distribution the knowledge of their host plant associations is important. Fagaceae is divided into two subfamilies: Fagoidea, with Fagus and Quercus genera, and Castaneoidea, with insect-pollinated Castanea, Castanopsis, Chrysolepis, Lithocarpus and Notholithocarpus, all five of which serve as hosts for Cynipini (Govaerts \& Frodin 1998, Manos et al. 2008). The number of known species within Fagaceae is contraversial; from 900 to $\mathrm{ca}$. 1,050 species are mentioned (Govaerts \& Frodin 1998, Oh \& Manos 2008). No gallwasps are known from some Fagaceae genera. The family Fagaceae formerly included the southern beeches of the genus Nothofagus distributed in South America and Australasia (Li et al. 2007). Though no Cynipini attack Nothofagus, they have been colonised by the gallwasp genera Paraulax Kieffer and Cecinothofagus Nieves-Aldrey \& Liljeblad documented in Chile and Argentina, however no Cynipidae are known on this genus from Australasia (Nieves-Aldrey et al. 2009). Gallwasps are mainly associated to the following six genera of Fagaceae.

Castanea (chestnuts) genus is represented by 8-10 species, native to temperate regions of the Northern Hemisphere, with two known gallwasp species, Dryocosmus kuriphilus and D. zhuili (Zhu et al. 2015). No cynipid inquilines were reared yet from these galls, however, recently Synergus castaneus was described from China as the first cynipid inquiline species associated with Castanea galls (Bernardo et al. 2013).

Castanopsis (chinquapin oaks) is a genus which involves about 120 species, all of which are restricted to eastern Asia (Oh \& Manos 2008). Recently a number of new gallwasp and cynipid inquiline species were described from Castanopsis (Tang et al. 2016a, Schwéger et al. 2015a, b).

Chrysolepis (golden chinquapin oaks) is a small genus, endemic to the western United States, with two species, Ch. chrysophylla and Ch. sempervirens. Few inquilines associate with few cynipid gallwasps develop on Chrysolepis are known (Burks 1979).

Lithocarpus (stone oaks) is a large genus, distributed in east and southeast Asia, with more than 300 species (Govaerts \& Frodin 1998). Recently this genus has been shown to host Cynipini (Tang et al. 2011a) and cynipid inquilines (Bozsó et al. 2014).

Notholithocarpus (tanbark oaks or tanoaks) with only one known North American species, N. densiflorus, only recently was established (Govaerts \& Frodin 1998, Manos et al. 2008, Oh \& 
Manos 2008). It is an evergreen tree, native to the western United States. It is most closely related to the north temperate oaks, Quercus, but not to the Asian Lithocarpus. Few cynipids are known to associate with this host (Burks 1979) including inquilines (Bozsó et al. 2015).

Majority of Cynipini induce galls on members of a large genus Quercus which is divided into two long-established subgenera - the strictly Asian subgenus Cyclobalanopsis and the more widespread subgenus Quercus (Camus 1936-54, Nixon 1989, 1993, Govaerts \& Frodin 1998, Manos et al. 1999). Many of them are serving as host for inquilines, too. The subgenus Quercus is divided into discrete sections (Govaerts \& Frodin 1998), including Lobatae (the red oaks), Protobalanus (the golden cup or intermediate oaks), Quercus sensu stricto (the white oaks), and Cerris. The latter comprise the Ilex group, too. Of the 4 oak sections in the subgenus Quercus, Quercus sensu stricto is Holarctic, Lobatae and Protobalanus are restricted to North America, and Cerris is restricted to the Palearctic. Worldwide, there are 531 recognised oak species (according to Govaerts \& Frodin 1998), but generally reported as between 300 and 600 (Hubert et al. 2014). This total is divided between the Southeast Asian subgenus Cyclobalanopsis (76 species), and the more widespread subgenus Quercus, with 455 species. The subgenus Quercus is most abundant in temperate regions of the Northern Hemisphere. In the Neotropics oaks extend southwards as far as the Colombian Andes (Nieves-Aldrey 2005). The Palearctic supports ca. 170 species, with $c a .130$ species in the EP and only 29 species in the WP (Govaerts \& Frodin 1998). The oak sections Cerris and Quercus sensu stricto are widespread in the EP, and the regional richness of oaks - with 32 species in China (Linkuo \& Tao 1998), at least 17 in Himalayan India, Nepal and Bhutan (Negi \& Naithani 1995), and 6 in Japan (Ohwi 1961) exceeds the Western Palearctic's 29 species.

Oaks and their close relatives probably first diversified in Southeast Asia, either during the Palaeocene (65-56 mya) or the Eocene (56-35 mya) (Zhou 1992, 1993, Cannon \& Manos 2003, Manos et al. 1999), with an ancient divide into two monophyletic lineages: (i) the subgenus Cyclobalanopsis and the section Cerris of the subgenus Quercus and (ii) sections Lobatae, Quercus sensu stricto and Protobalanus of the subgenus Quercus (Manos et al. 2001), althought the placement of Cyclobalanopsis is equivocal (Hubert et al. 2014). The Asian distribution of Cyclobalanopsis, the Eurasian distribution of section Cerris, and the absence of fossils of these two groups from the Nearctic suggest that oaks originated and differentiated into these two basal lineages in Asia (Zhou 1992, 1993, Manos \& Stanford 2001). 
A striking feature of phylogenetic analyses of the oak gallwasps (Ács et al. 2007, Stone et al. 2009) is that (with the exception of host alternator species) they show the same deep divide between genera associated with section Cerris on one hand, and those associated with sections Quercus sensu stricto and the nearctic red oak section Lobatae on the other. If the divergence between the oak sections occurred in Eastern Asia, then it is plausible that this same region was the cradle for the origin and initial diversification of oak gallwasps and their inquilines. Nevertheless, althought there are clear host specific clades (Ács et al. 2010, Pénzes et al. 2012, Bozsó et al. 2014), the general pattern is to be explored.

\subsection{Synergini sensu stricto}

According to the earlier classifications, Synophromorpha Ashmead, Periclistus Förster, Ceroptres Hartig, Agastoroxenia Nieves-Aldrey \& Medianero, Lithosaphonecrus Tang, Melika \& Bozsó, Saphonecrus Dalla Torre \& Kieffer, Synophrus Hartig, Synergus Hartig, Ufo Melika \& Pujade and Rhoophilus Mayr, were classified within the polyphyletic tribe Synergini (Burks 1979, Ronquist 1999, Pénzes et al. 2012, Ronquist et al. 2015). A world-wide review of all cynipid inquilines was given in Pénzes et al. (2012). Recently, Periclistus and Synophromorpha were moved to the newly established Diastrophini tribe; a new tribe Ceroptresini was established for the Ceroptres genus, while the rest 7 genera, Agastoroxenia, Lithosaphonecrus, Saphonecrus, Synophrus, Synergus, Ufo, Rhoophilus, were put into the tribe Synergini sensu stricto (Ronquist et al. 2015, Table 1).

Phylogeny of the oak gallwasp inquilines, based mainly on the Eastern and Western Palearctic species, has long been controversial, especially in the Synergini sensu stricto genera (Ács et al. 2010). Table 2 shows the number of described species including all the newly described Synergus and Saphonecrus (Schwéger et al. 2015a, b; Results section).

The number of known Synergini species is unevenly distributed among the zoogeographical regions (Table 2). This clearly reflects the differences in the intensity of sampling. Western Palaeartic (WP) is the best known. The Synergini fauna of Eastern Palearctic (EP) is being explored recently. It is expected to be more diverse and probably many new species will be described in the future. In part, this expectation can be explained by the diversity of potential host plant (detailed below), compared to the WP. Tropical regions are poorly studied. 
Table 2. The world distribution and species richness of Synergini sensu stricto genera (WP, Western Palearctic, EP, Eastern Palearctic, OR, Oriental Region, NA, Nearctic, NT, Neotropical Region, ET, Ethiopian Region).

\begin{tabular}{|l|l|l|l|l|l|l|l|l|}
\hline Genera & Hosts & WP & EP & OR & NA & NT & ET & World \\
\hline $\begin{array}{l}\text { Agastoroxenia } \\
\text { Nieves-Aldrey\& Medianero, } \\
\text { 2010 }\end{array}$ & $\begin{array}{l}\text { Oak cynipid } \\
\text { galls }\end{array}$ & - & - & - & - & 1 & - & 1 \\
\hline $\begin{array}{l}\text { Lithosaphonecrus Tang, } \\
\text { Melika \& Bozsó, 2013 }\end{array}$ & $\begin{array}{l}\text { Oak cynipid } \\
\text { galls }\end{array}$ & - & 4 & 2 & - & - & - & 6 \\
\hline $\begin{array}{l}\text { Synergus } \\
\text { Hartig, 1840 }\end{array}$ & $\begin{array}{l}\text { Oak cynipid } \\
\text { galls }\end{array}$ & 30 & 19 & - & 55 & 14 & - & 118 \\
\hline $\begin{array}{l}\text { Saphonecrus } \\
\text { Dalla Torre \& Kieffer, 1910 }\end{array}$ & $\begin{array}{l}\text { Oak cynipid } \\
\text { galls }\end{array}$ & 6 & 25 & 2 & 3 & - & - & 36 \\
\hline $\begin{array}{l}\text { Synophrus } \\
\text { Hartig, 1843 }\end{array}$ & $\begin{array}{l}\text { Oak cynipids } \\
\text { galls }\end{array}$ & 7 & - & - & - & - & - & 7 \\
\hline $\begin{array}{l}\text { Ufo } \\
\text { Melika \& Pujade, 2005 }\end{array}$ & $\begin{array}{l}\text { Oak cynipid } \\
\text { galls }\end{array}$ & - & 3 & 1 & - & - & - & 4 \\
\hline $\begin{array}{l}\text { Rhoophilus } \\
\text { Mayr, 1881 }\end{array}$ & Rhus galls & - & - & - & - & - & 1 & 1 \\
\hline Total: & & $\mathbf{4 3}$ & $\mathbf{4 8}$ & $\mathbf{5}$ & $\mathbf{5 8}$ & $\mathbf{1 5}$ & $\mathbf{1}$ & $\mathbf{1 7 0}$ \\
\hline
\end{tabular}

Inquilines which attack hosts in the gallwasp tribe Cynipini (gall inducer oak gallwasps) include six genera, Agastoroxenia, Saphonecrus, Synergus, Synophrus, Ufo, Lithosaphonecrus, earlier named as the Synergus complex of genera (Pénzes et al. 2012). One genus, Rhoophilus, with one species, $R h$. loewi Mayr, known from South Africa, is an inquiline in Cecidosidae (Lepidoptera) galls on Rhus spp. (Anacardiaceae). It was recently examined in details (van Noort et al. 2007). Probably all seven genera form together a distinct monophyletic lineage (Ronquist et al. 2015) and Rhoophilus generally recovered as the sister taxon of all others (e.g. Ács et al. 2010, Ronquist et al. 2015). The morphological taxonomy of Synergus complex has been studied in details (Mayr 1872; Wiebes-Rijks, 1979; Nieves-Aldrey \& Pujade-Villar 1985, 1986; PujadeVillar \& Nieves-Aldrey 1990, 1993; Liljeblad \& Ronquist 1998; Liljebald et al. 2008; PujadeVillar et al. 2003, Melika 2006; Melika et al. 2012; Bozsó et al. 2015) and (i) supported the differentiation of these two lineages, and (ii) showed that five genera, Agastoroxenia, Saphonecrus, Synergus, Synophrus and Ufo morphologically differs from Ceroptres .

Inconsistencies in the taxonomy of gallwasps were revealed by the DNA sequence-based studies. For example the presence of cryptic species, independently evolving lineages that are morphologically indistinguishable, had been demonstrated in many times (Ács et al. 2010, Kaartinen et al. 2010). Consequently it is becoming increasingly common to supplement morphological identifcation with molecular methods such as DNA barcoding (Hebert et al. 
2003). Recently obtained data suggest that the variability of the widely-used region of the mitochondrial coxI gene together with $c y t b$ and the nuclear 28S D2 sequences can be used to define clusters of samples (MOTU, Molecular Operational Taxonomic Unit, Blaxter et al. 2005.) within the Synergus complex of genera (Ács et al. 2010). However, comparison of morphologybased species identification with MOTU designations revealed substantial discordance (explained below). The application of morphological and molecular characters together helped us to establish a more reliable taxonomy for Synophrus (Pénzes et al. 2009), Synergus (Ács et al. 2010), Ufo (Melika et al. 2012), Saphonecrus (Bozsó et al. 2014) and Lithosaphonecrus (Bozsó 2015, Bozsó et al. 2015).

\section{Synergus Hartig 1941}

Thirty Synergus species are known from the Western Palearctic (Pujade-Villar et al. 2003, Pénzes et al. 2012), which from about $80 \%$ of species was involved into a DNA sequencebased delimitation and phylogenetic analysis (Ács et al. 2010). The analyses supported monophyly of the genus Synergus but rejected the traditional classification, namely the monophyly of the two sections within Synergus (Section I and Section II, Mayr 1872), and the monophyly of some morphospecies (S. hayneanus, S. pallipes, S. umbraculus, S. flavipes). The results showed that some distinct WP Synergus morphospecies belong to the same MOTU, while some morphospecies, e.g. Synergus umbraculus, S. hayneanus, revealed cryptic species. In other words some recognised WP Synergus species correspond to MOTUs, others clearly do not. The sequence data was unable to discriminate between $S$. gallaepomiformis, S. pallicornis, $S$. pallidipennis and S. pallipes. All these results suggested that some WP Synergus morphospecies should be revised.

Before our work 12 valid species of Synergus were known from the Eastern Palearctic (Sadeghi et al. 2006, Bernardo et al. 2013, Abe et al. 2011, Pujade-Villar et al. 2014a, b, c). While all known WP Synergus species associate with galls on Quercus (subgen. Quercus sections Quercus and Cerris) precise host associations were known only for a few Eastern Palearctic Synergus species before 2008 (Abe 1990, 1992a, Abe et al. 2007). Large number of Synergus specimens was reared during 2008-2012 from different hosts which associate with Quercus and Quercus related genera within Fagaceae. The taxonomic assignment of undescribed species from Japan, the Far East Russia and Taiwan and the re-appraisal of known EP species 
with a preliminary phylogenetic placement has been done (Schwéger et al. 2015a). However, taxonomic status of Nearctic Synergus is still equivocal (Pénzes et al. 2012).

Saphonecrus Dalla Torre, 1910 and Lithosaphonecrus Tang, Melika \& Bozsó, 2013

Our knowledge about the taxonomic diversity of Saphonecrus has been expanded recently, especially in the EP region (Table 2). Before 200824 species of Saphonecrus were known worldwide covering the Palearctic, Nearctic and the Oriental region (Pénzes et al. 2012, Bozsó et al. 2014). The six WP species are associated mainly with galls on section Cerris oaks, including Mediterranean evergreen oaks (Quercus ilex L., Q. suber L., Q. coccifera L.) and $Q$. cerris L. in Central Europe, while some are associated with galls that develop on white oaks (section Quercus, e.g. Q. petraea Liebl., Q. robur L. in Central Europe (Schwéger et al. 2015b). In 2007, seven Saphonecrus species were listed for the EP (Abe et al. 2007) and two species, $S$. serratus Weld and S. areolatus Weld, were known from the Oriental Region (Weld 1926). Recently new species were described from Japan and China (Liu et al. 2012, Wang et al. 2010, Wachi et al. 2011b, Pujade-Villar et al. 2014a, c). The most complete molecular phylogenetic reconstruction of Saphonecrus with examination of known species was recently proposed with involving new lineages from Japan, Russia, China and Taiwan (Pénzes et al. 2012, Melika et al. 2012, Bozsó et al. 2014, 2015). The taxonomic assignment of new species and the re-appraisal of all known Saphonecrus species worldwide based on morphological and molecular characters was evaluated by us (Schwéger et al. 2015b). Four Saphonecrus species listed for the Nearctic (Burks 1979), they possess some non-typical character states for Saphonecrus, and thus their taxonomic assignment is questioned.

New genera, Lithosaphonecrus Tang, Melika \& Bozsó and Lithonecrus Nieves-Aldrey \& Butterill, and number of new species within genera of Synergini have recently been described (Bozsó et al. 2014, 2015; Melika et al. 2005, 2007, 2012; Pénzes et al. 2009; Nieves-Aldrey \& Butterill 2014) which were examined in details by us and as the result some genera and species were synonymized (Schwéger et al. 2015b).

\subsection{Origin and evolution of cynipid inquilines; phylogeny of Synergini sensu stricto}

Ronquist et al. (2015) distinguished three major possible scenarios for the origin of cynipid inquilines. Several previous studies suggested the "gall inducers first" scenario wherein 
the inquilines evolved once from herb gallers in the Northern Hemisphere. At first they lost the capability to induce galls and started to parasitize their closest relatives. Then, the inquilines radiated worldwide and parasitized several different Cynipini lineages. They found several phylogenetic evidences which shaded this hypothesis and they supported other scenario is called "multiple transitions" instead of the previous one. It concerned that the first gall inducer cynipids associated with woody hosts in the Northern Hemsiphere and after there have been a multiple transitions between cynipid gallers and inquilines. In this second scenario, inquilines do not form a monophyletic unit and transitions could be host plant specific (e.g. result in separate radiation on Rosaceae and Fagaceae). Third scenario called "inquiline first" wherein the ancestral gallwasp was inquiline, possibly in Southern Hemisphere and the gall inducers evolved independently multiple times in different lineages of inquilines. Further studies are needed worldwide to test these hypotheses.

The phylogeny of Synergini sensu stricto genera recently was investigated. The phylogenetic position of Synophrus and Ufo has been re-appraised and re-established (Bozsó et al. 2014, 2015; Melika et al. 2005, 2007, 2012; Pénzes et al. 2009; Nieves-Aldrey \& Butterill 2014).

\subsection{Eastern Palearctic oak gallwasps (Cynipidae: Cynipini)}

Oak gallwasps (Hymenoptera: Cynipidae: Cynipini) are by far the most species-rich group of gallwasps, with more than 1,000 known species worldwide (Csóka et al. 2005, Ronquist et al. 2015). There are only a few Cynipidae (Hymenoptera) reviews on the EP species (Dalla Torre \& Kieffer 1910, Abe et al. 2007). Both are out of time and since then dozens of new species were described and a large number of nomenclatorial changes have been done. Recently some review papers were published on EP cynipid inquilines (Cynipidae: Synergini) (Pénzes et al. 2012) and its genera in Synergini and Ceroptresini. Abe et al. (2007) listed all the known EP rose gallwasps (Diplolepidini) and herb gall wasps (“Aylacini”), while the list of oak gallwasps (Cynipini) currently is far from completeness. The Eastern Palearctic as defined here includes Asia east to Iran, the Ciscaucasus (Transcaucasus) and the Ural Mountains. Species known only from more western regions, such as from Iran, the Transcaucasus, Dagestan are not included.

Oak cynipid taxonomy and biology is in a period of rapid advance, both in terms of our understanding of cynipid biology and of resolving taxonomic issues within the Cynipini. New 
genera, species and generations continue to be discovered in Japan (Ide et al. 2010, 2012, 2013, Wachi \& Abe 2010), Taiwan (Melika et al. 2010, 2011, 2012, 2013, Tang 2015, Tang et al. 2009, 2011a, b, 2012a, b, 2016a, b, c), China (Pujade-Villar \& Wang 2012, Wang et al. 2013, Abe et al. 2014a, Pujade-Villar et al. 2014a), Vietnam (Abe et al. 2014b). In particular, Taiwan and China are areas with high oak (Quercus L., subgen. Cyclobalanopsis and Quercus) and oak relatives' (Castanea Miller, Castanopsis Miller, Lithocarpus Blume) species richness whose cynipid faunas remain little-studied, and future work will certainly reveal further new species.

Table 3. Species richness of the EP oak gallwasps (Cynipini) with their host associations

\begin{tabular}{|c|c|c|c|c|c|c|c|c|}
\hline Genus & 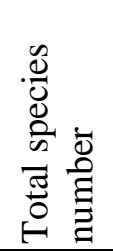 & 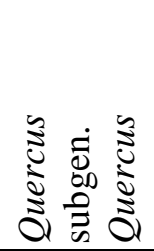 & 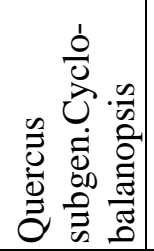 & 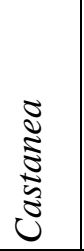 & 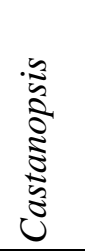 & 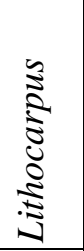 & 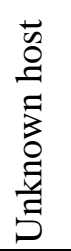 & 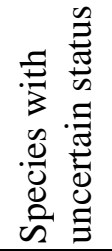 \\
\hline Andricus Hartig, 1840 & 16 & 16 & - & - & - & - & - & 2 \\
\hline Aphelonyx Mayr, 1881 & - & - & - & - & - & - & - & 2 \\
\hline Belizinella Kovalev, 1965 & 2 & 2 & - & - & - & - & - & - \\
\hline Biorhiza Westwood, 1840 & 1 & 1 & - & - & - & - & - & - \\
\hline Callirhytis Foerster, 1869 & 1 & 1 & - & - & - & - & - & 2 \\
\hline $\begin{array}{l}\text { Cerroneuroterus Melika \& Pujade- } \\
\text { Villar, } 2009\end{array}$ & 3 & 3 & - & - & - & - & - & - \\
\hline $\begin{array}{l}\text { Cyclocynips } \\
\text { Melika, Tang, \& Sinclair, } 2013\end{array}$ & 2 & - & 2 & - & - & - & - & - \\
\hline $\begin{array}{l}\text { Cycloneuroterus } \\
\text { Melika \& Tang, } 2011\end{array}$ & 17 & - & 15 & & 1 & 1 & - & - \\
\hline Cynips Linnaeus, 1758 & 1 & 1 & - & - & - & - & - & - \\
\hline Dryocosmus Giraud, 1859 & 12 & 2 & 2 & 2 & 4 & - & 2 & 2 \\
\hline Latuspina Monzen, 1954 & 9 & 9 & - & - & - & - & - & - \\
\hline Neuroterus Hartig, 1840 & 7 & 4 & 1 & - & - & - & 2 & 2 \\
\hline Plagiotrochus Mayr, 1881 & 7 & - & 5 & - & - & - & 2 & - \\
\hline Trichagalma Mayr, 1907 & 3 & 3 & - & - & - & - & - & - \\
\hline Trigonaspis Hartig, 1840 & 3 & 3 & - & - & - & - & - & - \\
\hline Ussuraspis Kovalev, 1965 & 1 & 1 & - & - & - & - & - & - \\
\hline TOTAL & 85 & 46 & 25 & 2 & 5 & 1 & 6 & 10 \\
\hline
\end{tabular}

All the data on the EP Cynipini fauna is fragmented, published in numerous taxonomic paper (Abe 2006, 2007, Abe et al. 2012, 2014a, b, Ide et al. 2010, 2012, 2013, Melika et al. 2010, 2012, 2013, Tang et al. 2009, 2011a, 2011b, 2016a, b, c). Thus our aim was to compile a list of all know EP Cynipini with including also species with uncertain status (nomena dubia). 
In the Western Palearctic and Nearctic the majority of oak gallwasps associate with oaks of the Quercus subgenus Quercus (Stone et al. 2002, Melika 2006). Recent studies in the Eastern Palearctic have revealed a potentially rich oak gallwasp fauna associate with four Fagaceae genera, Castanea, Castanopsis, Lithocarpus, Quercus (subgenus Quercus and subgenus Cyclobalanopsis) (Abe et al. 2014a, b, Ide et al. 2010, 2012, 2013, Melika et al. 2011, 2013, Tang et al. 2011a, b; Melika et al. 2011). Numbers of recognised species in each genus of Cynipini are summarised in Table 3. Different host plants were colonized by the representatives of different genera. For instance, species of Andricus, Belizinella, Biorhiza, Cerroneuroterus, Cynips, Latuspina, Trichagalma, Trigonaspis, and Ussuraspis, associate only with Quercus subgenus Quercus. Number of genera associate mainly with the species of Quercus subgenus Cyclobalanopsis and only few species of Cycloneuroterus and Dryocosmus are known to associate with other three oak related genera: two Dryocosmus species with Castanea, one Cycloneuroterus and four Dryocosmus species with Castanopsis, and one species of Cycloneuroterus with Lithocarpus.

\subsection{Main objectives of the work}

My aim is to summarize the taxonomic problems, concerning the EP Synergini and Cynipini fauna, using morphological and molecular data. The Main Objectives:

1. Taxonomic and phylogenetic re-appraisal of Synergini sensu stricto genera; synonymization of some genera and species (Pénzes et al. 2012, Schwéger et al. 2015a, b).

2. Combining keys for the identification of Synergini sensu stricto genera, with producing necessary colour plates with adequate morphological characters (Pénzes et al. 2012).

3. Composing first keys to all EP Synergus species and to Palearctic Saphonecrus species, with colour plates of adequate morphological characters (Schwéger et al. 2015a, b).

4. New EP species of Saphonecrus (15 new species were described by us) and Synergus (8 new species were described by us), their detailed diagnoses, descriptions, biology, host associations (Schwéger et al. 2015a, b).

5. Re-appraisal of all EP Synergus species and that of Palearctic Saphonecrus species (Schwéger et al. 2015a, b).

6. Compilation of the up-to-date complete list of EP Cynipini (unpublished result). 


\section{MATERIALS AND METHODS}

\subsection{Synergini sensu stricto}

\subsubsection{Specimen collection}

All the wasps we obtained were laboratory reared from fresh galls collected in different localities in Japan, Russia, China and Taiwan during 2008-2012 (coordinated by Graham N Stone, Univ. Edinburgh, UK). Galls were put into sealed plastic bags during the field work and taken to the laboratory for rearing. Galls collected in Japan, Russia and Taiwan during 2008 were reared at the University of Edinburgh, UK (reared by J. Nicholls); galls collected in later years in Taiwan and China were reared at the National Chung Hsing University, Taichuing, Taiwan (reared by C.-T. Tang). For rearing galls were placed in plastic containers at a room temperature, with square windows cut into the lids and covered with a mesh for the proper ventilation to avoid fungal infection. Containers were checked every day, and wasps that had emerged were aspirated and placed in $99 \%$ ethanol for further laboratory processing. All the reared wasps were sent to G. Melika (Plant Health and Molecular Biology Laboratory, National Food Chain Safety Office, Budapest) for further morphological identification. For the host plants identification Lu et al. (2006) and Govaerts \& Frodin (1998) were used.

\subsubsection{DNA extraction, sequencing, alignment and phylogenetics}

Adults were preserved in $99 \%$ ethanol and stored at $-20{ }^{\circ} \mathrm{C}$ until DNA extraction. Some specimens, including Synergus kawakamii were obtained as dry samples. Genomic DNA was extracted from one to three legs and/or the whole body of adult specimens, following the chelex extraction method presented in Nicholls et al. (2010). A fragment of the mitochondrial cytochrome c oxidase subunit I (coxI) gene was amplified using the forward primer LCO-1490 (5' GGT CAA CAA ATC ATA AAG ATA TTG G 3') and HCO-2198 (5' TAA ACT TCA GGG TGA CCA AAA AAT CA 3') (Folmer et al. 1994). A fragment of the D2 expansion segment of the nuclear $28 \mathrm{~S}$ ribosomal array (28S D2) was amplified using the primers D2 forward (5'CGTGTTGCTTGATAGTGCAGC-3') and D2 reverse (5'-TCAAGACGGGTCCTGAAAGT-3') (Hancock et al. 1988). Each $25 \mu \mathrm{l}$ PCR mix consisted of $0.25 \mu 1$ of $5 \mathrm{U} / \mu 1$ Taq DNA polymerase (Fermentas), $2.5 \mu \mathrm{l}$ of 10X Taq buffer, $2.0 \mu \mathrm{l} \mathrm{MgCl}_{2}(25 \mathrm{mM}), 0.5 \mu \mathrm{ldNTP}(10 \mathrm{mM}), 0.5 \mu 1$ of each primers $(20 \mathrm{pmol}), 2.0 \mu \mathrm{l}$ template DNA, and $16.75 \mu 1$ purified and distilled water. Cycling 
conditions were $94^{\circ} \mathrm{C}$ for 2 minutes, followed by 35 cycles of $94^{\circ} \mathrm{C}$ for 30 seconds, $45^{\circ} \mathrm{C}$ (28SD2) or $50^{\circ} \mathrm{C}(\operatorname{coxl})$ for 30 seconds, and $72^{\circ} \mathrm{C}$ for 45 seconds, with a final step of $72^{\circ} \mathrm{C}$ for 10 minutes. PCR products were purified from 1\% agarose gel using the Millipore Ultrafree-DA DNA extraction kit. PCR products were sequenced directly by MWG-Biotech AG (http://www.mwg-biotech.com) or LGC Genomics Gmbh using Sanger method. Chromatograms were checked by eye using Staden package 2.0 (Bonfield et al. 1995). 28S D2 regions were sequenced in both directions, while coxI was sequenced only in the forward or both directions. New Synergus haplotypes are deposited in GenBank, under accession numbers KR270530 KR270560 and KR537436 - KR537438 (detailed below.). Further fully overlapping sequences were downloaded from previous studies of gallwasp inquilines (Synergini) (Ács et al. 2010; Pénzes et al. 2009; Melika et al. 2012; Bozsó et al. 2014; Kaartinen et al. 2010, Bernardo et al. 2013, Nylander et al. 2004; detailed below.). CoxI sequences were aligned without difficulty using Muscle 3.8.31 (Edgar et al. 2004) using the default settings as no gaps were detected. 636 sequence positions were involved as characters in the following phylogenetic analysis. Sequences of the 28S D2 region were aligned using MAFFTX-INS-i version 7.157b using the default settings (Katoh et al. 2002, Katoh \& Standley 2013) and resulted in 604 sequence positions. Following the alignments, phylogenetic reconstruction was carried out in a Bayesian framework using MrBayes 3.2.4 64-bit parallel version (Ronquist \& Huelsenbeck 2003, Altekar et al. 2004, Ronquist et al. 2012) for the combined coxI+28S D2 dataset. Separate partitions with varying rate and unlinked parameters were defined for D2 and three coxI codon positions. For all four partitions, four-by-four nucleotide models were selected and a GTR substitution model space were sampled during the MCMC analyses (nst=mixed option) with gamma distributed rate variation across sites. Default priors were used for all parameters.

Two independent runs were performed with the default MCMC parameters except the following settings: MCMC runs comprised 10 million generations sampled every 1000 generations with $30 \%$ considered as burn-in. Sufficient convergence was achieved in the analysis diagnosed by the average standard deviation of split frequencies between the two independent runs $(<0.01)$ and PSRF values ( 1 with $<1 \%$ deviation). Rhoophilus loewi was used to root the phylogenetic tree (Ács et al. 2010). 


\subsubsection{Morphological descriptions}

The terminology used to describe gallwasp morphology follows recent cynipid studies (Melika et al. 2010; Liljeblad et al. 2008). Abbreviations for fore wing venation follow Ronquist \& Nordlander (1989), cuticular surface terminology follows that of Harris (1979). Measurements and abbreviations used here include: F1-F12, 1st and subsequent flagellomeres; POL (postocellar line) is the distance between the inner margins of the posterior ocelli; OOL (ocellarocular line) is the distance from the outer edge of a posterior ocellus to the inner margin of the compound eye; LOL, the distance between lateral and frontal ocelli. The width of the forewing radial cell is measured from the margin of the wing to the Rs vein.

Images of wasp anatomy were produced with a digital Leica DC500 camera attached to a Leica DM2700M compound microscope with using the LAS Store\&Recall software, followed by processing in Adobe Photoshop 6.0. Images of adult wasps were produced by Szabina Schwéger (all Synergus and Saphonecrus species), while some pictures of Ufo, Synophrus and Lithosaphonecrus were taken by Dr. Miklós Bozsó (Plant Health and Molecular Biology Laboratory, National Food Chain Safety Office, Budapest, Hungary). Gall images were taken in the field mainly by Chang-Ti Tang (National Chung Hsing University, Taichung, Taiwan) and

Dr. György Csóka (NARIC, Forest Research Institute, Department of Forest Protection, Mátrafüred, Hungary).

The type material is deposited in the following institutions: PHMB, Plant Health and Molecular Biology Laboratory, National Food Chain Safety Office, Budapest, Hungary (curator G. Melika); NCHU, Department of Entomology, National Chung Hsing University, Taichung, Taiwan (curator C.-T. Tang); USNM, U.S. National Museum of Natural History, Smithsonian Institution, Washington, DC, U.S.A. (curator M. Buffington). Morphological peculiarities are given in Appendix 9.1.

\subsection{Oak gallwasps (Cynipidae: Cynipini)}

The list of EP Cynipini includes those oak gallwasps (Cynipidae: Cynipini) whose names are (i) valid according to the rules of the International Code of Zoological Nomenclature (1999) (ICZN) and (ii) which were regarded as real biological entities in the literature. The vast majority of the listed species have been collected many times by G. Melika and other co-authors in Schwéger et al. (2015a, b) in different parts of the EP (Far East of Russia, Japan, South Korea, 
China, Taiwan) and research done on those species (taxonomic and/or molecular phylogenetic and/or rearing work) supports their existence as distinct biological units. Rare species listed have also been carefully checked (type material of adult wasps and galls was examined where possible by G. Melika, and the relevant literature was analysed by me). This work is made more difficult by the fact that the types of some species have been lost (for example, some species described by Shinji (1938, 1940, 1941) from Japan). Recent nomenclatorial changes have been taken into account and these mean that some generic names are no longer used. All known literature names for specific species are linked to the currently recognised names under the Synonyms part. There are EP oak gallwasp species names in the literature that, although valid in the strict taxonomic sense, represent uncertain or questionable taxa. Some appeared in the literature only in the original description and have never since been collected or cited. Such species (species names) are also listed as nomina dubia (species with uncertain status) and are given after the valid species list.

Summaries are presented for each species in alphabetical order in Appendix 9.2. in the following format:

Synonyms. All known synonyms are given and, where appropriate, they are attributed to specific generations in the lifecycle. The names of many species have changed since their original description and thus we are listing all known synonyms for each species and generation. The validity of a taxon name according to the International Code of Zoological Nomenclature (ICZN) is based on the priority principle: valid species name is that which was first published. All other later published names that refer to the same species are junior synonyms. Gallwasp nomenclature is unusual in comparison to other insect groups in that in many cases alternate generations of a single biological entity were described as different species or even as members of different genera, and only later found to represent halves of the same lifecycle. In this case, the name of the first generation to be described is the valid name for the species, the second generation's name becoming a junior synonym. So for oak gallwasps the term "synonym" refers both to real synonyms of a known 2-generation species and to synonyms linking alternate generations. We have included not only synonyms, but also all existing name combinations as the generic arrangement of many species has evolved over time.

Lifecycle. We say which of sexual and asexual generations are known for the species, together with any specific literature involved in linking generations. 
Geographic distribution. We provide an overview of the updated distribution of each species, and list the countries from which each species has been recorded across the EP.

Host plants. The names used follow the revision by Govaerts \& Frodin (1998).

Gall location and structure. The plant part affected by a given gall is described. We describe the structure of the mature gall and, where possible, also summarise changes that take place during gall development. Galls are described as being unilocular (containing a single larval chamber) and multilocular (a single structure containing many larval chambers). Unilocular galls may be solitary or gregarious (many discrete galls clustered together). Some unilocular galls are also able to coalesce into an apparently multilocular structure when they develop in close proximity.

Phenology. Data on the timing of gall development, the frequency and duration of diapause and of the flight periods of the adults are provided.

Similar galls. We describe galls induced by other species that could be confused with the gall in question, and summarise diagnostic characters for their identification.

In Appendix 9.3. the list of EP Cynipini species with uncertain status (nomena dubia or incertae sedis) is given. A common way for a taxon name to become a nomen dubium is loss of the original type material, which prevents revision of the taxon and establishment of synonymy with other taxa. 


\section{RESULTS}

\subsection{Synergini sensu stricto}

Besides the tribe Ceroptresini, the tribe Synergini sensu stricto comprises inqulines associated mainly with Fagaceae. Table 2 includes currently known 7 genera belong to this tribe with the number of all the newly (Synergus and Saphonecrus) and recently (Synophrus, Ufo, Lithosaphonecrus) described species (Pénzes et al. 2009, Bozsó 2015, Bozsó et al. 2014, 2015, Melika et al. 2012, Schwéger et al. 2015a, b). To keep the integrity of formal descriptions, general comments are given in the Results section, too.

\subsubsection{Key to Cynipidae tribes}

For the identification of the Cynipidae tribes the next key is proposed, with original colour plates (unpublished). The key follows that of Ronquist et al. (2015), with some modifications and simplifications.

\section{Key to Cynipidae tribes}

1. Female antenna clavate; last flagellomere wider than penultimate; male antenna with either F2, F3 or both modified; ventral area of gena with 5-9 vertical carinae; dorsolateral margin of pronotal plate strongly projecting laterad; scutellar foveae shallow or indistinct; mesopleural impression present, conspicuous

Paraulacini

- Female antenna filiform or slightly expanded towards apex; last flagellomere not wider than penultimate; male antenna not modified or with only F1 modified; ventral area of gena without vertical carinae; dorsolateral margin of pronotal plate not projecting laterad; scutellar foveae and mesopleural impression present or absent

2. Frons between antennal toruli with strong longitudinal carina; notaulus and scutellar foveae absent; dorso-axillar area large, triangular and situated in same plane as mesoscutellum; Rs+M and R1 of fore wing inconspicuous, basal vein absent

Eschatocerini

- Frons between antennal toruli without strong longitudinal carina; notaulus and scutellar foveae usually present; dorso-axillar area situated in different plane compared to mesoscutellum; Rs+M and R1 of fore wing usually conspicuous, basal vein present 3

3. Scutellar foveae faint or absent (Figs 1, 4); mesopleuron with longitudinal impression (Figs 2, 5); female antenna with 12 or more flagellomeres; male F1 not modified; hypopygium ploughshaped (Figs 3, 7) or hypopygial spine short (Fig. 6) 
- Scutellar foveae usually well differentiated, deep, sometimes confluent and forming transverse depression (Figs 8, 11, 14); mesopleuron smooth or sculptured, without longitudinal impression (Figs 9, 12, 15); female antenna usually with 10-11 flagellomeres, rarely more; male F1 usually modified; hypopygium never plough-shaped, with short or long hypopygial spine (Figs 10, 13, 16) .5

4. Pronotum short dorsomedially, admedian depressions of pronotum not visible, pronotal plate absent; mesopleural impression broad, crenulate (Fig. 2); mesoscutellum dorsally convex, without rounded impressed area (Fig. 1); lateral propodeal carinae absent (Fig. 17); hypopygium plough-shaped (Fig. 3)

Diplolepidini

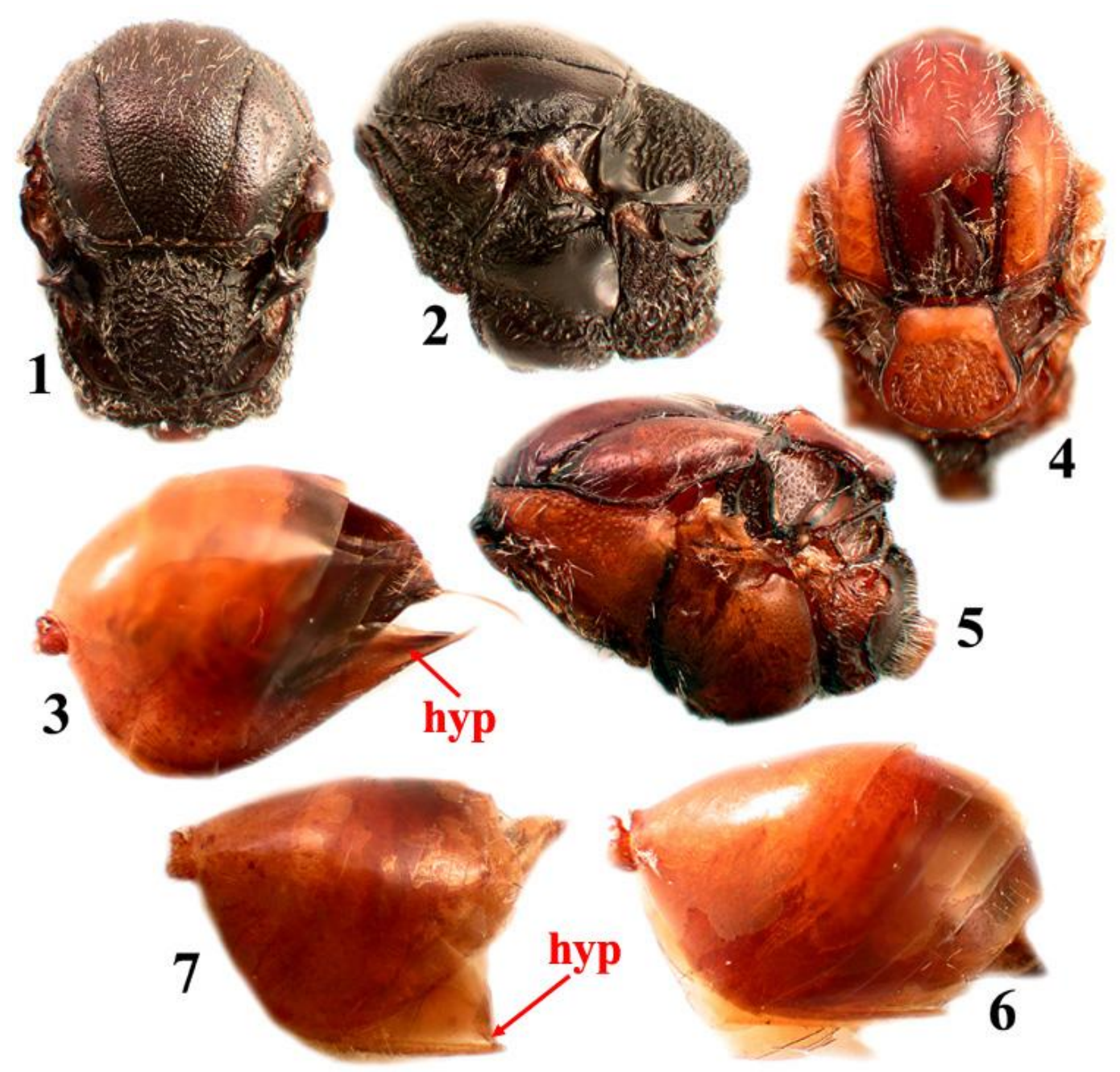

FIGURES 1-7. 1-3, Diplolepis rosae, female: 1, mesosoma, dorsal view, 2, mesosoma, lateral view, 3, metasoma, lateral view (hyp, hypopygium). 4-6, Pediaspis aceris, asexual female: 4, mesosoma, dorsal view, 5, mesosoma, lateral view, 6, metasoma, lateral view. 7, Pediaspis aceris, sexual female, metasoma, lateral view (hyp, hypopygium). 
- Pronotum longer dorsomedially, admedian depressions of pronotum clearly visible and with conspicuous pronotal plate; mesopleural impression linear, narrow, without crenulate sculpture (Fig. 5); mesoscutellum dorsally flat with rounded impressed area (Fig. 4); lateral propodeal carinae present (Figs 18-19); hypopygium not plough-shaped (Figs 6-7) Pediaspidini

5. Occiput with strong, sharp occipital carina (Fig. 20); hypopygium abrupt, not prolonged into a ventral spine; with dense tuft of long setae (Fig. 16); radial cell closed on anterior margin of fore wing

Qwaqwaiini

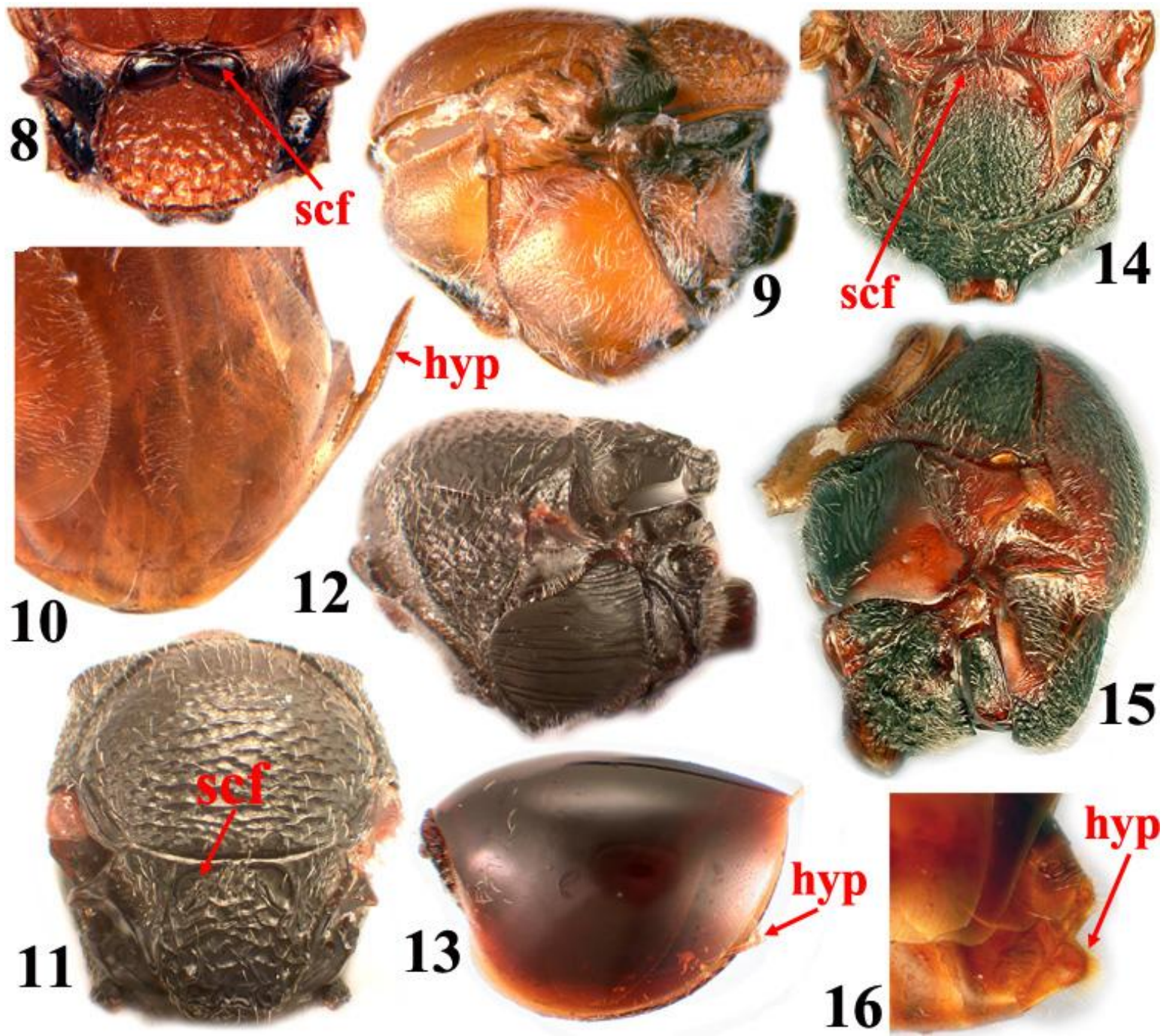

FIGURES 8-16. 8-10, Andricus anatolicus, asexual female: 8, mesoscutellum, dorsal view (scf, scutellar fovea), 9, mesosoma, lateral view, 10 metasoma, part, lateral view (hyp, hypopygium). 11-13, Saphonecrus undulatus, female: 11, mesosoma, dorsal view (scf, scutellar fovea), 12, mesosoma, lateral view, 13, metasoma, part, lateral view. 14-16, Qwaqwaia scolopiae, female: 14, mesoscutellum, dorsal view (scf, scutellar fovea), 15, mesosoma, lateral view, 16, metasoma, part, lateral view (hyp, hypopygium). 
- Occiput without distinct, sharp occipital carina (Figs 21-22); hypopygium with more or less distinct, elongated, needle-like ventral spine, with subapical setae only rarely forming dense tuft (Figs 10, 13); radial cell closed or open
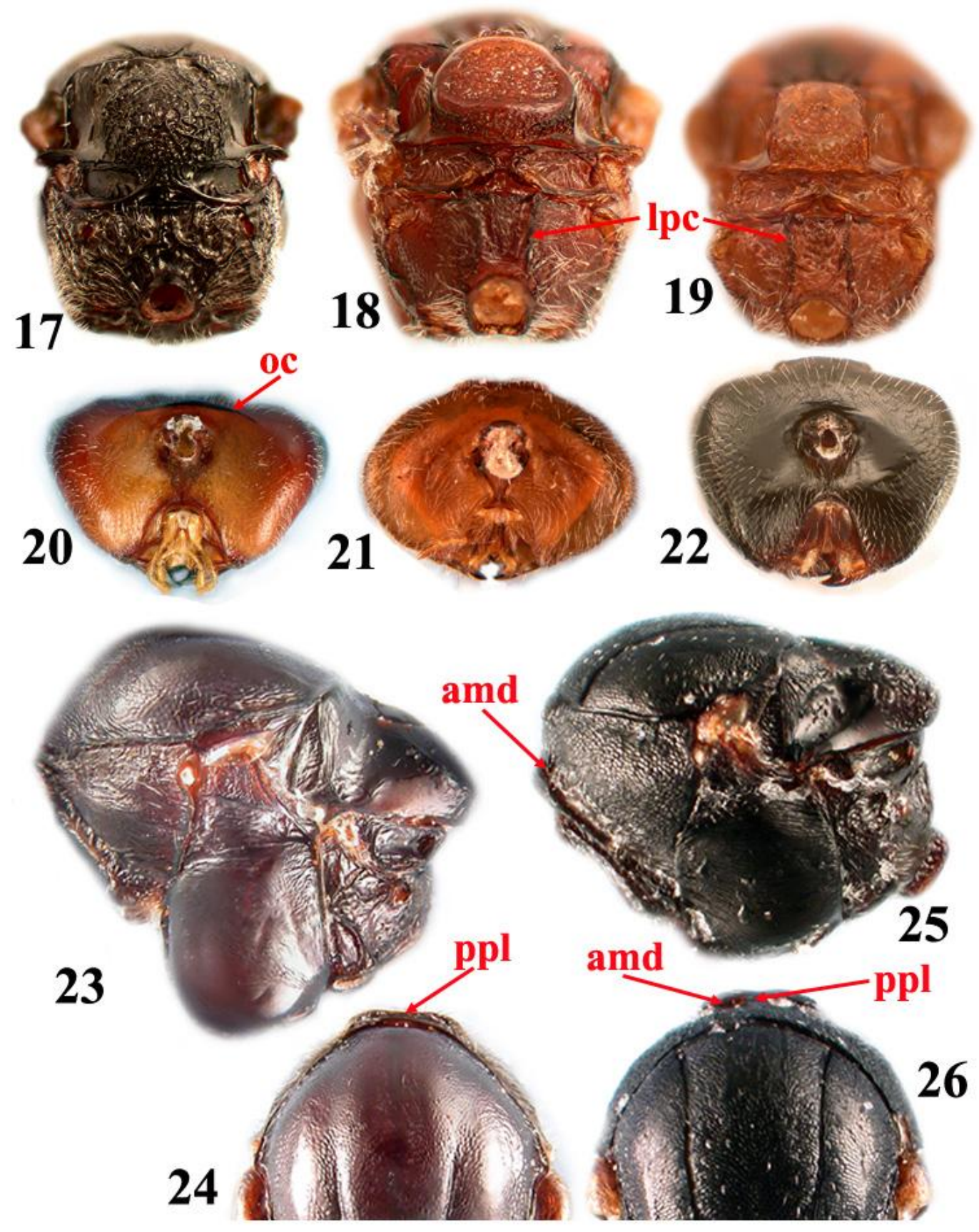

FIGURES 17-26. 17-19, propodeum, posterodorsal view: 17, Diplolepis rosae, female, 18, Pediaspis aceris, asexual female (lpc, lateral propodeal carina), 19, Pediaspis aceris, sexual female. 20-22, head, posterior view: 20, Qwaqwaia scolopiae, female (oc, occipital carina), 21, Andricus anatolicus, asexual female, 22, Saphonecrus undulatus, female. 23-24, Neuroterus quercusbaccarum, asexual female: 23, mesosoma, lateral view, 24, mesoscutum, part, dorsal view (ppl, pronotal plate). 25-26, Aulacidea acroptilonica, female: 25, mesosoma, lateral view (amd, admedian depression), 26, mesoscutum, part, dorsal view (amd, admedian depression, ppl, pronotal plate). 
6. Basal part of pronotal plate small, short medially; admedian depressions of pronotum absent, or forming weak, shallow, continuous depression; pronotum dorsomedially short, 1/7 or less of length of outer lateral margin (Figs 23-24)

Cynipini

- Basal part of pronotal plate usually bigger, visible; admedian depressions of pronotum usually clearly visible, more or less widely separated (Fig. 26); pronotum dorsomedially longer, 1/5 to $1 / 3$ as long as greatest length of outer lateral margin, rarely shorter but then admedian depressions present (Figs 25-26)

7. Distinct raised vertical carina from ventral margin of antennal socket present, at least close to antennal socket (Fig. 27); 2nd metasomal tergite in both sexes small, free, not fused with fourth, with dense hair patch anterolaterally (Fig. 28); female antenna with 10 flagellomeres; radial cell of fore wing always closed along anterior margin

Ceroptresini

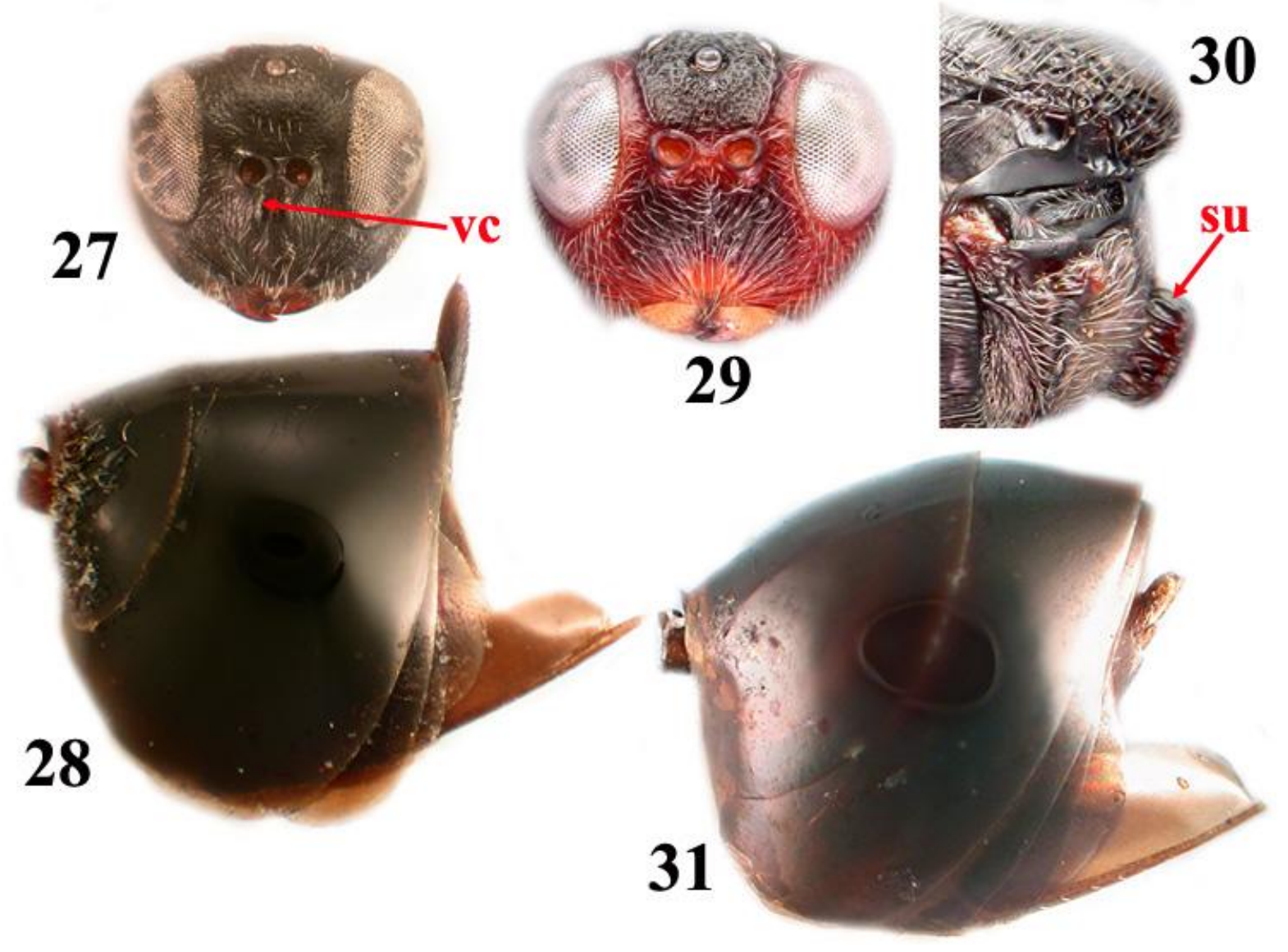

FIGURES 27-31. 27-28, Ceroptres cerri, female: 27, head, frontal view (vc, vertical carina), 28, metasoma, lateral view. 29, Synergus formosanus, female, head, frontal view; 30, mesosoma, posterior part, lateral view (su, longitudinal sulci on nucha), 31, Aulacidea acroptilonica, female, metasoma, lateral view. 
- Distinct raised vertical carina from ventral margin of antennal socket absent, sometimes several weaker carinae present in this region (Fig. 29); 2nd metasomal tergite either free, but not small, or fused with fourth into one large syntergite (Figs 13, 31); anterolateral hair patch present or not; female antenna with 10-12 flagellomeres; radial cell of fore wing open or closed along anterior margin 8

8. Abdominal terga $3+4$ in both sexes fused into one large syntergite, occupying nearly entire metasoma (Fig. 13); dorsal part of pronotal plate incomplete, its lateral margins marked only ventrally (Figs 11-12); head and/or mesosoma usually strongly sculptured (Fig. 29); nucha and first ring-shaped metasomal tergite with longitudinal sulci (Fig. 30)

Synergini sensu stricto

- Abdominal terga 3-8 free in most cases (Fig. 31); if terga 3+4 fused in females into one large sclerite then the corresponding terga not fused in males and pronotal plate distinct, with lateral margins marked almost entirely (Fig. 26); nucha without longitudinal sulci (Fig. 25); first metasomal tergite usually crescent-shaped and smooth (Fig. 31) Aulacini sensu lato*

*includes Diastrophini (with Periclistus and Synophromorpha), Phanacidini, Aylacini sensu stricto, Aulacideini.

\subsubsection{Key to Synergini sensu stricto genera}

For the identification of the seven inquiline genera of Synergini, we propose the following key, which is the most updated one, including elements and new pictures which were not presented in other, earlier proposed Synergini keys (Pénzes et al. 2012).

\section{Key to Synergini sensu stricto genera}

1. First metasomal tergite smooth, reduced to dorsal crescent-shaped projecting scale (Fig. 32); clypeus distinctly separated from lower face, anterior tentorial pit deep (Fig. 33) ..... Rhoophilus - First metasomal tergite in a form of ring or collar, sulcate at least laterally (Fig. 34); clypeus indistinctly separated from lower face, anterior tentorial pit indistinct (Fig. 35) ....................... 2 2. Head trapezoid in anterior view, strongly convex ventrally (Fig. 36); 2.5-2.8x as broad as long in dorsal view; interocellar triangle narrow, posterior edge of frontal ocellus lies on a line between anterior edges of lateral ocelli (Fig. 37); anterior part of pronotum rectangular in dorsal 
view, right angle between anterior and lateral sides present; lateral part of pronotum going down from dorsal part also nearly at a right angle; strong pronotal carina divides lateral part from frontal, both of which oriented almost at a right angle to each other; dorsomedian part of pronotum invisible in dorsal view (Figs 38-39) Ufo
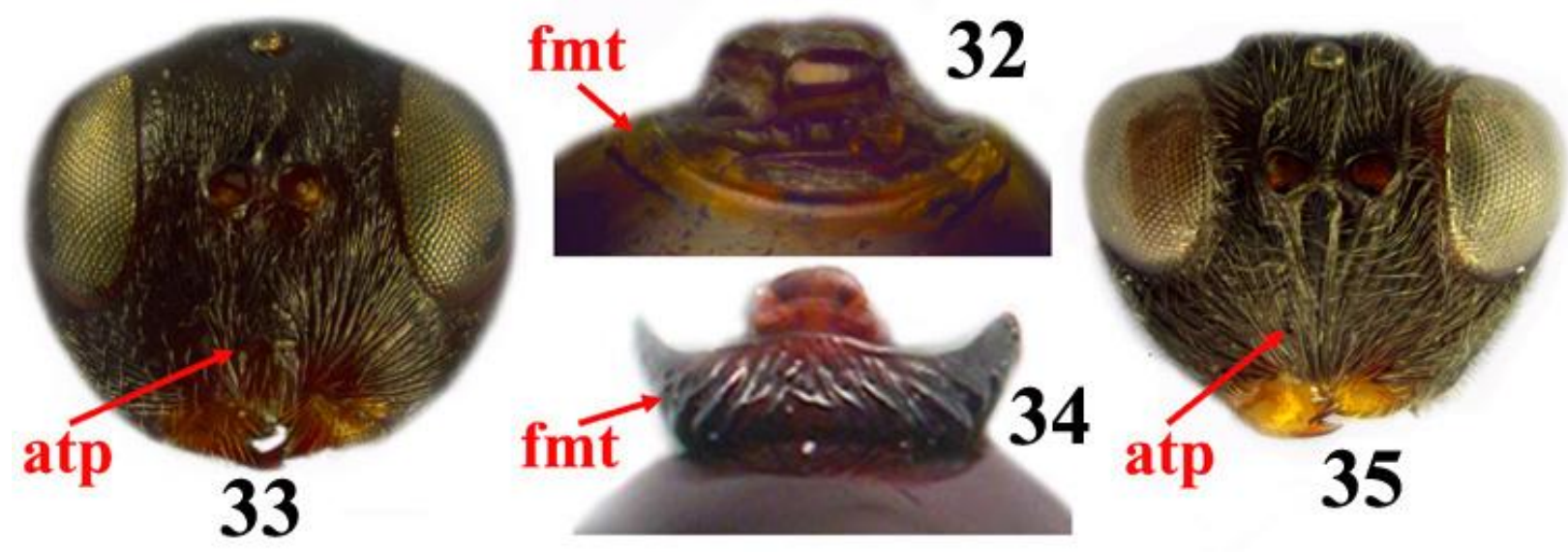

FIGURES 32-35. 32-33, Rhoophilus loewi, female: 32, first metasomal tergite (fmt), dorsal view, 33, head, frontal view (atp, frontal tentorial pit). 34, Synergus kawakamii, female, first metasomal tergite (fmt), dorsal view. 35, Synergus belizinellus, female, head, frontal view (atp, frontal tentorial pit).

- Head rounded or ovate in anterior view, less convex ventrally (Figs 40, 44, 48, 50); 1.6-2.1x as broad as long from dorsal view; interocellar triangle always broader, posterior edge of frontal ocellus lies away from a line between anterior edges of lateral ocelli (Figs 41, 45, 49, 51); lateral pronotal carina absent or present; right angle between anterior and lateral sides absent; dorsomedian part of pronotum visible in dorsal view (Figs 42-43, 46-47, 52-53)

3. Pedicel 2.5x as long as scape and F2; male antenna with 11 flagellomeres Agastoroxenia - Pedicel shorter than scape and F2; male antenna with 12-14 flagellomeres 4 4. Lateral frontal carina present (Fig. 44); radial cell of forewing usually closed (Fig. 57), if open or partially open (in Synergus kawakamii and S. castaneus), then lateral pronotal carina present Synergus

- Lateral frontal carina absent (Figs 48, 50); radial cell of forewing open (Fig. 58) 5

5. Metapleural sulcus reaching posterior margin of mesopectus slightly higher than half of its height (Fig. 53); first metasomal tergite smooth medially, sulcate only laterally (Figs 54-55) 


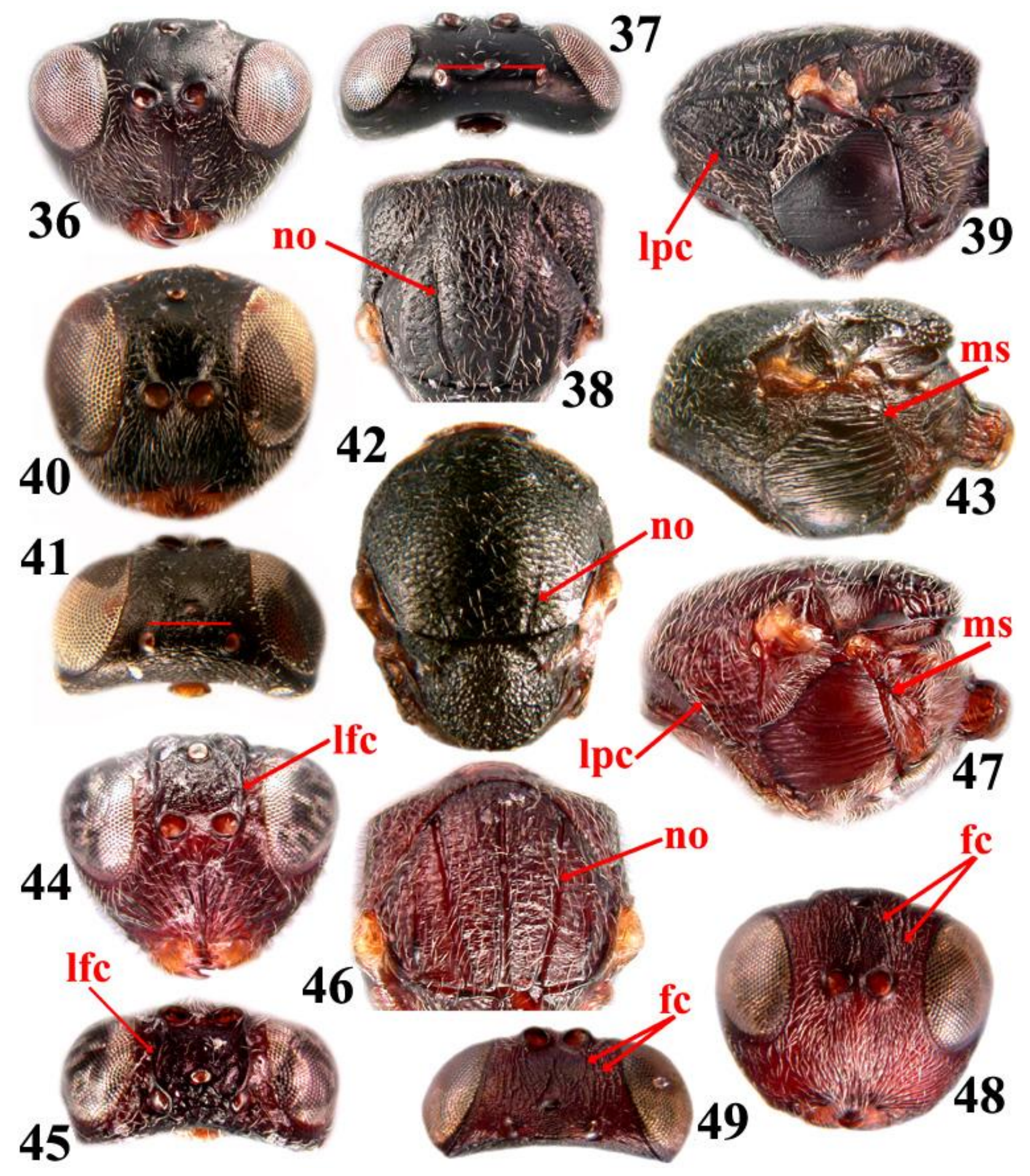

FIGURES 36-49. 36-39, Ufo nipponicus, female: 35-37, head: 36, frontal view, 37, dorsal view (red line indicates that laterall ocelli in one row with frontal ocellus); 38-39, mesoscutum: 38, dorsal view (no, notaulus), 39, lateral view (lpc, lateral propodeal carina). 40-43, Saphonecrus connatus, female: 40-41, head: 40, frontal view, 41, dorsal view; 42-43, mesoscutum: 42, dorsal view, 43, lateral view (ms, metapleural sulcus). 44-47, Synergus xialongmeni, female: 44-45, head: 44, frontal view (lfc, lateral frontal carina), 45, dorsal view; 46-47, mesoscutum: 46, dorsal view, 47, lateral view. 48-49, Lithosaphonecrus huisuni, female, head: 48, frontal view, 49, dorsal view (fc, frontal carinae). 

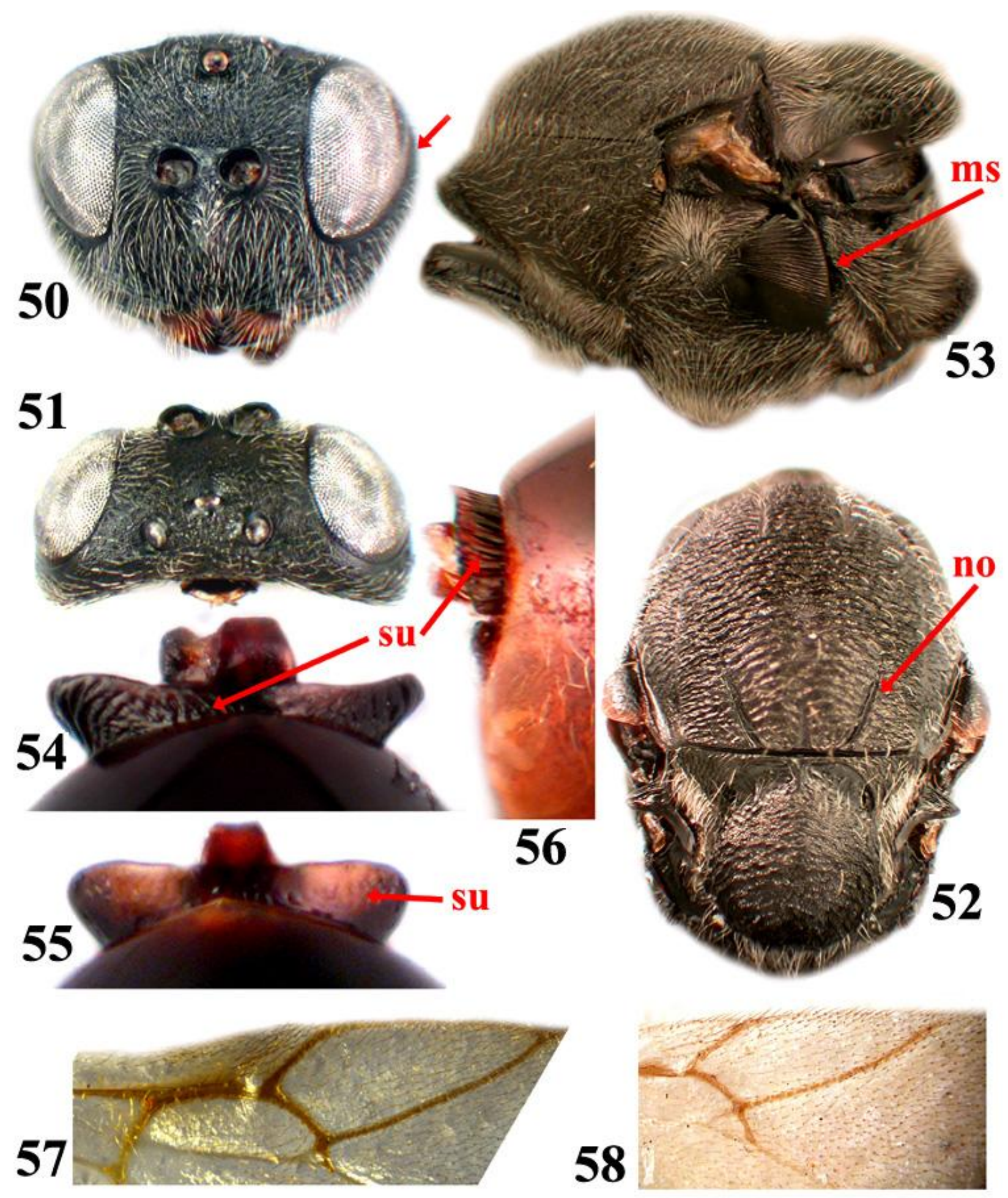

FIGURES 50-58. 50-51, Synophrus hungaricus, female, head: 50, frontal view (red arrow indicates broadened gena behind eye), 51, dorsal view. 52-54, Synophrus politus, female: 52-53, mesoscutum: 52, dorsal view, 53, lateral view (ms, metapleural sulcus); 54, first metasomal tergite, dorsal view (su, longitudinal sulci). 55, Synophrus pilulae, female, first metasomal tergite, dorsal view. 56, Synergus umbraculus, female, first metasomal tergite, lateral view. 57-58, forewing, part, female: 57, Synergus khazani, 58, Saphonecrus chaodongzhui. 
- Metapleural sulcus reaching posterior margin of mesopectus at 1/4-1/5 of its height (Fig. 43); first metasomal tergite sulcate laterally and dorsally (Figs 56, 59-60)

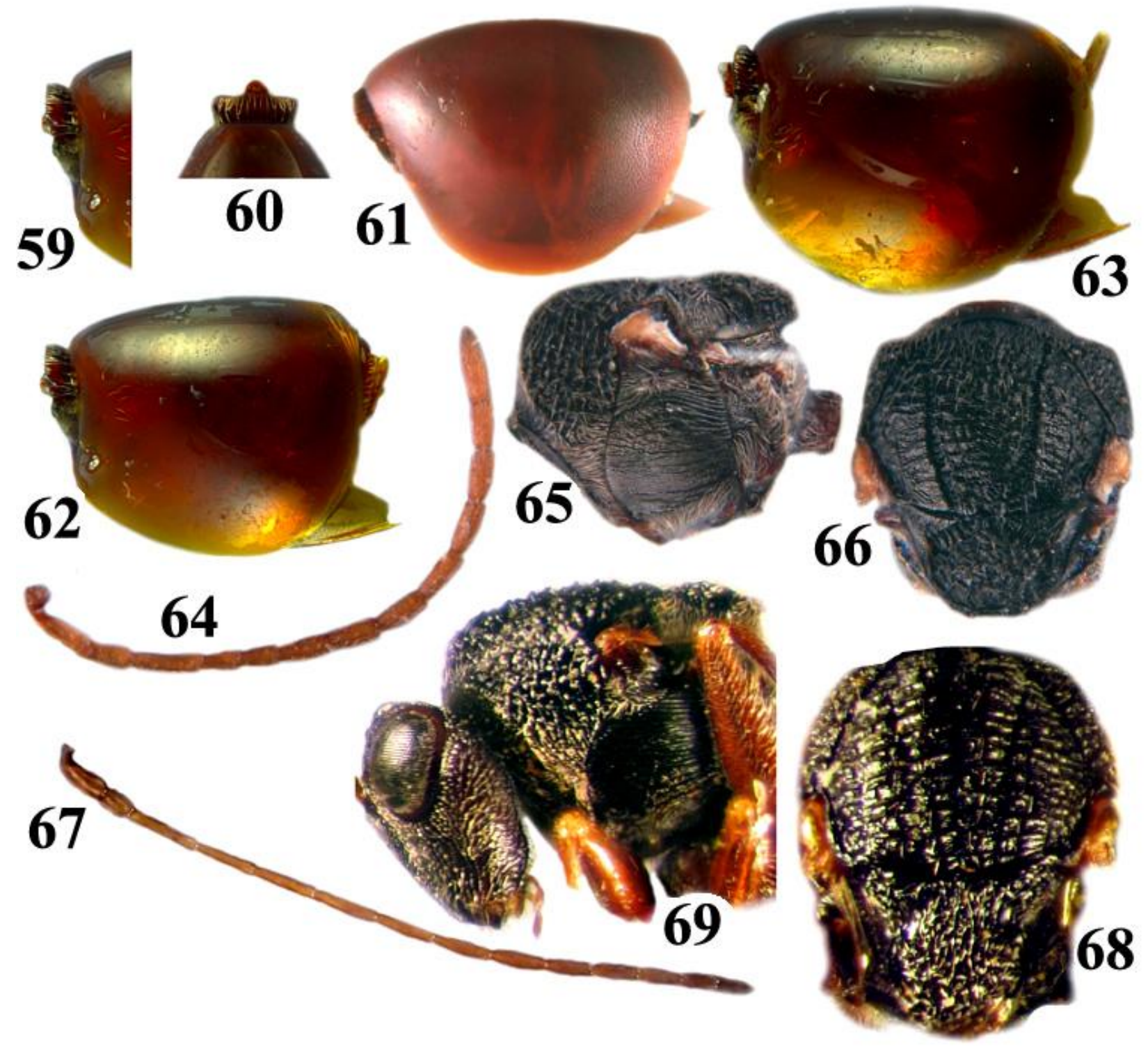

FIGURES 59-69. 59-60, Saphonecrus chinensis, metasoma: 59, part, lateral view, 60, part, dorsal view. 61-63, metasoma, female, lateral view: 61, Lithosaphonecrus huisuni, 62, Saphonecrus chinensis, 63, Saphonecrus robustus. 64-66, Lithosaphonecrus dakengi, female: 64, antenna, 65-66, mesosoma: 65, dorsal view, 66, lateral view. 67-69, Saphonecrus serratus, female: 67, antenna, 68-69, mesosoma: 68, dorsal view, 69, lateral view.

6. Frons with numerous parallel rugae, extending from toruli to lateral ocelli (Figs 48-49); syntergite posteriorly punctured or reticulate, with sculptured band extending for at least to 1/4-1/5 length of syntergite and reaches ventral edge of tergite (Fig. 61) ..................................... 7 
- Frons without frontal rugae, smooth, alutaceous, microreticulate, micropunctate or coriaceous (Figs 50-51); syntergite neither punctured nor reticulate, if indistinct punctures present than only as dorsoposterior patch, punctures never reaches ventral edge of tergite (Figs 62-63)

Saphonecrus

7. Female antenna with 11 flagellomeres, female F1 1.5-1.9x as long as F2 (Fig. 64); lateral pronotal carina complete, sides of pronotum sharply angled in dorsal view (Figs 65-66); body length $1.4-1.9 \mathrm{~mm}$ Lithosaphonecrus

- Female antenna with 12 flagellomeres, female F1 1.2x as long as F2 (Fig. 67); lateral pronotal carina partially present, weak, sides of pronotum rounded in dorsal view (Figs 68-69); body length $2.7 \mathrm{~mm}$ Saphonecrus serratus*

*for easier identification and some unique characters, S. serratus was keyed out away from Saphonecrus.

Below a brief overview for the Synergini sensu stricto genera is given in alphabetical order (Pénzes et al. 2012, Schwéger et al. 2015a, b).

\subsubsection{Agastoroxenia Nieves-Aldrey \& Medianero, 2010}

Morphologically, Agastoroxenia is related to Saphonecrus and Synophrus by the 13-segmented antenna in females; however, in males the antenna is also 13-segmented which is a unique autapomorphic feature among all the known genera of Synergini. With slightly expanded genae, weakly sulcated dorsal part of 1st metasomal tergite and the general sculpture of the mesoscutum and mesopleuron, this genus resembles Synophrus, but it has strong frontal carinae, a character state that is shared by the majority of the Synergus species. This genus, with one known species, Agastoroxenia panamensis Nieves-Aldrey \& Medianero, is distributed in Panama, an inquiline reared from an unidentified Andricus stem gall on Q. lancifolia (Quercus, section Lobatae) (Nieves-Aldrey \& Medianero 2010). We were unable to obtain specimens for DNA isolation, thus this genus is not represented in our phylogenetic analysis.

\subsubsection{Lithosaphonecrus Tang, Melika \& Bozsó, 2013}

Only five EP Saphonecrus species, S. shirakashii, S. shirokashicola, S. naiquanlini, S. yukawai, and S. excisus, share the following two characters with Lithosaphonecrus: the female antenna 
with 11 flagellomeres and presence of a lateral pronotal carina. However, morphologically Lithosaphonecrus is a very distinct genus containing species exclusively associated with Lithocarpus species. A number of unique morphological characters separate the genus from all other inquiline species are as follows: rounded or very slightly trapezoid robust head with irregular interrupted frontal carinae extending from toruli to ocelli; the mesopleuron is reticuloso-striate; scutellar foveae are confluent or if median carina present then with an indistinct, glossy base with strong longitudinal parallel wrinkles; fused metasomal tergites $2+3$ are posteriorly punctured or reticulate, the sculptured band extending for at least to $1 / 4-1 / 5$ length of fused tergites, always reaching the ventral edge of the tergite. Moreover, in Lithosaphonecrus species, F1 in the female antenna 1.5-1.9x longer than F2, F11 2.0x longer than F10; F1 in male antenna 2.6-3.0x longer than F2, while in the above-mentioned Saphonecrus species, F1 in the female and male antennae are 1.1-1.4x longer than F2; F11 in the female antenna always nearly equal to F10 length. In the mentioned Saphonecrus species, the frons is always smooth or alutaceous, shiny, never with carinae as in Lithosaphonecrus and metasomal tergite $2+3$ without punctured or reticulate posterior band; if indistinct punctures present then only in a form of an anteroposterior patch, micropunctures never reach the ventral edge of the tergite. Molecular phylogeny support that Lithosaphonecrus forms a distinct lineage within Synergini, was described from China and Taiwan (Bozsó et al. 2015, online version in 2013). Lithonecrus Nieves-Aldrey \& Butterill, with one species (L. papuanus Nieves-Aldrey \& Butterill), emerged from undescribed galls, collected from Lithocarpus celebicus (Miq.) Rehd., was described from Papua New Guinea (Nieves-Aldrey \& Butterill 2014). The only diagnostic character that was given by the authors to distinguish Lithonecrus from Lithosaphonecrus is the presence of the lateral pronotal carina in Lithonecrus and its absence in Lithosaphonecrus. However, the lateral pronotal carina is present in all Lithosaphonecrus species, too (Bozsó et al. 2015, online 2013). All other characters are the same and thus, Lithonecrus was synonymized to Lithosaphonecrus (Schwéger et al. 2015b). Unfortunately no "Lithonecrus" specimens were obtained for molecular phylogenetic analysis. Thus, Lithosaphonecrus papuanus (Nieves-Aldrey \& Butterill, 2014) has been established. Recently, a new inquiline, Synophrus vietnamensis Abe, Ide, Konishi \& Ueno was described from Vietnam (Abe et al. 2014a). The examination of the detailed description and provided illustrations showed that this species errouneosly was assigned to Synophrus and based on all characters, it was transferred to Lithosaphonecrus: Lithosaphonecrus 
vietnamensis (Abe, Ide, Konishi \& Ueno) (Schwéger et al. 2015b). A key to species was given in Bozsó et al. (2015). Currently known Lithosaphonecrus species are summarized in Table 4. Morphological peculiarities and host galls of Lithosaphonecrus are depicted in Appendix 9.1. (Figs A1-A24).

Table 4. Lithosaphonecrus species: distribution and host associations

\begin{tabular}{|l|c|c|c|}
\hline \multicolumn{1}{|c|}{ Lithosaphonecrus species } & Distribution & Host gallwasp & Host plant \\
\hline L. formosanus Melika \& Tang, 2013 & Taiwan & $\begin{array}{c}\text { undescribed bud, } \\
\text { catkin and stem galls }\end{array}$ & $\begin{array}{c}\text { Lithocarpus konishii } \\
\text { L. } \text { hancei } \\
\text { and L. glabra }\end{array}$ \\
\hline L. dakengi Tang \& Pujade-Villar, 2013 & Taiwan & bud gall & L. hancei \\
\hline L. huisuni Tang, Bozsó \& Melika, 2013 & Taiwan & bud gall & L. glabra \\
\hline L. yunnani Tang, Bozsó \& Melika, 2013 & China & bud gall & L. fenestratus \\
\hline $\begin{array}{l}\text { L. vietnamensis } \\
\text { Abe, Ide, Konishi \& Ueno, 2014 }\end{array}$ & Vietnam & bud gall & Castanopsis sp. \\
\hline $\begin{array}{l}\text { L. papuanus } \\
\text { Nieves-Aldrey \& Butterill, 2014 }\end{array}$ & $\begin{array}{c}\text { Papua } \\
\text { New Guinea }\end{array}$ & unknown gall & L. celebicus \\
\hline
\end{tabular}

\subsubsection{Rhoophilus Mayr, 1881}

Rhoophilus is morphologically related to the Holarctic inquiline genera Synergus, Saphonecrus, and Synophrus, all of which typically attack oak cynipid galls (van Noort et al. 2007). A sister group relationship between Rhoophilus and the oak inquiline genera Synergus + Synophrus + Saphonecrus was hypothesized by Ronquist (1994) and Liljeblad \& Ronquist (1998). Shared diagnostic characters include the following: the ventral margin of the clypeus is straight, not projecting over mandibles; radiating striae on the lower face reaching or almost reaching the compound eye; the distance between occipital and oral foramina is longer than the height of the occipital foramen; the position of the anterior end of the metapleural sulcus is high; the mesoscutum with strong transverse ridges, the mesopleuron also with longitudinal ridges; tarsal claws with a blunt small basal lobe. Rhoophilus loewi and the recently described south african cynipid gall-inducer Qwaqwaia scolopiae Liljeblad, Nieves-Aldrey \& Melika on Scolopia mundii (Salicaceae) represent the only cynipid taxa with an Afrotropical (Aethiopian) distribution (Liljeblad et al. 2011). Eschatocerus (gall inducers on Acacia and Prosopis) and Rhoophilus may represent the remnants of the oldest primitive lineages of cynipids (Nylander et al. 2004, Ronquist et al. 2015). Morphological peculiarities of Rhoophilus are depicted in Appendix 9.1. (Figs A25-A39). 


\subsubsection{Synophrus Hartig, 1843}

Morphologically, Synophrus appears most closely related to Saphonecrus (Melika 2006, Pénzes et al. 2009). Two morphological characters have been suggested to separate Synophrus from Saphonecrus: in Synophrus the metapleural sulcus reaches the anterior margin of the metapleuron at half or slightly higher of its height and the 2nd metasomal tergite has longitudinal sulci only laterally, being smooth dorsally, while in Saphonecrus the metapleural sulcus reaches the anterior margin of the metapleuron in the upper $1 / 3$ of its height, and the entire 2 nd metasomal tergite has longitudinal sulci (Pujade-Villar et al. 2003). In Synophrus lateral frontal carinae are absent; a male antenna has 13 flagellomeres; lateral propodeal carina is absent, the pronotum is rounded in dorsal view; the radial cell in the forewing is opened (Pénzes et al. 2009). Currently 7 Synophrus species are known, all from the Western Palearctic only (Table 5) and all of which are able to impose their own gall phenotypes on those of their hosts (Pénzes et al. 2009). Originally described as a gall inducer (Hartig 1843), Synophrus was later transferred to the Synergini on the basis of adult morphology (Ronquist 1994). An inquiline life history is supported by further evidence. It was observed that $S$. politus emerged from irregularly spherical and highly lignified stem swelling galls that developed over the summer in the exact location in which spring bud galls of a known gall inducing wasp, Andricus burgundus Giraud were initiated (Pujade-Villar et al. 2003). This modification of the host gall is extreme among cynipid inquilines, and attack by Synophrus is always lethal to the host gallwasp. Synophrus is known from section Cerris of genus Quercus exclusively. Morphological peculiarities and modified galls of Synophrus are depicted in Appendix 9.1. (Figs A40-A48).

Table 5. Synophrus species: distribution and host associations

\begin{tabular}{|l|l|l|}
\hline Synophrus species & \multicolumn{1}{|c|}{ Distribution } & \multicolumn{1}{|c|}{ Host plants/galls } \\
\hline S. hungaricus Melika \& Mikó, 2009 & WP: Hungary & Quercus sect. Cerris \\
\hline S. libani Melika \& Pujade-Villar, 2009 & WP: Lebanon & Quercus sect. Cerris \\
\hline S. olivieri Kieffer, 1898 & WP: N.Africa, Iran, Caucasus & Quercus sect. Cerris \\
\hline S. pilulae Houard, 1911 & WP: Austria, Hungary & Quercus sect. Cerris \\
\hline S. politus Hartig, 1843 & WP: Europe, Turkey, Jordan & Quercus sect. Cerris \\
\hline S. syriacus Melika, 2009 & WP: Iran, Syria & Quercus sect. Cerris \\
\hline S. hispanicus Pujade-Villar, 2009 & WP: Iberia & Quercus sect. Cerris \\
\hline Total: 7 species & & \\
\hline
\end{tabular}




\subsubsection{Ufo Melika \& Pujade, 2005}

Ufo was described from Japan with one species, U. abei Melika \& Pujade-Villar (Melika et al. 2005). Later, $U$. koreanus Melika, Pujade-Villar \& Choi was described from Republic of Korea (Melika et al. 2007). Both species are inquilines in oak galls on Quercus subgenus Quercus section Cerris (Fagaceae). All Ufo species are known only from EP, synapomomorphies and generic diagnostic characters of which were discussed in details earlier (Melika et al. 2005, 2007). Ufo shares some morphological characters with two allied genera, Saphonecrus and Synergus. Ufo and Saphonecrus, have the radial cell along the forewing margin opened and the female antenna is 13-segmented; both Ufo and Synergus have a distinct pronotal carina but in Synergus the forewing is with a closed radial cell and the female antenna is 14-segmented (Melika et al. 2005). These shared morphological characters place Ufo into the Synergini sensu stricto. Comparing to Synergus and Saphonecrus, Ufo possesses with a few synapomorphies: the head is trapezoid in frontal view and very narrow in dorsal view, rectangular aspect of the pronotum has distinct rectangular aspect in dorsal view, the tarsal claw with a very acute basal lobe. Based on these characters $U f o$ forms a distinct group within Synergini. Two species, $U$. shirakashii (Shinji) and U. shirokashicola (Shinji), were described from Japan from cynipid galls associated with Quercus subgenus Cyclobalanopsis (Wachi et al. 2011b) and later were transferred to Saphonecrus (Melika et al. 2012). Currently 4 Ufo species are known from the EP: U. abei Melika \& Pujade-Villar, 2005, U. koreanus Melika, Pujade-Villar \& Choi, 2007, U. cerroneuroteri Tang \& Melika, 2012, U. nipponicus Melika, 2012. All these species are known to associate with oak gallwasps developing in section Cerris of genus Quercus oaks (Melika et al. 2012). Molecular phylogeny suggests that it is a sister taxon of a recently described Saphonecrus lineage (Pénzes et al. 2012, Bozsó et al. 2014, Schwéger et al. 2015b). Morphological peculiarities and host galls of Ufo are depicted in Appendix 9.1. (Figs A49-A70).

\subsection{Saphonecrus Dalla Torre, 1910}

\subsubsection{General comments}

Saphonecrus closely resembles Synergus (Pénzes et al. 2009, Ács et al. 2010, Melika et al. 2012, Schwéger et al. 2015a, b). The two genera can be separated by a combination of characters: 
Saphonecrus species have an open radial cell in the fore wing, the female antenna usually with 11 flagellomeres, and the lateral frontal carina absent. In contrast, most Synergus species have a closed radial cell, the female antenna with 12 flagellomeres and complete or partially complete lateral frontal carina always present (Pujade-Villar \& Nieves-Aldrey 1990, Melika et al. 2006). Morphological peculiarities and host galls of Saphonecrus are depicted in Appendix 9.1. (Figs A71-A100). However, in Synergus there are exceptions from these character states, Synergus castaneus, S. plagiotrochi, and the recently described Synergus kawakamii Tang \& Melika from Taiwan (Schwéger et al. 2015b), are species with open or partially open radial cell of the fore wing. In these species, the female antenna with 12 flagellomeres, the notaulus is complete, reaching the anterior margin of the mesocutum, complete or incomplete lateral frontal carina present. Only one consistent morphological character is found to distinguish Saphonecrus from Synergus: the presence (Synergus) or absence (Saphonecrus) of the lateral frontal carina. The separation of this genus from Synergus has subsequently been widely questioned (Eady \& Quinlan 1963, Ritchie 1984, Pujade-Villar \& Nieves-Aldrey 1990), and the two genera have never been formally synonymised. Ritchie (1984) regarded the characters distinguishing Saphonecrus from Synergus as apomorphic, and saw Saphonecrus as a specialised monophyletic lineage within Synergus. Pujade-Villar \& Nieves-Aldrey (1990) revised the European species and maintained the genus, but also questioned its validity. We consider Saphonecrus not to be monophyletic and closely allied to Synergus (Pénzes et al. 2012, Bozsó et al. 2014, 2015, Schwéger et al. 2015a, b).

To this point 24 species of Saphonecrus were known worldwide (Pénzes et al. 2012, Bozsó et al. 2014). The WP species are associated mainly with galls on section Cerris of genus Quercus oaks, while some are associated with galls that develop on white oaks (section Quercus). The species generally have a single generation per year and emerge after overwintering in the gall, but those on evergreen oaks have at least the potential for two generations per year (Pujade-Villar \& Nieves-Aldrey 1990). The European species can be divided into three groups on the basis of their biology: (i) species with one annual generation, and associated with galls on section Quercus oaks (S. connatus (Hartig)); (ii) also univoltine species, associated with galls on section Cerris oaks (S. undulatus (Mayr), S. haimi (Mayr), and S. irani Melika \& Pujade-Villar); (iii) two Mediterranean species, with bivoltine life cycles, associated with galls on evergreen oaks of section Cerris (S. barbotini Pujade-Villar \& Nieves- 
Aldrey and S. gallaepomiformis (Boyer de Fonscolombe)) (Pujade-Villar \& Nieves-Aldrey 1990).

Four Saphonecrus species were listed for the Nearctic (Burks 1979) and some of them possess some non-typical character states for Saphonecrus, and their assignment to Saphonecrus must be examined in detail. In 2007, seven Saphonecrus species were listed for the Eastern Palearctic (Abe et al. 2007) and two species, S. serratus Weld and S. areolatus Weld, were known from the Oriental Region (Weld 1926). Also new species were described from Japan and China (Liu et al. 2012, Wang et al. 2010, Wachi et al. 2011b, Pujade-Villar et al. 2014) and recently we described 15 new species from Japan, Russia, China and Taiwan: Saphonecrus chinensis Tang \& Schwéger, S. gilvus Melika \& Schwéger, S. globosus Schwéger \& Tang, S. leleyi Melika \& Schwéger, S. lithocarpii Schwéger \& Melika, S. longinuxi Schwéger \& Melika, S. morii Schwéger \& Tang, S. nantoui Tang, Schwéger \& Melika, S. nichollsi Schwéger \& Melika, S. pachylomai Schwéger, Tang \& Melika, S. robustus Schwéger \& Melika, S. saliciniai Melika, Tang \& Schwéger, S. shanzhukui Melika \& Tang, S. symbioticus Melika \& Schwéger, and S. taitungi Schwéger, Tang \& Melika. The taxonomic assignment, data on biology and distribution for all known Saphonecrus species are given below; morphological descriptions and diagnoses are given in Schwéger et al. (2015a). Thus, currently 36 valid species of Saphonecrus are known worldwide (Table 6).

Preliminary molecular phylogenetic analysis suggests many clades within Saphonecrus, three of them include EP and WP Paleartic Saphonecrus species, too (Pénzes et al. 2012, Bozsó et al. 2014). All of the newly described EP Saphonecrus species (Schwéger et al. 2015b) are characterized distinct coxI haplotypes ("barcodes"), which support the delimitation of novel species established by the morphology. However, molecular phylogeny does not support some of morphological groups of species and Saphonecrus does not appear to be monophyletic (Bozsó et al. 2014, see also section Results 3.4.). 
Table 6. Saphonecrus species: world distribution and host associations (after Schwéger et al. 2015a)

\begin{tabular}{|c|c|c|}
\hline Species & Distribution* & Host plants \\
\hline S. areolatus Weld, 1926 & O: Philippines, Luzon & Unknown \\
\hline S. barbotini Pujade-Villar \& Nieves-Aldrey, 1985 & WP: Iberia & Quercus sect. Cerris \\
\hline S. brevicornis (Ashmead, 1896) & NA: California & Unknown \\
\hline S. chaodongzhui Melika, Ács \& Bechtold, 2004 & EP: China, Yunnan & Unknown \\
\hline S. chinensis Tang \& Schwéger, 2015 & EP: China & Lithocarpus \\
\hline S. connatus (Hartig, 1840) & WP: Europe & Quercus sect. Quercus \\
\hline S. diversus Belizin, 1968 & EP: Far East Russia & Unknown \\
\hline S. excisus (Kieffer, 1904) & EP: Bengal, Kurseong & Lithocarpus \\
\hline S. favanus Weld, 1944 & NA: DC and Missouri & Quercus sect. Lobatae \\
\hline S. flavitibilis Wang \& Chen, 2010 & EP: China, Zhejiang & Unknown \\
\hline S. gallaepomiformis (Boyer de Fonsc., 1832) & WP: Iberia & Quercus sect. Cerris \\
\hline S. gemmariae Ashmead, 1885 & NA: USA, Florida & Quercus sect. Lobatae \\
\hline S. gilvus Melika \& Schwéger, 2015 & EP: Taiwan & $\begin{array}{l}\text { Quercus subgenus } \\
\text { Cyclobalanopsis }\end{array}$ \\
\hline S. globosus Schwéger \& Tang, 2015 & EP: Taiwan & $\begin{array}{l}\text { Quercus subgenus } \\
\text { Cyclobalanopsis }\end{array}$ \\
\hline S. haimi (Mayr, 1872) & WP: Europe, N.Africa & Quercus sect. Cerris \\
\hline S. irani Melika \& Pujade-Villar, 2006 & WP: Iran & Quercus sect. Cerris \\
\hline S. leleyi Melika \& Schwéger, 2015 & EP: Russia, Far East & Quercus sect. Quercus \\
\hline S. lithocarpii Schwéger \& Melika, 2015 & EP: Taiwan & Lithocarpus \\
\hline S. longinuxi Schwéger \& Melika, 2015 & EP: Taiwan & $\begin{array}{l}\text { Quercus subgenus } \\
\text { Cyclobalanopsis }\end{array}$ \\
\hline S. morii Schwéger \& Tang, 2015 & EP: Taiwan & $\begin{array}{l}\text { Quercus subgenus } \\
\text { Cyclobalanopsis }\end{array}$ \\
\hline S. naiquanlini Melika, Ács \& Bechtold, 2004 & EP: China, Zhejiang & Unknown \\
\hline S. nantoui Tang, Schwéger \& Melika, 2015 & EP: Taiwan & $\begin{array}{l}\text { Quercus subgenus } \\
\text { Cyclobalanopsis }\end{array}$ \\
\hline S. nichollsi Schwéger \& Melika, 2015 & EP: Taiwan & Lithocarpus \\
\hline S. pachylomai Schwéger, Tang \& Melika, 2015 & EP: Taiwan & $\begin{array}{l}\text { Quercus subgenus } \\
\text { Cyclobalanopsis }\end{array}$ \\
\hline S. reticulatus Pujade-Villar, Wang \& Guo, 2014 & EP: China & Quercus sect. Quercus \\
\hline S. robustus Schwéger \& Melika, 2015 & EP: Taiwan & $\begin{array}{l}\text { Quercus subgenus } \\
\text { Cyclobalanopsis }\end{array}$ \\
\hline S. serratus Weld, 1926 & O: Philippines, Luzon & Unknown \\
\hline S. saliciniai Melika, Tang \& Schwéger, 2015 & EP: Taiwan & $\begin{array}{l}\text { Quercus subgenus } \\
\text { Cyclobalanopsis }\end{array}$ \\
\hline S. shanzhukui Melika \& Tang, 2015 & EP: Taiwan & $\begin{array}{l}\text { Quercus subgenus } \\
\text { Cyclobalanopsis }\end{array}$ \\
\hline S. shirakashii (Shinji, 1940) & EP: Japan, Taiwan & $\begin{array}{l}\text { Quercus subgenus } \\
\text { Cyclobalanopsis }\end{array}$ \\
\hline S. shirokashicola (Shinji, 1941) & EP: Japan, Taiwan & $\begin{array}{l}\text { Quercus subgenus } \\
\text { Cyclobalanopsis }\end{array}$ \\
\hline S. sinicus Belizin, 1968 & EP: China, Sichuan & Unknown \\
\hline S. symbioticus Melika \& Schwéger, 2015 & EP: Russia, Japan & Quercus sect. Quercus \\
\hline S. taitungi Schwéger, Tang \& Melika, 2015 & EP: Taiwan & $\begin{array}{l}\text { Quercus subgenus } \\
\text { Cyclobalanopsis }\end{array}$ \\
\hline S. tianmushanus Wang \& Chen, 2010 & EP: China, Zhejiang & Unknown \\
\hline S. undulatus (Mayr, 1872) & WP: Europe & Quercus sect. Cerris \\
\hline Total species number: 36 species & & \\
\hline
\end{tabular}

* O - Oriental region, WP, EP, Western and Eastern Palearctic, NA - Nearctic 


\subsubsection{Key to the Palearctic Saphonecrus species}

For the identification of all Palearctic Saphonecrus species for the first time a speciescomplete key is proposed (Schwéger et al. 2015b).

\section{Key to Palearctic Saphonecrus Dalla Torre \& Kieffer species}

1. Base of mesoscutellum nearly as broad as mesoscutum posteriorly; dorsoaxillar area narrow, inconspicuous; syntergite without row of setae anterolaterally

S. sinicus

- Base of mesoscutellum narrower than posterior width of mesoscutum, dorsoaxillar area distinct and broad; syntergite with row of short white setae anterolaterally
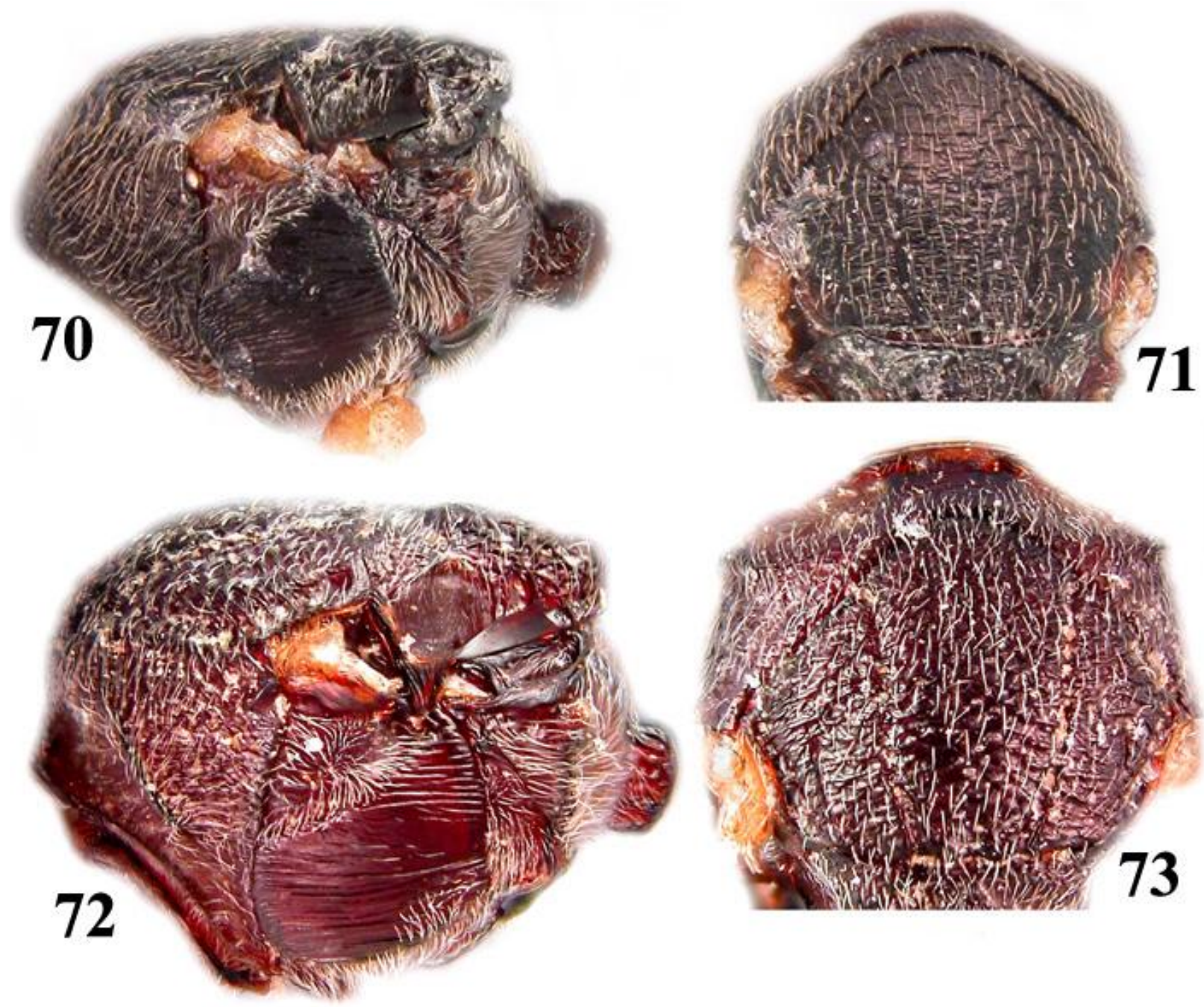

FIGURES 70-73. 70-71, Saphonecrus chaodongzhui, mesosoma, female: 70, lateral view, 71, dorsal view. 72-73, S. naiquanlini, mesosoma, female: 72, lateral view, 73, dorsal view. 
2. Lateral pronotal carina absent, anterolateral sides of pronotum rounded in dorsal view (Figs $70-71)$ 3

- Lateral pronotal carina present, complete or partially complete, anterolateral sides of pronotum usually sharply angled in dorsal view (Figs 72-73) ...

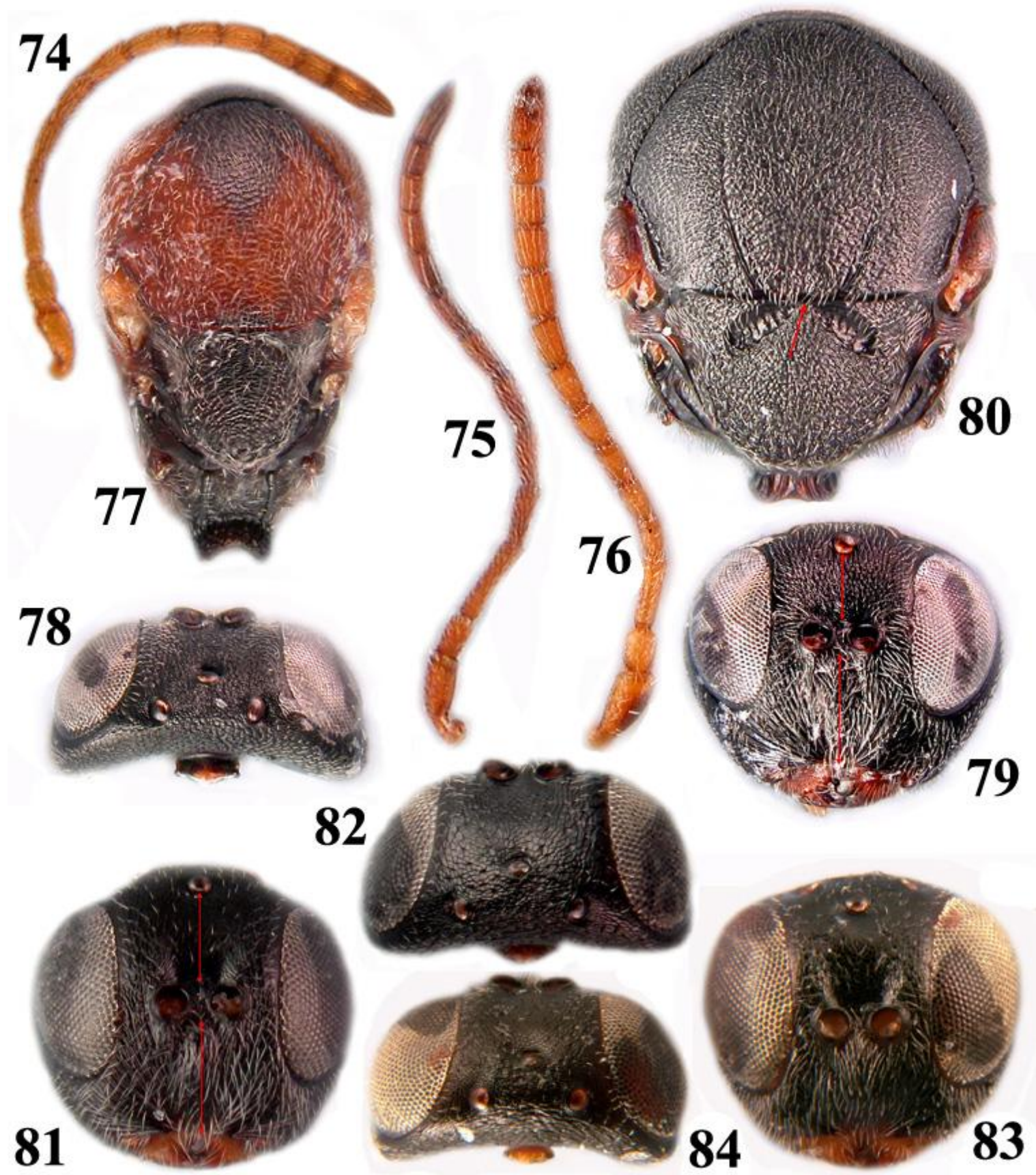

FIGURES 74-84. 74-76, antenna, female: 74, S. barbotini, 75, S. gallaepomiformis, 76, S. chaodongzhui. 77, S. gallaepomiformis, female, mesosoma, dorsal view. 78-79, S. symbioticus, female: 78, head, frontal view, 79, head, dorsal view, 80, mesosoma, dorsal view. 81-82, S. leleyi, female, head: 81, frontal view, 82, dorsal view. 83-84, S. chaodongzhui, female, head: 83 , frontal view, 84, dorsal view. 
3. Radial cell of fore wing less than $2.0 \mathrm{x}$ as long as broad S. irani* $^{*}$

- Radial cell more than $2.5 \mathrm{x}$ as long as broad ....................................... 4

4. Head, mesosoma and metasoma brown, if black than only partially ....................... 5

- Head and mesosoma black, metasoma black or dark brown ................................. 7

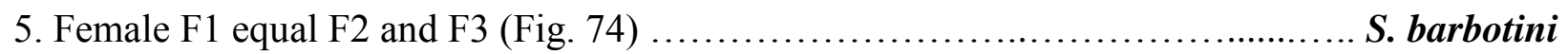

- Female F1 about 2.0x as long as F2 (Figs 75-76) ................................ 6

6. Mesoscutum uniformly dark brown to black, with distinct transverse interrupted rugae, with smooth interspaces between rugae; notaulus extending at least to half length of mesoscutum (Fig. 71)

S. chaodongzhui

- Mesoscutum reddish brown with black area only between anterior parallel lines, delicately coriaceous, without transverse interrupted rugae; notaulus present in posterior $1 / 4$ of mesoscutum length (in some specimens absent) (Fig. 77.).....

S. gallaepomiformis

7. Female antenna with 12 flagellomeres, female F1 slightly broadened apically; notaulus complete, extending to anterior margin of mesoscutum, anterior parallel line extending to $1 / 5$ of mesoscutum length

S. tianmushanus

- Female antenna with 11 flagellomeres, female F1 not broadened apically; notaulus incomplete, extending to $1 / 3-2 / 3$ of mesoscutum length, impressed only in posterior half, gradually narrowing till anterior end of mesoscutum; anterior parallel line absent ............. 8

8. Female F1 1.6-2.0x as long as F2; antenna, tibiae, tarsi pale yellow S. flavitibilis - Female F1 1.1-1.3x as long as F2; antenna, tibiae, tarsi brown 9 9. Head in dorsal view 2.1x as broad as long (Fig. 78); torulus slightly above mid height of eye, lower face 1.6x as high as height of frons (Fig. 79); notaulus complete (Fig. 80) S. symbioticus

- Head in dorsal view 1.6-1.8x as broad as long (Figs 82, 84); torulus in lower half of eye height, lower face 1.2x as high as height of frons (Figs 81, 83); notaulus incomplete (Fig. 85) 10

10. Frons with deep punctures (Figs 81-82); notaulus extending to 2/3 of mesoscutum length, gradually narrowing till anterior margin of mesoscutum (Fig. 85); scutellar foveae kidneyshaped, with smooth bottom, posteriorly well-delimited from disk of mesoscutellum; median 
area between foveae broad, fovea 2.0x as long as width of median area (Fig. 85); males unknown S. leleyi
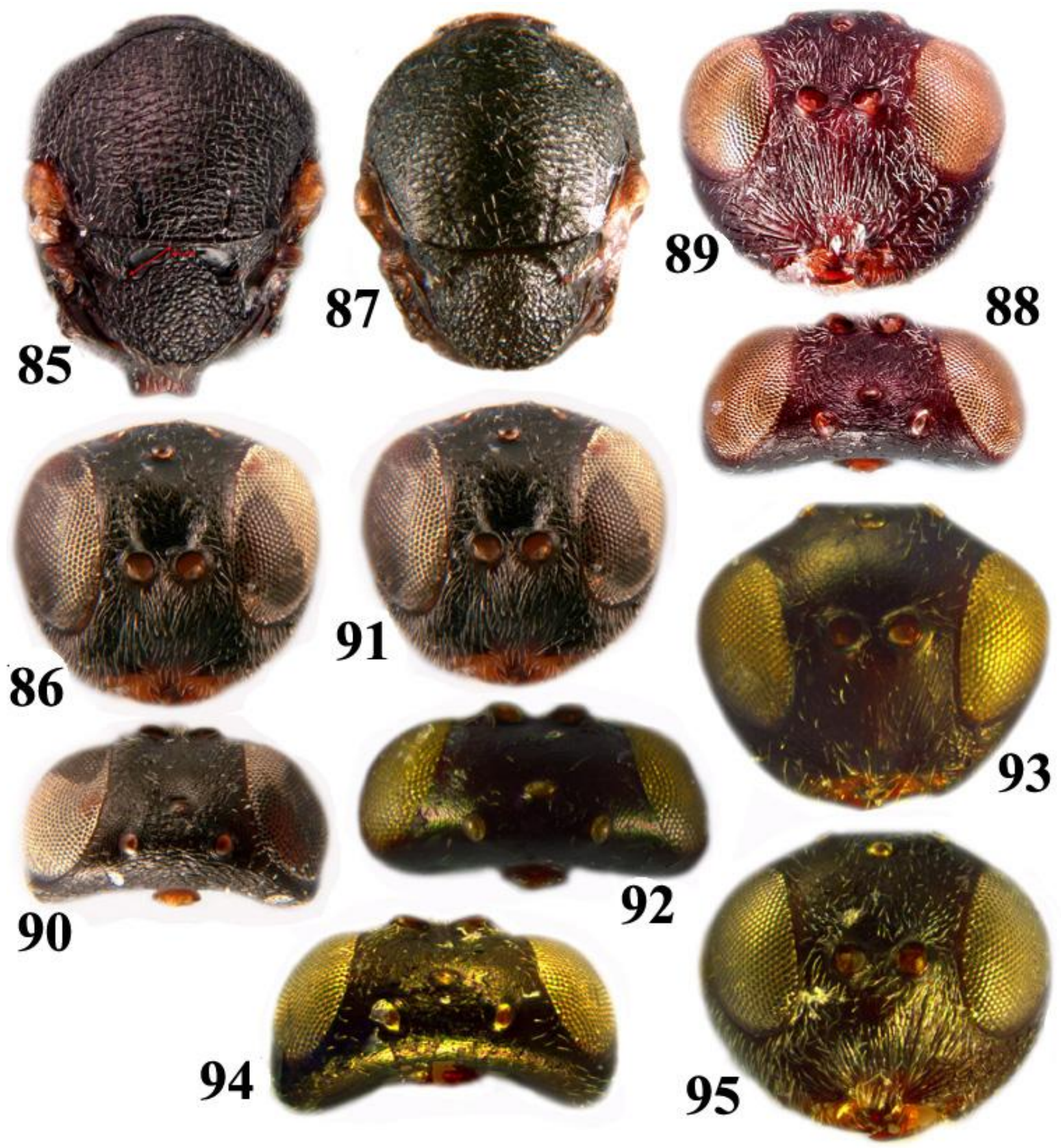

FIGURES 85-95. 85, S. leleyi, female, mesosoma, dorsal view. 86-87, S. connatus, female: 86, head, frontal view, 87, mesosoma, dorsal view. 88-89, S. naiquanlini, female, head: 88, dorsal view, 89, frontal view. 90-91, S. chinensis, female, head: 90, dorsal view, 91, frontal view. 9293, S. globosus, female, head: 92, dorsal view, 93, frontal view. 94-95, S. taitungi, female, head: 94, dorsal view, 95, frontal view. 
- Frons without punctures (Fig. 86); notaulus extending to 1/3-1/4 of mesoscutum length (Fig. 87); scutellar foveae ovate, with coriaceous bottom, posteriorly indistinctly delimited from disk; median area between foveae narrow, triangulate (Fig. 87); males known S. connatus 11. Radial cell of fore wing less than $2.0 \mathrm{x}$ as long as broad S. irani

- Radial cell more than $2.5 \mathrm{x}$ as long as broad 12

12. Notaulus uniformly impressed, complete, reaching anterior margin of mesoscutum 13 - Notaulus anteriorly less impressed than posteriorly, incomplete or absent, if incomplete than extending to half of mesoscutum length

13. Mesopectus with longitudinal striae not extending to anterior margin of mesopectus, reticulate in anterior part and between striae S. reticulatus - Mesopectus with longitudinal striae extending to anterior margin of mesopectus, area between striae smooth or delicately coriaceous 14 14. Mesoscutum with strong and long transverse rugae, especially between notauli; body length more than $2.5 \mathrm{~mm}$ 15

- Mesoscutum coriaceous, without or with delicate and short rugae; body length 1.3-2.0 mm

15. Head reddish brown, mesoscutum black or reddish brown; female F1 longer than F2; male known 16

- Head and mesoscutum black; female F1=F2; male unknown S. areolatus 16. POL 2.3x as long as OOL (Fig. 88); male known S. naiquanlini - POL 1.25x as long as OOL; male unknown S. hupingshanensis

17. Gena smooth, glabrous; scutellar foveae narrow, transversely ovate; lateral propodeal carina curved outwards anteriorly. S. diversus

- Gena alutaceous, matt; scutellar foveae as broad as high or higher than broad; lateral propodeal carinae parallel 18

18. Frons and interocellar area with delicate transverse striae (Figs 88-89) S. naiquanlini - Frons and interocellar area smooth, alutaceous or coriaceous, without transverse striae (Figs 90-91, 92-93) . 19

19. Gena broadened behind eye, visible in frontal and dorsal views (Figs 90-91, 94-95) ........ 20

- Gena not broadened behind eye, invisible in frontal and dorsal views (Figs 92-93) 22 


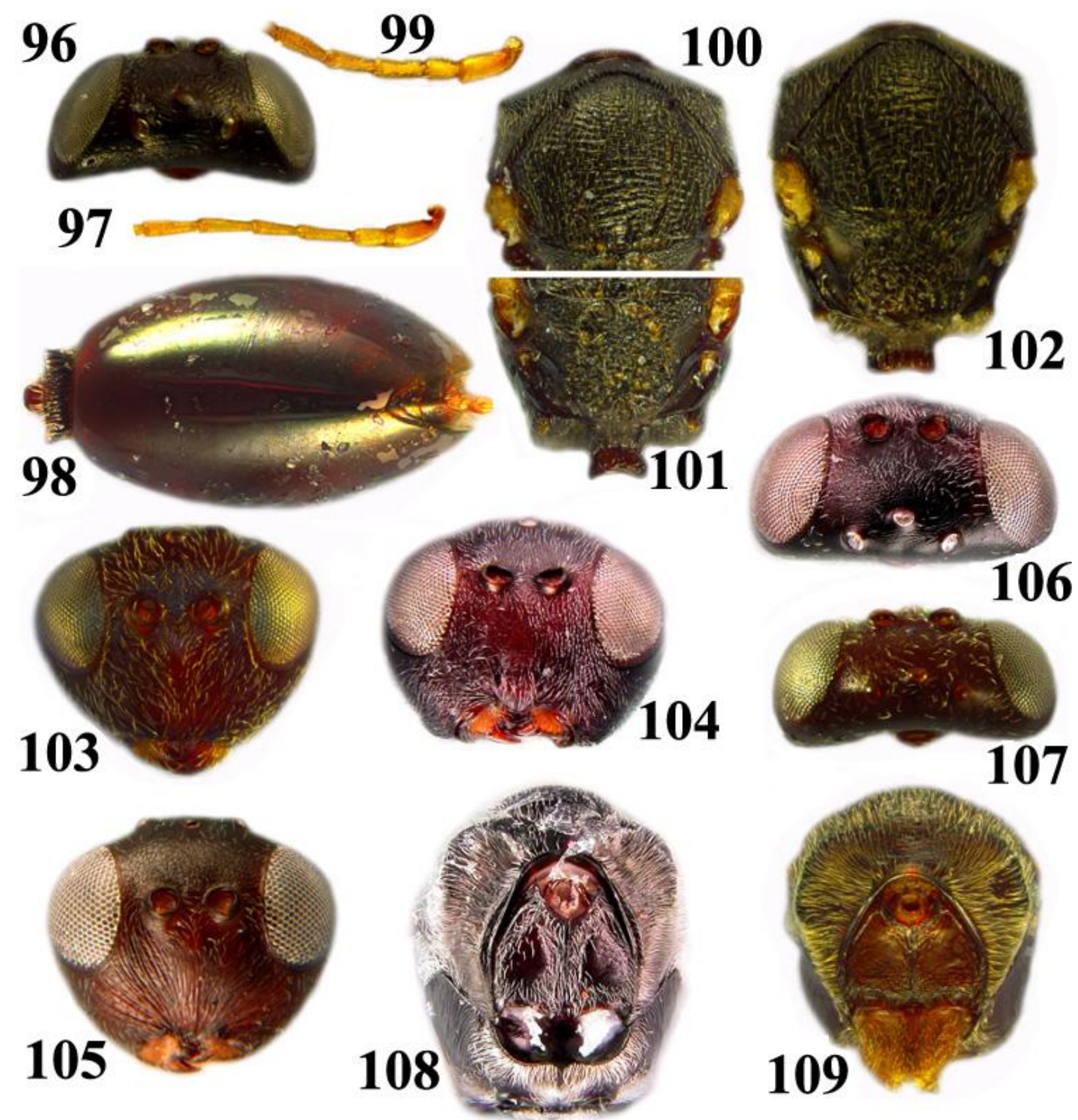

FIGURES 96-109. 96-98, S. robustus, female: 96, head dorsal view, 97, antenna, part, 98, metasoma, dorsal view. 99-101, S. chinensis, female: 99, antenna, part, 100, mesoscutum, dorsal view, 101, mesoscutellum, dorsal view. 102, S. taitungi, female, mesosoma, dorsal view. 103-105, head, frontal view: 103, S. morii, 104, S. nantoui, 105, S. gilvus. 106-107, head, dorsal view, female: 106, S. nantoui, 107, S. morii. 108-109, pronotum and propleuron, frontal view, female: 108, S. nantoui, 109, S. morii. 
20. Scutellar foveae rounded; POL 2.7x as long as OOL (Fig. 96); female F1 1.2x as long as F2 (Fig. 97); female syntergite dorsoposteriorly incised; prominent part of ventral spine of hypopygium $2.5 \mathrm{x}$ as long as broad in ventral view (Fig. 98); male unknown

S. robustus

- Scutellar foveae transverse or kidney-shaped, obliquely orientated backwards; POL 1.9-2.2x as long as OOL (Fig. 90); female F1 equal or only slightly longer than F2 (Fig. 99); female syntergite dorsoposteriorly not or very slightly incised; prominent part of ventral spine of hypopygium as long as broad in ventral view; male known ..................................... 21 21. Anterior notaular pit deep, bottom of notaulus with rugae (Fig. 100); scutellar foveae transverse (Fig. 101) S. chinensis

- Anterior notaular pit absent, bottom of notaulus smooth, without rugae (Fig. 102); scutellar foveae kidney-shaped, orientated obliquely backwards (Fig. 102) S. taitungi 22. Malar space and lower face without or with indistinct delicate striae laterally, mid part of lower face without striae (Figs 90, 103) 23

- Malar space and lower face with striae reaching torulus and extending into space between eye and torulus (Fig. 105) 24 23. Head quadrangular in frontal view, eye 1.6x as high as length of malar space (Fig. 104); POL 2.0x as long as OOL (Fig. 106); propleuron with dense setae laterally (Fig. 108); scutellar foveae rounded, nearly as high as broad; male F1 1.2x as long as F2, slightly broadened apically and basally S. nantoui - Head trapezoid in frontal view, eye $0.7 \mathrm{x}$ as high as length of malar space (Fig. 103); POL 2.5x as long as OOL (Fig. 107); propleuron without dense setae laterally (Fig. 109); scutellar foveae transverse, broader than high; male F1 $1.2 \mathrm{x}$ as long as F2, slightly broadened apically, not broadened basally S. morii 24. Pronotum rounded dorsolaterally, not sharply angled, pronotal carina short (Figs 110-111); transfacial distance 1.2x as long as height of eye (Fig. 105); female F1 1.7x as long as F2, F11 2.5x as long as F10; male F1 2.3 $\mathrm{x}$ as long as F2 S. gilvus - Pronotum sharply angled dorsolaterally, pronotal carina complete (Figs 112-113); transfacial distance equal or slightly longer than height of eye (Figs 114-115); female F1 1.2-1.4x as long as F2, F11 2.0x as long as F10; male F1 1.2-1.7x as long as F2 .................................. 25 25. Eye 1.2-1.3x as high as length of malar space (Figs 114-115); female F1 about 1.5x as long as F2 26 


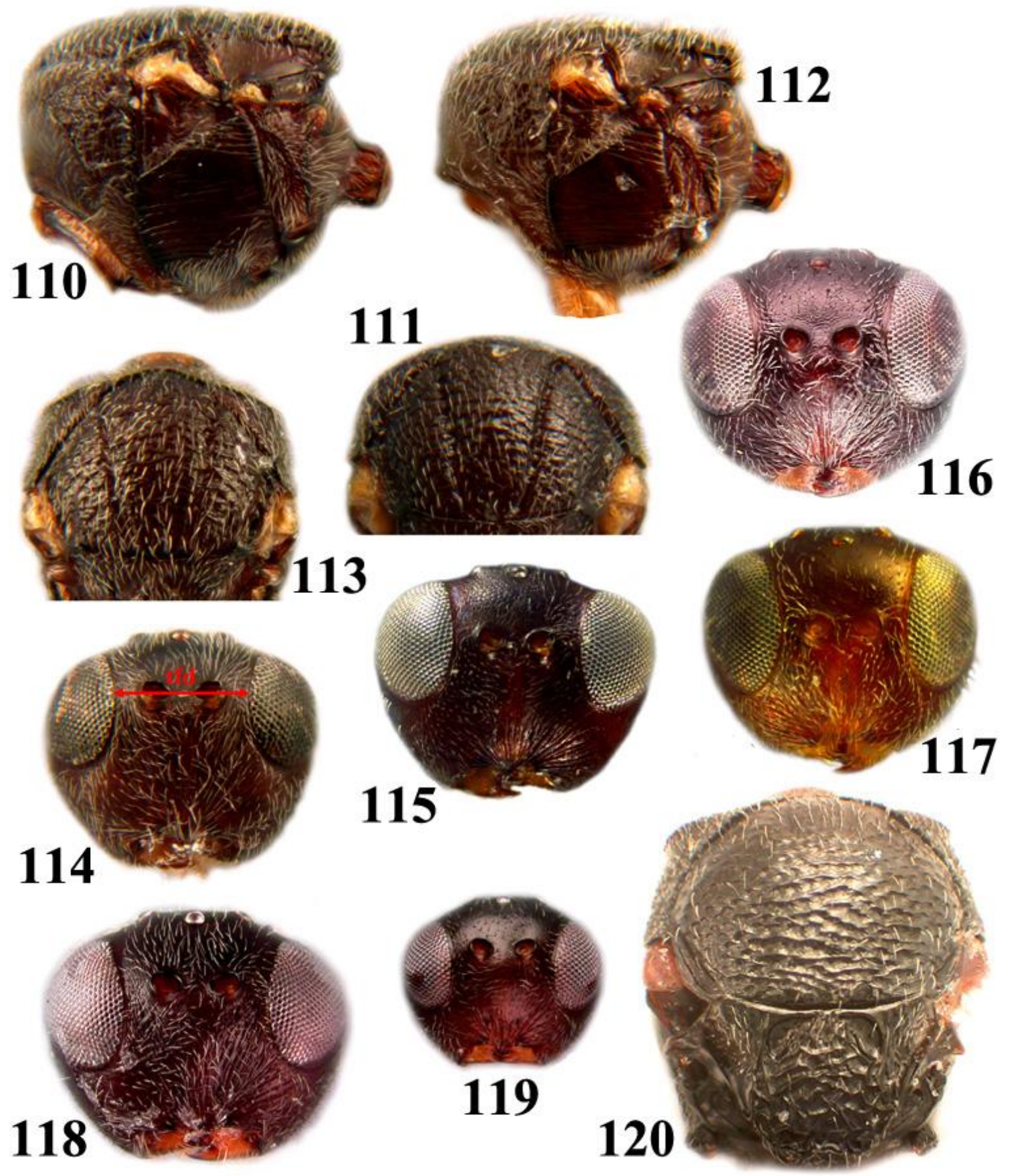

FIGURES 110-120. 110-111，S. gilvus, female: 110, mesosoma, lateral view, 111, mesoscutum, dorsal view. 112-113, S. pachylomai, female: 112, mesosoma, lateral view, 113, mesoscutum, dorsal view. 114-117, female, head, frontal view: 114, S. pachylomai, 115, S. shanzhukui. 116, S. lithocarpii, 117, S. nichollsi. 118-119, S. shirakashii, head, frontal view: 118, female, 119, male. 120, S. undulatus, female, mesosoma, dorsal view.

- Eye 1.6-2.0x as high as length of malar space (Fig. 117); female F1 less than 1.5x as long as F2 
26. Transfacial distance equal to height of eye (Fig. 115); radial cell 3.0x as long as broad; metasoma in female as high as long in lateral view, syntergite dorsoposteriorly slightly incised S. shanzhukui

- Transfacial distance longer than height of eye (Fig. 114); radial cell 3.9x as long as broad; metasoma in female $1.2 \mathrm{x}$ as long as high in lateral view, syntergite dorso-posteriorly not incised; male $\mathrm{F} 1$ curved in mid height, not broadened basally

S. pachylomai 27. Transfacial distance shorter than height of eye; lower face uniformly setose, frons with setae only laterally, along eye, central part of frons with sparse scattered setae (Figs 116-117); male head with denser setae than in female; male F1 1.2x as long as F2, broadened only apically; female metasoma not incised dorsoposteriorly 28

- Transfacial distance longer than height of eye; lower face and frons with uniform dense setae; male head with sparse whitish setae like in female (Figs 118-119); male F1 1.6-1.8x as long as F2, slightly broadened apically and basally; female metasoma slightly incised dorsoposteriorly

S. shirakashii

28. Head trapezoid, $1.3 \times$ as broad as high in frontal view (Fig. 116); scutellar foveae ovate, as broad as long; radial cell $2.7 \mathrm{x}$ as long as broad; female pedicel $1.7 \mathrm{x}$ as long as broad; male $\mathrm{F} 1$ slightly longer than $\mathrm{F} 2, \mathrm{~F} 3=\mathrm{F} 4$

S. lithocarpii

- Head rounded, as broad as high in frontal view (Fig. 117); scutellar foveae transverse, longer than broad, obliquelly orientated backwards to disk of mesoscutellum; radial cell $3.4 \mathrm{x}$ as long as broad; female pedicel $3.0 \mathrm{x}$ as long as broad; male $\mathrm{F} 1=\mathrm{F} 2$, F4 shorter than $\mathrm{F} 3$ S. nichollsi

29. Notauli absent (Fig. 120) 30

- Notauli incomplete, extending to half of mesoscutum length, anteriorly less impressed than posteriorly (Figs 121-122, 123-124)

30. Mesoscutum with strong elevated interrupted transverse rugae, area between rugae smooth, broader than width of ruga (Fig. 120); mesoscutellum with strong irregular rugae (Fig. 120) S. undulatus

- Mesoscutum without transverse rugae, coriaceous; mesoscutellum delicately coriaceous, without rugae S. haimi 

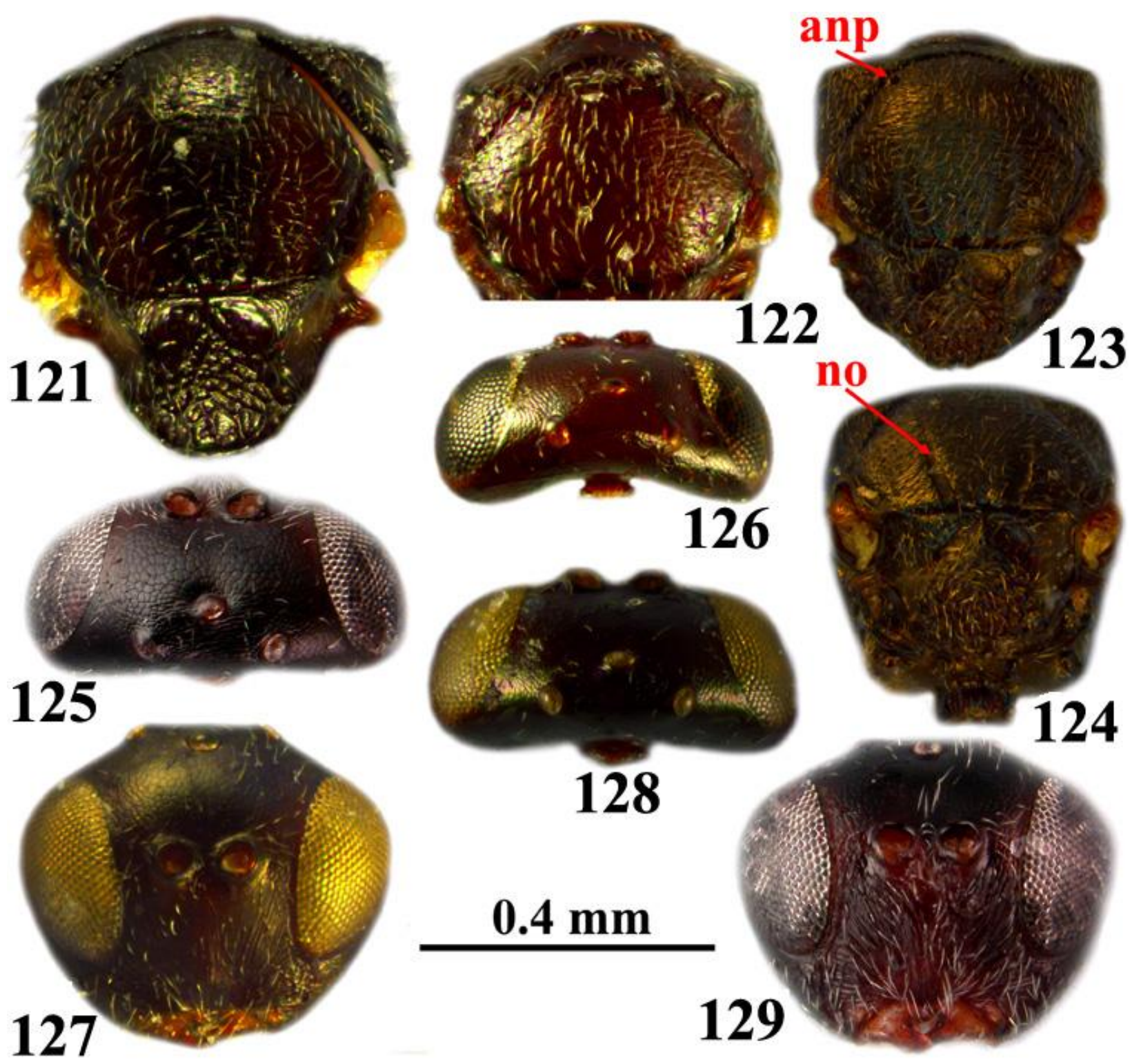

FIGURES 121-129. 121, S. globosus, female, mesosoma, dorsal view, 122, S. longinuxi, female, mesoscutum, dorsal view. 123-124, S. saliciniai, female, mesosoma: 123, dorsal view (anp, frontal notaulur pit), 124, posterodorsal view (no, notaulus). 125-126, head, dorsal view, female: 125, S. shirokashicola, 126, S. longinuxi. 127-128, S. globosus, head, female: 127, frontal view, 128, dorsal view. 129, S. shirokashicola, female, head, frontal view.

31. Anterior notaular pit absent; antenna and legs whitish; western palearctic

S. haimi

- Anterior notaular pit present (Figs 121-122), antenna and legs brown; eastern palearctic 
- POL 2.9x as long as OOL (Fig. 126); female F1 1.3x as long or equal to F2

33. Head and mesosoma black to dark brown, metasoma dark brown, dorsally darker; POL $2.3 \times$ as long as OOL (Fig. 128); transfacial distance as long as height of eye (Fig. 127)

S. globosus

- Head and mesosoma reddish brown, metasoma lighter; POL 1.9x as long as OOL (Fig. 125), transfacial distance longer than height of eye (Fig. 129)

S. shirokashicola

34. Head and mesosoma black to dark brown; female pedicel $2.1 \mathrm{x}$ as long as broad, F1 1.3x as long as F2, F11 2.1× as long as F10; male F1 1.7x as long as F2

S. saliciniai

- Head and mesosoma reddish brown; female pedicel subglobose, F1 nearly equal to F2, F11 $1.7 \times$ as long as F10; male F1 slightly longer than F2

S. longinuxi

(*S. irani is keyed out twice, with and without lateral propodeal carina; in some specimens lateral pronotal carina distinct, while in others absent or indistinct).

\subsubsection{Annotated list of worldwide Saphonecrus species}

Below we gave a worldwide Saphonecrus species list with some data on their biology, distribution, morphological peculiarities (Schwéger et al. 2015b). Species are listed in alphabetical order.

\section{Saphonecrus areolatus Weld, 1926}

A detail description of the species was given by Weld (1926). The female holotype, deposited at the United States National Museum (USNM), was examined. This species possesses the following unique morphological characters: the gena is broadened behind the eye, visible in frontal view behind the eye; the female antenna with 12 flagellomeres, F1=F2; the mesoscutum has strong and long transverse rugae, especially between the complete notauli, which reach the anterior margin of the mesoscutum; the metanotal trough has dense white setae; the lateral propodeal carinae are not parallel, slightly curved outwards in the mid height of the propodeum; tarsal claws are simple, without basal lobe; syntergite strongly incised dorsally, with a band of micropunctures extending onto lateral sides of the syntergite, and dorsally to $1 / 6$ of the syntergite length; the prominent part of the ventral spine of the hypopygium as long as broad, with a sparse long setae extending far beyond the apex of the spine. Known from Philippines (Luzon Island), host galls and host plant associations are unknown (Weld 1926). 
Saphonecrus barbotini Pujade-Villar \& Nieves-Aldrey, 1985 and Saphonecrus gallaepomiformis (Boyer de Fonscolombe, 1832)

Both species form a distinct lineage (Ács et al. 2010, see also section Results 3.4.) and associate with Mediterranean evergreen oak species ( $Q$. ilex, Q. suber, Q. coccifera), with two generations per year. They attack Plagiotrochus galls only, especially woody galls of $P$. britaniae Barbotin and P. coriaceus (Mayr) in twigs (Pujade-Villar \& Nieves-Aldrey 1990). Earlier, in all relevant literature, S. gallaepomiformis was referred to Saphonecrus lusitanicus (Tavares, 1902). After examination of types, Pujade-Villar (2004) made the adequate nomenclatorial changes and proposed the new name, Saphonecrus gallaepomiformis, which we followed (Pénzes et al. 2012).

Saphonecrus brevicornis (Ashmead, 1896)

Originally described as Synergus (Ashmead 1896). Weld (1952) transferred the species to Saphonecrus based on one character: open radial cell of the fore wing. We were unable to examine the type of S. brevicornis, thus cannot decide whether it is a Saphonecrus or a Synergus species with a partially open radial cell of the fore wing. Type must be examined to make a final decision, thus we leave it in Saphonecrus for now.

Saphonecrus chaodongzhui Melika, Ács \& Bechtold, 2004

In the original description of this species the presence of weak lateral frontal carina was erroneously indicated (Melika et al. 2004) which, in fact, is absent. Host cynipid galls and host plant associations are unknown. Known from China, (Yunnan, Diqing, Xiaozhongdian), later was found also in Zhejiang Province of China (Wang et al. 2010).

Saphonecrus chinensis Tang \& Schwéger, 2015

This species was reared from unknown round bud galls, flattened on top, up to $15 \mathrm{~mm}$ in diameter, with small depression in top surface at centre of the gall; the gall is green when fresh, turning brown when matures (Fig. A85 in Appendix 9.1.); collected from Lithocarpus fenestratus. Adults emerged under laboratory conditions during late April. Currently known only from China (Lan Cang County). Diagnosis and detail species description are given in Schwéger et al. (2015b). 
Saphonecrus connatus (Hartig, 1840)

Saphonecrus connatus is thought to be a trans-Palearctic species and was mentioned from Japan and Korea, reared from leaf galls on Q. dentata (Sakagami 1949, Abe et al. 2007). However, this record might be well S. chaodongzhui Melika, Ács \& Bechtold, known from China, which closely resembles $S$. connatus (Melika et al. 2004). The most peculiar character of $S$. connatus is the absence of notaulus. This species attacks some Andricus species and galls of Callirhytis glandium (Giraud), Cynips quercusfolii (L.), Neuroterus anthracinus (Curtis) and $N$. quercusbaccarum (L.) (Pénzes et al. 2009, Pujade-Villar \& Nieves-Aldrey 1990, Pujade-Villar et al. 2003).

\section{Saphonecrus diversus Belizin, 1968}

Based on the original description it is a Saphonecrus (Belizin 1968). The species was described on the basis of three females. According to Belizin (1968) most closely resembles S. undulatus. Known from the Far East of Russia, host galls and host plant associations are unknown (Belizin 1968). We were unable to obtain the type of this species, thus it is still need a detailed examination.

\section{Saphonecrus excisus (Kieffer, 1904)}

Based on the description (Dalla Torre \& Kieffer 1910) it is a Saphonecrus. We tried for several years to locate the type of the species unsuccessfully and the type might be well lost. The species is known from Kurseong, West Bengal, India, reared from stem swelling-like galls of Neuroterus haasi Kieffer, collected from Lithocarpus elegans. Based on the host plant association, it is possible that this species belongs to a supposed Saphonecrus clade which associates exclusively with Lithocarpus (Bozsó et al. 2014, section Results 3.4.).

\section{Saphonecrus favanus Weld, 1944}

This species is known from the U.S.A. (Washington DC and Missouri), reared from a root gall of Dryocosmus favus Beutenmüller on section Lobatae of genus Quercus (red oaks) (Weld 1944). The type female was examined by us. Morphologically it is not a typical Saphonecrus: the frons, vertex and mesoscutum have numerous deep punctures (resembling Synergus subterraneus 
Giraud); the head is quadrangular in the frontal view, the clypeus is impressed, the lower face is delicately coriaceous, without radiating striae; the female antenna has 11 flagellomeres; the lateral frontal and lateral pronotal carina are absent; the fore wing margin lacks cilia; the metapleural sulcus reaches the posterior margin of the mesopectus in the upper $1 / 3$ of its height; the first metasomal tergite has parallel sulci laterally and dorsally; the last metasomal tergite has a posterodorsal patch of micropunctures. Saphonecrus favanus might represent a distinct, undescribed genus. More specimens and further examination are in need.

Saphonecrus flavitibilis Wang \& Chen, 2010

Known from China (Zhejiang), host galls and host plant associations are unknown (Wang et al. 2010).

Saphonecrus gemmariae Ashmead, 1885

The species was described from Florida, based on one male, which emerged from Callirhytis quercusgemmariae (Ashmead) gall on red oaks (Ashmead 1885). The type supposed to be deposited at the USNM, however, was neither located in the collection by the curator, M. Buffington nor by G. Melika. The description of the male is very brief, not enough detail to make a decision whether S. gemmariae is a Saphonecrus or a Synergus.

Saphonecrus gilvus Melika \& Schwéger, 2015

This species was reared from undescribed hairy round galls on leaf midribs (Fig. A86) on $Q$. gilva. Galls were collected in November from which adults emerged under laboratory conditions during November. Currently it is known only from Taiwan (Taichung County). Diagnosis and detail species description are given in Schwéger et al. (2015b).

Saphonecrus globosus Schwéger \& Tang, 2015

This species was reared from undescribed leaf galls (Fig. A87) on Q. globosa in May. Adults emerged under laboratory conditions in May. Currently known only from Taiwan (Nantou County). Diagnosis and detail species descriptions are given in Schwéger et al. (2015b). 
Three species, Saphonecrus haimi (Mayr, 1872), Saphonecrus irani Melika \& Pujade-Villar, 2006, Saphonecrus undulatus (Mayr, 1872), which have one generation per year, attack galls of Aphelonyx cerricola (Giraud), Cerroneuroterus lanuginosus (Giraud), Chilaspis nitida (Giraud), Ch. israeli (Sternlicht), Pseudoneuroterus saliens (Kollar) and galls modified by Synophrus politus (Pujade-Villar et al. 2003, Melika 2006), all of which are associating with Quercus section Cerris only.

Saphonecrus leleyi Melika \& Schwéger, 2015

Wasps emerged from undescribed bud galls (Fig. A97) on Q. mongolica in late September and also from the asexual galls of Andricus mukaigawae (Pujade-Villar et al. 2016). Known from Far East of Russia and China (Pujade-Villar et al. 2016). Diagnosis and detailed species description are given in Schwéger et al. (2015b).

Saphonecrus lithocarpii Schwéger \& Melika, 2015

This species was reared from undescribed bud galls (Fig. A87) on Lithocarpus glabra and leaf galls (Fig. A88) on L. konishii. Galls were collected in late autumn and beginning of January from which adults emerged under laboratory conditions from January till April. Currently it is known only from Taiwan (Taichung City, Nantou County). Diagnosis and detail species description are given in Schwéger et al. (2015b).

Saphonecrus longinuxi Schwéger \& Melika, 2015

This species was reared from undescribed small round galls on the upper surface of the leaves (Fig. A87) of Q. longinux in early March, from which adults emerged by the end of April. Currently it is known only from Taiwan (Nantou County). Diagnosis and detail species description are given in Schwéger et al. (2015b).

Saphonecrus morii Schwéger \& Tang, 2015

This species was reared from leaf galls (Fig. A91) on Q. morii in February and later in May. Adults emerged immediately after they were put onto laboratory rearing. Currently known only from Taiwan (Nantou County). Diagnosis and detail species description are given in Schwéger et al. $(2015 b)$. 
Saphonecrus naiquanlini Melika, Ács \& Bechtold, 2004

Originally described from China (Zhejiang), the host cynipid gallwasp species and host plants are unknown (Melika et al. 2004). Later, it was collected in Yunnan Province of China (Wang et al. 2010).

Saphonecrus nantoui Tang, Schwéger \& Melika, 2015

This species was reared from undescribed leaf petiole thickening galls (Fig. A92) on Quercus glauca (Thunb.) Oerst. Galls were collected in late February from which adults emerged under laboratory conditions at the beginning of March. Currently known only from Taiwan (Nantou County). Diagnosis and detailed species description are given in Schwéger et al. (2015b).

Saphonecrus nichollsi Schwéger \& Melika, 2015

This species was reared from undescribed stem swelling-like galls (Fig. A93) on L. konishii in October, from which adults emerged immedeately after the galls were put onto laboratory rearing. Currently known only from Taiwan (Taichung City). Diagnosis and detailed species description are given in Schwéger et al. (2015b).

Saphonecrus pachylomai Schwéger, Tang \& Melika, 2015

This species was reared from undescribed stem swelling-like galls (Fig. A95) on Q. pachyloma in late October. Adults emerged under laboratory conditions in November. Currently known only from Taiwan (Nantou County). Diagnosis and detail species description are given in Schwéger et al. (2015b).

Saphonecrus reticulatus Pujade-Villar, Wang \& Guo, 2014

This species was reared from rounded stem swelling galls on Q. aliena var. acutiserrata (Quercus section Quercus of genus Quercus). Inquilines emerged immediately after the galls were collected in late June in China (Zhejiang Province) (Pujade-Villar et al. 2014a). One unique character differentiates this species from all other Saphonecrus species: the reticulate anterior part of the mesopectus and the reticulate surface between longitudinal striae on the mesopectus (Pujade-Villar et al. 2014a). 
Saphonecrus robustus Schwéger \& Melika, 2015

This species was reared from undescribed Dryocosmus multilocular stem swelling-like galls (Fig. A100) on Q. hypophaea in January-March. Adults emerged immedeately after they were put in laboratory rearing. Currently known only from Taiwan (Taitung County). Diagnosis and detail species description are given in Schwéger et al. (2015b).

Saphonecrus saliciniai Melika, Tang \& Schwéger, 2015

This species was reared from small egg-shaped galls on leaf midribs (Fig. A99) on Q. salicinia in mid-February. Adults emerged under laboratory conditions by the end of March. Currently known only from Taiwan (Taipei vicinity). Diagnosis and detail species description are given in Schwéger et al. (2015b).

\section{Saphonecrus serratus Weld, 1926}

The type was examined. The next characters differentiate this species from all other Saphonecrus species: the head is transverse in dorsal view, the female antenna has 12 flagellomeres, the female F1 is $1.2 \mathrm{x}$ as long as F2; the lateral pronotal carina is weak and incomplete, the sides of the pronotum are rounded in dorsal view, the mesoscutum has strong transverse rugae, the tarsal claws are simple and without basal lobe; the propodeal carinae curve outwards and are not parallel; the first metasomal tergite is not straight as in other Saphonecrus species but sinuate, the sulci are parallel, longitudinal, present dorsally and laterally; the syntergite has a posterodorsal patch of micropunctures; the prominent part of the ventral spine of the hypopygium is at least $2.0 \mathrm{x}$ as long as broad in ventral view; body length $2.7 \mathrm{~mm}$. Known from Philippines (Luzon Island), host galls and host plant associations are unknown (Weld 1926).

Saphonecrus shanzhukui Melika \& Tang, 2015

This species was reared from rounded stem swelling-like galls (Fig. A96) on Q. hypophaea. Galls were collected in January-March from which adults emerged under laboratory from January till March. Currently is known only from Taiwan (Taitung County). Diagnosis and detail species description are given in Schwéger et al. (2015b). 
Saphonecrus shirakashii (Shinji, 1940) and Saphonecrus shirokashicola (Shinji, 1941). These two species, originally described in Andricus Hartig, were erroneously placed in Ufo (Wachi et al. 2011b) but were later transferred to Saphonecrus (Melika et al. 2012). Saphonecrus shirakashii and S. shirokashicola are known from Japan and Taiwan, were reared from undescribed leaf galls on $Q$. glauca, Q. globosa and $Q$. longinux (Quercus subgenus Cyclobalanopsis) (Melika et al. 2012). Both species are characterized by having an ovate or quadrangular head in frontal view; the frons is smooth or alutaceous, without or with some delicate indistinct striae; the male F1 is $1.5 \times$ as long as F2, or only slightly longer than F2; the mesoscutum is alutaceous to delicately coriaceous, without distinct short irregular transverse striae. In S. shirakashii, the pedicel is $2.2 \times$ as long as broad; the female F1 is $1.2 \times$ as long as F2; F11 is $1.9 \times$ as long as F10; the notaulus is complete, reaching the anterior margin of the mesoscutum, while in S. shirokashicola, the pedicel is $1.6 \times$ as long as broad; the female F1 is $1.7 \times$ as long as F2; F11 is $2.3 \times$ as long as F10; the notaulus is incomplete, present only in the posterior $1 / 3-1 / 2$ of the mesoscutum. Molecular phylogeny suggests that the two species belong to different clades within Synergini (Bozsó et al. 2014, section Results 3.4.).

\section{Saphonecrus sinicus Belizin, 1968}

Based on the original description, this species is a Saphonecrus (Belizin 1968). The species was originally described on the basis of one female. According to Belizin (1968), this species differs from all other known Saphonecrus by the broad mesoscutellum, the width of which is equal to the width of the mesoscutum. The species was described from China (Sichuan); host galls and host plant associations are unknown (Belizin 1968). We were unable to locate and to obtain the type of this species, thus it is still need a detail examination.

Saphonecrus symbioticus Melika \& Schwéger, 2015

All wasps emerged exclusively from the asexual galls of Andricus hakonensis (=A.symbioticus) (Fig. A98) on Q. dentata and Q. mongolica in late September-October. Currently known only from Russia (Far East, Primorskij Kraj) and Japan (Hokkaido). Diagnosis and detailed species description are given in Schwéger et al. (2015b). 
Saphonecrus taitungi Schwéger, Tang \& Melika, 2015

This species was reared from galls, collected from L. dodoniifolius at the beginning of March, from which adults emerged under laboratory conditions by the end of March. Currently known only from Taiwan (Taitung County). Diagnosis and detail species description are given in Schwéger et al. (2015b).

Saphonecrus tianmushanus Wang \& Chen, 2010

The female of this species has an antenna with 12 flagellomeres, the suture between F12 and F11 is distinct. Described from China (Zhejiang), host galls and host plants are unknown (Wang et al. 2010).

\subsection{Synergus Hartig, 1840}

\subsubsection{General comments}

Synergus is the most species-rich inquilines genus of oak gall cynipid, with 118 known species world-wide (Table 2), with a long and complex history of taxonomic revision (see Pujade-Villar et al. 2003, Melika 2006). Currently 19 Synergus species are known from the EP including eight species recently described from Far East Russia, Japan, China and Taiwan (Sadeghi et al. 2006, Bernardo et al. 2013, Abe et al. 2011, Pujade-Villar et al. 2014, Schwéger et al. 2015a; Table 7). In the last decade, two Synergus species were described from the EP for which the host plant associations are other than Quercus subgenus Quercus. One Synergus species, S. itoensis Abe, Ide \& Wachi, appeared to induce its own gall in the seed coat of the acorn of Quercus (subgen. Cyclobalanopsis) glauca, a unique behaviour for inquilines when the species have not lost the capability to induce its own gall (Abe et al. 2011). Synergus castaneus Pujade-Villar, Bernardo \& Viggiani, described from China, is the first known cynipid inquiline (Synergini) that emerge from Castanea galls (Fagaceae) (but not from the well-known gall of Dryocosmus kuriphilus Yasumatsu) (Bernardo et al. 2013). A new species, Synergus kawakamii, is the first Synergus known to associate with galls developing on a Castanopsis species (Fagaceae) (Schwéger et al. 2015a). Recently, Synergus jezoensis Uchida \& Sakagami was revised and validated (Pujade-Villar et al. 2014); however, the status of four other EP species described in the first half of 20th century (S. atamiensis Ashmead, S. hakonensis Ashmead, S. iwatensis Shinji, and S. mizunarae Shinji) were still uncertain (Abe et al. 2007). Partially it was resolved in Schwéger et al. (2015a). 
Table 7. Eastern Palearctic Synergus species with distribution and host associations (after Schwéger et al. 2015a)

\begin{tabular}{|c|c|c|c|}
\hline Species & Distribution & Host gall & Host plant \\
\hline S. abei Melika \& Schwéger & Japan & $\begin{array}{l}\text { unknown acorn and } \\
\text { bud galls }\end{array}$ & $\begin{array}{l}\text { Quercus } \\
\text { (subgen. Quercus) }\end{array}$ \\
\hline S. belizinellus Schwéger \& Melika & Russia Japan & $\begin{array}{l}\text { Belizinella vicina } \\
\text { and unknown leaf } \\
\text { galls }\end{array}$ & $\begin{array}{l}\text { Quercus } \\
\text { (subgen. Quercus) }\end{array}$ \\
\hline $\begin{array}{l}\text { S. castaneus } \\
\text { Pujade-Villar, Bernardo \& Viggiani }\end{array}$ & China & unknown galls & Castanea \\
\hline S. changtitangi Melika \& Schwéger & Taiwan & $\begin{array}{l}\text { unknown stem } \\
\text { swellin-like galls }\end{array}$ & $\begin{array}{l}\text { Quercus } \\
\text { (subgen. Quercus) }\end{array}$ \\
\hline $\begin{array}{l}\text { S. chinensis } \\
\text { Melika, Ács \& Bechtold }\end{array}$ & $\begin{array}{l}\text { China, S.Korea } \\
\text { Russia }\end{array}$ & $\begin{array}{l}\text { unknown acorn, } \\
\text { bud and leaf galls }\end{array}$ & $\begin{array}{l}\text { Quercus } \\
\text { (subgen. Quercus) }\end{array}$ \\
\hline $\begin{array}{l}\text { S. deqingensis } \\
\text { Pujade-Villar,Wang \& Chen }\end{array}$ & China & Unknown & unknown \\
\hline S. formosanus Schwéger \& Melika & Taiwan & $\begin{array}{l}\text { Trichagalma } \\
\text { formosana }\end{array}$ & $\begin{array}{l}\text { Quercus } \\
\text { (subgen. Quercus) }\end{array}$ \\
\hline S.gifuensis Ashmead & Japan Russia & $\begin{array}{l}\text { Andricus } \\
\text { kashiwaphilus, A. } \\
\text { mukaigawae }\end{array}$ & $\begin{array}{l}\text { Quercus } \\
\text { (subgen. Quercus) }\end{array}$ \\
\hline S. hakonensis Ashmead & Japan & Unknown & unknown \\
\hline S. ishikarii Melika \& Schwéger & Japan & $\begin{array}{l}\text { Ussuraspis sp., } \\
\text { unknown leaf galls }\end{array}$ & $\begin{array}{l}\text { Quercus } \\
\text { (subgen. Quercus) }\end{array}$ \\
\hline S. itoensis Abe, Ide \& Wachi & Japan & $\begin{array}{l}\text { gallinducer } \\
\text { in acorns }\end{array}$ & $\begin{array}{l}\text { Quercus (subgen. } \\
\text { Cyclobalanopsis) }\end{array}$ \\
\hline S. iwatensis Shinji & \multicolumn{3}{|c|}{ status uncertain, nomen nudum } \\
\hline S. japonicus Walker & $\begin{array}{l}\text { Japan China } \\
\text { Russia }\end{array}$ & $\begin{array}{l}\text { Andricus } \\
\text { kashiwaphilus, A. } \\
\text { mukaigawae }\end{array}$ & $\begin{array}{l}\text { Quercus } \\
\text { (subgen. Quercus) }\end{array}$ \\
\hline S. jezoensis Uchida \& Sakagami & Japan China & Unknown & unknown \\
\hline S. kawakamii Tang \& Melika & Taiwan & $\begin{array}{l}\text { unknown detachable } \\
\text { stem galls }\end{array}$ & Castanopsis \\
\hline S. khazani Melika \& Schwéger & Russia Japan & $\begin{array}{l}\text { Andricus } \\
\text { kashiwaphilus, } A . \\
\text { mukaigawae; } \\
\text { unknown acorn and } \\
\text { bud galls }\end{array}$ & $\begin{array}{l}\text { Quercus } \\
\text { (subgen. Quercus) }\end{array}$ \\
\hline S. mizunarae Shinji & \multicolumn{3}{|c|}{ status uncertain, nomen nudum } \\
\hline S. symbioticus Schwéger \& Melika & Russia Japan & $\begin{array}{l}\text { Andricus hakonensis, } \\
\text { A. kashiwaphilus }\end{array}$ & $\begin{array}{l}\text { Quercus } \\
\text { (subgen. Quercus) }\end{array}$ \\
\hline $\begin{array}{l}\text { S. xialongmeni } \\
\text { Melika, Ács \& Bechtold }\end{array}$ & China & $\begin{array}{l}\text { unknown acorn, leaf } \\
\text { and bud galls }\end{array}$ & $\begin{array}{l}\text { Quercus } \\
\text { (subgen. Quercus) }\end{array}$ \\
\hline
\end{tabular}

The presence/absence of the lateral frontal carinae and/or lateral pronotal carina, open or closed radial cell of the fore wing, and 11 or 12 flagellomeres in female antennae in Saphonecrus 
(Synergus) are inconsistent character states, which in some cases make precise morphology based generic/specific identification uncertain and, thus, the establishment of unique set of character state for definition (at a generic level) is complicated. Morphological peculiarities and host galls of Synergus are depicted in Appendix 9.1. (Figs A101-A127). All of the newly described EP Synergus species described by us are supported by distinct coxl haplotypes supporting the morphology-based delimitation of species (Schwéger et al. 2015a).

\subsubsection{Key to Eastern Palearctic Synergus species}

For the identification of EP Synergus species the following key is proposed (Schwéger et al. 2015a).

\section{Key to Eastern Palearctic Synergus species}

1. Radial cell of fore wing entirely closed (Fig. 130) …...................................... 3

- Radial cell of fore wing partially or entirely open (Fig. 131) ................................... 2

2. Radial cell of fore wing entirely open, R1 and Rs do not reach wing margin S. castaneus

- Radial cell partially opened, R1partially running along wing margin S. kawakamii

3. Micropunctures on posterior end of syntergite extending laterally to ventral edge, forming a band around syntergite (Figs 132-133)

- Micropunctures restricted to a small posterodorsal patch, never extending laterally (Figs 134135)

4. Lateral frontal carinae very indistinct, visible only near toruli; F1 in female weakly curved medially and slightly expanded apically; F1 in male strongly expanded apically and basally, 3.0 times as long as pedicel

S. deqingensis

- Lateral frontal carinae always present, distinct (Figs 136-138); F1 in female not expanded apically, straight, cylindrical; F1 in male expanded only apically, if basally also than always less

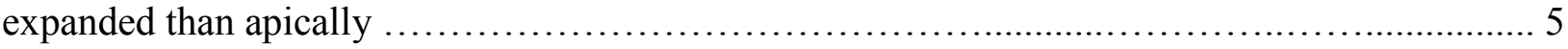

5. Syntergite without antero-lateral patch of setae; lower face in female yellow, F2 in female slightly incised medially

S. jezoensis 

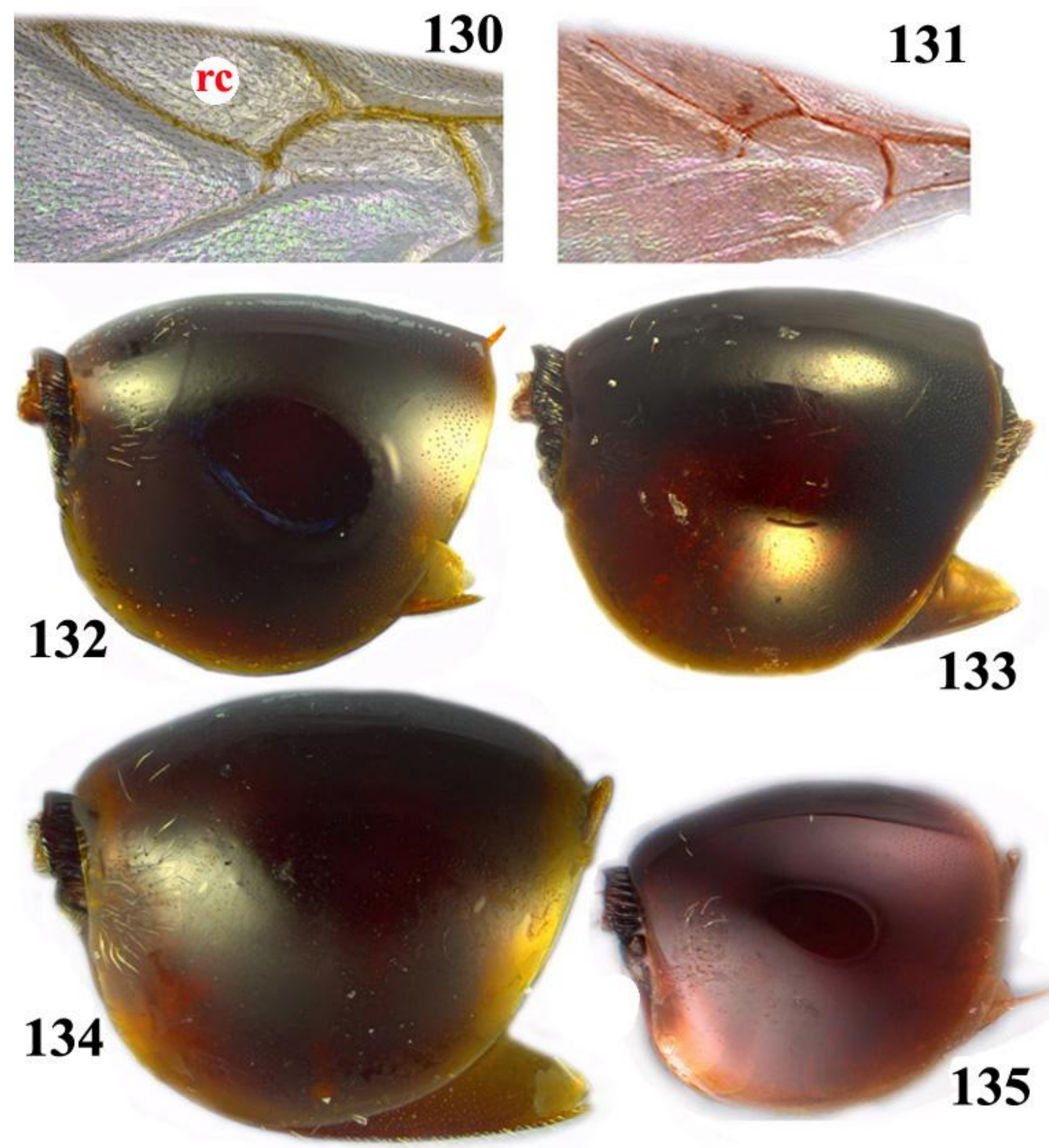

FIGURES 130-135. 130-131, female, fore wing, part (rc, radial cell): 130, Synergus abei, 131, S. kawakamii. 132-135, metasoma, female, lateral view: 132, S. abei, 133, S. changtitangi, 134, S. belizinellus, 135, S. formosanus. 
- Syntergite with antero-lateral patch of setae, lower face in female black, dark brown or reddish brown, never yellow, F2 in female not incised medially, straight (Figs 139-140, 143) .......... 6 6. F1 in female 3.0-3.3 times as long as pedicel, 1.25x longer than F2 (Figs 139, 142-143); F1 in male expanded only apically, if expanded basally than only very slightly (Figs 141, 144-145)

- F1 in female about 2.0 times as long as pedicel, subequal with F2; F1 in male expanded apically and basally .....

7. Posterior band of micropunctures on syntergite broad, dorsally extending to $1 / 3$ of its length; F1 of male expanded only apically (Figs 141, 144) 8

- Posterior band of micropunctures on syntergite narrow, dorsally extending to $1 / 5-1 / 7$ of its length; F1 of male expanded apically and very slightly basally S. abei 8. Female head transverse in dorsal view, quadrangular in frontal view (Fig. 137); frontal carina uniformly strong, always reaches lateral ocellus (Fig. 137); F1 in male strongly expanded apically (Fig. 145)

S. khazani

- Female head more robust in dorsal view (Fig. 147), rounded in frontal view (Fig. 146); frontal carina weak, sometimes hardly or not traceable at lateral ocellus or extending to $1 / 2-2 / 3$ of distance to lateral ocellus (Figs 146-147); F1 in male very weakly expanded apically (Fig. 142) S. changtitangi

9. Head ovate in frontal view; lower face with strong median carina raised above striae; pedicel in female longer than broad; median mesoscutal impression short, reaching to mid length of mesoscutum and sometimes abruptly broadened posteriorly; scutelar foveae rugose; female syntergite only slightly incised dorsoapically

S. gifuensis

- Head triangular in frontal view; lower face with weak median carina not raised above striae; female pedicel as long as broad; median mesoscutal impression almost complete; scutellar foveae alutaceous; female syntergite strongly incised dorso-apically S. japonicus 10. Head in frontal view in females and males, lower space, frons genae partially or entirely, always black or very dark brown; mesosoma black or dark brown 11 - Head in frontal view in females and males, lower space, frons and genae partially or entirely, always light brown or yellowish; mesosoma predominantly reddish brown, never black 13 


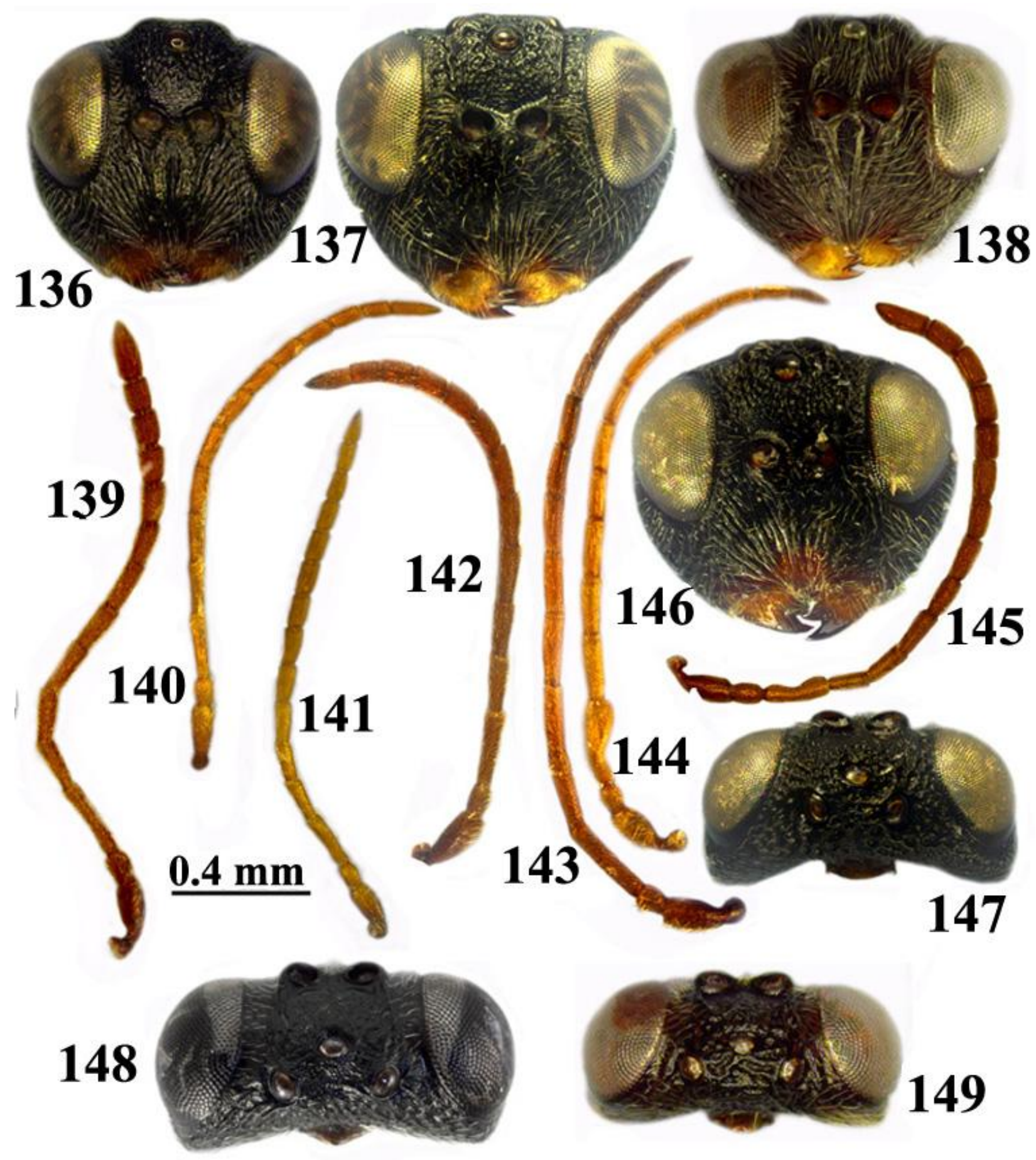

FIGURES 136-149. 136-138, head, female, frontal view: 136, S. abei, 137, S. khazani, 138, S. belizinellus. 139-145, antenna: 139, S. abei, female; 140, S. belizinellus, 141, S. changtitangi, female, 142, S. changtitangi, male, 143, S. khazani, female, 144, S. khazani, male, 145, S. abei, male. 146-147, S. changtitangi, head, female: 146, frontal view, 147, dorsal view. 148-149, head, female, dorsal view: 148, S. ishikarii, 149, S. belizinellus. 


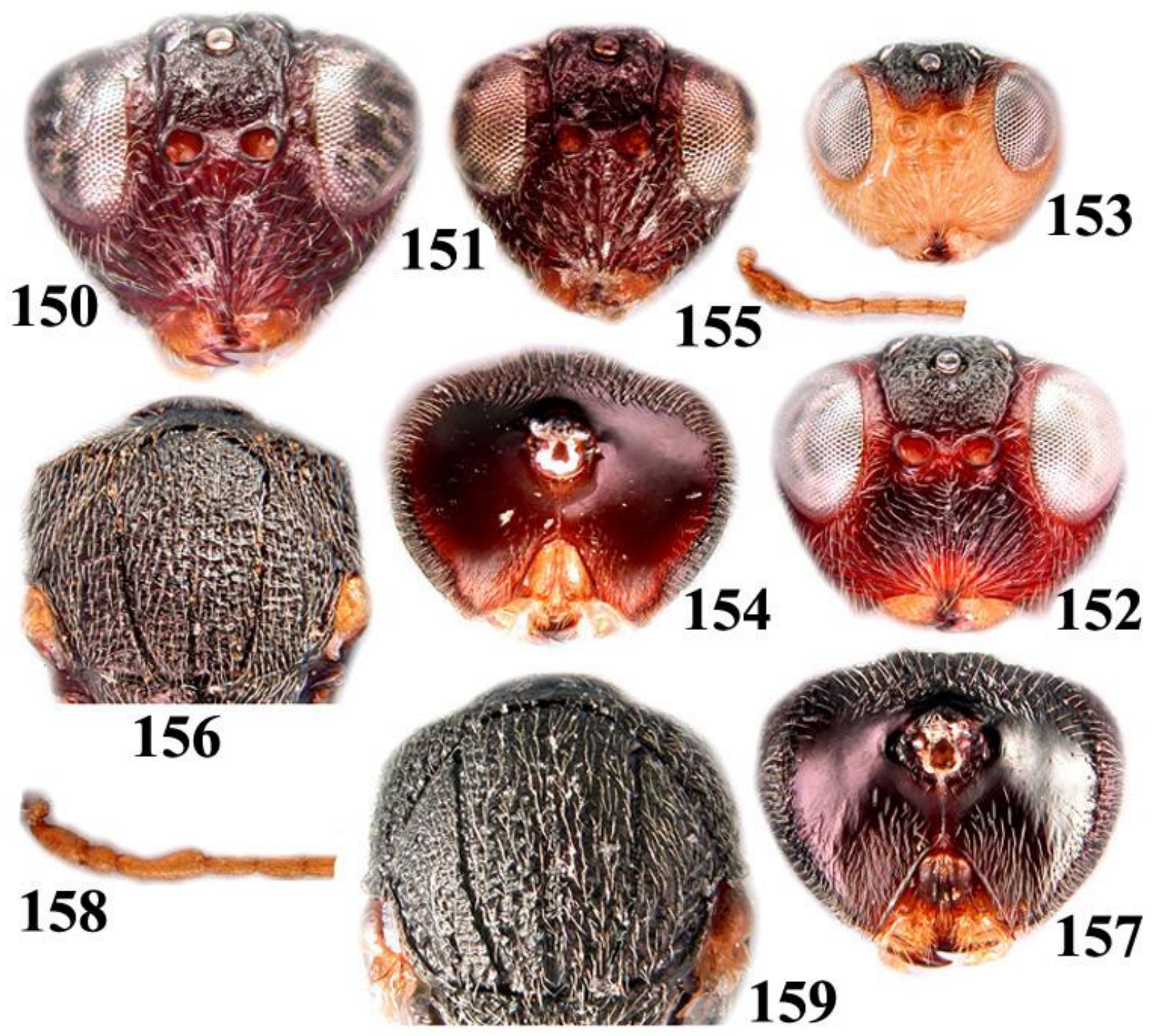

FIGURES 150-159. 150-151, S. naiquanlini, head, frontal view: 150, female, 151, male. 152156, S. formosanus: 152, head, female, frontal view, 153, head, male, frontal view, 154, head, female, posterior view, 155, male, antenna, part, 156, mesoscutum, dorsal view. 157-159, $S$. symbioticus: 157, head, female, posterior view, 158, antenna, male, part, 159, mesoscutum, dorsal view.

11. Head very delicately coriaceous without striae and deep punctures on frons, vertex and interocellar area; mesoscutum uniformly alutaceous or very delicately coriaceous, without distinct transverse interrupted striae between notauli ......................... S. hakonensis - Head always with rugose sculpture, with distinct deep punctures on frons, vertex and interocellar area, with striae on frons; mesoscutum with strong surface sculpture, coriaceous or rugose, with distinct elevated transverse rugae, especially in between notauli 
12. Female and male head in frontal view trapezoid, height of eye 1.8-1.9x longer than length of malar space; in female POL:OOL:LOL=2.1:1:1.2 (Fig. 148); length of lateral ocellus in male nearly equal to POL; F1 in female 1.3-1.4x longer than F2 S. ishikarii - Female and male head in frontal view rounded or ovate, height of eye only $1.45 \mathrm{x}$ longer than length of malar space; in female POL:OOL:LOL=2.8:1:1.2 (Fig. 149); length of lateral ocellus in male $1.5 \mathrm{x}$ shorter than length of POL; F1 in female nearly equal in length to F2 S. belizinellus 13. Head of female and male in frontal view triangular, higher than broad; genae very narrow, straight aside head in frontal view, never rounded (Figs 150-151) S. xialongmeni - Head of female and males in frontal view always rounded, always broader than high; gena bucate, in some species partially visible behind eye in frontal view (Figs 152-153) 14

14. Head and mesosoma reddish brown S. chinensis

- Head reddish brown, mesosoma always black or very darkish black 15 15. Postgena without setae (Fig. 154); F1 in male curved but not expanded apically (Fig. 155); median mesoscutal line deeply impressed in posterior half, extending to $2 / 3$ of mesoscutum, never complete (Fig. 156); mesoscutellum slightly longer than broad S. formosanus - Postgena with dense white setae especially along hypostomata (Fig. 157); F1 in male curved and expanded apically (Fig. 158); median mesoscutal line strongly impressed, complete, reaches pronotum (Fig. 159); mesoscutellum 1.4× longer than broad

S. symbioticus

\subsubsection{Annotated list of Eastern Palearctic Synergus species}

All Eastern Palearctic species are given below in alphabetical order, with brief data on their biology, hosts and distribution (Schwéger et al. 2015a).

Synergus abei Melika \& Schwéger, 2015

This species was reared from undescribed acorn galls on Q. crispula and Q. serrata (Figs A123, A126) and unknown bud galls (Figs A124-A125) collected from Q. crispula. Adults emerged under laboratory conditions during October. Currently known only from Japan (Hokkaido and Kyushu). Diagnosis and detail species description are given in Schwéger et al. (2015a). 
Synergus belizinellus Schwéger \& Melika, 2015

This species was reared mainly from asexual leaf galls of Belizinella vicina Kovalev (Fig. A119) collected from $Q$. mongolica and also from two morphotypes of undescribed leaf galls (Figs A121-A122) collected from $Q$. dentata and $Q$. crispula. Adults emerged under laboratory conditions in October-November. Currently known only from the Far East Russia, Primorskij Kraj and Japan (Hokkaido). Diagnosis and detail species description are given in Schwéger et al. (2015a).

Synergus brevis (Weld, 1926)

Originally described as Saphonecrus brevis Weld, 1926 based on the open radial cell of the forewing (Weld 1926). Currently known from the USA (New Mexico and Arizona) and was reared from Andricus ruginosus Bassett galls developing on section Quercus of genus Quercus oaks (Weld 1926, Burks et al. 1979). The holotype female, deposited at the USNM, labeled as "Magdalena, N.M.", "Quercus grisea", red "Type 27224 USNM", "Saphonecrus brevis Weld", was examined and the species is transferred to Synergus (Schwéger et al. 2015b). The radial cell of the forewing is partially open, $\mathrm{R} 1$ reaching the wing margin and runs along the margin onto $1 / 3$ of the radial cell length. The frontal carina is strong, complete, and reaches the lateral ocellus; the head is broadened behind the eye in frontal view; the female antenna has 12 flagellomeres. The side of the mesosoma, in dorsal view, is rounded, the lateral pronotal carina is absent; the mesoscutum has strong transverse rugae, the space between the rugae is smooth; the notaulus is complete, reaching the anterior margin of the mesoscutum; the metapleural sulcus is nearly straight, and does not reach the posterior margin of the mesopectus; the tarsal claws are simple; the first metasomal tergite has delicate, indistinct sulci laterally, absent dorsally; the syntergite has a row of white setae anterolaterally, is not incised dorsoposteriorly, and has a narrow band of micropunctures which nearly reaches the ventral edge of the tergite. The combination of characters is more typical for Synergus, thus, we transferred this species to Synergus as Synergus brevis (Weld, 1926) (Schwéger et al. 2015a).

Synergus changtitangi Melika \& Schwéger, 2015

This species was reared from undescribed multilocular stem swelling-like galls (Fig. A127) collected from $Q$. serrata. Adults emerged during March. Currently known from Taiwan (Nantou County). Diagnosis and detail species description are given in Schwéger et al. (2015a). 
Synergus chinensis Melika, Ács \& Bechtold, 2014

This species was described from north-eastern China (Beijing Province), reared from unknown cynipid acorn, leaf and bud galls (Melika et al. 2004). Later it was found in South Korea (Abe et al. 2007). Kovalev (1965) mentioned S. gallaepomiformis (Boyer de Fonscolombe) for the Far East of Russia, which was reared from asexual galls of Andricus hakonensis (= A. symbioticus, $=$ A. attractus). We strongly doubt this identification. On the basis of the adult description given by Kovalev, these specimens belong to $S$. chinensis (Melika et al. 2004) which falls into a subclade with $S$. symbioticus and can be easily distinguished from the latter by some bright diagnostic characters given in the key to Synergus. Currently known from China, South Korea and Russia.

Synergus formosanus Schwéger \& Melika, 2015

This species was reared only from the asexual galls of Trichagalma formosana (Fig. A118) on Q. variabilis, adults emerge under laboratory conditions in October. Currently known from Taiwan (Nantou County). Diagnosis and detail species description are given in Schwéger et al. (2015a).

Synergus gifuensis Ashmead, 1904

Synergus gifuensis was described from three specimens (Ashmead 1904). The type female labelled as "Y, Nawa, Gifu, Jap. det. 1902", red label "Female Type No.7304 USNM", Ashmead's handwritten label "Synergus gifuensis" was examined by us. Abe (1990) referred to this species as Synergus japonicus type B. which appeared to be univoltine and arrhenotokous. Adults emerge from asexual galls of Andricus mukaigawae in early June. Galls of $A$. mukaigawae inhabited by S. gifuensis are usually larger than those inhabited by S. japonicus, and the inquiline larval cells are usually separated by a woody wall inside the host gall (Abe 1990, 1992a, Pujade-Villar et al. 2002). A detailed description of the adults, species diagnosis and additional data on hosts (asexual galls of A. kashiwaphilus), was given by Pujade-Villar et al. (2014b). Wasps were reared from galls of A. mukaigawae, collected in Japan (Hokkaido) and the Far East Russia (Primorskij Kraj, Khazan Lake).

Synergus hakonensis Ashmead, 1904

Synergus hakonensis is a valid species, most closely resembles $S$. ishikarii and $S$. belizinellus by the rounded head in frontal view. In $S$. hakonensis the head is very delicately coriaceous, without 
striae and deep punctures on the frons, vertex and interocellar area; the mesoscutum is uniformly alutaceous or very delicately coriaceous, without distinct transverse interrupted striae between notauli, while in $S$. ishikarii and $S$. belizinellus the head is always with rugose sculpture, with distinct deep punctures on the frons, vertex and interocellar area, with striae on the frons; the mesoscutum with strong surface sculpture, coriaceous or rugose, with distinct elevated transverse rugae, especially in between notauli (for more diagnostic characters see also the key to species). Currently the species is known only from the one type specimen collected in Japan (Schwéger et al. 2015a).

Synergus hupingshanensis (Liu, Yang \& Zhu, 2012)

In this species, the female antenna has 12 flagellomeres, the tarsal claws have a triangular basal lobe; and the frontal carina is weak, complete, and reaches the torulus. The radial cell of the fore wing is partially open, with R1 reaching the wing margin and running a short distance along the margin. It is known from China (Guanshan). The examination of the detailed description and illustrations of this species showed that it is not a Saphonecrus but a Synergus species: Synergus hupingshanensis (Liu, Yang \& Zhu) which belongs to the group of Synergus species (Synergus castaneus, S. plagiotrochi and S. kawakamii) with partially open radial cell of the fore wing (Schwéger et al. 2015a). This species is associated with galls developing on Castanopsis carlesii which from Liu et al. (2012) never reared any gallwasp, thus they do not exclude the possibility that S. hupingshanensis might be a gall inducer (Liu et al. 2012). Such is the case with Synergus itoensis Abe, Ide \& Wachi (Abe et al. 2011).

Synergus ishikarii Melika \& Schwéger, 2015

This species was reared from asexual leaf galls of Ussuraspis sp. (Fig. A120) and undescribed leaf galls (Fig. A121) on Q. dentata and unknown leaf galls (Fig. A122) collected from $Q$. crispula. Adults emerged under laboratory conditions in October-November. Currently known from Japan (Hokkaido). Diagnosis and detailed species description are given in Schwéger et al. (2015a).

\section{Synergus iwatensis Shinji, 1941}

Japan. The type lost, status is uncertain (Abe et al. 2007). 
Synergus japonicus Walker, 1874

Detailed re-description, diagnosis, and data on biology and distribution were provided by Pujade-Villar et al. (2014b). The biology of the species was described in details in Abe (1990, 1992a). For a long time the species was known only from Japan, but recently also recorded from China (Pujade-Villar et al. 2014b). We collected and reared S. japonicus in large numbers from galls of A. kashiwaphilus in the Far East Russia (Primorskij Kraj, Khazan Lake). Currently the species is known from Japan, China and Russia (Schwéger et al. 2015a).

Synergus jezoensis Uchida \& Sakagami, 1948

This species was recently re-validated, types were examined and a detail re-description with diagnosis was provided by Pujade-Villar et al. (2014b). Species is known from Japan and China (Zhejiang); biology is unknown (Pujade-Villar et al. 2014b).

Synergus kawakamii Tang \& Melika, 2015

This species was reared from undescribed irregular spheric galls on Castanopsis kawakamii. Currently known from Taiwan (Hualien County). It is the first Synergus species reared from galls on Castanopsis. Diagnosis and detail species description are given in Schwéger et al. (2015a).

Synergus khazani Melika \& Schwéger, 2015

Most specimens were reared from the asexual galls of $A$. kashiwaphilus (Fig. A115) and $A$. mukaigawae (Fig. A116), collected from Q. mongolica and Q. dentata; few specimens were obtained from an unknown bud gall (Fig. A124) and unknown acorn gall (Fig. A126), both collected from Q. crispula. Adults emerge under laboratory conditions in October-November. Currently known from the Far East Russia (Primorskij Kraj, Khazan Lake and Vityaz Bay), Japan (Hokkaido) (Schwéger et al. 2015a) and China (Pujade-Villar et al. 2016). Diagnosis and detail species description are given in Schwéger et al. (2015a).

\section{Synergus mizunarae Shinji, 1940}

Japan. The type is lost; status is uncertain (Abe et al. 2007). 
Synergus symbioticus Schwéger \& Melika, 2015

This species was reared from the asexual galls of Andricus hakonensis (= A. symbioticus) (Fig. A117) and A. kashiwaphilus on $Q$. dentata and Q. mongolica subsp. crispula. Adults emerge in October. Currently known from Japan (Hokkaido) and the Far East Russia (vicinity of Vladivostok). Diagnosis and detail species description are given in Schwéger et al. (2015a).

Synergus xialongmeni Melika, Ács \& Bechtold, 2004

Currently known from China (Beijing Province), reared from unknown cynipid acorn, leaf and bud galls (Melika et al. 2004). This species shows some morphological similarity to $S$. belizinellus and S. ishikarii.

Synergus yukawai (Wachi, Ide \& Abe, 2011)

This species known from Japan (Honshu and Kyushu) was reared from a gall midge Ametrodiplosis acutissima (Monzen) (Diptera: Cecidomyiidae) galls on Q. acutissima (Quercus section Cerris) (Wachi et al. 2011b). The species has a strong, complete lateral frontal carina, and thus, it is not a Saphonecrus and was transferred by us to Synergus. The species belongs to the group of Synergus species with partially open radial cell of the fore wing, such as Synergus castaneus, S. plagiotrochi and S. kawakamii (Schwéger et al. 2015a).

\subsection{Phylogenetics of Synergini sensu stricto}

Sixty taxa were included in the phylogenetic reconstruction (Table 8) of Palearctic species of Synergini sensu stricto (Pénzes et al. 2012). The phylogenetic analysis is based on 1,240 aligned sequence positions. The phylogenetic position of Synophrus, Lithosaphonecrus, Saphonecrus and Ufo has been recently re-appraised and established (Bozsó et al. 2014; Melika et al. 2005, 2007, 2012; Pénzes et al. 2009, 2012; Nieves-Aldrey \& Butterill 2014). Bozsó et al. (2014) included sequences from the newly described Saphonecrus species, too. Similarly to the previous studies, Lithosaphonecrus, Ufo, Synergus and Synophrus were recovered as monophyletic groups (genera) in contrast to Saphonecrus (Fig. 160).

Four basal clades were established (Pénzes et al. 2012, Bozsó et al. 2014), Synophrus, Saphonecrus \#1, Saphonecrus-\#2 and a large clade with all the remaining lineages, although 
their relationships are generally unresolved. The latter large clade is divided into several lineages, including Ufo, Lithosaphonecrus, five Saphonecrus lineages from EP and WP, and Synergus.

Table 8. GenBank accession number of haplotype sequences used in the phylogenetic reconstructions. Names between quotation marks refer to the labels used in the previous referred studies. Note that samples S27 and S57 appeared as Saphonecrus shirokashicola in the earlier literature and corrected in Schwéger et al. $(2015 \mathrm{a}, \mathrm{b})$.

\begin{tabular}{|c|c|c|c|}
\hline Lineage & cox1 haplotype & 28S D2 haplotype & References \\
\hline Lithosaphonecrus dakengi & KC899797 & KC899801 & Bozsó et al. 2015 \\
\hline Lithosaphonecrus formosanus & KC899798 & KC899802 & Bozsó et al. 2015 \\
\hline Lithosaphonecrus huisuni & KC899795 & KC899799 & Bozsó et al. 2015 \\
\hline Lithosaphonecrus yunnani & KC899796 & KC899800 & Bozsó et al. 2015 \\
\hline Rhoophilus loewi (outgroup) & EF486876 & EF487123 & Ács et al. 2010 \\
\hline Saphonecrus barbotini & EF486877 & EF487124 & Ács et al. 2010 \\
\hline Saphonecrus chinensis "S30" & KF532109 & KF532097 & Bozsó et al. 2014 \\
\hline Saphonecrus connatus & EF486878 & EF487125 & Ács et al. 2010 \\
\hline Saphonecrus gallaepomiformis & EF486881 & EF487131 & Ács et al. 2010 \\
\hline Saphonecrus gilvus "TWTl12" & JX468364 & JX468369 & Melika et al. 2012 \\
\hline Saphonecrus globosus "S18" & KF532107 & KF532094 & Bozsó et al. 2014 \\
\hline Saphonecrus haimi & EF486879 & EF487126 & Ács et al. 2010 \\
\hline Saphonecrus lithocarpii "S32" & KF532110 & KF532098 & Bozsó et al. 2014 \\
\hline Saphonecrus longinuxi"S 2,JP02" & JX468362 & JX468368 & Melika et al. 2012 \\
\hline Saphonecrus longinuxi "S9, JP02" & JX468363 & JX468368 & Melika et al. 2012 \\
\hline Saphonecrus morii "S11" & KF532105 & KF532092 & Bozsó et al. 2014 \\
\hline Saphonecrus morii "S49" & KF532116 & KF532092 & Bozsó et al. 2014 \\
\hline Saphonecrus nantoui "S23" & KF532108 & KF532095 & Bozsó et al. 2014 \\
\hline Saphonecrus nantoui "S35” & KF532112 & KF532095 & Bozsó et al. 2014 \\
\hline Saphonecrus nichollsi "S36" & KF532113 & KF532100 & Bozsó et al. 2014 \\
\hline Saphonecrus pachylomai "S48" & KF532115 & KF532102 & Bozsó et al. 2014 \\
\hline Saphonecrus saliciniai" S57" & KF532122 & JX468371 & Bozsó et al. 2014 \\
\hline Saphonecrus saliciniai "S27" & KF532121 & KF532091 & Bozsó et al. 2014 \\
\hline Saphonecrus shanzhukui "S15" & KF532106 & KF532093 & Bozsó et al. 2014 \\
\hline Saphonecrus shanzhukui "S46" & KF532114 & KF532101 & Bozsó et al. 2014 \\
\hline Saphonecrus shirakashii & JX468365 & JX468370 & Melika et al. 2012 \\
\hline Saphonecrus shirokashicola & JX468366 & JX468371 & $\begin{array}{l}\text { Bozsó et al. 2014; } \\
\text { Melika et al. } 2012\end{array}$ \\
\hline Saphonecrus symbioticus "S50" & KF532117 & KF532103 & Bozsó et al. 2014 \\
\hline Saphonecrus symbioticus "S51" & KF532118 & KF532103 & Bozsó et al. 2014 \\
\hline Saphonecrus taitungi "S34" & KF532111 & KF532099 & Bozsó et al. 2014 \\
\hline Saphonecrus undulatus & EF486883 & EF487133 & Ács et al. 2010 \\
\hline Synergus abei & KR270551 & KR270534 & Schwéger et al. $2015 \mathrm{a}$ \\
\hline Synergus acsi & EF486884 & EF487134 & Ács et al. 2010 \\
\hline Synergus belizinellus & KR270555 & KR270536 & Schwéger et al. 2015a \\
\hline Synergus castaneus & KC533850 & KC533844 & Bernardo et al. 2013 \\
\hline
\end{tabular}




\begin{tabular}{|c|c|c|c|}
\hline Lineage & cox1 haplotype & 28S D2 haplotype & References \\
\hline Synergus chinensis & EF486890 & EF487140 & Ács et al. 2010 \\
\hline Synergus consobrinus & EF486955 & EF487190 & Ács et al. 2010 \\
\hline Synergus crassicornis & EF486898 & EF487147 & Ács et al. 2010 \\
\hline Synergus incrassatus & EF486925 & EF487165 & Ács et al. 2010 \\
\hline Synergus japonicus & KR270560 & EF487167 & Schwéger et al. $2015 \mathrm{a}$ \\
\hline Synergus formosanus & KR270545 & KR270532 & Schwéger et al. $2015 \mathrm{a}$ \\
\hline Synergus ishikarii & KR270548 & KR270533 & Schwéger et al. 2015a \\
\hline Synergus khazani & KR270557 & KR270537 & Schwéger et al. $2015 \mathrm{a}$ \\
\hline Synergus mikoi & EF486928 & EF487169 & Ács et al. 2010 \\
\hline Synergus plagiotrochi & EF486952 & EF487188 & Ács et al. 2010 \\
\hline Synergus symbioticus "S7" & KR270540 & KR270530 & Schwéger et al. $2015 \mathrm{a}$ \\
\hline Synergus symbioticus "S122" & KR270541 & KR270530 & Schwéger et al. $2015 \mathrm{a}$ \\
\hline Synergus thaumacerus & EF486957 & EF487222 & Ács et al. 2010 \\
\hline Synergus xiaolongmeni & EF486968 & EF487220 & Ács et al. 2010 \\
\hline Synergus sp "MOTU2 S110" & HM574230 & HM574142 & Kaartinen et al. 2010 \\
\hline Synergus sp "MOTU4 S30" & HM574169 & HM574132 & Kaartinen et al. 2010 \\
\hline Synergus sp. "SP10 flavipes" & EF486903 & EF487151 & Ács et al. 2010 \\
\hline Synophrus olivieri & EF579725 & EF583959 & $\begin{array}{c}\text { Ács et al. 2010; } \\
\text { Pénzes et al. } 2009\end{array}$ \\
\hline Synophrus pilulae & EF579716 & EF487224 & $\begin{array}{l}\text { Ács et al. 2010; } \\
\text { Pénzes et al. } 2009\end{array}$ \\
\hline Synophrus politus & EF579710 & EF487223 & $\begin{array}{c}\text { Ács et al. 2010; } \\
\text { Pénzes et al. } 2009\end{array}$ \\
\hline Ufo cerroneuroteri" S8" & JX468357 & JX468367 & Melika et al. 2012 \\
\hline Ufo cerroneuroteri" S14" & JX468358 & JX468367 & Melika et al. 2012 \\
\hline Ufo nipponicus "S38" & JX468359 & JX468367 & Melika et al. 2012 \\
\hline Ufo nipponicus "S39" & JX468360 & JX468367 & Melika et al. 2012 \\
\hline Ufo nipponicus "S40" & JX468361 & JX468367 & Melika et al. 2012 \\
\hline
\end{tabular}




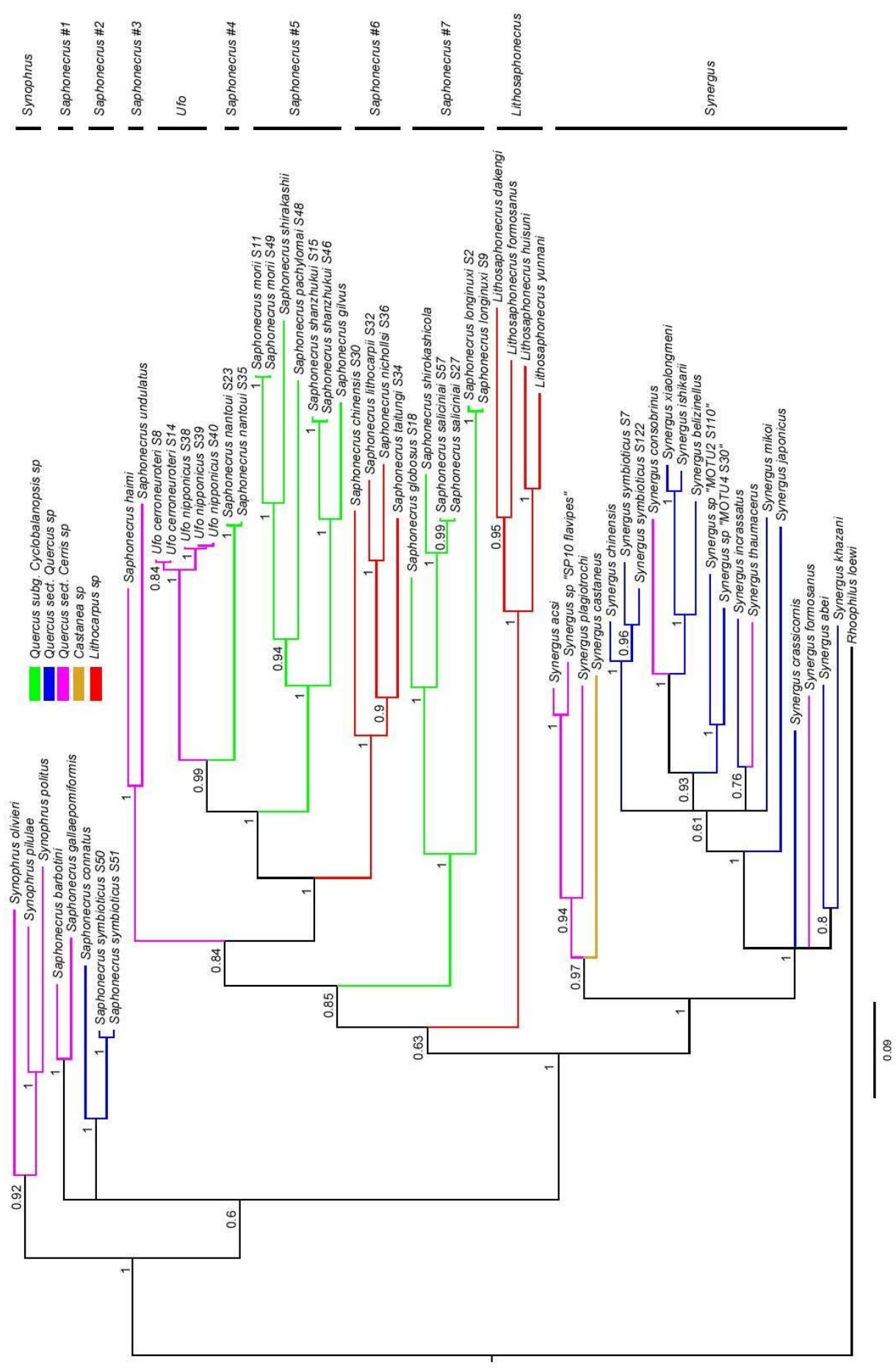

FIGURE 160. Bayesian majority rule consensus phylogeny of Saphonecrus and Synergus. Host plant associations are coded by colors. Numbers at nodes indicate estimated posteriori clade probabilities (Schwéger et al. 2015a, b). 
The division of the genus Synergus into section I and section II (Mayr 1872) appeared to be artificial, with each section being polyphyletic (Ács et al. 2010). Our phylogenetic tree support this conclusion and the newly described species of Synergus from the EP span the Synergus phylogenetic tree: species allocated morphologically to Section I (S. abei, S. changtitangi, S. khazani) are intermixed with those allocated to Section II (S. belizinellus, S. formosanus, S. ishikarii S. symbioticus) (Fig. 160).

Concerning the monophyly of Synergus, it is important to state that only WP and EP Synergus species were included in the analyses, so the worldwide monophyly of Synergus remains to be tested. Although the reconstruction included a subset of Synergus examined in detail, Palearctic Synergus remains monophyletic (Schwéger et al. 2015a). The eight newly described EP Synergus species fall into 6 distinct clades, although sistergroup relationships remain uncertain in many cases (Fig. 160). Synergus belizinellus, S. ishikarii, and S. xialongmeni fall into one subclade. These species also form a distinct morpho-group, and characterized by a rugose head, the frons and vertex are with deep punctures and striae, the mesoscutum with strong surface sculpture. Another distinct subclade, Synergus chinensis and S. symbioticus, also possess distinct set of morphological character states: the head of the female and male in frontal view always is rounded and broader than high; the gena is bucate and partially visible behind eye in frontal view. On the other hand, S. japonicus and S. khazani, which are falling into different subclades, are showing similarity in some morpho-characters: the dull rugose mesoscutum, deep scutellar foveae, rugose frons and vertex, strong carinae on the lower face. Grouping of EP Synergus species into different subclades (Fig. 160) not necessarily followed by a distinct set of morpho-characters. Host associations are interpreted in Discussion.

\subsection{Eastern Palearctic Cynipini}

\subsubsection{Eastern Palearctic oak gallwasps}

Herein listed 85 EP species of Cynipini trophically associated with ten Quercus subgenus Quercus species, 9 Quercus subgenus Cyclobalanopsis, 2 Castanopsis, 6 Castanea and 1 Lithocarpus species (unpublished results). The oak gallwasp faunas of WP, EP and Nearctic are very peculiar and each biogeographical region possesses with its own distinct species diversity without any overlap. The number of known Cynipini species in the WP is 2 times higher than the number of species described from the EP. However, the EP fauna is much less studied and 
further research definitely will reveal a great number of new species. It is expected that the host diversity will also increases with further research. Currently many more gall morphotypes are known which are differ from the described species and might well represent new, undescribed species. The annotated list of EP Cynipini with including species known from the Oriental Region is given in Appendix 9.2., while the list of species with uncertain status is given in Appendix 9.3 (unpublished results).

The number of recognised species in each genus of Cynipini in WP and EP are given in Table 9.

Table 9. Comparison of Western and Eastern Palearctic Cynipini oak gallwasp fauna

\begin{tabular}{|l|c|c|}
\hline Genus & WP & EP \\
\hline Andricus Hartig, 1840 & 99 & 16 \\
\hline Aphelonyx Mayr, 1881 & 3 & - \\
\hline Belizinella Kovalev, 1965 & - & 2 \\
\hline Biorhiza Westwood, 1840 & 1 & 1 \\
\hline Callirhytis Foerster, 1869 & 6 & 1 \\
\hline Cerroneuroterus Melika \& Pujade-Villar, 2009 & 6 & 3 \\
\hline Chilaspis Mayr, 1881 & 2 & - \\
\hline Cyclocynips Melika, Tang, \&Sinclair, 2013 & - & 2 \\
\hline Cycloneuroterus Melika \& Tang, 2011 & - & 17 \\
\hline Cynips Linnaeus, 1758 & 9 & 1 \\
\hline Dryocosmus Giraud, 1859 & 6 & 12 \\
\hline Latuspina Monzen, 1954 & - & 9 \\
\hline Neuroterus Hartig, 1840 & 6 & 7 \\
\hline Plagiotrochus Mayr, 1881 & 14 & 7 \\
\hline Pseudoneuroterus Kinsey, 1923 & 4 & - \\
\hline Trichagalma Mayr, 1907 & - & 3 \\
\hline Trigonaspis Hartig, 1840 & 5 & 3 \\
\hline Ussuraspis Kovalev, 1965 & - & 1 \\
\hline TOTAL & $\mathbf{1 6 5}$ \\
\hline
\end{tabular}




\section{DISCUSSION}

\subsection{Integrative taxonomy and phylogeny of Saphonecrus and Synergus species}

Based on combined analyses of morphological, molecular and biological data, Ronquist et al. (2015) rejected the monophyly of inquiline gallwasps (Synergini). Tribe Diastrophini was established for two inquiline genus (Synophromorpha and Periclistus) and Rosaceae gallers with strong phylogenetic support. The Fagaceae host plant and overwhelmingly Cynipini gall associated inquilines (Synergini sensu lato) were divided into two distinct lineages as follows: Synergini sensu stricto including Lithosaphonecrus, Saphonecrus, Synergus, Synophrus and Ufo genera (Synergini sensu stricto) and Ceroptresini (one genus, Ceroptres) with strong support (Ronquist et al. 2015).

Within the tribe Synergini there is some contradiction between the morphology-based taxonomy and the molecular phylogeny when considering the classification above the species level, especially striking in the largest genera, Synergus and Saphonecrus (Melika 2006, Pénzes et al. 2009, Ács et al. 2010).

All of the recently described new EP Saphonecrus and Synergus species are supported by distinct mitochondrial DNA haplotypes, which support the description of novel species established by the morphology. On the other hand, there is some contradiction between morphology-based taxonomy and molecular phylogeny considering the classification above the species level (Schwéger et al. 2015a, b).

Although the presented phylogenetic tree (Fig. 106) is ambiguous in many details, it suggests clearly distinct groups of species with high support. However, in some cases, different clades include species with the same group of diagnostic characters, while other clades include species with different characteristics which can be found in species which belong to different clades. For instance in case of Saphonecrus shirakashii and Saphonecrus shirokashicola, which have a slightly different morphological character states, belong to two different distantly related lineages within Synergini (Figs 118-119, 125-126, 160). Some previously described EP species appear to be erroneously included in Saphonecrus and were moved to Synergus. Besides these inconsequences, all of the described new EP Saphonecrus species (Schwéger et al. 2015b) are characterized by distinct mitochondrial coxI DNA haplotypes (barcodes), which is concordant to

the discrimination of novel species established by the morphology. More reliable estimation of evolutionary relationships and corresponding taxonomy requires further research. Owing to these 
limitations, we decided to leave all the newly described species within Saphonecrus because it is too early to establish new genera until the basic divisions within Saphonecrus are solved (Schwéger et al. 2015b).

The genus Lithosaphonecrus Tang, Melika \& Bozsó, with four species, which most closely related to Saphonecrus and forms a distinct lineage within Synergini, was described from China and Taiwan (Bozsó et al. 2015, online version in 2013). Lithonecrus Nieves-Aldrey \& Butterill, with one species L. papuanus Nieves-Aldrey \& Butterill, collected from Lithocarpus celebicus, was described from Papua New Guinea (Nieves-Aldrey \& Butterill 2014), was synonymized to Lithosaphonecrus (Schwéger et al. 2015a), as Lithosaphonecrus papuanus (Nieves-Aldrey \& Butterill, 2014). The known geographic distribution of Lithosaphonecrus is expanded in this way.

\subsection{Phylogenetics of Synergini sensu stricto}

The phylogenetic position of Lithosaphonecrus, Saphonecrus, Synophrus and Ufo has recently been re-appraised and re-established with a more detailed taxon sampling (Bozsó et al. 2014, 2015, Melika et al. 2005, 2007, 2012; Pénzes et al. 2012).

The current phylogenetic and morphological examination of the EP Synergus supports Ács et al. (2010)'s conclusion that palearctic Synergus comprise a monophyletic group, with previously known and here described EP species nested among the WP species. There is thus no evidence that EP and WP Synergus species represent independent regional radiations. However, the monophyly of worldwide Synergus must be treated carefully. Many species assigned to Synergus in the Nearctic show significant morphological differences from palearctic taxa (Ács et al. 2010, Pénzes et al. 2012, Melika et al. 2012, Bozsó et al. 2014, 2015). Preliminary unpublished analysis shows at least three distinct groups within Nearctic Synergus, and assessment of their status, and of the genus as a whole, requires a new phylogenetic re-analysis (unpublished results).

The monophyletic Synergus is nested within Saphonecrus as well as the Ufo and Lithosaphonecrus genera (Fig. 160) (Melika et al. 2012, Bozsó et al. 2014, 2015), according to the two gene sequence based molecular phylogeny. That is, Saphonecrus does not seem to be monophyletic. Molecular phylogenetic analyses of Palearctic Synergini (Pénzes et al. 2012, Bozsó et al. 2014, Bozsó 2015, Schwéger et al. 2015a, b; Fig. 160) suggested three basal 
lineages, although their sister-group relationships are unresolved. Two of these clades include Saphonecrus species described recently (Schwéger et al. 2015a). "Saphonecrus \#2”, with S. connatus and S. symbioticus, seem to represent independent divergences from all others that share a common ancestor (Fig. 160). This lineage is associated with the white oaks section of Quercus. All the molecular data, together with some morphological peculiarities, suggests that it is clearly a distinct unit, different from the "barbotini-gallaepomiformis" ("Saphonecrus \#1", Pénzes et al. 2009, Ács et al. 2010) associated with section Cerris and other Saphonecrus clades discussed below.

According to the host plant associations, the third clade (Fig. 160) may represent a large eastern radiation and can be divided into several further lineages. Some of these include Saphonecrus species exclusively (Saphonecrus \#3-\#7, Fig. 160, Bozsó et al. 2014). Saphonecrus haimi and S. undulatus (Saphonecrus \#3), known from Quercus section Cerris hosts, may be the unique western representative of the eastern radiation on different host plant lineages (Bozsó et al. 2014). On the basis of molecular evidence and also morphological peculiar characters, the "undulatus-haimi" lineage is very distant from the western "barbotinigallaepomiformis" (Saphonecrus \#1) and “connatus" (Saphonecrus \#2) clades (Bozsó et al. 2014).

Our phylogeny may suggest a radiation on Lithocarpus hosts (Saphonecrus \#6). Furthermore, the Cerris section specific $U f o$ belong to a well-supported clade with members associated with Quercus subgenus Cyclobalanopsis exclusively (Saphonecrus \#4-\#5). This clade includes Saphonecrus shirakashii and five newly described species. There is another clade on Quercus subgenus Cyclobalanopsis hosts (Saphonecrus \#7), that includes Saphonecrus shirokashicola and three new species. However, owing to the limitations of our reconstruction, it is not clear at present whether these host-specific lineages represent independent radiations (Bozsó et al. 2014, 2015).

Of the 27 Saphonecrus species for which the host gall and host plant associations are known, represented partly in the phylogenetic analysis, 11 species associate with hosts developing on Quercus subgenus Quercus, 12 species with hosts developing on Quercus subgenus Cyclobalanopsis, and 4 species associate with Lithocarpus (Table 2). The majority of Eastern Palearctic Saphonecrus species associate with galls developing on Quercus subgenus Cyclobalanopsis and Lithocarpus species, form distinct subclades (Bozsó et al. 2014, 2015). One 
species, Saphonecrus hupingshanensis Liu, Yang \& Zhu, was known to associate with galls developing on Castanopsis (Liu et al. 2012), however, based on the morphological examination, it was transferred herein to Synergus, thus currently no Saphonecrus species are known to associate with the Castanea-Castanopsis lineage of Fagaceae.

Of 14 Eastern Palearctic Synergus species for which the host gall and host plant associations are known, 11 species associated with hosts developing on Quercus subgenus Quercus (Table 2). One species, S. itoensis is a gall-inducer on acorns of Quercus subgenus Cyclobalanopsis. Two Synergus species with opened or partially opened radial cell of the fore wing, S. kawakamii and S. castaneus, could not be placed reliably on the phylogenetic tree. None-Synergus cynipid inquilines are known to associate with oak galls (Cynipini) developing on Castanopsis and Lithocarpus. Saphonecrus excisus (Kieffer), described from Bengal, Lithosaphonecrus species from China, Taiwan and Papua New Guinea are known to associate with Lithocarpus (Bozsó et al. 2013, Nieves-Aldrey \& Butterill 2014). In spite of the recent discovery of a rich oak gallwasp fauna associated with non-Quercus Fagaceae, with the exceptions of $S$. itoensis (on Cyclobalanopsis) and S. kawakamii (Castanopsis) no other Synergus species are known to be associated with galls developing on Quercus subgenus Cyclobalanopsis, Castanopsis and Lithocarpus. On the other hand, very few Saphonecrus, Lithosaphonecrus and Saphonecrus-like species have been reared from galls of Andricus, Cerroneuroterus, Latuspina, Plagiotrochus, and Trichagalma genera of Cynipini that associate with Quercus subgenus Quercus species - in galls induced by these Cynipini, only Synergus and Ufo species have so far been found.

Futher research is needed to decide whether the Synergus and Saphonecrus-like inquilines have preferences in host gall and associate host plant and if so, whether such preferences reflect fundamental evolutionary trends in host tracking or codiversification, as observed for the Cynipini gallwasps (Cook et al. 2002, Stone et al. 2009). A deep evolutionary split was supposed to be present in host plant associations of inquilines, particularly those of the Synergus-complex (Ács et al. 2010). However, recent studies suggest examples for different independent radiations on the same host lineages (Bozsó et al. 2014). The most striking example is provided by the two Saphonecrus lineages present on Lithocarpus, one of which was described as a new genus, Lithosaphonecrus (Bozsó et al. 2015, online in 2013]). The early split within the Fagaceae between Quercus and Lithocarpus (Oh \& Manos 2008) is not reflected in the inquiline 
phylogeny. Furthermore, the section Cerris-specific, eastern Ufo and the western "undulatushaimi" clade seem to be embedded in the eastern clade, characteristic to the hosts from subgenus Cyclobalanopsis of Quercus and Lithocarpus, all known from the EP. Further research is needed to clarify host shifting events in cynipid inquilines.

\subsection{Eastern Palearctic oak gall inducers}

Oak gallwasps (Hymenoptera: Cynipidae: Cynipini) are by far the most species-rich group of gallwasps (Csóka et al. 2005, Ronquist et al. 2015; Table 1). Although the EP fauna is less known, it has probably large species richness.

From 85 EP Cynipini species about the half (49 species) associate with Quercus subgenus Quercus, while 25 species with Quercus subgenus Cyclobalanopsis and only eight species known to associate with the rest three oak related Fagaceae genera, Castanea, Castanopsis and Lithocarpus (Abe et al. 2014a, b, Ide et al. 2010, 2012, 2013, 2016, Melika et al. 2011, 2013, Tang et al. 2011a, b).

Of the ten described EP species of Dryocosmus two species (D. sakureiensis Ide, Wachi \& Abe, D. sefuriensis Ide, Wachi \& Abe) are associated with Cyclobalanopsis, two further species (D. nanlingensis Abe, Ide, \& Odagiri, D. okajimai Abe, Ide, Konishi \& Ueno) are probably associated with Cyclobalanopsis, four species (D. carlesiae Tang \& Melika, D. pentagonalis Melika \& Tang, D. testisimilis Tang \& Melika, D. triangularis Melika \& Tang) are associated with Castanopsis, and two species (D. kuriphilus (Yasumatsu), and D. zhuili Liu \& Zhu) are associated with Castanea (Abe et al. 2014a, b, Ide et al. 2010, 2012, 2013, Melika et al. 2011, 2013, Tang et al. 2011a, b, Zhu et al. 2015). Other 12 new Dryocosmus species are under description which from five species associates with Castanopsis and other seven species with Cyclobalanopsis. The genus Dryocosmus with its current classification and limits undoubtedly forms a polyphyletic group, as is discussed in detail by Melika et al. (2011). Palearctic Dryocosmus contains at least three major groups - kuriphilus and other East Asian species, and two WP groups consisting of mayri/caspiensis and related taxa, and cerriphilus. The morphological and genetic diversity of East Asian Dryocosmus would also imply that Asian members of this genus may reperesent multiple distinct lineages. Hence, further phylogenetic analysis involving all known Dryocosmus species is necessary to redefine "true" Dryocosmus, 
alongside detailed examination of morphological character states in each lineage (Melika et al. 2011).

Most oak gallwasps are cyclically parthenogenetic, with obligate alternation between a sexual generation involving males and females that usually occurs at the same time as the flush of new growth on host plants in spring, and the asexual generation with only parthenogenetic females occurring in winter (Stone et al. 2002). A proportion of gallwasp taxa are only known from one of these two generations, although a recent study confirmed that many species known only from an asexual generation do also possess a sexual generation (Stone et al. 2008). In all cases where the sexual and asexual generations are known for species within the allied Asian genus Cycloneuroterus, both are induced on the same host plant lineage (various Cyclobalanopsis species; Tang et al. 2011a, 2016a), as is typical of most other oak gallwasps. Of the 12 described Eastern Palearctic Dryocosmus species only one species, D. kunugiphagus, is known yet to have alternate sexual and asexual generations (Ide \& Abe 2015). Hence further sampling on the appropriate host plants should reveal the alternate generations of other Asian Dryocosmus species and understand the peculiar pattern of alternate generations. 


\section{ACKNOWLEDGEMENTS}

Firstly, I would like to express my sincere gratitude to my supervisors Dr. Pénzes Zsolt and Dr. Melika George for the continuous support of my Ph.D study and related research, for their patience, motivation, and immense knowledge. Their guidance helped me in all the time of research and writing of this thesis.

I would like to thank my colleagues from Department of Ecology, University of Szeged for their wonderful collaboration and countless happy moments. They supported me greatly and were always willing to help me. Also I am grateful to my colleagues of the Plant Health and Molecular Biology Laboratory, National Food Chain Safety Office for all the good memories we have had in the half year experience.

I thank my fellow labmates: Dr. Bihari Péter and Dr. Bozsó Miklós in Molecular Diversity Laboratory, Biological Research Center of Szeged for the stimulating discussions, for the busy days we were working together and for the helpful knowledge in my molecular research.

I thank Mr. Chang-Ti Tang (National Chung Hsing University, Taichung, Taiwan) and Dr. György Csóka (NARIC, Forest Research Institute, Department of Forest Protection, Mátrafüred, Hungary) for their kind permission to use in my work gall images which were taken by them in the field.

My sincere thanks also goes to Dr. Andrew Robert Deans and Dr. István Mikó (Pennsylvania State University, Department of Entomology) for offering me the internship opportunities in their group and leading me working on diverse exciting projects.

Last but not the least, I would like to thank my family and my friends for supporting me spiritually throughout writing this thesis and my life in general. 


\section{REFERENCES}

Abe, Y. (1986) Taxonomic status of the Andricus mukaigawae complex and its speciation with geographical parthogenesis (Hymenoptera: Cynipidae). Applied Entomology and Zoology, 21, 436-447.

Abe, Y. (1988) Trophobiosis between the gall wasp, Andricus symbioticus, and the gallattending ant, Lasius niger. Applied Entomology and Zoology, 23, 41-44.

Abe, Y. (1990) Life cycles of two species of the Synergus japonicus complex (Hymenoptera: Cynipidae). Akitu, 120, 1-7.

Abe, Y. (1991) Host race formation in the gall wasp Andricus mukaigawae. Entomologia Experimentalis et Applicata, 58, 15-20.

Abe, Y. (1992a) Ethological isolation between inquiline gall wasps, the Synergus japonicus complex (Hymenoptera: Cynipidae), which sometimes coexist in host galls. Applied Entomology and Zoology, 27, 527-531.

Abe, Y. (1992b) The advantage of attending ants and gall aggregation for the gall wasp Andricus symbioticus (Hymenoptera: Cynipidae). Oecologia, 89, 166-167.

Abe, Y. (1998) Karyotype differences and speciation in the gall wasp Andricus mukaigawae (s. lat.) (Hymenoptera: Cynipidae), with description of the new species A. kashiwaphilus. Entomologica Skandinavica, 29, 131-135.

Abe, Y. (2006) Taxonomic status of the genus Trichagalma (Hymenoptera: Cynipidae), with description of the bisexual generation. Pp. 288-295. In: Ozaki, K., Yukawa, J., Ohgushi, T. \& Price, P.W. (eds) Galling arthropods and their associates. Springer, Tokyo.

Abe, Y. (2007) Parallelism in secondary loss of sex from a heterogonic life cycle on different host plants in the Andricus mukaigawae complex (Hymenoptera: Cynipidae), with taxonomic notes. Journal of Natural History, 41, 473-480.

Abe, Y., Bhuyan M., Mech, J. Pranab R. Bhattacharyya, P.R., Ide, T., Mishima. M., Suyama, C., Sato, S., Matsuo, K. \& Wachi, N. (2012) Discovery of an oak gall wasp (Hymenoptera: Cynipidae) inducing galls on deciduous oak trees in India. Entomological Science, 15, 340-342.

Abe, Y., Ide, T. \& Odagiri, K.-I. (2014a) Cynipidae (Hymenoptera: Cynipoidea) on Cyclobalanopsis (Fagaceae) in mainland China, with the first record of sexual generation of Cynipini in winter. Annals of the Entomological Society of America, 107, 911-916.

Abe, Y., Ide, T., Konishi, K. \& Ueno, T. (2014b) Discovery of Cynipidae (Hymenoptera: Cynipoidea) From the Indochina Region, With Description of Three New Species. Annals of the Entomological Society of America, 107, 399-406. 
Abe, Y., Ide, T. \& Wachi, N. (2011) Discovery of a new gall-inducing species in the inquiline tribe Synergini (Hymenoptera: Cynipidae): inconsistent implication from biology and morphology. Annals of the Entomological Society of America, 104 (2), 115-120.

Abe, Y., Melika, G. \& Stone, G.N. (2007) The diversity and phylogeography of cynipid gallwasps (Hymenoptera: Cynipidae) of the oriental and eastern palearctic regions, and their associated communities. Oriental Insects, 41, 169-212.

Ács, Z., Challis, R., Bihari, P., Blaxter, M., Hayward, A., Melika, G., Csóka, Gy., Pénzes, Z., Pujade-Villar, J., Nieves-Aldrey, J.L., Schönrogge, K. \& Stone, G.N. (2010) Phylogeny and DNA barcoding of inquiline oak gallwasps (Hymenoptera: Cynipidae) of the Western Palearctic. Molecular Phylogenetics and Evolution, 55, 210-225.

Ács, Z., Melika, G., Pénzes, Z., Pujade-Villar, J. and Stone. G.N. (2007) The phylogenetic relationships between Dryocosmus, Chilaspis and allied genera of oak gallwasps (Hymenoptera, Cynipidae: Cynipini). Systematic Entomology, 32, 70-80.

Aebi, A., Schönrogge, K., Melika, G., Alma, A., Bosio, G., Quacchia, A., Picciau, L., Abe, Y., Moriya, S., Yara, K., Seljak, G. \& Stone, G.N. (2006) Parasitoid recruitment to the globally invasive chestnut gall wasp Dryocosmus kuriphilus. Pp. 103-122. In: Ozaki, K., Yukawa, J., Ohgushi, T. \& Price, P. W.) (eds.) Galling arthropods and their associates; ecology and evolution. Springer, Tokyo. 308pp.

Altekar, G., Dwarkadas, S., Huelsenbeck, J. P. \& Ronquist, F. (2004) Parallel Metropoliscoupled Markov chain Monte Carlo for Bayesian phylogenetic inference. Bioinformatics, 20, 407-415.

Ashmead, W.H. (1885) A Bibliographical and Synonymical Catalogue of the North American Cynipidae, with description of new species. Transactions of the American Entomological Society, 12, 291-304.

Ashmead, W.H. (1896) Descriptions of new cynipidous gall-wasps in the United States National Museum. Proceedings of the U.S. National Museum, 19, 113-136.

Ashmead, W.H. (1904) Description of new Hymenoptera from Japan. (I). Journal of the New York Entomological Society, 12, 65-84.

Askew, R.R. (1984) The Biology of gall wasps. pp. 223-271. In: Ananthakrishnan, T.N. (ed.) Biology of gall insects. Edward Arnold, London.

Belizin, V.I. (1968) New genera and species of gall wasps (Hymenoptera, Cynipoidea) of the Soviet Far East and adjacent territories. Zoologicheskiy Zhurnal, 47 (5), 701-719.

Bellido, D., Ros-Farré, P., Kovalev, O., Pujade-Villar, J. (2000) Presence of Plagiotrochus Mayr, 1881 in the Himalayan area, with redescription of Plagiotrochus semicarpifoliae 
(Cameron, 1902) COMB. N. (Hymenoptera: Cynipidae). Insect Systematics and Evolution, 31, 241-245.

Bernardo, U., Gebiola, M., Xiao, Z., Zhu, C.-D., Pujade-Villar, J. \& Viggiani, G. (2013) Description of Synergus castaneus n. sp. (Hymenoptera: Cynipidae: Synergini) Associated With an Unknown Gall on Castanea spp. (Fagaceae) in China. Annals of the Entomological Society of America, 106 (4), 437-446.

Blaxter, M., Mann, J., Chapman, T., Thomas, F., Whitton, C., Floyd, R. \& Abebe, E. (2005) Defining operational taxonomical units using DNA barcode data. Philosophical Transactions of the Royal Society B., 360, 1935-1943.

Bonfield, J.K., Smith, K.F. \& Staden, R. (1995) A new DNA sequence assembly program. Nucleic Acids Research, 23, 4992-4999.

Bozsó, M. (2015) Taxonomic and phylogenetic studies on the Palearctic Saphonecrus Dalla Torre \& Kieffer oak gall inquiline species (Hymenoptera: Cynipidae, Synergini), Ph.D. Thesis, Szeged Scientific University, Szeged.

Bozsó, M., Pénzes, Z., Bihari, P., Schwéger, S., Tang, C-T., Yang, M.-M., Pujade-Villar, J. \& Melika, G. (2014) Molecular phylogeny of the inquiline cynipid wasp genus Saphonecrus Dalla Torre and Kieffer, 1910 (Hymenoptera: Cynipidae: Synergini). Plant Protection Quarterly, 29 (1), 26-31.

Bozsó M., Tang C.-T., Pénzes Z., Yang M.-M., Bihari P., Pujade-Villar J., Schwéger S. and Melika G. (2015) A new genus of cynipid inquiline, Lithosaphonecrus Tang, Melika \& Bozsó (Hymenoptera: Cynipidae: Synergini), with description of four new species from Taiwan and China. Insect Systematics \& Evolution, 46(1), 79-114. Online published: 45 (2013), 1-36.

Brussino, G., Bosio, G., Baudino, M., Giordano, R., Ramello, F. \& Melika, G. (2002) Nuovo cinipide galligeno in Piemonte. Pericoloso insetto esotico per il castagno europeo. L'Informatore Agrario, 37(Settembre-3 Ottobre), 59-61.

Burks, B.D. (1979) Cynipoidea. Pp. 1045-1107. In: Krombein, K.V., Hurd, Jr., P.D., Smith, D.R. \& Burks, B.D. (eds.). Catalog of Hymenoptera in North America north of Mexico, vol. 1. Smithsonian Institution Press, Washington, D.C.

Camus, A. (1936-1954) Les chenes monographie du genre Quercus (et Lithocarpus) Encyclopédie economique de sylviculture, vols. 6-8. Academie des Sciences, Paris.

Cameron, P. (1902) Description of a new species of gallmaking Cynipidae from the N.W. Himalayas. Entomologist, 1902, 38-39.

Cannon, C.H. \& Manos, P.S. (2003) Phylogeography of Southeast Asian stone oaks (Lithocarpus). Journal of Biogeography, 30, 211-226. 
Cho, D.Y. \& Lee, S.O. (1963) Ecological studies on the chestnut gall wasp, Dryocosmus kuriphilus Yasumatsu, and observation on the damages of the chestnut trees by its insect. Korean Journal of Plant Protection, 2, 47-54 (in Korean).

Cook, J.M., Rokas, A. Pagel, M. \& Stone, G.N. (2002) Evolutionary shifts between host oak species and host plant organs in Andricus gallwasps. Evolution, 56, 1821-1830.

Csóka, G., Stone, G.N. \& Melika, G. (2005) The biology, ecology and evolution of gall wasps. Pp. 573-642. In: A. Raman, C.W. Schaeffer \& T.M. Withers (eds.). Biology, ecology and evolution of gall-inducing arthropods. Science Publishers Inc., Enfield, New Hampshire, USA.

Dalla Torre, K.W. \& Kieffer, J.J. (1910) Cynipidae. Das Tierreich, 24. Berlin, Friedlander \& Sohn, $891 \mathrm{pp}$.

Dettmer, H.S.J. (1934) Vier neue Neuroterus-Arten aus Japan. Broteria, 3, 123-132.

Eady, R.D. \& Quinlan, J. (1963) Hymenoptera: Cynipoidea. Key to families and subfamilies and Cynipinae (including galls). Handbooks for the Identification of British Insects, 8(1a), 1-81.

Edgar, R.C. (2004) MUSCLE: multiple sequence alignment with high accuracy and high throughput. Nucleic Acids Research, 32, 1792-1797.

Fergusson, N.D.M. 1995: The cynipoid families. Pp. 247-265. In: Hanson, P.E. \& Gauld, I.D. (eds.). The Hymenoptera of Costa Rica. Oxford University Press. Oxford, New York, Tokyo.

Folmer, O., Black, M.B., Hoch, W., Lutz, R.A. \& Vrijenhock, R.C. (1994) DNA primers for amplification of mitochondrial cytochrome c oxidase subunit I from metazoan invertebrates. Molecular Marine Biology and Biotechnology, 3, 294-299.

Govaerts, R. \& Frodin, D.G. (1998) World Checklist and Bibliography of Fagales. Royal Botanic Gardens, Kew, 408 pp.

Hancock, J.M., Tautz, D. \& Dover, G.A. (1988) Evolution of the secondary structures and compensatory mutations of the ribosomal RNAs of Drosophila melanogaster. Molecular Biology and Evolution, 5, 393-414.

Harris, R. (1979) A glossary of surface sculpturing. State of California, Department of Food and Agriculture. Occasional Papers of Entomology, 28, 1-31.

Hartig, T. (1843) Zweiter nachtrag zur naturgeschichte der gall-wespen. Zeitschrift für die Entomologie, 4, 395-422.

Hebert, P.D.N., Cywinska, A., Ball, S.L. \& deWaard, J.R. (2003) Biological identifications through DNA barcodes. Proceedings of the Royal Society, 270, 313-321. 
Hubert, F., Grimm, G.W., Jousselin, E., Berry, V., Franc, A. \& Kremer, A. (2014) Multiple nuclear genes stabilize the phylogenetic backbone of the genus Quercus. Systematics and Biodiversity, 1-19.

Ide, T. \& Abe, Y. (2015) A New Species of Dryocosmus Giraud (Hymenoptera: Cynipidae: Cynipini) in Japan and Korea-First Record of Eastern Palearctic Dryocosmus Species Showing Alternation of Generations on Section Cerris Oaks. Proceedings of the Entomological Society of Washington, 117(4), 467-480.

Ide, T. \& Abe, Y. (2016) First Description of Asexual Generation and Taxonomic Revision of the Gall Wasp Genus Latuspina (Hymenoptera: Cynipidae: Cynipini). Annals of the Entomological Society of America, 109, 812-830.

Ide, T., Wachi, N. \& Abe, Y. (2010) Discovery of a New Plagiotrochus Species (Hymenoptera: Cynipidae) Inducing Galls on the Evergreen Oak in Japan. Annals of the Entomological Society of America, 103, 838-843.

Ide, T., Wachi, N. \& Abe, Y. (2012) Three New Species and a New Record of Cycloneuroterus (Hymenoptera: Cynipidae: Cynipini) Inducing Galls on Cyclobalanopsis in Japan. Annals of the Entomological Society of America, 105, 539-549.

Ide, T., Wachi, N. \& Abe, Y. (2013) Description of Two New Species of Dryocosmus (Hymenoptera: Cynipidae: Cynipini) Inducing Galls on the Strictly Asian Subgenus Cyclobalanopsis of the Genus Quercus, With a Key to Species of Dryocosmus in East Asia. Annals of the Entomological Society of America, 106, 18-25.

Kaartinen, R., Stone, G.N., Hearn, J., Lohse, K. \& Roslin, T. (2010) Revealing secret liaisons: DNA barcoding changes our understanding of food webs. Ecological Entomology, 35, 623-638.

Katoh, K., Misawa, K., Kuma, K.-i, \& Miyata, T. (2002) MAFFT: a novel method for rapid multiple sequence alignment based on fast Fourier transform. Nucleic Acids Research, 30, 30593066.

Katoh, K. \& Standley, D.M. (2013) MAFFT Multiple Sequence Alignment Software Version 7: Improvements in Performance and Usability. Molecular Biology and Evolution, 30, 772-780.

Katsuda, T. \& Yukawa, J. (2004) Gall wasps (Hymenoptera: Cynipidae) in Kyushu, Japan. Esakia, 44, 111-123.

Kieffer, J. J. (1906) Description d'un genre nouveau et deux especes nouvelles. Marcellia, 5, $101-110$.

Kos, K., Kriston, É., Melika, G. (2015) Invasive chestnut gall wasp Dryocosmus kuriphilus (Hymenoptera: Cynipidae), its native parasitoid community and association with oak gall wasps in Slovenia. European Journal of Entomology, 112(4), 698-704. 
Kovalev, O.V. (1965) Gall wasps (Hymenoptera, Cynipidae) from the south of the Soviet Far East. Entomologicheskoye Obozrenije, 44(1/2), 46-73. (in Russian) [English translation in Entomological Review, 44, 25-38].

Li, H.M. \& Zhou, Z.K. (2007) Fossil nothofagaceous leaves from the Eocene of western Antarctica and their bearing on the origin, dispersal and systematics of Nothofagus. Science in China, 50 (10), 1525-1535.

Liljeblad, J., Nieves-Aldrey, J.-L., Neser, S. \& Melika, G. (2011) Adding another piece to the cynipoid puzzle: the description of a new tribe, genus and species of gall wasp (Hymenoptera: Cynipidae) endemic to The Republic of South Africa. Zootaxa, 2806, 35-52.

Liljeblad, J. \& Ronquist, F. (1998) A phylogenetic analysis of higher-level gall wasp relationships (Hymenoptera: Cynipidae). Systematic Entomology, 23, 229-252.

Liljeblad, J., Ronquist, F., Nieves-Aldrey, J.-L., Fontal-Cazalla, F., Ros-Farré, P., Gaitros, D. \& Pujade-Villar, J. (2008) A fully web-illustrated morphological phylogenetic study of relationships among oak gall wasps and their closest relatives (Hymenoptera: Cynipidae). Zootaxa, 1796, 1-73.

Linkuo, F. \& Tao, H. (eds.) (1998) Higher plants of China. Volume 4. Qingdao Publishing House, China.

Liu, Z., Yang, X.-H., Zhu, D.-H. \& He, Y.-Y. (2012) A New Species of Saphonecrus (Hymenoptera, Cynipoidea) Associated with Plant Galls on Castanopsis (Fagaceae) in China. Annals of the Entomological Society of America, 105(4), 555-561.

Lu, F.Y., Ou, C.H., Chen, Y.C., Chi, Y.S., Lu, K.C. \& Tseng, Y.H. (2006) Trees of Taiwan. Volume 2. Department of Forestry, National Chung Hsing University, Taichung, Taiwan, 500 pp. (in Chinese).

Mani, M.S. (2000) Plant galls of India. Science Publishers Inc. Enfield, New Hampshire, U.S.A., 477pp.

Manos, P.S., Cannon, C.H. \& Oh, S.-H. (2008) Phylogenetic relationships and taxonomic status of the paleoendemic Fagaceae of western North America: recognition of a new genus, Notholithocarpus. Madrano, 55(3), 181-190.

Manos, P.S., Doyle, J.J. \& Nixon, K.C. (1999) Phylogeny, Biogeography and Processes of Molecular differentiation in Quercus subgenus Quercus (Fagaceae). Molecular Phylogenetics and Evolution, 12, 333-349.

Manos, P.S. \& Stanford, A.M. (2001) The historical biogeography of Fagaceae: tracking the tertiary history of temperate and subtropical forests in the northern hemisphere. International Journal of Plant Science, 162, S77-S93. 
Manos, P.S., Zhou, Z.K. \& Cannon, C.H. (2001) Systematics of Fagaceae: phylogenetic tests of reproductive trait evolution. International Journal of Plant Science, 162 (6), 1361-1379.

Mayr, G. (1872) Die Einmiethler der mitteleuropäischen Eichengallen. Verhandlungen der Zoologisch-Botanischen Geselschaft in Wien, 22, 669-726.

Melika, G. (2006) Gall wasps of Ukraine. Cynipidae. Vestnik Zoologii, Suppl. 21, 1-644.

Melika, G. (2012) Надсем. CYNIPOIDEA - ЦИНИПОИДНЫЕ, или ОРЕХОТВОРКИ. Pp. 139-146. In: Lelej, A. (ed.) Annotated catalogue of the insects of Russian Far East. Volume I. Hymenoptera. Vladivostok, Dalnauka, 635 pp. (in Russian).

Melika, G. \& Abrahamson, W.G. (2002) Review of the World Genera of Oak Cynipid Wasps (Hymenoptera: Cynipidae, Cynipini). Pp. 150-190. In: Melika, G. \& Thuróczy, Cs. (eds.) Parasitic Wasps: Evolution, Systematics, Biodiversity and Biological Control. Agroinform, Budapest.

Melika, G., Ács, Z. \& Bechtold, M. (2004) New species of cynipid inquilines from China (Hymenoptera: Cynipidae: Synergini). Acta Zoologica Academiae Scientiarum Hungaricae, 50, 319-336.

Melika, G., Choi, J.-Y., Pujade-Villar, J., Pénzes, Zs. \& Fülöp, D. (2007) A new species of inquiline cynipid of the genus Ufo Melika \& Pujade-Villar, 2005 from Korea (Hymenoptera: Cynipidae: Synergini). Journal of Asian-Pacific Entomology, 10(3), 197-200.

Melika, G., Pujade-Villar, J., Abe, Y., Tang, C.-T., Nicholls, J., Wachi, N., Ide, T., Yang, M.-M., Pénzes, Zs., Csóka, Gy. \& Stone, G.N. (2010) Palearctic oak gallwasps galling oaks (Quercus) in the section Cerris: re-appraisal of generic limits, with descriptions of new genera and species (Hymenoptera: Cynipidae: Cynipini). Zootaxa, 2470, 1-79.

Melika, G., Pujade-Villar, J., Stone, G.N., Fülöp, D. \& Pénzes, Zs. (2009) New species of cynipid gallwasps of the genus Plagiotrochus Mayr, 1881 from Nepal and Jordan (Hymenoptera: Cynipidae: Cynipini). Acta Zoologica Academiae Scientiarum Hungaricae, 55, 263-264.

Melika, G., Ros-Farré, P., Pénzes, Zs., Ács, Z. \& Pujade-Villar, J. (2005) Ufo abei Melika et Pujade-Villar (Hymenoptera: Cynipidae: Synergini) new genus and new species from Japan. Acta Zoologica Academiae Scientiarum Hungaricae, 51(4), 313-327.

Melika G., Tang C.-T., Nicholls, J.A., Yang, M.-M. \& Stone, G.N. (2011) Four New Species of Dryocosmus gallwasps from Taiwan (Hymenoptera: Cynipidae: Cynipini). ISRN Zoology, Article ID 725180, 1-17.

Melika G., Tang C.-T., Sinclair F., Yang, M.-M., Lohse K., Hearn, J., Nicholls, J.A. \& Stone, G.N. (2013) A new genus of oak gallwasp, Cyclocynips Melika, Tang \& Sinclair (Hymenoptera: Cynipidae: Cynipini), with descriptions of two new species from Taiwan. Zootaxa, 3630, 534548. 
Melika, G., Tang, C.-T., Yang, M.-M., Bihari, P., Bozsó, M. \& Pénzes, Zs. (2012) New species of cynipid inquilines of the genus Ufo Melika \& Pujade-Villar, 2005 (Hymenoptera: Cynipidae: Synergini) Zootaxa, 3478, 143-163.

Monzen, K. (1931) On Japanese gallwasps. Oyo-doubutsugaku-zasshi, 3, 192-200 (in Japanese).

Monzen, K. (1953) Revision of the Japanese gall wasps with the descriptions of new genus, subgenus, species and subspecies (I). Cynipidae (Cynipinae), Hymenoptera. Annual Report of Gakugei Faculty, Iwate University, 5, 15-21.

Monzen, K. (1954) Revision of the Japanese gall wasps with the descriptions of new genus, subgenus, species and subspecies (II). Cynipidae (Cynipinae) Hymenoptera. Annual Report of Gakugei Faculty, Iwate University, 6, 24-38.

Mukaigawa, Y. (1920a) Galls of Aphelomyx [sic] crispulae. Insect World, 24, 21-23 (in Japanese).

Mukaigawa, Y. (1920b) Aphelomyx [sic] glandulifelae. Insect World, 24, 131-133 (in Japanese).

Murakami, Y. (1980) Current topics on the chestnut gall wasp - experience in China. Nogyo oyobi Engei (Agriculture and Horticulture), 55, 249-253 (in Japanese).

Negi, S.S. \& Naithani, H.B. (1995) Oaks of India, Nepal and Bhutan. International Book Distributors, Dhera Dun, India.

Nicholls, J.A., Preuss, S., Hayward, A., Melika, G., Csoka, G., Nieves-Aldrey, J-L., Askew, R.R., Tavakoli, M., Schonrogge, K. \& Stone, G.N. (2010) Concordant phylogeography and cryptic speciation in two Western Palearctic oak gall parasitoid species complexes. Molecular Ecology, 19, 592-609.

Nieves-Aldrey, J.L. (2001) Hymenoptera, Cynipidae. 636 pp. In: Ramos, M.A., Alba, J., Bellés, X., Gosálbez, J., Guerra, A., Macpherson, E., Martín, F., Serrano, J. \& Templado, J. (eds.) Fauna Ibérica. Vol. 16. Museo Nacional de Ciencias Naturales, CSIC, Madrid.

Nieves-Aldrey, J.L. (2005) Notes on the Neotropical species of Synergus (Hymenoptera, Cynipidae), with description of a new species from Colombia. The Canadian Entomologist, 137, 501-508.

Nieves-Aldrey, J.L. \& Butterill, P.T. (2014) First evidence of cynipids from the Oceanian Region: the description of Lithonecrus papuanus a new genus and species of cynipid inquiline from Papua New Guinea (Hymenoptera: Cynipidae, Synergini). Zootaxa, 3846 (2), 221-234.

Nieves-Aldrey, J.L. \& Medianero, E. (2010) Agastoroxenia panamensis, a New Genus and Species of Inquiline Oak Gall Wasps (Hymenoptera: Cynipidae: Synergini) of the Neotropics. Annals of the Entomological Society of America, 103(4), 492-499. 
Nieves-Aldrey, J.L. \& Pujade-Villar, J. (1985) Sobre las especies ibéricas de la Sección 1 (Mayr, 1872) del género Synergus Htg. Eos, 61, 219-237.

Nieves-Aldrey, J.L. \& Pujade-Villar, J. (1986) Sobre las especies ibéricas de la Sección II (Mayr, 1872) del género Synergus Htg. con descripción de una especie nueva (Hym., Cynipidae, Cynipinae). Eos, 62, 137-165.

Nieves-Aldrey, J.L., Liljeblad, J., Hernández Nieves, M., Grez, A. \& Nylander, J.A.A. (2009) Revision and phylogenetics of the genus Paraulax Kieffer (Hymenoptera, Cynipidae) with biological notes and description of a new tribe, a new genus, and five new species. Zootaxa, $2200,1-40$.

Nixon, K.C. \& Crepet, W.L. (1989) Trigonobalanus (Fagaceae): taxonomic status and phylogenetic relationships. American Journal of Botany, 6, 828-841.

Nixon, K.C. (1993) Infrageneric classification of Quercus (Fagaceae) and typification of sectional names. Annals of Science Forest, 50 (Supplement), 25S-34S.

Nordlander, G. (1984) What do we know about parasitic cynipoids (Hymenoptera). Entomologisk Tidskrift, 105, 36-40.

Nylander, J.A.A., Ronquist, F., Huelsenbeck, J.P. \& Nieves Aldrey, J.-L. (2004) Bayesian phylogenetic analysis of combined data. Systematic Biology, 53, 47-67.

Oh, S.-H. \& Manos, P.S. (2008) Molecular phylogenetics and cupule evolution in Fagaceae as inferred from nuclear CRABS CLAW sequences. Taxon, 57, 434-451.

Ohwi, J. (1961) Flora of Japan. Shibundo, Tokyo, 1383 pp. (in Japanese).

Payne, J.A., Green, R.A. \& Lester, C.D. (1976) New nut pest: an oriental chestnut gall wasp in North America. Annual Report of the Northern Nut Growers Association, 67, 83-86.

Payne, J.A., Menke, A.S. \& Schroeder, P.M. (1975) Dryocosmus kuriphilus Yasumatsu, (Hymenoptera: Cynipidae), An Oriental chestnut gall wasp in North America. United States Department of Agriculture. Economic Insect Reports, 25, 903-905.

Pénzes, Zs., Melika, G., Bozsóki, Z., Bihari, P., Mikó, I., Tavakoli, M., Pujade-Villar, J., Fehér, B., Fülöp, D., Szabó, K., Bozsó, M., Sipos, B., Somogy, K. \& Stone, G.N. (2009) Systematic reappraisal of the gall-usurping wasp genus Synophrus Hartig, 1843 (Hymenoptera: Cynipidae: Synergini). Systematic Entomology, 34, 688-711.

Pénzes, Zs., Tang, Ch.-T., Bihari, P., Bozsó, M., Schwéger S. \& Melika, G. (2012) Oak associated inquilines (Hymenoptera, Cynipidae, Synergini). TISCIA monograph series, 11, Szeged (Hungary). 1-76. 
Pujade-Villar, J. (2004) On two valid cynipid species: Saphonecrus gallaepomiformis (Boyer de Fonscolombe, 1832) n. comb. and Synergus facialis Hartig, 1840 (Hym., Cynipidae: Synergini). Buttletí de la Institució Catalana d'História Natural, 72, 110-112.

Pujade-Villar, J., Melika, G., Ros-Farre, P., Ács, Z. \& Csoka, G. (2003) Cynipid inquiline wasps of Hungary, with taxonomic notes on the Western Palearctic fauna (Hymenoptera: Cynipidae, Cynipinae, Synergini). Folia Entomololica Hungarica, 64, 121-170.

Pujade-Villar, J. \& Nieves-Aldrey, J.L. (1990) Revisión de las especies europeas del género Saphonecrus Dalla Torre \& Kieffer, 1910 (Hymenoptera, Cynipoidea, Cynipidae). Butlletí de la Institució Catalana d'História Natural Secció Zoologia, 8, 45-55.

Pujade-Villar, J. \& Nieves-Aldrey, J.L. (1993) Revisión de las especies europeas del género Ceroptres Htg. 1840 (Hymenoptera: Cynipidae). Boletin de la Asociación Espanola de Entomologia, 17, 49-63.

Pujade-Villar, J., Ros-Farré, P. \& Abe, Y. (2002) Biological and morphological differences of two closely related species of Synergus Hartig from Japan (Hymenoptera: Cynipidae). Pp. 278284. In: Melika, G. \& Thuroczy, Cs. (eds.) Parasitic Wasps: Evolution, Systematics, Biodiversity and Biological Control. Agroinform, Budapest.

Pujade-Villar, J. \& Wang, J. (2012) A new species of the genus Trichagalma Mayr from China (Hym.: Cynipidae). Orsis, 26, 91-101.

Pujade-Villar, J., Wang, Y.-P., Chen, X.-X. \& He, J.H. (2014b) Taxonomic review of East Palearctic species of Synergus section I, with description of a new species from China (Hymenoptera: Cynipidae: Cynipinae). Zoological Systematics, 39(4), 534-544.

Pujade-Villar, J., Wang, Y. \& Guo, R. (2014c) Description of a new species of Saphonecrus Dalla Torre \& Kieffer from China (Hymenoptera: Cynipidae: Synergini). Entomologica Fennica, $25,43-48$.

Pujade-Villar, J., Wang, Y., Guo, R. \& Chen, X.-X. (2014a) New species of gallwasps inducing in Quercus fabri and its inquiline (Hymenoptera: Cynipidae) in China. Zoological Systematics, 39(3), 417-423.

Pujade-Villar, J., Wang, Y., Liu, Z. \& Guo, R. (2015) Descriptions of two new species of Neuroterus Hartig from China (Hymenoptera: Cynipidae). Entomologica Fennica, 27, 23-32.

Pujade-Villar, J., Wang, Y., Tang, G., Shen, J. \& Ferrer-Suay, M. (2016) Andricus mukaigawae and A. kashiwaphilus from China with remarks of morphological differences and inquilines (Hymenoptera, Cynipidae). Butlleti de la Institució Catalana d'Historia Natural, 80, 17-24. 
Radócz, L., Szilágyi, A., Nagy, M., Kovács, G. \& Melika, G. (2015) Asian sweet chestnut gallwasp, Dryocosmus kuriphilus (Hymenoptera, Cynipidae): first record for Romania. NorthWestern Journal of Zoology (online): art.157201.

Ritchie, A.J. (1984) A review of the higher classification of the inquiline gall wasps (Hymenoptera: Cynipidae) and a revision of the Nearctic species of Periclistus Förster. Ph.D. Thesis. Carleton University, Ottawa, 365 pp.

Ronquist, F. (1994) Evolution of parasitism among closely related species: phylogenetic relationships and the origin of inquilinism in gall wasps (Hymenoptera, Cynipidae). Evolution, 48(2), 241-266.

Ronquist, F. (1999) Phylogeny, classification and evolution of the Cynipoidea. Zoologica Scripta, 28(1-2), 139-164.

Ronquist, F. \& Huelsenbeck, J.P. (2003) MrBayes 3: Bayesian phylogenetic inference under mixed models. Bioinformatics, 19(12), 1572-1574.

Ronquist, F. \& Liljeblad, J. (2001) Evolution of the gall wasp-host-plant association. Evolution, $55,2503-2522$.

Ronquist, F. \& Nordlander, G. (1989) Skeletal morphology of an archaic cynipoid, Ibalia rufipes (Hymenoptera: Ibaliidae). Entomological Scandinavica, supplement 33, 1-60.

Ronquist, F., Nieves-Aldrey, J.-L., Buffington, M.L., Liu, Zh., Liljeblad, J. \& Nylander, J.A.A. (2015) Phylogeny, Evolution and Classification of Gall Wasps: The Plot Thickens. PLOS One, DOI:10.1371/journal.pone.0123301

Ronquist, F., Teslenko, M., van der Mark, P., Ayres, D.L., Darling, A., Höhna, S., Larget, B., Liu, L., Suchard, M.A. \& Huelsenbeck, J.P. (2012) MRBAYES 3.2: Efficient Bayesian phylogenetic inference and model selection across a large model space. Systematic Biology, 61(3), 539-542.

Sadeghi, S.E., Melika, G., Pujade-Villar, J., Pénzes, Zs., Ács, Z., Bechtold, M., Assareh, M.H., Tavakoli, M., Yarmand, H., Askary, H., Stone, G.N., Azizkhani, E., Zargaran, M.R., Aligolizade, D., Barimani, H. \& Dordaei, A.A. (2006) Oak cynipid gall inquilines of Iran (Hym.: Cynipidae: Synergini), with description of new species. Journal of the Entomological Society of Iran, 25(2), 15-50.

Sakagami, S. (1949) Notes on Saphonecrus connatus (Hartig) (Hym., Cynipidae). Matsumushi, 3, 112-115 (in Japanese).

Schwéger, S., Melika, G., Tang, C.-T., Bihari, P., Bozsó, M., Stone, G.N., Nicholls, J.A. \& Pénzes, Z. (2015a) New species of cynipid inquilines of the genus Synergus (Hymenoptera: Cynipidae: Synergini) from the Eastern Palearctic. Zootaxa, 3999 (4), 451-497. 
Schwéger, S., Melika, G., Tang, C.-T., Yang, M.-M., Stone, G.N., Nicholls, J.A., Sinclair, F., Hearn, J., Bozsó, M. \& Pénzes Z. (2015b) New species of cynipid inquilines of the genus Saphonecrus (Hymenoptera: Cynipidae: Synergini) from the Eastern Palearctic, with a reappraisal of known species world-wide. Zootaxa, 4054(1), 1-84.

Shinji, O. (1938) On a new species of Cynipidae infesting terminal branches of Quercus spp. in Japan. Kontyu, 12, 203-204 (in Japanese).

Shinji, O. (1940) On two new species of Cinipidae [sic] (Hymenoptera). Insect World, 44, 258260 (in Japanese).

Shinji, O. (1941) Another new species of Cynipid from Japan. Insect World, 45, 34-35 (in Japanese).

Stone, G.N., Hernandez-Lopez, A., Nicholls, J.A., di Pierro, E., Pujade-Villar, J., Melika, G. \& Cook, J.M. (2009) Extreme host plant conservatism during at least 20 million years of host plant pursuit by oak gallwasps. Evolution, 63, 854-869.

Stone, G. N., Schönrogge, K. Atkinson, R. J. Bellido, D. \& Pujade-Villar, J. (2002) The population biology of oak gall wasps (Hymenoptera : Cynipidae). Annual Review of Entomology, 47, 633-668.

Tamura, M. (1962) Occurrence of the chestnut gall wasp in Korea. Kontyu, 30, 251 (in Japanese).

Tang, C.-T. (2015) Host-plant diversity and systematics of oak gallwasps (Hymenoptera: Cynipidae) in Taiwan. Ph.D. Thesis. Department of Entomology, National Chung Hsing University. $202 \mathrm{pp}$.

Tang, C.-T., Melika, G., Nicholls, J.A., Yang, M.-M. \& N, S.G. (2011a) A new genus of oak gallwasps, Cycloneuroterus Melika \& Tang, with the description of five new species from Taiwan (Hymenoptera: Cynipidae: Cynipini). Zootaxa, 3008, 33-62.

Tang, C.-T., Melika, G., Yang, M-M., Nicholls, J. \& Stone, G.N. (2011b) New species of oak gallwasps from Taiwan (Hymenoptera: Cynipidae: Cynipini). Zootaxa, 2865, 37-52.

Tang, C.-T., Melika, G.,Yang, M.-M., Nicholls, J., Csóka, Gy. \& Stone, G. N. (2009) First record of an Andricus oak gallwasp from the Oriental Region: a new species from Taiwan (Hymenoptera: Cynipidae: Cynipini). Zootaxa, 2175, 57-65.

Tang, C.-T., Mikó, I., Nicholls, J.A., Schwéger, Sz., Yang, M.-M., Stone, G.N., Sinclair, F., Bozsó, M., Melika, G. \& Pénzes, Zs. (2016c, accepted) New Dryocosmus Giraud species associated with Cyclobalanopsis and non-Quercus host plants from the Eastern Palearctic (Hymenoptera: Cynipidae: Cynipini). Journal of Hymenoptera Research 
Tang, C.-T., Sinclair, F., Hearn, J., Yang M.-M., Stone, G.N., Nicholls J.A., Schwéger, S. \& Melika, G. (2016a) Eight new species of Cycloneuroterus Melika \& Tang gallwasps from Taiwan and mainland China (Hymenoptera: Cynipidae: Cynipini). Zootaxa, 4088 (4), 451-488.

Tang, C.-T., Sinclair, F. \& Melika, G. (2012a) A new Latuspina Monzen oak gallwasp species from Taiwan (Hymenoptera: Cynipidae: Cynipini). Journal of Asia-Pacific Entomology, 15, 573-577.

Tang, C.-T., Sinclair, F., Yang, M.-M. \& Melika, G. (2012b) A new Andricus Hartig oak gallwasp species from China (Hymenoptera: Cynipidae: Cynipini). Journal of Asia-Pacific Entomology, 15, 601-605.

Tang, C.-T., Yang M.-M., Stone, G.N., Nicholls J.A., Melika, G. (2016b) A new Plagiotrochus Mayr oak gallwasp species from Taiwan (Hymenoptera: Cynipidae: Cynipini). Journal of AsiaPacific Entomology, 19, 531-536.

Ueno, W. (2006) Occurrence and control of chestnut gall wasp in Nepal. Shokubutsu Boeki (Plant Protection), 60, 510-512 (in Japanese).

van Noort, S., Stone, G.N., Whitehead, V.B. and Nieves-Aldrey, J.L. (2007) Biology of Rhoophilus loewi (Hymenoptera: Cynipoidea: Cynipidae), with implications for the evolution of inquilinism in gall wasps. Biological Journal of the Linnaean Society, 90, 153-172.

Wachi, N. \& Abe, Y. (2009) Taxonomic status of the oak gall wasp Callirhytis hakonensis Ashmead (Hymenoptera: Cynipidae), a candidate for native host of Torymus beneficus Yasumatsu et Kamijo (Hymenoptera: Torymidae), with a description of its sexual generation. P. 33. In: Moriya, S. (Ed.), A Global Serious Pest of Chestnut Trees: Yesterday, Today and Tomorrow. National Agricultural Research Center, Tsukuba, Ibaraki, Japan.

Wachi, N. \& Abe, Y. (2010) Taxonomic status of the oak gall wasp Callirhytis hakonensis Ashmead (Hymenoptera: Cynipidae), with description of the sexual generation. Annals of the Entomological Society of America, 103, 322-326.

Wachi, N., Abe, Y., Inomata, N., Szmidt, A.E. \& Tachida, H. (2012) Speciation history of three closely related oak gall wasps, Andricus mukaigawae, A. kashiwaphilus, and A. pseudoflos (Hymenoptera: Cynipidae) inferred from nuclear and mitochondrial DNA sequences. Molecular Ecology, 21, 4681-4694.

Wachi, N., Ide, T. \& Abe, Y. (2011a) Taxonomic status of two species of Andricus (Hymenoptera: Cynipidae) described by Shinji (1940, 1941) as gall inducers on Cyclobalanopsis. Annals of the Entomological Society of America, 104 (4), 620-626.

Wachi, N., Ide, T. \& Abe, Y. (2011b) A new inquiline species of Saphonecrus (Hymenoptera: Cynipidae: Synergini) associated with cecidomyiid galls on oak trees in Japan. Annals of the Entomological Society of America, 104 (3), 369-373. 
Wang, Y.-P., Chen, X.-X., Pujade-Villar, J., Wu, H. \& He, J.-H. (2010) The genus Saphonecrus Dalla Torre et Kieffer, 1910 (Hymenoptera: Cynipidae) in China, with descriptions of two new species. Biologia (Section Zoology), 65 (6), 1034-1039.

Wang, J., Cui, J., Wu, San-An \& Pujade-Villar, J. (2016) Description of the sexual generation of Trichagalma acutissima (Hymenoptera: Cynipidae) and notes on its heterogonic life cycle. Journal of Asia-Pacific Entomology, 19, 405-413.

Wang, Y.-P., Guo, R. \& Chen, X.-X. (2013) A new species of Andricus oak gallwasp from China (Hymenoptera: Cynipidae: Cynipini). Biologia (Section Zoology), 68(5), 974-978.

Wang, Y., Guo, R., Pujade-Villar, J., Wang, S. \& Chen, X.-X. (2016) Review of the genus Latuspina (Hymenoptera: Cynipidae), with descriptions of two new species and their host galls. Zoological Systematics, 41(1), 82-88. DOI: 10.11865/zs.201605.

Weih C. (1965) Preliminary studies on Cynips mukaigawae. Kunchong-zhishi, 9, 160-162 (in Chinese).

Weld, H.H. (1926) Field Notes on Gall-inhabiting Cynipid Wasps with descriptions of new species. Proceedings of the U.S. National Museum, 68 (10), 1-131.

Weld, H.H. (1944) New American Cynipids from Galls. Proceedings of the U.S. National Museum, 95, 1-24.

Weld, L.H. (1952) Cynipoidea (Hym.) 1905-1950. Ann Arbor, Michigan. (Privately printed). $351 \mathrm{pp}$.

Wiebes-Rijks, A.A. (1979) A character analysis of the species of Synergus Hartig, Section II (Mayr, 1872) (Hymenoptera, Cynipidae). Zoologische Mededelingen, 53 (28), 297-321.

Yang, X.-H., Zhu, D.-H., Liu, Zh., Zhao, L. (2012) Sequencing and phylogenetic analysis of the wasp gene of Wolbachia in three geographic populations of an oak gallwasp, Andricus mairei (Hymenoptera: Cynipidae), from Hunan, South China. Acta Entomologica Sinica, 55 (2), 247254.

Yasumatsu, K. (1951) A new Dryocosmus injurious to chestnut trees in Japan (Hymenoptera, Cynipidae). Mushi, 22, 89-92.

Yukawa, J. \& Masuda, H. (eds.) (1996) Insect and mite galls of Japan in colors. ZenkokuNouson-Kyoukai, Tokyo, 826 pp. (in Japanese).

Zhou, Z.-K. (1992) Origin, phylogeny, and dispersal of Quercus from China. Acta Botanica Yunnanica, 14, 227-236.

Zhou, Z.-K. (1993) The fossil history of Quercus. Acta Botanica Yunnanica, 15, 21-33.

Zhu, D.-H., Liu, Z., Lu, P.-F., Yang, X.-H., Su, C.-Y. \& Liu, P. (2015) New Gall Wasp Species Attacking Chestnut Trees: Dryocosmus zhuili n. sp. (Hymenoptera: Cynipidae) on Castanea henryi from Southeastern China. Journal of Insect Science, 15(1), 156, 1-7; DOI: 10.1093/jisesa/iev118. 


\section{SUMMARY}

Cynipidae lies within the superfamily Cynipoidea of the Hymenoptera, which includes approximately 3000 described species (Fergusson 1995, Ronquist 1999). Cynipids are divided into two main trophic groups: the gall inducers, and the gall-associated inquilines. Recently, the higher-level taxonomy of Cynipidae has been changed, the family was divided into 12 monophyletic tribes from which 4 include inquilines (Ronquist et al. 2015).

The first part of our research focused on the inquiline cynipids, tribe Synergini sensu stricto, which attack hosts in the gallwasp tribe Cynipini (oak gallwasps). This tribe consits of seven genera, Agastoroxenia, Saphonecrus, Synergus, Synophrus, Ufo, Lithosaphonecrus and Rhoophilus (Bozsó et al. 2014, Ronquist et al. 2015, Schwéger et al. 2015a, b) of which Synergus and Saphonecrus were investigated in details. Our aim was to start to establish the phylogenetic relationships within the Synergini sensu stricto of which the necessary first step was to establish their morphotaxonomy, which included the description of new species of Synergus and Saphonecrus genera with the re-appraisal of all known EP Synergus and worldwide Saphonecrus species. New key to all EP Synergus species and to world known Saphonecrus species with the description of new species was given.

The phylogenetic analysis supported the monophyly of the large genus Synergus while the monophyly of Saphonecrus was rejected. However, recent studies revealed contradictions between the morphological discrimination and molecular phylogeny on species level, especially within both genera (Pénzes et al. 2009, Ács et al. 2010, Ronquist et al. 2015, Stone et al. 2002). All the newly described EP Saphonecrus and Synergus species are supported by distinct mitochondrial DNA haplotypes. On the other hand, some contradiction between morphologybased taxonomy and molecular phylogeny still remained considering the classification (Schwéger et al. 2015a, b).

Fifteen new species of Saphonecrus were described, Saphonecrus chinensis Tang \& Schwéger, S. gilvus Melika \& Schwéger, S. globosus Schwéger \& Tang, S. leleyi Melika \& Schwéger, S. lithocarpii Schwéger \& Melika, S. longinuxi Schwéger \& Melika, S. morii Schwéger \& Tang, S. nantoui Tang, Schwéger \& Melika, S. nichollsi Schwéger \& Melika, S. pachylomai Schwéger, Tang \& Melika, S. robustus Schwéger \& Melika, S. saliciniai Melika, Tang \& Schwéger, S. shanzhukui Melika \& Tang, S. symbioticus Melika \& Schwéger, and S. taitungi Schwéger, Tang \& Melika, from the Eastern Palearctic. The status of earlier described 
species was re-evaluated. The current number of valid Saphonecrus species worldwide is raised to 36. Data on the diagnoses, biology, distribution, host galls and host plants of each species are given. The status of some earlier described Saphonecrus species was also discussed (Schwéger et al. 2015a). Morphology based delimitation completed our earlier molecular phylogenetic results (Bozsó et al. 2014, 2015) (Schwéger et al. 2015a, b). Host plant associations for the most of 36 Saphonecrus species are given also. 12 species develop on Quercus subgenus Cyclobalanopsis, 4 - 4 species on Quercus section Quercus and Lithocarpus, 5 species on Quercus section Cerris. In the Nearctic Region 2 species are known which associate with Quercus section Lobatae.

Eight new species of Synergus were described, Synergus symbioticus Schwéger \& Melika, S. formosanus Schwéger \& Melika, S. khazani Melika \& Schwéger, S. abei Melika \& Schwéger, S. belizinellus Schwéger \& Melika, S. ishikarii Melika \& Schwéger, S. changtitangi Melika \& Schwéger and S. kawakamii Tang \& Melika, from the Eastern Palearctic. Descriptions, diagnoses, biology, and host associations for the new species and a key to all known Eastern Palearctic Synergus species are given. The discrimination of all taxa is supported by morphological and molecular data. We discussed the status of all previously described Eastern Palearctic Synergus species, and provided validation and synonymization of some species. Three Saphonecrus species were transferred to Synergus: Synergus brevis (Weld), Synergus hupingshanensis (Liu, Yang \& Zhu), and Synergus yukawai (Wachi, Ide \& Abe). The current phylogenetic and morphological examination of the Eastern Palearctic Synergus supported Ács et al. (2010)'s conclusion that palearctic Synergus comprise a monophyletic group, with Eastern Palearctic species nested among the Western Palearctic species. There is thus no evidence that Eastern and Western Palearctic Synergus species represent independent regional radiations. Of 14 Eastern Palearctic Synergus species for which the host gall and host plant associations are known, 11 species associate with hosts developing on Quercus subgenus Quercus. One species, S. itoensis is a gall-inducer on acorns of Quercus subgenus Cyclobalanopsis. Two Synergus species with opened or partially opened radial cell of the fore wing, S. kawakamii and $S$. castaneus, could not be placed reliably on the phylogenetic tree.

The second part of our work is focused on the gathering, pulling together all the fragmented data on the biology, ecology, phenology, life-cycles and taxonomic history of all Eastern Palearctic (EP) Cynipini (oak gallwasp) species (unpublished results). There are only few Cynipidae (Hymenoptera) reviews on the EP species (e.g. Dalla Torre \& Kieffer 1910, Abe 
et al. 2007). Both are out of time, since then dozens of new species were described and a large number of nomenclatorial changes have been done. Nowadays, there is no faunistic review on EP Cynipini, all the data is fragmented, thus a compilation of a complete Cynipini (Cynipidae, Cynipoidea, Hymenoptera) annotated list for the EP (including few species known from the Oriental Region) is useful. The most completed annotated list for EP Cynipini, with a list of species with uncertain status is given. 


\section{8. ÖSSZEFOGLALÓ}

A Cynipoidea családsorozaton belül, a gubacsdarazsak (Cynipidae) családjába mintegy 3000 leírt faj tartozik (Fergusson 1995, Ronquist 1999). A gubacsdarazsak két fö trofikai csoportra bonthatók: gubacsképzők és inkvilin gubacsdarazsak. A Cynipidae családon belül ma 12 monofiletikus tribust különítünk el, melyek közül 4 tribusban találhatóak inkvilinek (Ronquist és mtsai 2015).

Kutatásunk első nagy témaköre az inkvilin gubacsdarazsak, a Synergini sensu stricto tribus, mely fajok gazdái elsősorban a Cynipini tribusba sorolt tölgy gubacsdarazsak. Ezen belül $7 \mathrm{nem}$ (genus) különíthetö el, az Agastoroxenia, Saphonecrus, Synergus, Synophrus, Ufo, Lithosaphonecrus és Rhoophilus (Ronquist és mtsai 2015, Schwéger és mtsai 2015a, b), melyek közül a Saphonecrus és Synergus nemet vizsgáltuk részletesen. Munkánk során célunk volt a Synergini sensu stricto tribuson belüli filogenetikai kapcsolatok feltérképezése, melynek első fontos lépése a fajcsoportok morfotaxonómiai alapú elkülönítése volt, beleértve a Synergus és Saphonecrus nemen belüli új fajok leírását, valamint helyzetük újraértékelését, kiegészítve az eddig ismert összes Saphonecrus és összes kelet-palearktikus Synergus fajjal. Továbbá fontosnak tartottunk egy új, komplex és fajgazdag határozó kulcs megalkotását az eddig leírt keletpalearktikus Synergus és összes Saphonecrus faj esetében az új fajok részletes leírásával kiegészítve.

A filogenetikai elemzés támogatja a fajgazdag Synergus nem monofiletikusságát, a Saphonecrus viszont nem monofiletikus (Pénzes és mtsai 2009, Ács és mtsai 2010, Ronquist és mtsai 2015, Stone és mtsai 2002). Azonban az újabb tanulmányok szerint ellentmondások vannak a faji szintủ morfológiai alapú elkülönítés és a filogenetikai elemzés eredményének összevetése között, különös tekintettel az általunk vizsgált két nemre (Pénzes és mtsai 2009, Ács és mtsai 2010, Ronquist és mtsai 2015). Az összes leírt új Kelet-Palearktikus Saphonecrus és Synergus faj jól elkülönül a mitokondriális DNS haplotípusok alapján. Másrészről több a morfológiai alapú taxonómia és a molekuláris filogenetikai elemzés eredményei közötti bizonytalanság változatlanul fennáll (Schwéger és mtsai 2015a, b).

A Saphonecrus nem esetében tizenöt új fajt írtunk le a Kelet-Palearktikumban, melyek a következök: Saphonecrus chinensis Tang \& Schwéger, S. gilvus Melika \& Schwéger, S. globosus Schwéger \& Tang, S. leleyi Melika \& Schwéger, S. lithocarpii Schwéger \& Melika, S. 
longinuxi Schwéger \& Melika, S. morii Schwéger \& Tang, S. nantoui Tang, Schwéger \& Melika, S. nichollsi Schwéger \& Melika, S. pachylomai Schwéger, Tang \& Melika, S. robustus Schwéger \& Melika, S. saliciniai Melika, Tang \& Schwéger, S. shanzhukui Melika \& Tang, S. symbioticus Melika \& Schwéger, éd S. taitungi Schwéger, Tang \& Melika. A korábban leirt fajok értékelését, besorolását is elvégeztük. Jelenleg a Saphonecrus nemen belül 36 fajt ismerünk, részletesen tárgyaltam a fajok diagnózisát, elterjedését, gubacs- és növénygazda kapcsolatait, valamint a korábban leírt Saphonecrus fajokkal való összehasonlítását (Schwéger és mtsai 2015a). Korábbi molekuláris filogenetikai eredményeinket részletes morfológia alapú elkülönítéssel is kiegészítettük (Bozsó és mtsai 2014, 2015, Schwéger és mtsai 2015a, b). A legtöbb leírt Saphonecrus faj növénygazdája ismert. Közülük 12 faj a Quercus alnem Cyclobalanopsis fajain, 4 - 4 faj a Quercus alnem Quercus szekció fajain és Lithocarpus fajokon, 5 faj pedig a Quercus alnem Cerris szekció fajain található meg. A nearktikus régióban csak 2 fajnak ismert a növénygazdája, melyek a Quercus alnem Lobatae szekcióba tartozó tölgyek.

A Synergus nem esetében 8 új fajt írtunk le a Kelet-Palearktikumban, melyek a következők: Synergus symbioticus Schwéger \& Melika, S. formosanus Schwéger \& Melika, S. khazani Melika \& Schwéger, S. abei Melika \& Schwéger, S. belizinellus Schwéger \& Melika, S. ishikarii Melika \& Schwéger, S. changtitangi Melika \& Schwéger és S. kawakamii Tang \& Melika. Dolgozatomban tárgyaltam az új fajok leírását, diagnózisát, biológiáját, gubacs- és növénygazda kapcsolatait, valamint megalkottuk a Kelet-Palearktikus Synergus nem összes faját magába foglaló határozó kulcsát. Valamennyi tárgyalt taxon elkülönítését a molekuláris és morfológiai adataink egyaránt alátámasztották. vizsgáltuk a korábban leírt Kelet- Palearktikus Synergus fajok jelenlegi helyzetét, szinonimizációját az általunk leírt új fajokkal összevetve. A Saphonecrus nemből három faj került át a Synergus nembe, ezek a következők: Synergus brevis (Weld), Synergus hupingshanensis (Liu, Yang \& Zhu), és Synergus yukawai (Wachi, Ide \& Abe). A Kelet-Palearktikus Synergus klád esetében a molekuláris és morfológiai vizsgálataink alátámasztják a korábban Ács és mtsai (2010) által kapott eredményeket, mely szerint a kelet és nyugat palearktikus Synergus együtt egy monofiletikus csoportot alkot. Nincs bizonyíték a Kelet- és Nyugat-Palearktikus Synergus fajok független regionális eredetére. A 14 eddig leírt Kelet-Palearktikus Synergus faj közül, melynek ismert a gubacs- és növénygazdája is, 11 faj a Quercus alnem Quercus tölgy szekció fajain találhatóak meg. Egy faj, a S. itoensis, a Quercus (Cyclobalanopsis) alnembe tartozó tölgyek makkjaiban képez gubacsot. A Synergus két tagját, $a$ 
S. kawakamii és a S. castaneus fajokat, melyek elülső szárnyán lévő radiális sejt részben vagy teljesen nyitott, nem tudtuk egyértelmüen elhelyezni a génfán.

A munkánk második fő témája a Kelet-Palearktikus Cynipini (tölgy gubacsdarázs) tribusba tartozó fajok biológiájának, ökológiájának, fenológiájának, életciklusainak, és taxonómiai történetüknek összegzése (leközlés alatt álló eredmények), mely adatok számos kis közleményben voltak fellelhetőek. Jelenleg néhány összefoglaló dolgozat áll rendelkezésünkre a Kelet-Palearktikumban elterjedt Cynipidae fajokról (pl. Dalla Torre \& Kieffer 1910, Abe és mtsai 2007). Ezek közül a müvek közül a legtöbb elavult az azóta leírt új fajok és a nevezéktan gyakori és gyors változása miatt. Így hasznosnak bizonyult egy összegző lista létrehozása a Kelet-Palearktikus Cynipini (Cynipoidea, Hymenoptera) tribus jelenleg leírt fajairól (belevéve a néhány Orientális Régióból ismert fajt is). A Kelet-Palearktikus Cynipini tribus részletes listája kiegészítve a bizonytalan státuszú fajokkal a dolgozat függelékének 9.2. és 9.3. részében található (leközlés alatt álló eredmények). 
9. APPENDICES 
9.1. Morphological peculiarities and hosts of Lithosaphonecrus, Rhoophilus, Synophrus, Ufo, Saphonecrus and Synergus

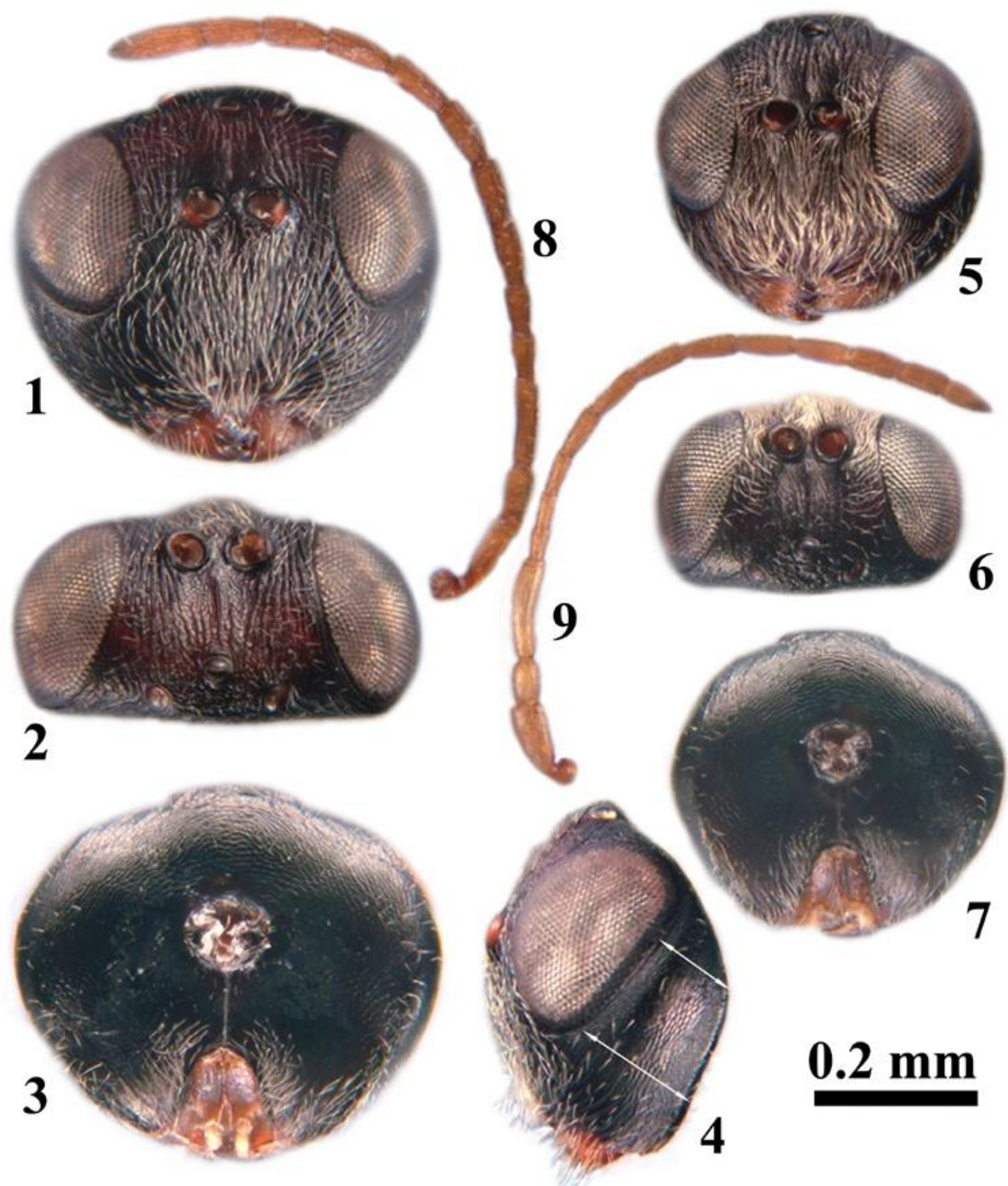

FIGURES A1-A9. Lithosaphonecrus dakengi: 1-4, head, female: 1, frontal view, 2, dorsal view, 3, posterior view, 4, lateral view. 5-7, head, male: 5, frontal view, 6, dorsal view, 7, posterior view. 8-9, antenna: 8, female, 9 , male. 


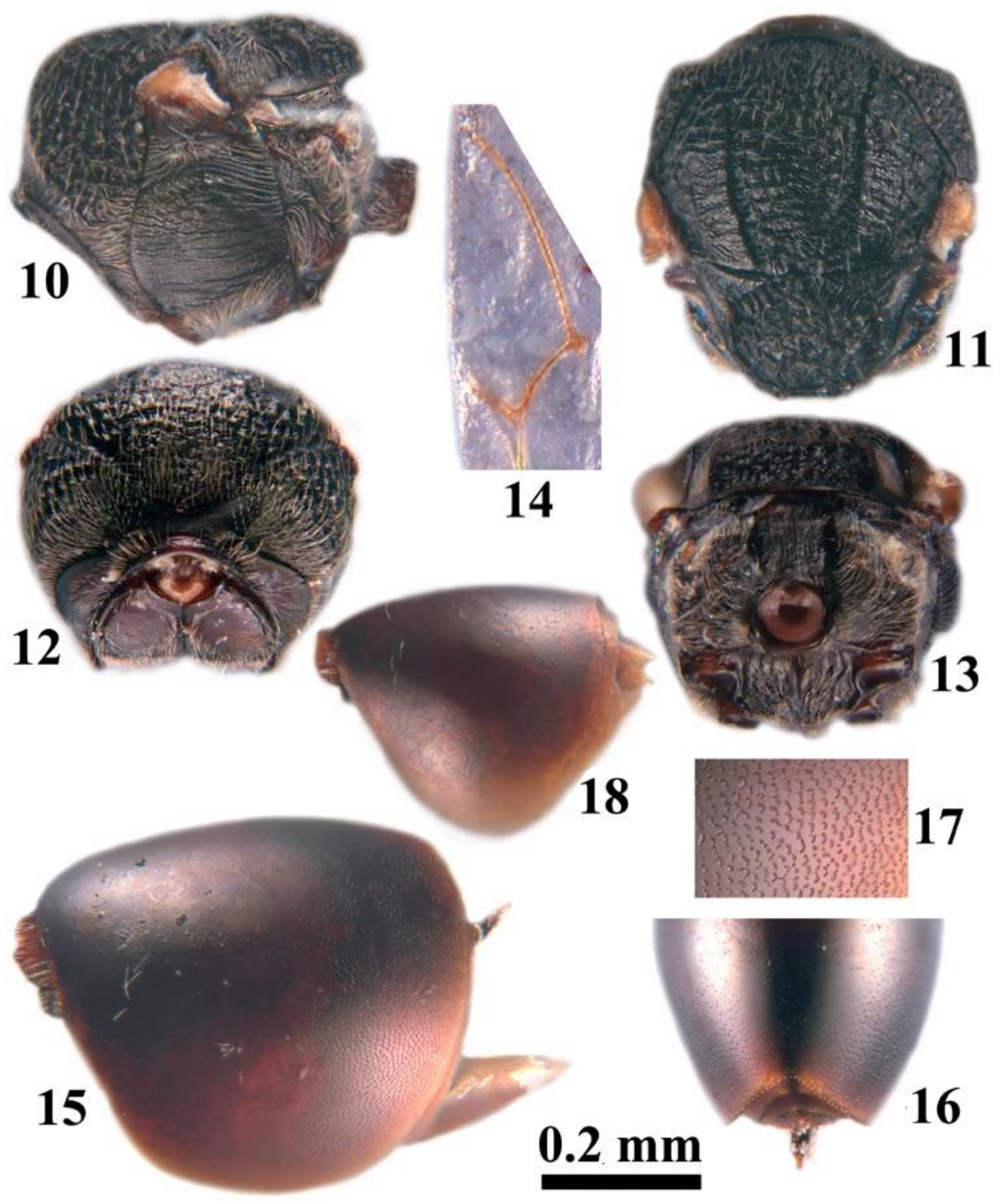

FIGURES A10-A18. Lithosaphonecrus dakengi: 10-17, female: 10, mesosoma, lateral view, 11, mesosoma, dorsal view, 12, mesosoma, frontal view, 13, metascutellum and propodeum, posterodorsal view, 14, fore wing, part, 15, metasoma, lateral view, 16, metasoma, posterior part, dorsal view, 17, surface sculpture of posterior end of metasomal tergite $2+3$, part. 18, metasoma, male, lateral view. 

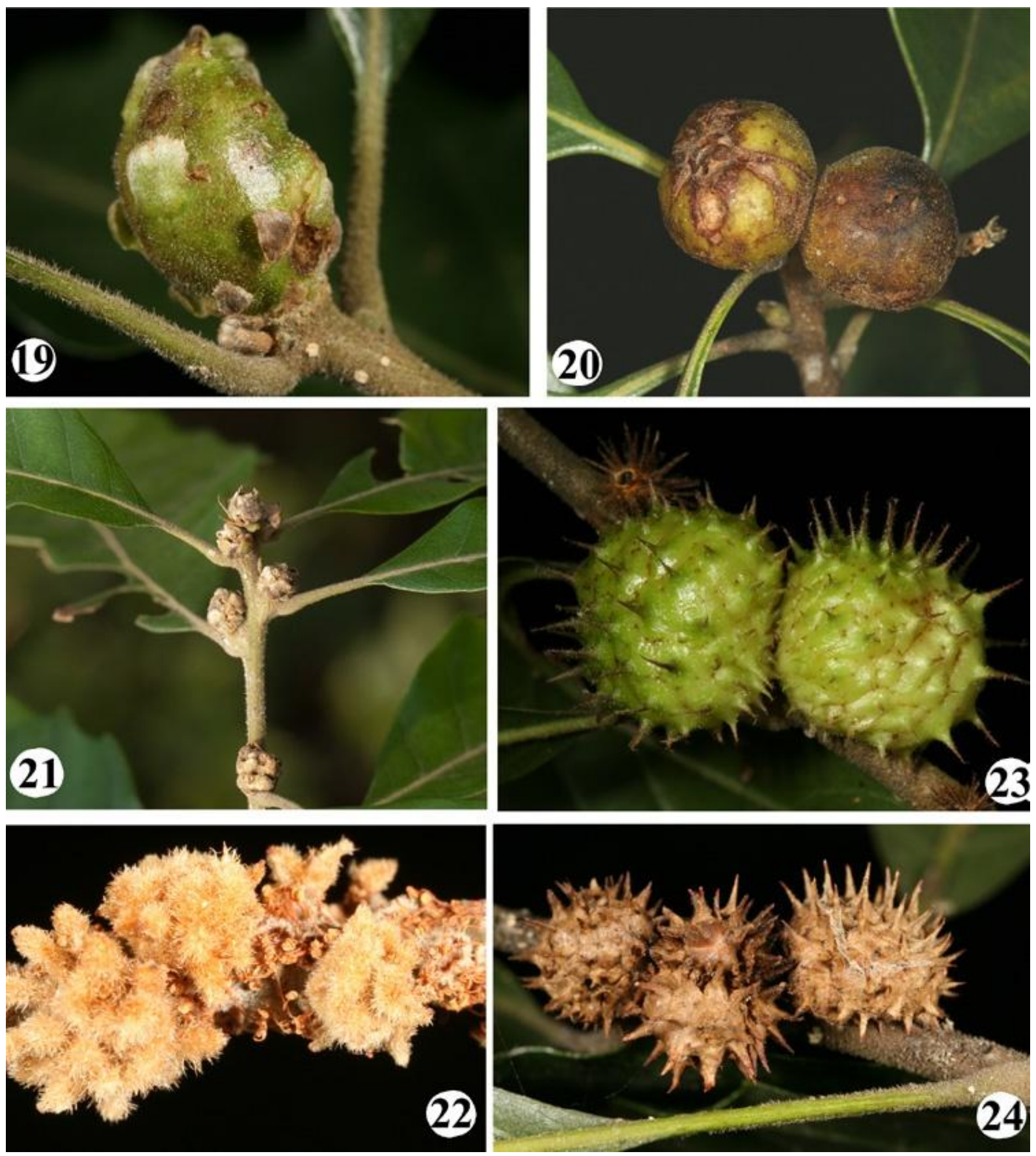

FIGURES A19-A24. Host galls of Lithosaphonecrus spp.: 19, undescribed bud gall, 20, undescribed bud gall, 21, undescribed bud gall, 22, undescribed catkin gall, 23-24, a sea-urchinlike detachable stem gall on twigs: 23, young growing galls, 24, mature galls (photos by G. Csóka and C.-T. Tang). 


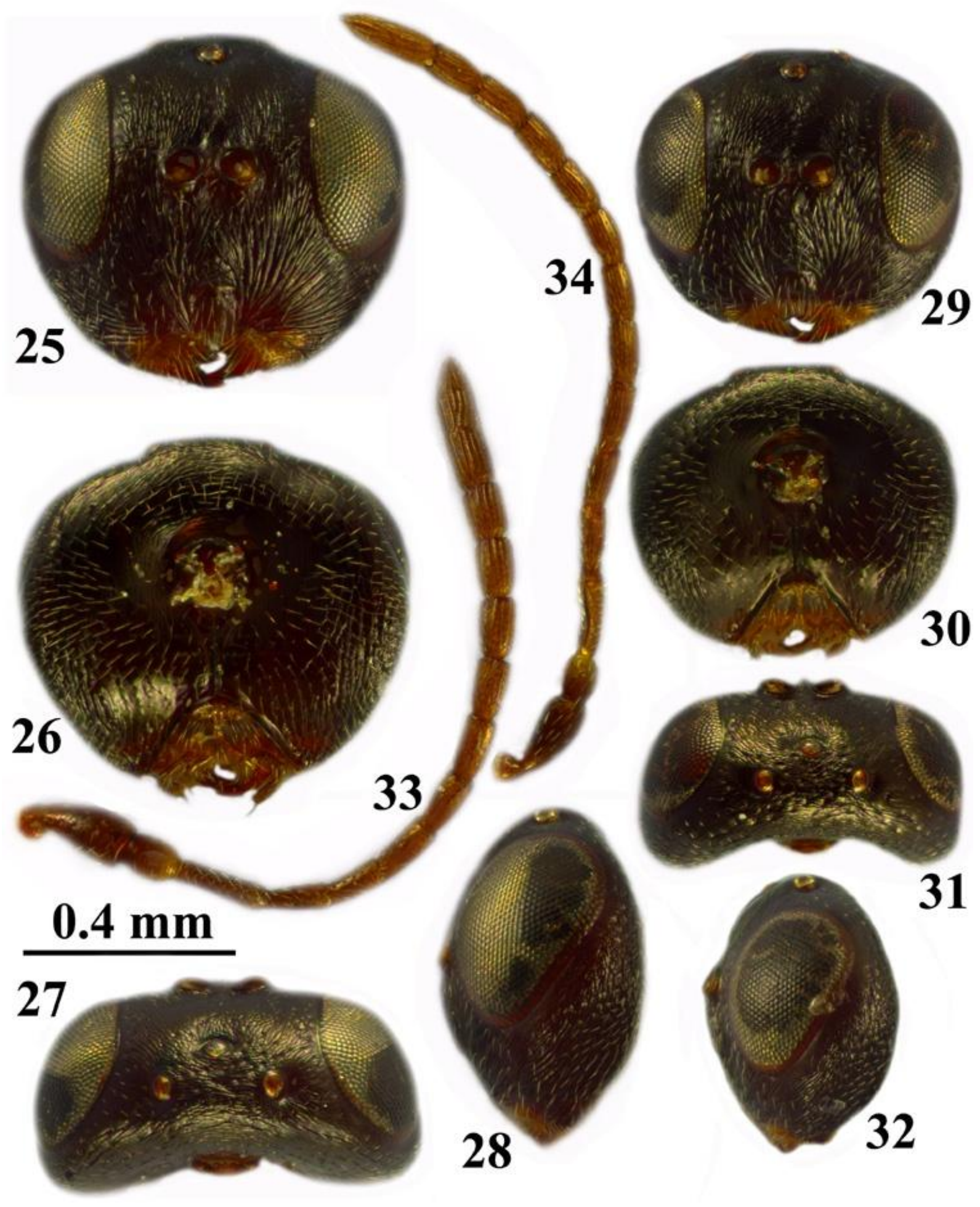

FIGURES A25-A34. Rhoophilus loewi: 25-28, head, female: 25, frontal view, 26, posterior view, 27, dorsal view, 28, lateral view. 29-32, head, male: 29, frontal view, 30, posterior view, 31, dorsal view, 32, lateral view. 33-34, antenna: 33, female, 34, male. 


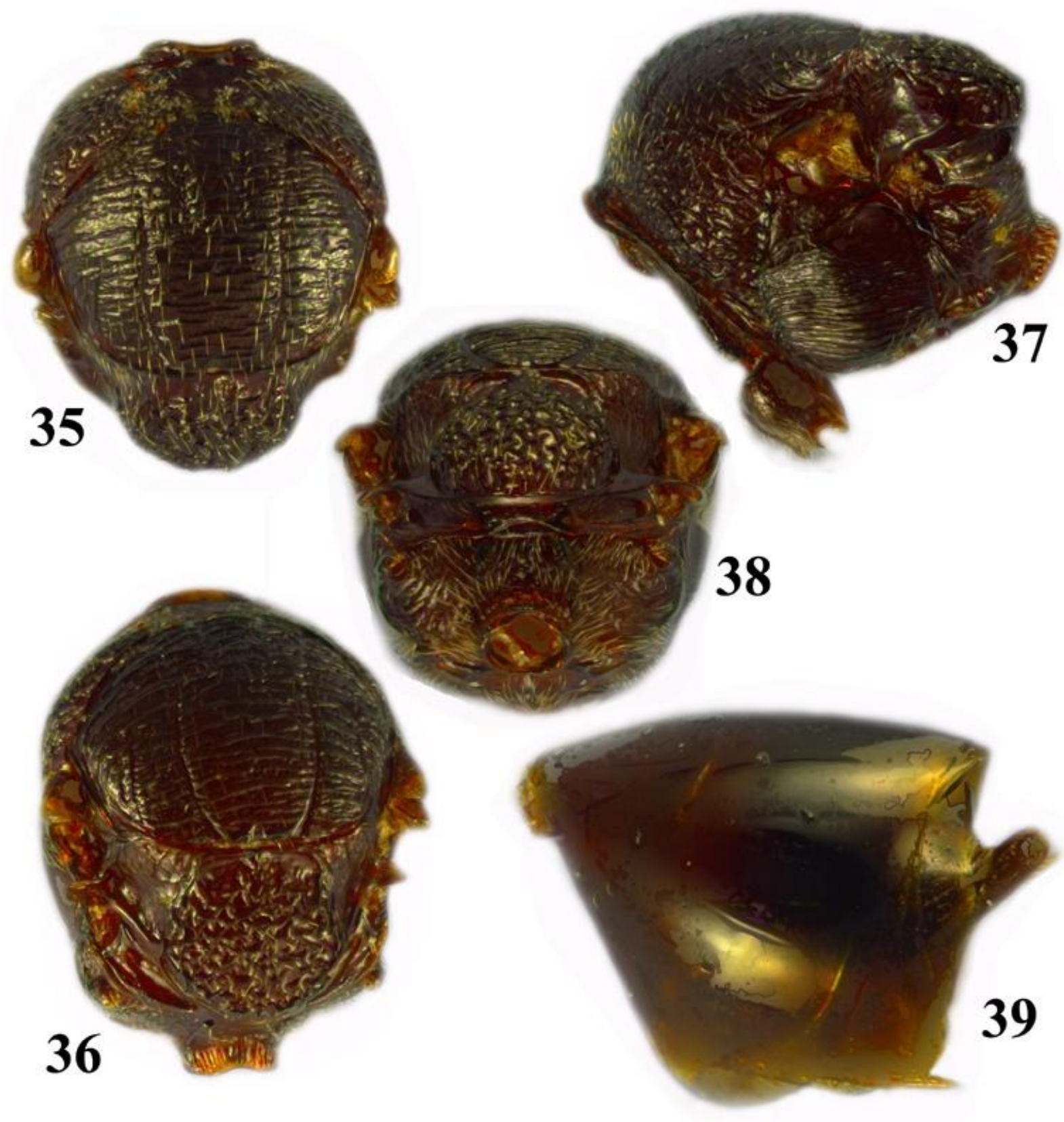

FIGURES A35-A39. Rhoophilus loewi, female: 35, mesosoma, anterodorsal view, 36, mesosoma, posterodorsal view, 37, mesosoma, lateral view, 38, metascutellum and propodeum, posterodorsal view, 39, metasoma, lateral view. 

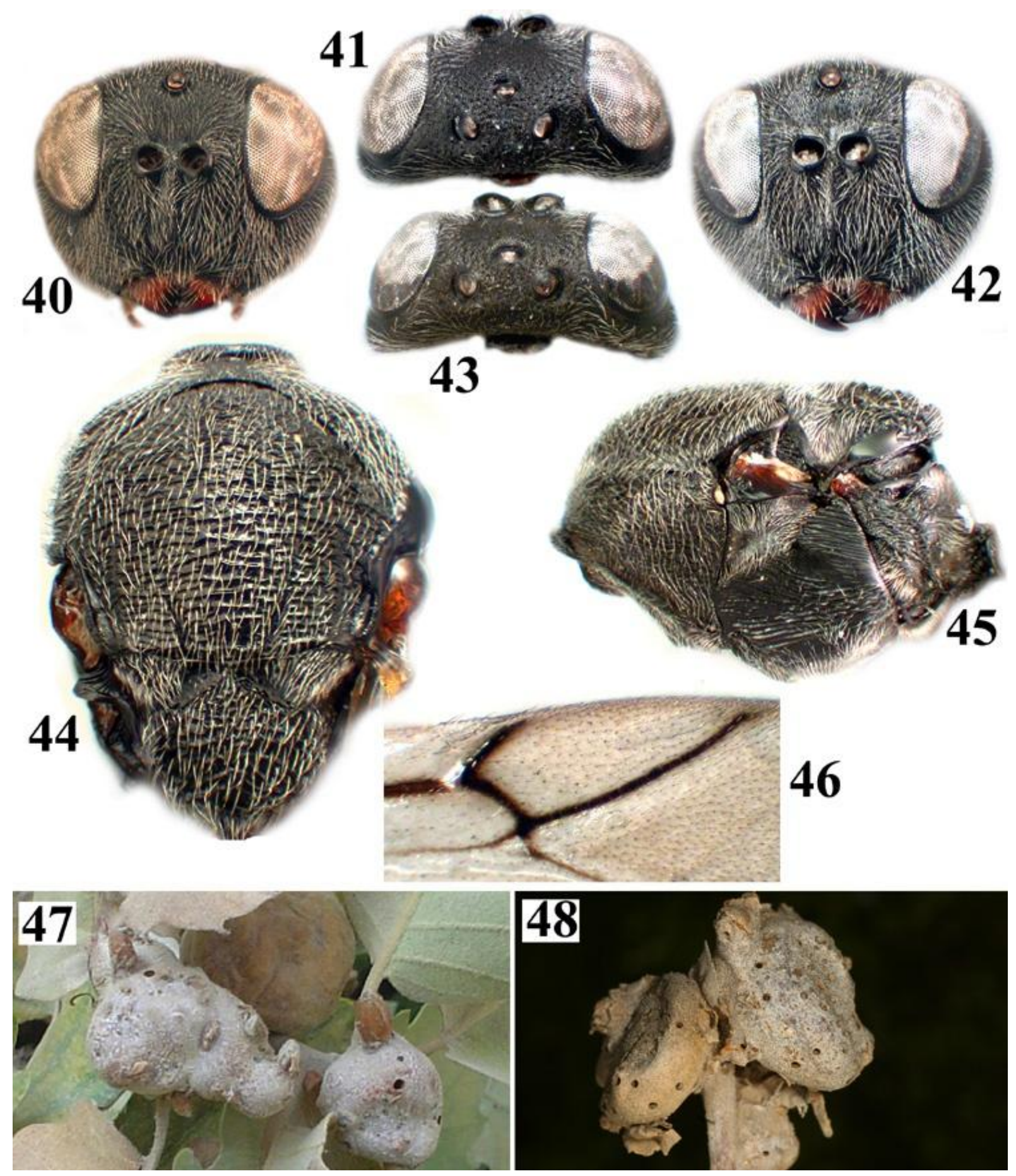

FIGURES A40-A48. Synophrus olivieri: 40-41, head, female: 40, frontal view, 41, dorsal view, 42-43, head, male: 42, frontal view, 43, dorsal view. 44-46, female: 44, mesosoma, dorsal view, 45 , mesosoma, lateral view, 46, fore wing, part. 47-48, gall (photos by M. Tavakoli). 


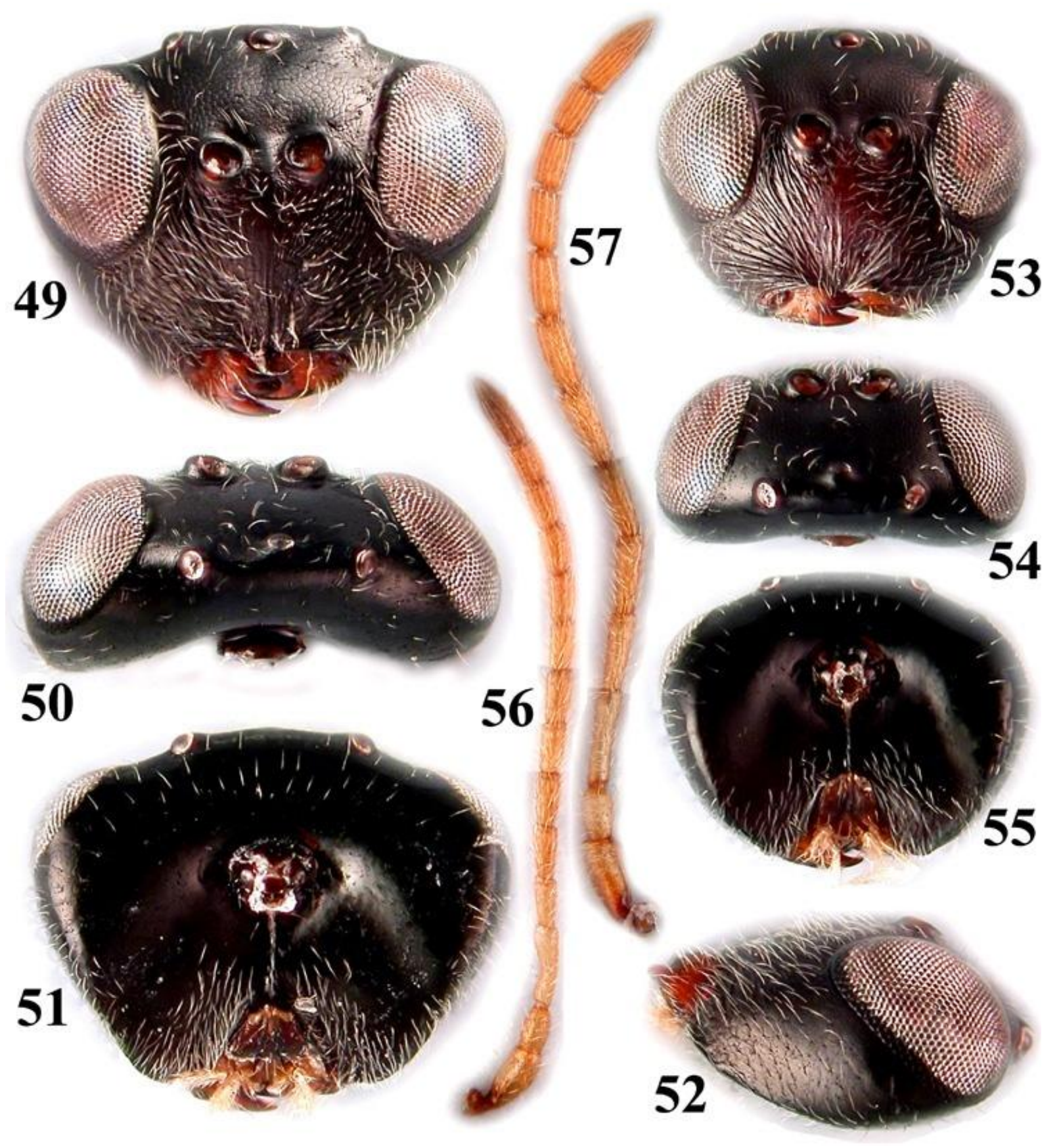

FIGURES A49-A57. Ufo cerroneuroteri: 49-52, head, female: 49, frontal view, 50, dorsal view, 51, posterior view, 52, lateral view. 53-55, head, male: 53, frontal view, 54, dorsal view, 55 , posterior view. 56-57, antenna: 56, female, 57, male. 

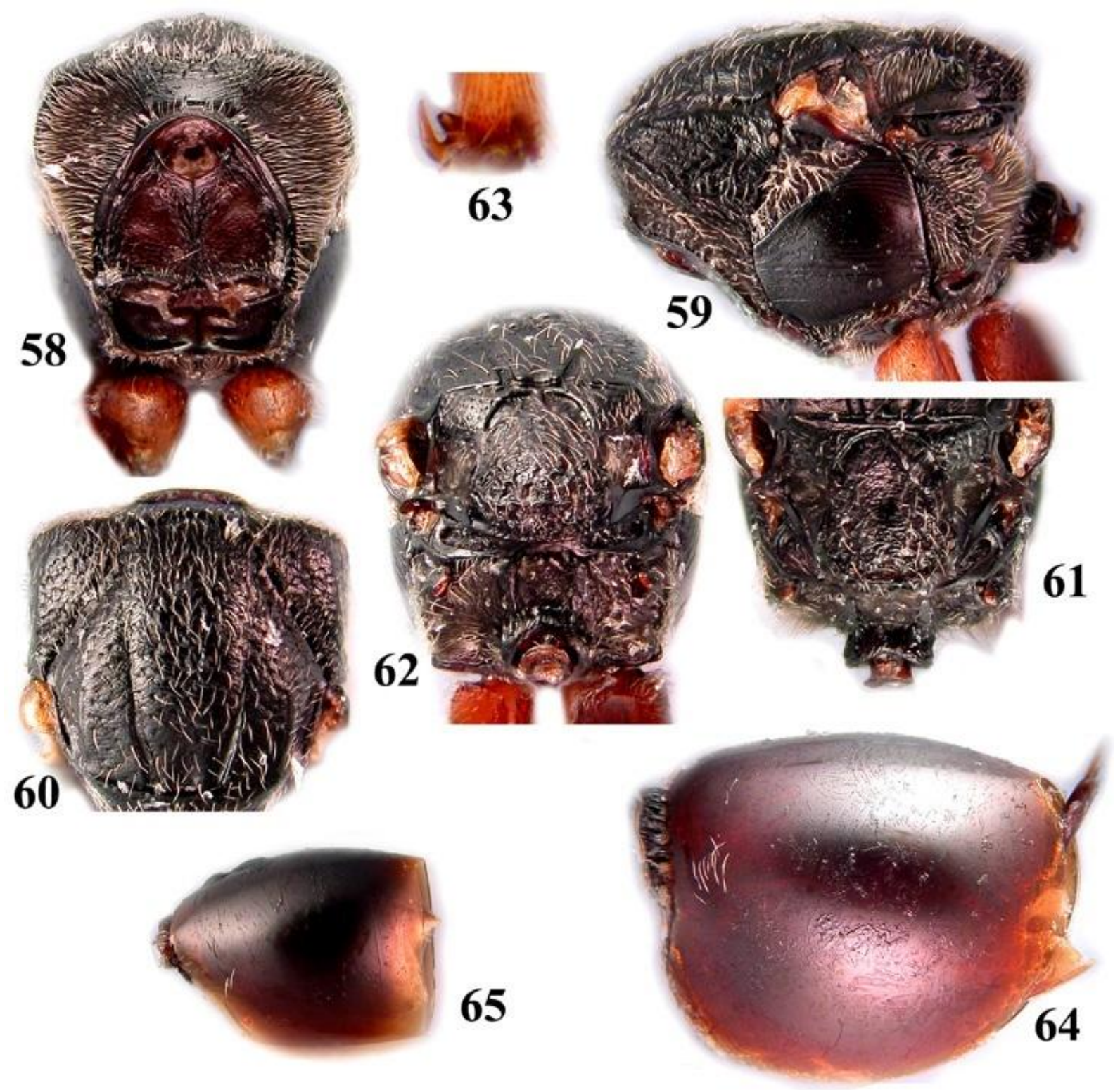

FIGURES A58-A65. Ufo cerroneuroteri: 58-66, female: 10, pronotum and propleuron, anterior view, 11, mesosoma, lateral view, 12, mesoscutum, dorsal view, 13, mesoscutellum, dorsal view, 14, metascutellum and propodeum, posterodorsal view, 15, tarsal claw, 16, metasoma, female, lateral view. 17, metasoma, male, lateral view. 

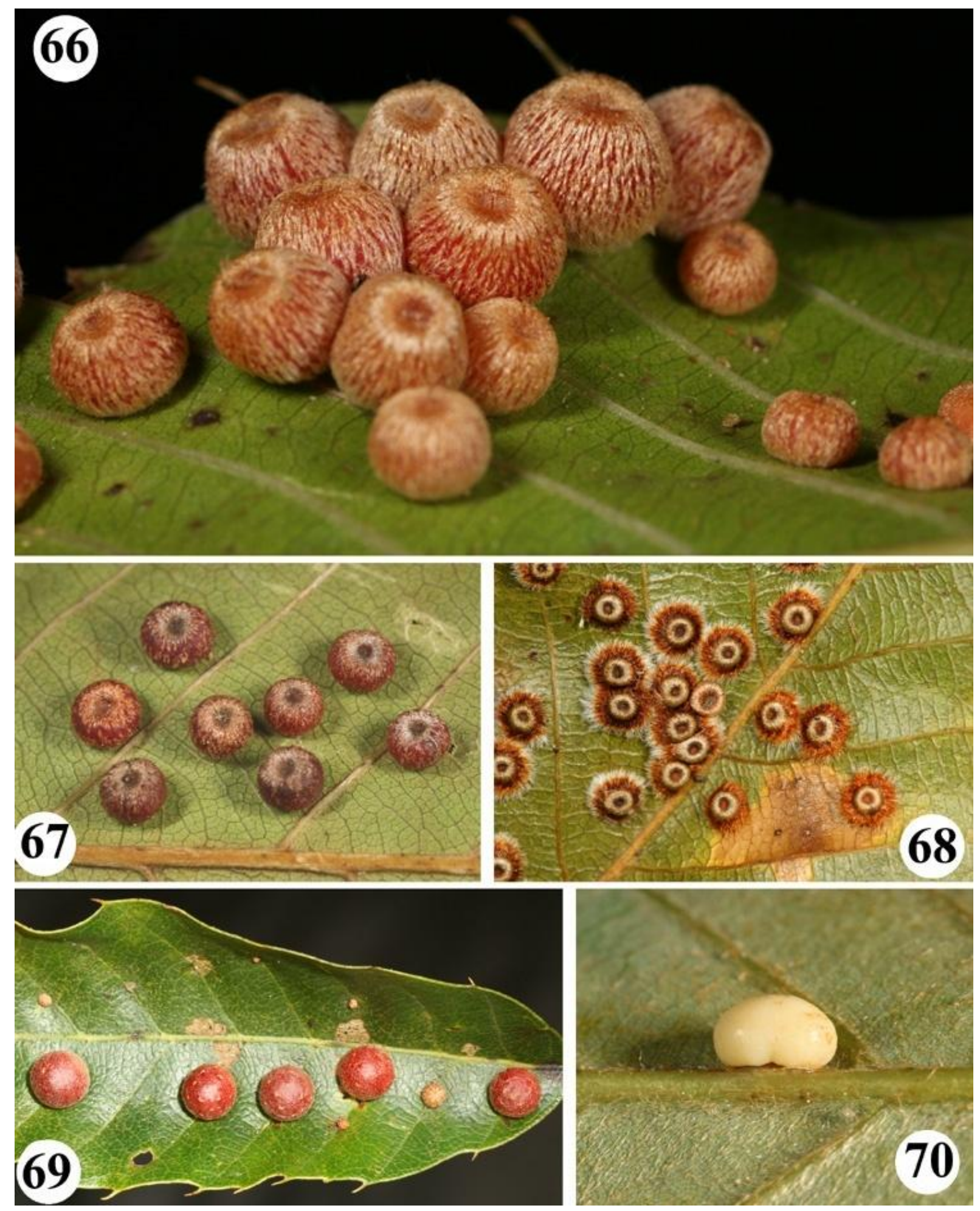

FIGURES A66-A70. Host oak galls of Ufo spp.: 66, Cerroneuroterus vonkuenburgi, asexual galls, 67, C. monzeni, asexual galls, 68, Latuspina nawai, asexual galls, 69, Trichagalma acutissimae, asexual galls, 70, Neuroterus hakonensis (= Andricus kunugifoliae), asexual galls (photos by G. Csóka). 


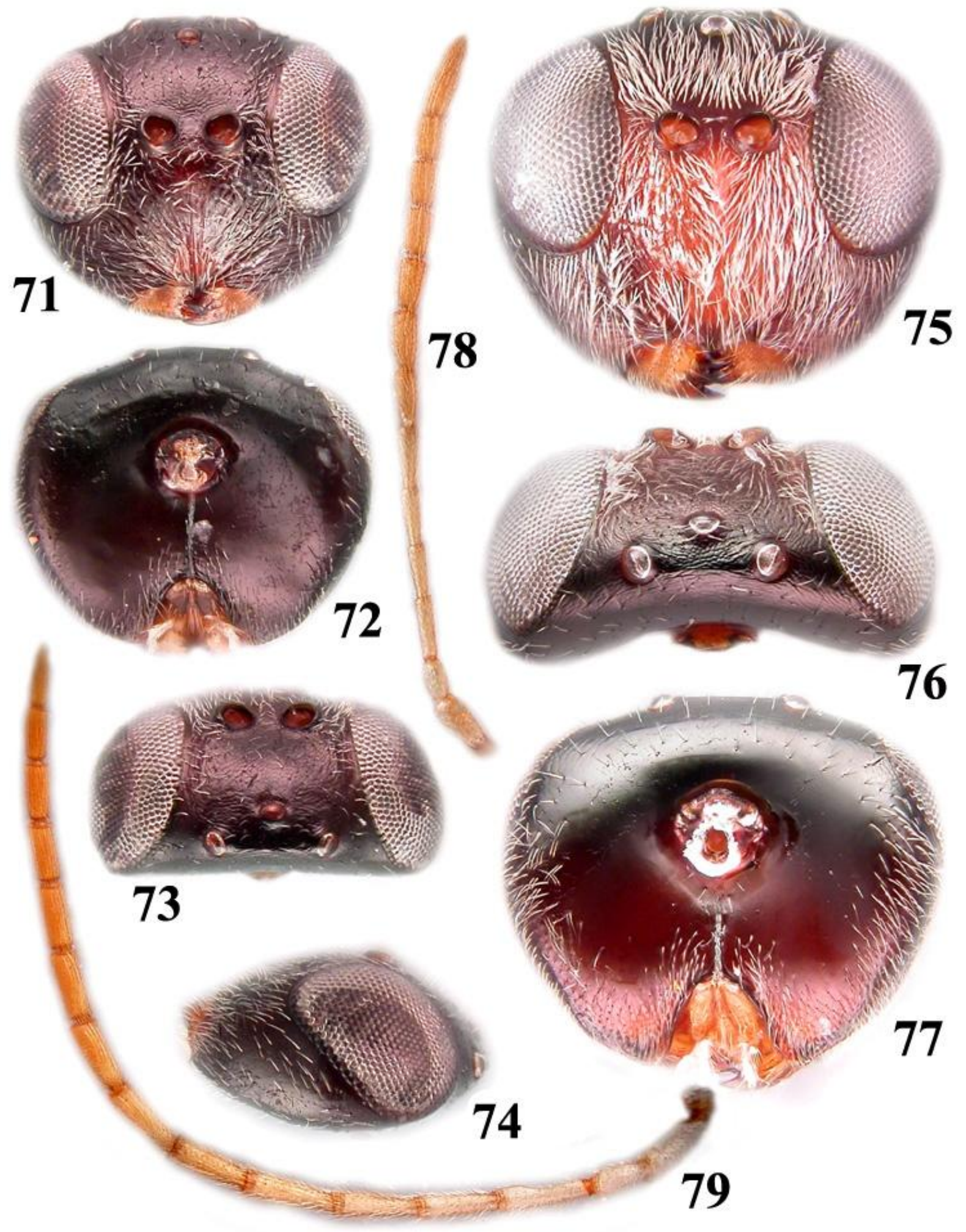

FIGURES A71-79. Saphonecrus lithocarpii: 71-74, head, female: 71, frontal view, 72, posterior view, 73, dorsal view, 74, lateral view. 75-77, head, male: 75, frontal view, 76, dorsal view, 77, posterior view. 78-79, antenna: 78, female, 79, male. 


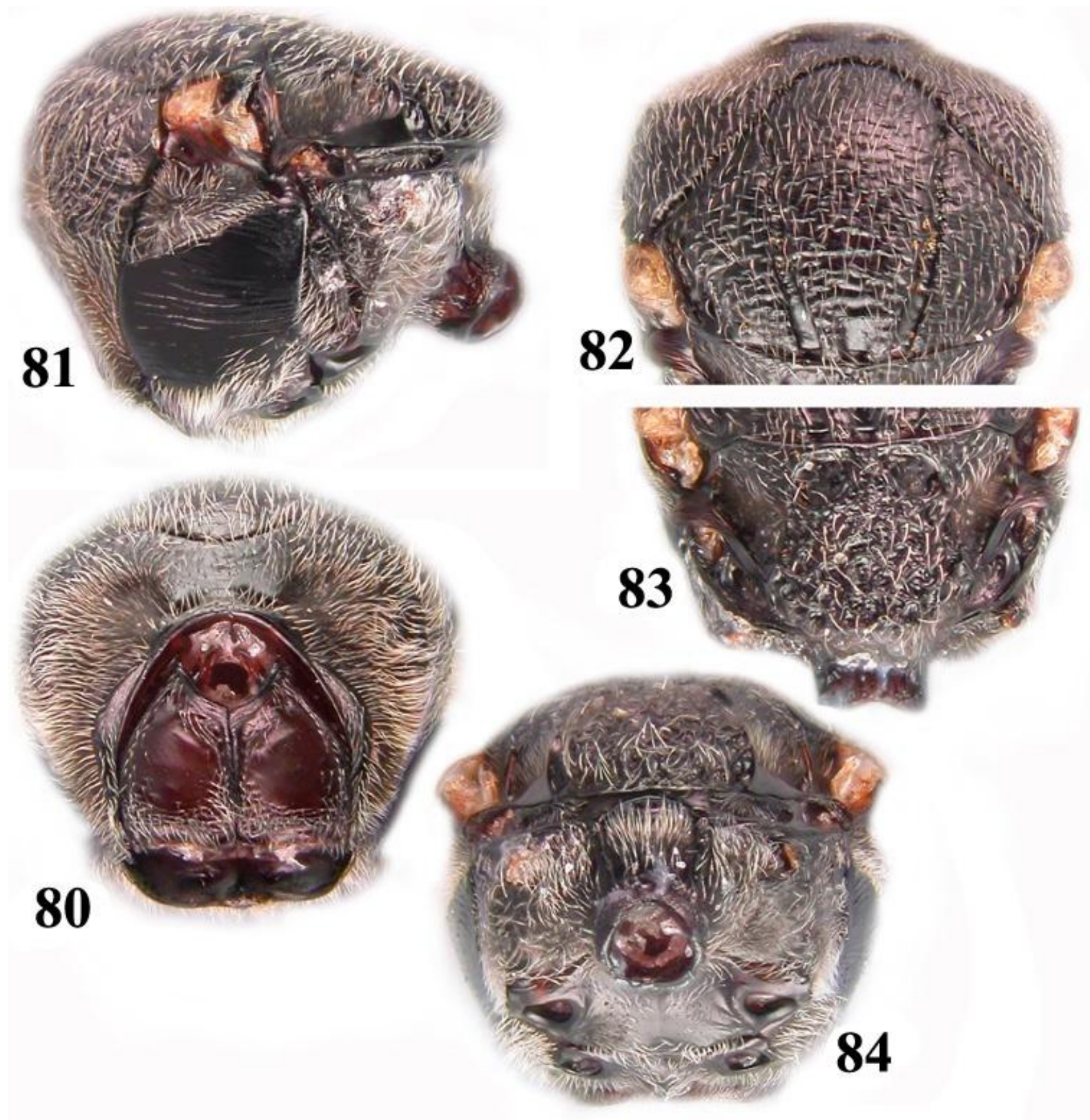

FIGURES A80-84. Saphonecrus lithocarpii, female: 80, pronotum and propleuron, anterior view, 81, mesosoma, lateral view, 82, mesoscutum, dorsal view, 83, mesoscutellum, dorsal view, 84 , metascutellum and propodeum, posterodorsal view. 

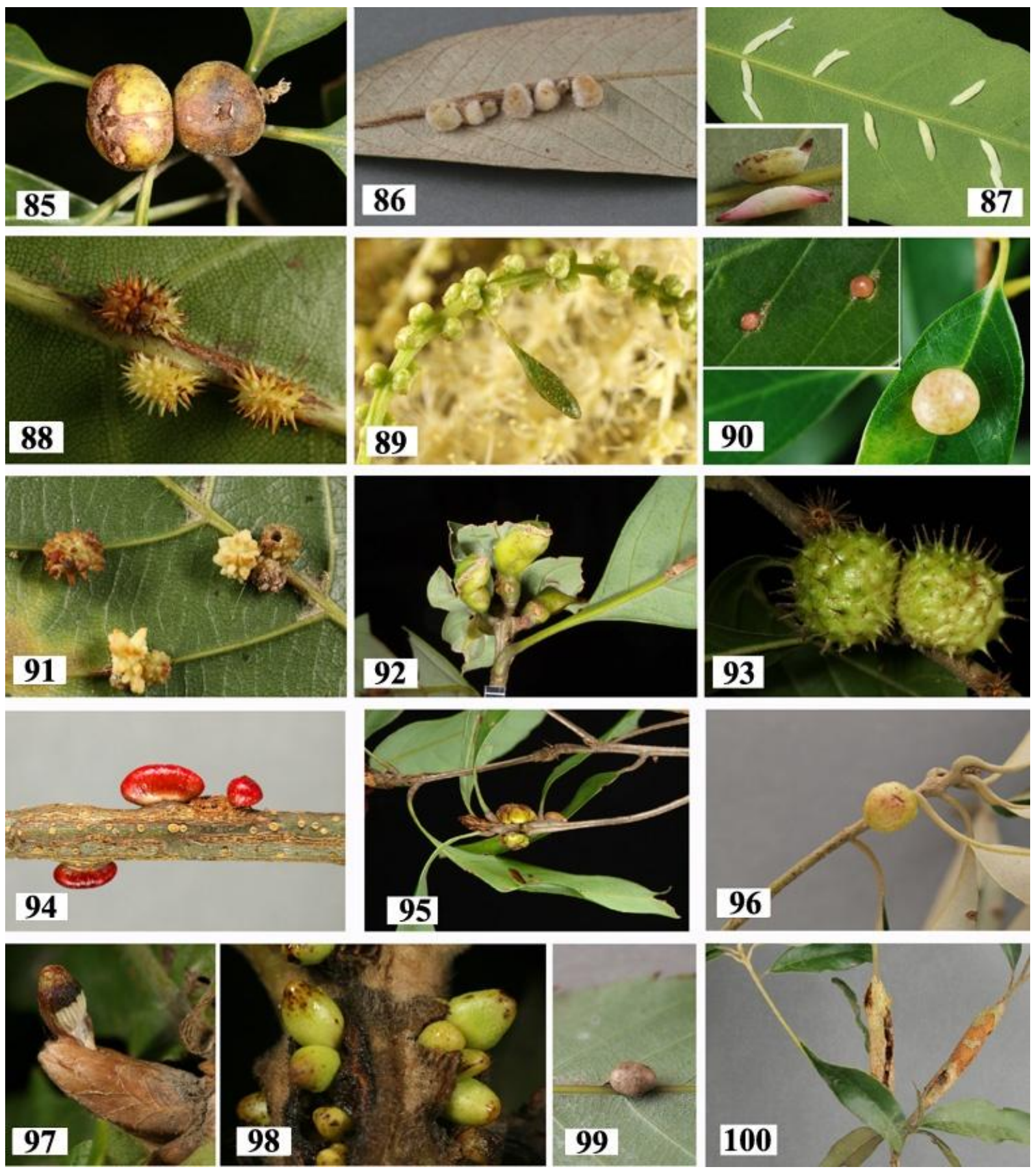

FIGURES A85-A100. Host galls of newly described Saphonecrus spp.: 85, undescribed bud gall, 86, undescribed leaf gall, 87, undescribed leaf gall, 88, undescribed leaf gall, 89, undescribed catkin gall, 90, undescribed leaf gall, 91, undescribed leaf gall, 92, undescribed leaf petiole thickening gall, 93, undescribed stem swelling-like gall, 94, undescribed stem swellinglike gall on $Q$. longinuxi, 95, undescribed stem swelling-like gall on Q. pachylomai, 96, undescribed rounded stem swelling-like gall, 97, undescribed bud gall, 98, asexual gall of Andricus hakonensis (=A.symbioticus), 99, undescribed egg-shaped gall on leaf midrib, 100, undescribed Dryocosmus multilocular stem swelling-like gall. 


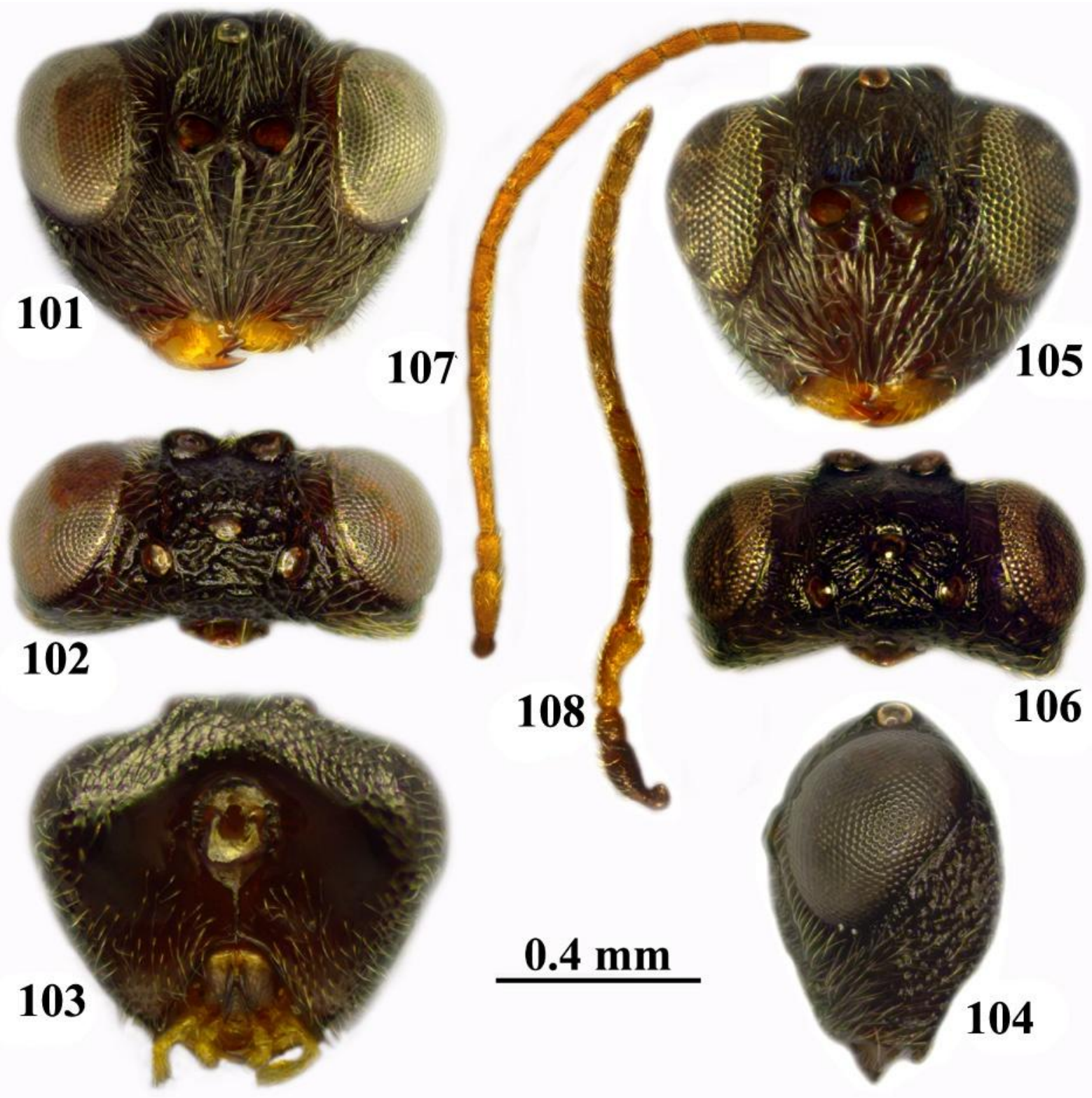

FIGURES A101-108. Synergus belizinellus: 101-104, head, female: 101, frontal view, 102 , dorsal view, 103, posterior view, 104, lateral view, 105-106, head, male: 105, frontal view, 106, dorsal view. 107-108, antenna: 107, female, 108, male. 

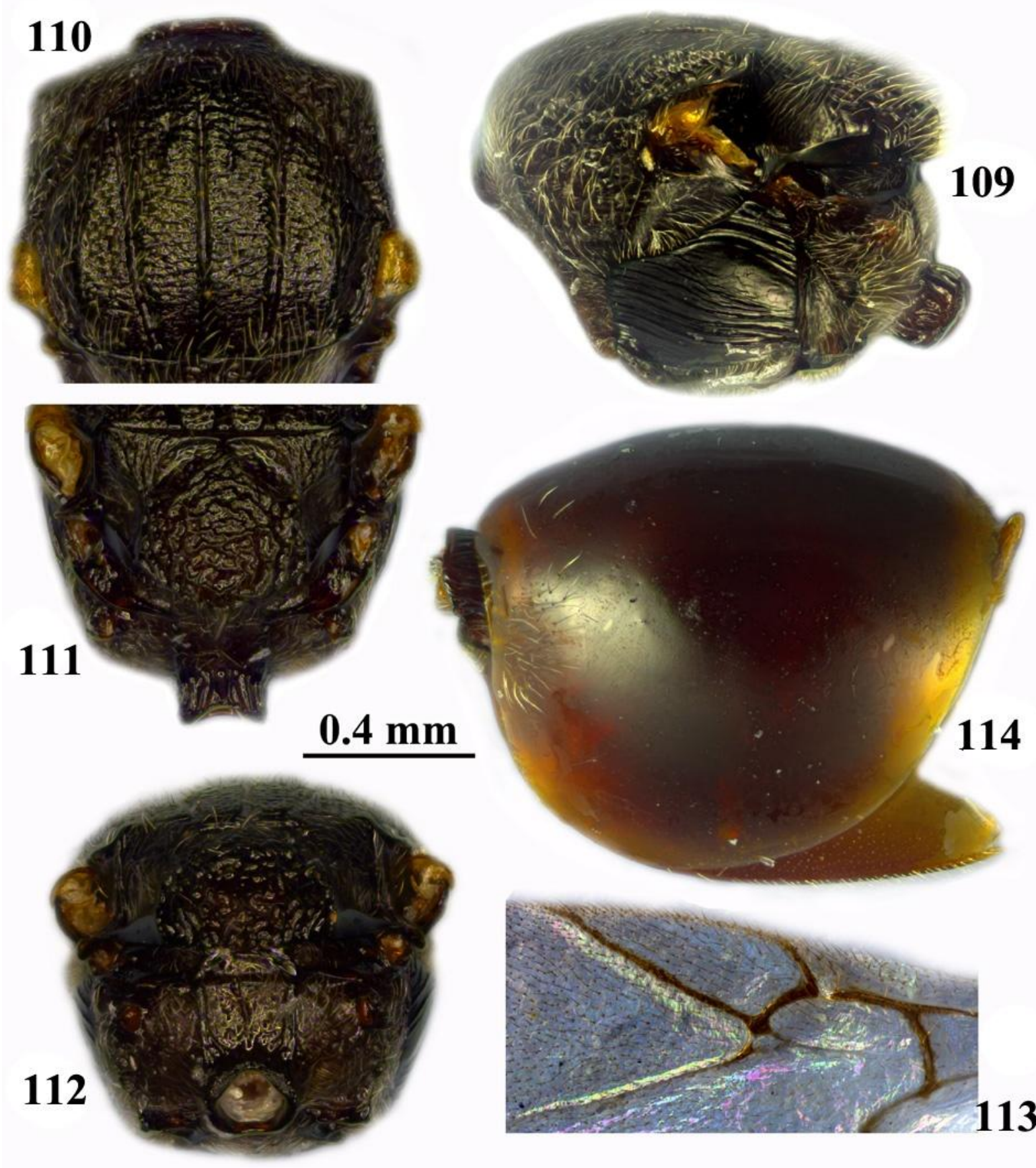

114
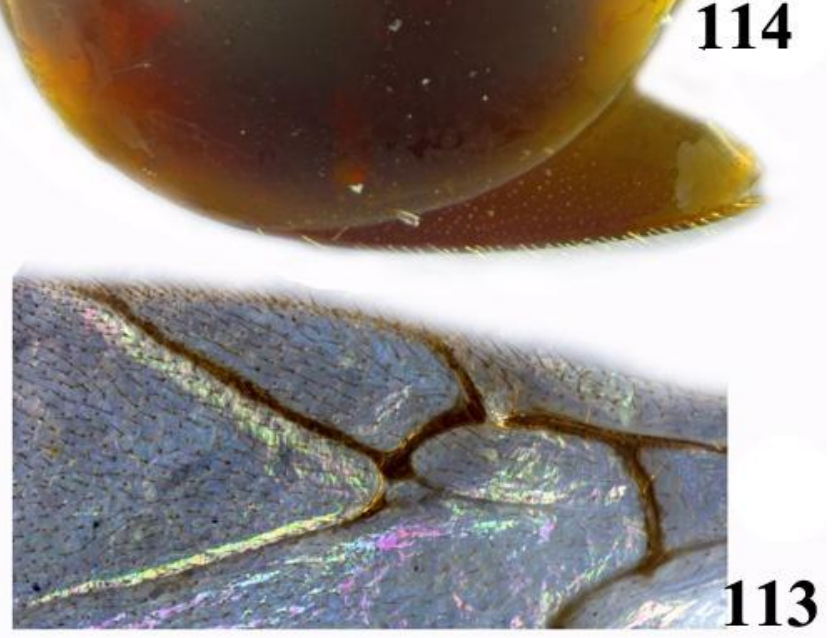

FIGURES A109-A114. Synergus belizinellus, female: 109, mesosoma, lateral view, 110, mesoscutum, dorsal view, 111, mesoscutellum, dorsal view, 112, metascutellum and propodeum, posterodorsal view, 113, fore wing, part, 114, metasoma, lateral view. 

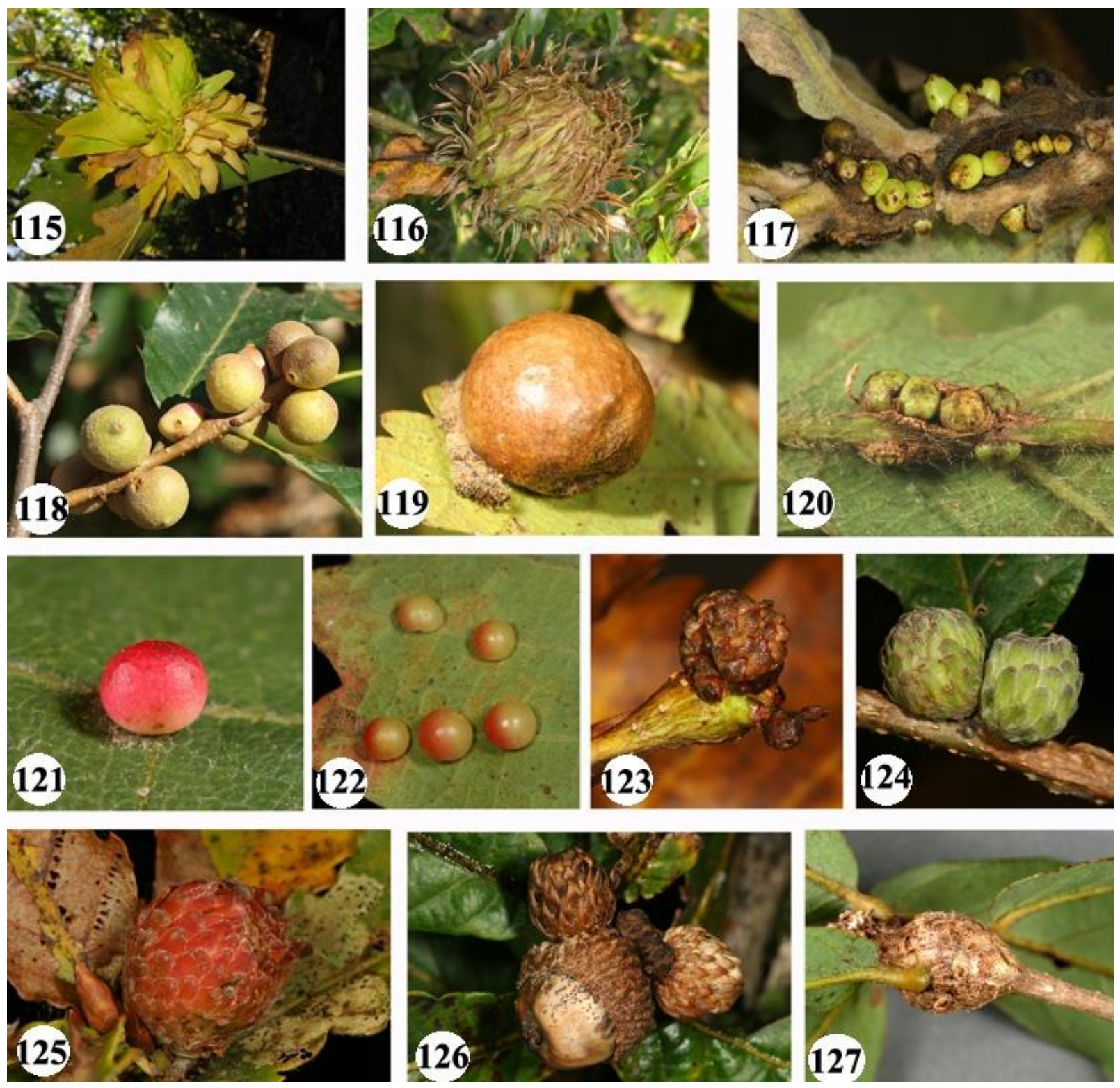

FIGURES A115-A127. Host oak galls of newly described Synergus spp.: 115, Andricus kashiwaphilus, asexual gall, 116, A. mukaigawae, asexual gall, 117, A. hakonensis (=A. symbioticus), asexual galls, 118, Trichagalma formosana, asexual galls, 119, Belizinella sp., asexual gall, 120, Ussuraspis sp., asexual gall, 121, unknown leaf gall, 122, unknown leaf gall, 123, unknown acorn gall, 124, unknown bud gall, 125, unknown bud gall, 126, unknown acorn gall (photos by G. Csóka). 127, unknown stem swelling-like gall (photos by Chang Ti Tang). 


\section{2. Annotated list of Cynipini of the Eastern Palearctic (Cynipoidea: Cynipidae)}

There are only few Cynipidae (Hymenoptera) reviews on the Eastern Palearctic species (Dalla Torre \& Kieffer 1910, Abe et al. 2007). Both are out of time and since then dozens of new species were described and a large number of nomenclatorial changes have been done. Recently some review papers were published on the Eastern Palearctic cynipid inquilines (Cynipidae: Synergini) (Pénzes et al. 2012) and its genera: Lithosaphonecrus Tang, Melika \& Bozsó (Bozsó et al. 2015), Saphonecrus Dalla Torre \& Kieffer (Schwéger et al. 2015b), Synergus Hartig (Schwéger et al. 2015b), and Ufo Melika \& Pujade-Villar (Melika et al. 2012). Abe et al. (2007) listed all the known Eastern Palearctic rose gallwasps (Diplolepidini) and herb gall wasps ("Aylacini"), while the list of oak gallwasps (Cynipini) currently is far from completeness.

Oak cynipid biology is in a period of rapid advance, both in terms of our understanding of cynipid biology and of resolution of taxonomic issues within the Cynipini. New genera, species and generations continue to be discovered in Japan (Ide et al. 2010, 2012, 2013, 2016, Wachi \& Abe 2010), Taiwan (Melika et al. 2010, 2011, 2012, 2013, Tang et al. 2009, 2011a, b, 2012a, b, 2016a, b, c), China (Pujade-Villar \& Wang 2012, Wang et al. 2013a, b, Abe et al. 2014a, Pujade-Villar et al. 2014), Vietnam (Abe et al. 2014b). In particular, Taiwan and China are areas with high oak (Quercus L.) and oak relatives' (Castanea Miller, Castanopsis Miller, Lithocarpus Blume) species richness whose cynipid faunas remain little-studied, and future work will certainly reveal further new species.

This list summarizes what is known of the biology and taxonomic history of each oak gallwasp species and generation. We have had to address two difficult issues - the set of recognized species and lifecycles (discussed further below), and the sources of information used. The information is drawn from our own surveys and from selected published literature. Literature sources are given where appropriate, but we have not attempted to provide exhaustive literature support for data on common and widespread species. Where possible we cite regional reviews (e.g. Kovalev (1965) for Far East Russia, Monzen $(1953,1954)$ for Japan). The Eastern Palearctic as here defined includes Asia east to Iran, the Ciscaucasus (Transcaucasus) and the Ural Mountains. Species known only from more western regions, such as from Iran, the Transcaucasus, Dagestan are not included.

Genera and species are given in alphabetical order.

\section{Andricus Hartig, 1840}

\section{Andricus deqingis Wang, Guo \& Chen, 2013}

Lifecycle. Only the sexual generation is known (Wang, Guo \& Chen 2013).

Geographic distribution. China: Zhejiang (Wang, Guo \& Chen 2013).

Host plants. Quercus sp. (Wang, Guo \& Chen 2013).

Gall location and structure. Galls on branches and twigs, $1.0-2.5 \mathrm{~mm}$ long, yellowish, usually in clusters, each gall contains single thin-walled larval chamber (Wang, Guo \& Chen 2013).

Phenology. Adults emerge in May (Wang, Guo \& Chen 2013).

Andricus flavus Pujade-Villar, Wang, Guo \& Chen, 2014

Lifecycle. Only the sexual generation is known (Pujade-Villar et al. 2014).

Geographic distribution. China: Zhejiang (Pujade-Villar et al. 2014).

Host plants. Quercus fabrei Hance (Pujade-Villar et al. 2014). 
Gall location and structure. Multilocular leaf galls. Individual galls are nearly spherical, usually solely located on the underside of leaves. The young gall is juicy and freshly green, the surface is covered with small raised tubercles. The gall is brown or grey with a tough woody wall, 2.0-3.0 mm in diameter; turns dry and dark brown when mature, contains 4-5 larval chambers, each $0.5-0.7 \mathrm{~mm}$ in diameter (Pujade-Villar et al. 2014) .

Phenology. Galls appear on the host plant from early April; mature in early May; adults emerge in late May (Pujade-Villar et al. 2014).

Similar galls. The galls somehow resembles those of Andricus xishuangbannaus (Tang et al. 2012b) and A. pseudocurvator (Tang et al. 2011b).

\section{Andricus formosanus Tang \& Melika, 2009}

Lifecycle. Only the sexual generation is known (Tang et al. 2009).

Geographic distribution. Taiwan (Pingtung County), the only natural stands of $Q$. dentata in Taiwan (Lu et al. 2006). It is possible that this species also occurs in continental China, as well as in Japan and the Russian Far East, where Q. dentata is a common species.

Host plants. Quercus dentata Thunb. (Tang et al. 2009).

Gall location and structure. Aggregated integral leaf galls cause swelling on both, upper and lower surfaces of the leaf. Individual galls are approximately spherical, 3.5-5.0 $\mathrm{mm}$ in height, each contains a single (unilocular) thin-walled larval chamber, 1.5-1.7 $\mathrm{mm}$ in diameter, attached by a tiny filament to the outer wall of the gall. In mature galls, the larval chamber loses its connection to the gall wall, and rolls freely within an internal air space. Though galls can occur separately on a leaf, they are often aggregated into masses $20-70 \mathrm{~mm}$ long that distort the leaf blade. Young galls are fleshy and pale green to yellowish, remaining soft when mature (Figs A128-129) (Tang et al. 2009).

Phenology. Galls mature in March; adults emerge in late March (Tang et al. 2009).

Similar galls. Andricus formosanus most closely resembles the sexual generation of an Eastern Palearctic species, Andricus moriokae Monzen which induces integral leaf galls on $Q$. dentata and Q. serrata Thunb. (= Q. glandulifera Blume) and is known from Japan and the Russian Far East (Monzen 1953, Kovalev 1965). Although the galls induced by the two species are structurally similar, in A. formosanus the gall clusters always much bigger, comprising many galls, often merged together, while in A. moriokae only a few galls are typically found on each leaf.

Andricus hakonensis (Ashmead, 1904)

Synonyms: Callirhytis hakonensis Ashmead, 1904; Andricus attractus Kovalev, 1965 (Wachi \& Abe 2010); Andricus symbioticus Kovalev, 1965 (Wachi \& Abe 2010); Andricus oblongus Monzen, 1953 (Yukawa \& Masuda 1996). Andricus hakonensis (Ashmead, 1904) comb. nova in Wachi \& Abe (2010).

Lifecycle. Alternating sexual and asexual generations are known (Wachi \& Abe 2010).

Geographic distribution. Japan (Hokkaido, Honshu, Shikoku, Kyushu), Russian Far East (Lake Khasan in Primorie), Korea (Kovalev 1965, Yukawa \& Masuda 1996, Katsuda \& Yukawa 2004, Abe 1988, 1992, Wachi \& Abe 2010).

Host plants. Quercus aliena Blume, Q. dentata, Q. mongolica Fisch. ex Ledeb., Q. serrata (Kovalev 1965, Abe 1992, Katsuda \& Yukawa 2004).

Gall location and structure. Asexual generation bark galls are rounded-oblong, smooth, 7-8 mm high; green or greenish-raspberry, usually clustered on twig with a single larval cell (Fig. 
A98, A117). The gall is secreting honeydew-like sticky substance which attracts ants and protect galls against parasitoid attack (Kovalev 1965, Abe 1988, 1992). Sexual generation galls are irregular spherical swelling of leaf vein and petiole; the immature gall is partially red, the mature one is yellowish green to green; with several, ovoid larval cells in the gall (Fig. A130) (Wachi \& Abe 2010).

Phenology. The female adult of the asexual generation oviposits into buds before the bud burst, in early spring. The gall of the sexual generation appears on the leaf petiole or midrib in spring; adults emerge in early summer. Asexual galls appear on the twigs in late summer to autumn; adults emerge in early spring of the following year or even one year later (Yukawa \& Masuda 1996).

Similar galls. The asexual generation galls are similar to those of the asexual generation of the western-palearctic species Andricus sieboldi (Hartig, 1843) [erroneously mentioned by Kovalev (1965) as A. testaceipes Hartig, 1840]. However, in contrast to A. sieboldi, the growing asexual galls of $A$. hakonensis produce honeydew and attract ants; galls fall when they mature, in late summer (Kovalev 1965). Asexual galls cluster encircling young sprouts, and are very similar to those of the asexual generation of western-palearctic Dryocosmus cerriphilus Giraud, 1859, which also produce honeydew and attract ants (Abe 1988, 1992).

Comments. Andricus oblongus Monzen, 1953 was described based on females only, which induce gall masses on the bark of Q. serrata in Japan (Monzen 1953). In the Monzen collection (Kyoto University) 11 pins with adults were found, all of which belong to an inquiline Synergus species; also the original description is a Synergus description (Monzen (1953). Thus, the name cannot be used for a gallwasp. According Yukawa \& Masuda (1996) what Monzen (1953) described as A. oblongus is an Andricus symbioticus gall attacked by inquilines.

Andricus kashiwaphilus Abe, 1998

Lifecycle. Alternating sexual and asexual generations are known (Abe 1998).

Geographical distribution. Japan (Hokkaido, Chugoku, Kyushu) (Abe 1998), Russian Far East (Schwéger et al. 2015a, b), China (Pujade-Villar et al. 2016).

Host plants. Quercus dentata (Abe et al. 1998, 2007, Melika 2012).

Gall location and structure. The flower-shaped asexual gall is single or sometimes a few galls clustered, in shape similar to flower of rose, surrounded by compact leaf clusters, developed from axillary bud; the single larval cell is oval, 3.5-4.0 $\mathrm{mm}$ in diameter and $6.0 \mathrm{~mm}$ high (Fig. A115). Sexual galls are integral leaf galls, usually located on the leaf midrib (Abe 1998).

Phenology. Life cycle is very similar to that of A. mukaigawae (Wachi et al. 2012).

Comments. The morphology of adults similar to that of $A$. mukaigawae and thus it is complicate to differentiate the two species based on their morphology only (Abe et al. 2007).

\section{Andricus marmoratus Kovalev, 1965}

Lifecycle. Only the sexual generation is known (Kovalev 1965).

Geographic distribution. Russian Far East (Kovalev 1965).

Host plants. Quercus mongolica (Kovalev 1965, Melika 2012).

Gall location and structure. Tiny unilocular integral spherical leaf galls, 1-2 mm in diameter; green when growing, turn brown when mature (Kovalev 1965).

Phenology. Adults emerge in May (Kovalev 1965).

Comments. This species is closely resembles A. moriokae (Kovalev 1965, Abe et al. 2007). 
Andricus mairei (Kieffer, 1906)

Synonyms: Originally was described in Parandricus Kieffer, 1906, a genus synonymized to Andricus (Melika \& Abrahamson 2002).

Lifecycle. Only the sexual generation is known (Kieffer 1906).

Geographic distribution. China (Hunan Province) (Kieffer 1906, Yang et al. 2012).

Host plants. Quercus serrata (= Q. glandulifera var. brevipetiolata Nakai) (Yang et al. 2012).

Gall location and structure. Catkin galls that forms clusters of up to 30 galls in one catkin (Abe et al. 2007, Yang et al. 2012).

Andricus mesostegius Kovalev, 1965

Lifecycle. Only the sexual generation is known. The species was described on the basis of males only (Kovalev 1965).

Geographic distribution. Russia (Far East) (Kovalev 1965, Melika 2012).

Host plants. Quercus dentata (Kovalev 1965).

Gall location and structure. The gall is unknown; probably inconspicuous bud galls (Melika 2012).

Andricus moriokae Monzen, 1953

Lifecycle. Alternating sexual and asexual generations are known. The sexual generation was described by Monzen (1953) while the asexual generation was found later (Katsuda \& Yukawa 2003, Abe et al. 2007).

Geographic distribution. Japan and Russia (Far East) (Monzen 1953, Kovalev 1965).

Host plants. The sexual generation galls on Quercus dentata and Q. serrata (Monzen 1953, Kovalev 1965); the asexual generation galls on Quercus serrata (Katsuda \& Yukawa 2003, Abe et al. 2007).

Gall location and structure. The sexual generation induces integral leaf galls, which produce from both sides of the leaf, 2.0-2.5 $\mathrm{mm}$ in diameter (Monzen 1953); the asexual generation induces bud galls (Katsuda \& Yukawa 2003).

Phenology. The sexual adults emerge in May (Monzen 1953).

Andricus mukaigawae (Mukaigawa, 1913)

Synonyms: Dryophanta mukaigawae Mukaigawa, 1913; Andricus japonicus Ashmead, 1904 (in Monzen 1931); Andricus mukaigawae: comb. nova in Abe (1986).

Lifecycle. Alternating asexual and sexual generations are known (Abe 1986).

Geographical distribution. Japan and China (Abe 1986, 2007, Pujade-Villar et al. 2016), Russia (Far East) (Kovalev 1965), probably North Korea (Kovalev 1965), South Korea (authors), India (West Kameng District) (Abe et al. 2012).

Host plants. Quercus aliena, Q. dentata, Q. griffithii Hook. F \& Thomson ex Miq., $Q$. mongolica var. grasseserrata Rehid. et Wils., Q. serrata (Abe 1986, 2007, Wachi et al. 2012).

Gall location and structure. A single, bud urn-shaped gall, surrounded by numerous lanceolate spines, developed from axillary bud; maximum diameter $25 \mathrm{~mm}$; single larval cell oval, embedded in an acorn-like cup, 3.5-4.0 mm in diameter and $6.0 \mathrm{~mm}$ high (Fig. A116). The sexual gall is a leaf gall, single ovoid, with a thin wall, produced on edge or midrib of leaf, inhibiting of development of surrounding leaf tissue; maximum diameter 2-3 mm (Abe 1986).

Phenology. The sexual generation galls appear on axillary buds in early June; mature in August; adults emerge in early December to early February. The sexual generation galls appear on the 
edges and midribs of leaves in early April, when leaf buds begin to break; adults emerge in late April. In late April and early May, females deposit their eggs in or on leaf primordia of immature axillary buds (Abe 1986). This species was studied in detail by Abe (1986, 1988, 1991, 1998, 2007, Wachi et al. (2012).

Similar galls. Galls resemble those of Andricus targionii which, however, are usually smaller in size (Abe 1986).

Andricus pseudocurvator Tang \& Melika, 2011

Lifecycle. Only the sexual generation is known (Tang et al. 2011b).

Geographic distribution. Taiwan (Nantou and Taichung Counties). It is possible that this species also occur in continental China and Japan, where Q. serrata occurs (Tang et al. 2011b).

Host plants. Quercus serrata (Tang et al. 2011b).

Gall location and structure. Integral leaf galls. Individual galls approximately spherical, 2.4$3.6 \mathrm{~mm}$ in height, and integral to leaf lamina. Galls appear as yellowish or brownish dots on leaves, cause swelling on both upper and lower surfaces of leaf, each contains single thin-walled larval chamber, $1.6-2.3 \mathrm{~mm}$ in diameter. Outer wall of larval chamber attached to inner wall of surrounding gall tissue in both growing and mature galls. Galls usually occur separately on leaf and do not aggregate together (Figs A131-132). Young galls are fleshy, yellowish to brownish, remaining soft as they mature (Tang et al.2011b)

Phenology. Galls mature in early March; adults emerge in mid and late March (Tang et al. 2011b).

Similar galls. The shape, structure and location of the gall is very similar to that induced by a Western Palearctic species, Andricus curvator Hartig, thus the species was named as "curvatorlike" - pseudocurvator (Tang et al. 2011b).

Andricus pseudoflos (Monzen, 1954)

Synonyms: Cynips pseudoflos Monzen, 1954; Adleria pseudoflos (Monzen, 1954) (comb. nova in Kovalev (1965)). Judging from the life cycle, Abe (1986) considered that this species is synonymous with A. targionii. Later, Abe (2007) regarded this species as a distinct species based on cytological data and host plant exploitation, and transferred it into the genus Andricus (Monzen 1954).

Lifecycle. Only the asexual generation is known (Monzen 1954). The biology of this species was studied in detail by Abe (1986), Weih (1965), Wachi et al. (2012).

Geographic distribution. Japan, Korea, China and Russian Far East (Primorie) (Monzen 1954, Kovalev 1965, Weih 1965, Abe 1986, 2007, Abe et al. 2007).

Host plants. Quercus dentata (Monzen 1954, Kovalev 1965).

Gall location and structure. The gall is a flower-like deformation on the top of the twig or sometimes the midrib of the leaf (Fig. A133). The leaflet length is $40-60 \mathrm{~mm}$, width $15 \mathrm{~mm}$. The larval chamber oblong, length $6.0 \mathrm{~mm}$, width $3.0 \mathrm{~mm}$, side wall thin, smooth, yellowish (Monzen 1954).

Phenology. Galls begin to produce late in May, green and turn to yellowish brown in October; adults over-winter in galls and emerge in April or May next year (Monzen 1954).

\section{Andricus quercicola Shinji, 1940}

Lifecycle. Only the sexual generation is known (females were described only) (Monzen 1953).

Geographic distribution. Japan (Monzen 1953). 
Host plants. Quercus dentata and Q. serrata (Monzen 1953).

Gall location and structure. The leaf swelling gall is rounded, yellowish, $3 \mathrm{~mm}$ in diameter. It is on the underside of the leaf, integral, unilocular. The larval chamber is in the tissue of the leaf blade (Monzen 1953).

Phenology. Adults emerge in late June of the same year when the gall was formed (Monzen 1953).

\section{Andricus songshui Tang \& Melika, 2011}

Lifecycle. Only the sexual generation is known (Tang et al. 2011b).

Geographic distribution. Taiwan (Nantou County). It is possible that this species is also distributed in continental China and Japan where Q. serrata occurs (Tang et al. 2011b).

Host plants. Quercus serrata (Tang et al. 2011b).

Gall location and structure. Integral leaf galls, usually at the base of a leaf midrib. The swelling, on both unfolded young leaves and base of midrib, is 1.8-2.5 mm long (Fig. A134). Galls are usually yellowish and single; galls on unopened young leaves are green and in clusters of 1-3, multilocular (Figs A135-136). The two gall morphotypes can be found in the same month (Tang et al. 2011b).

Phenology. Galls mature in early March; adults emerge in mid to late March (Tang et al. 2011b).

\section{Andricus targionii Kieffer, 1903}

Synonyms: Cynips pseudoflos Monzen, 1954 (erroneously synonymised by Abe (1986)); Adleria sakagamii Kovalev, 1965 (synonymised by Abe (1986)).

Lifecycle. Only the asexual generation is known (Abe 1986, 2007, Weih 1965).

Geographical distribution. Japan, South Korea, China, Far East Russia (Kieffer 1903, Dalla Torre \& Kieffer 1910, Kovalev 1965, Abe 1986, Abe et al. 2007)

Host plants. Quercus aliena, Q. wutaishanica Mayr (= Q. liaotungensis Koidz.) (Abe 1986, 2007, Abe et al. 2007).

Gall location and structure. The asexual bur shaped galls are on midribs, leaf margins, petioles and elongating shoots, usually clustered (Abe 2007).

Phenology. Galls mature in late summer; adults usually emerge from late October to late November (Abe 1986).

Similar galls. Andricus mukaigawae galls are similar, however, always bigger than galls of $A$. targionii (Abe 1986).

\section{Andricus xishuangbannaus Melika \& Tang, 2012}

Lifecycle. Only the sexual generation is known (Tang et al. 2012b).

Geographic distribution. China (Xishuangbanna, Yunnan and Zhejiang Province) (Tang et al. 2012b, Wang et al. 2013a).

Host plants. Quercus griffithii (Tang et al. 2012b), Q. serrata (Wang et al. 2013a).

Gall location and structure. Integral blister leaf galls, always singular, never in clusters. The mature gall contains outer gall tissues and an inner, hollow-like larval chamber. The gall is approximately spherical, $2.4-3.6 \mathrm{~mm}$ in height, and the outer gall tissues protrude equally on both sides of leaf lamina (Figs A137-138). Outer wall of larval chamber attaches to the inner wall of surrounding gall tissues. Young gall is fleshy, yellowish to brownish, turning dry and dark brown when mature (Tang et al. 2012b). 
Phenology. Galls mature in mid-April; adults emerge approximately one week later (Tang et al. 2012b).

Similar galls. Most closely resembles galls of $A$. pseudocurvator, however, galls of $A$. xishuangbannaus hollow-like, without separated larval chambers inside, whereas the galls of $A$. pseudocurvator are with free-rolling larval chambers inside (Tang et al. 2012b).

\section{Belizinella Kovalev, 1965}

Belizinella gibbera Kovalev, 1965

Synonyms. Belizinella gibbera Kovalev, 1965. Trigonaspis gibbera: Melika \& Abrahamson (2002); Belizinella gibbera Kovalev, 1965, comb rev. in Melika (2012).

Lifecycle. Only the asexual generation is known. Females are wingless (Kovalev 1965, Melika 2012).

Geographic distribution. Russian Far East (Kovalev 1965).

Host plants. Quercus dentata (Kovalev 1965).

Gall location and structure. Detachable spherical leaf galls on the underside of the leaves, with glabrous surface, 10-12 mm in diameter, unilocular (Fig. A139), with a larval chamber in the center of the gall, $2.5 \mathrm{~mm}$ in diameter (Kovalev 1965).

Phenology. Adults emerge in November (Kovalev 1965).

\section{Belizinella vicina Kovalev, 1965}

Synonyms. Belizinella vicina Kovalev, 1965. Trigonaspis vicina: Melika \& Abrahamson (2002); Belizinella vicina Kovalev, 1965, comb rev. in Melika (2012).

Lifecycle. Only the asexual generation is known; females are wingless (Kovalev 1965, Melika 2012).

Geographic distribution. Russian Far East (Kovalev 1965).

Host plants. Quercus mongolica (Kovalev 1965).

Gall location and structure. Leaf galls, very similar to those induced by B. gibbera, but are smaller when mature (Kovalev 1965).

Phenology. Galls mature in August; adults emerge in February of the following year (Kovalev 1965).

\section{Biorhiza Westwood, 1840}

Biorhiza nawai (Ashmead, 1904)

Synonyms: Biorhiza weldi Yasumatsu \& Matsuda, 1955 (in Pujade-Villar et al. 2003).

Lifecycle. Only the sexual generation is known (Monzen 1954).

Geographic distribution. Japan (Ashmead 1904, Monzen 1954), South Korea (Abe et al. 2007); Russia (Far East) (Kovalev 1965), China (Beijing Province) (Abe et al. 2007).

Host plants. Quercus mongolica, Q. serrata (Monzen 1954, Kovalev 1965).

Gall location and structure. Bud gall on the top of a twig The gall is large, globose, about $2 \mathrm{~cm}$ in diameter, succulent, polythalamus, inseparable, green in colour, with reddish tinge on sunny side, turns yellowish in summer (Fig. A140) (Monzen 1954).

Phenology. Adults emerge in June-July (Monzen 1954, Kovalev 1965). 


\section{Callirhytis Förster, 1869}

Callirhytis glanduliferae Monzen, 1953

Lifecycle. Only females are known (Monzen 1953).

Geographic distribution. Japan (Monzen 1953).

Host plants. Quercus serrata (Monzen 1953).

Gall location and structure. The gall is hemispherical, on the underside of leaves, the free surface convex, attached part flat, diameter about $4 \mathrm{~mm}$, yellowish when fresh, turns to yellowish or reddish brown when mature, smooth, hard, monothalamus, the larval chamber in the center of the gall (Fig. A141) (Monzen 1953).

Phenology. Galls mature in October; adults emerge next year in May (Monzen 1953).

\section{Cerroneuroterus Melika \& Pujade-Villar, 2010}

Cerroneuroterus folimargo (Monzen, 1954)

Synonyms. Neuroterus folimargo Monzen, 1954. Cerroneuroterus folimargo: Melika et al. (2010).

Lifecycle. Alternating sexual and asexual generations are known. The asexual generation was described by Monzen (1954); the sexual generation was experimentally obtained by Yukawa \& Masuda (1996).

Geographic distribution. Japan and Taiwan (Melika et al. 2010).

Host plants. Quercus acutissima Carruth., Q. variabilis Blume (Melika et al. 2010).

Gall location and structure. The asexual spangle gall is located on the end of a vein or spine of the leaf, on the underside, pale pinkish, conical, $2 \mathrm{~mm}$ in diameter (Fig. A142). Sexual generation galls are tiny catkin galls (Yukawa \& Masuda 1996).

Phenology. Asexual females emerge in April and induce tiny catkin galls; sexual generation adults emerge in late May (Melika et al. 2010).

Similar galls. The asexual spangle gall very similar to the gall of $C$. vonkuenburgi (Dettmer), but in $C$. folimargo, the gall is located always at the leaf edge, at the end of veins with only a single gall per leaf (Fig. A142) (Melika et al. 2010). The sexual generation galls are very similar to those of $C$. monzeni (Dettmer) (Melika et al. 2010).

\section{Cerroneuroterus monzeni (Dettmer, 1934)}

Synonyms: Neuroterus monzeni Dettmer, 1934. Cerroneuroterus monzeni: Melika et al. (2010). Lifecycle. Alternating sexual and asexual generations are known. The sexual females were originally described by Dettmer (1934). Later, Monzen redescribed the sexual generation including males (Monzen 1954). Yukawa \& Masuda (1996) experimentally matched the sexual and the asexual generations.

Geographic distribution. Japan (Dettmer 1934, Monzen 1954).

Host plants. Quercus acutissima and Q. variabilis (Dettmer 1934, Monzen 1954).

Gall location and structure. Sexual generation galls on catkins; small, conical, yellowish, smooth, with few setae, thin walled, unilocular, $2 \mathrm{~mm}$ in diameter. Small asexual spangle galls develop on the underside of leaves (Fig. A67).

Phenology. The asexual females overwinter in the galls, adults emerge in March-April and induce catkin galls from which the adults emerge in May of the same year (Monzen 1954, Melika et al. 2010). 
Similar galls. Similar in shape to those induced by the Western Palearctic species $N$. numismalis, rounded and flattened with a central dimple, dark red point, without fringe of hair around the gall. Also similar to C. folimargo.

Cerroneuroterus vonkuenburgi (Dettmer, 1934),

Synonyms. Neuroterus vonkuenburgi var. wakayamensis Monzen (in Abe et al. (2007)). Cerroneuroterus vonkuenburgi: Melika et al. (2010); Andricus asakawae Sninji, 1943 (in Yukawa \& Masuda 1996); Andricus kanagawae Shinji, 1943 (in Yukawa \& Masuda 1996); Neuroterus bonihenrici Dettmer, 1934 (in Yukawa \& Masuda 1996); Neuroterus (=Neoneuroterus) kashiyamai (Monzen, 1954) (in Yukawa \& Masuda 1996); Dryophanta mitsukurui Ashmead, 1904 (in Yukawa \& Masuda 1996).

Lifecycle. Alternating sexual and asexual generations are known. The sexual generation was experimentally obtained by Yukawa \& Masuda (1996).

Geographic distribution. Japan (Ashmead 1904, Dettmer 1934, Monzen 1954, Yukawa \& Masuda 1996) and Taiwan (Melika et al. 2010).

Host plants. Quercus acutissima and Q. variabilis (Melika et al. 2010).

Gall location and structure. The asexual galls are on the underside of leaves, yellowish or reddish brown, subspherical, thinner toward the apex and somewhat dilated at the top, densely pubescent, diameter 4-6mm, gall wall thick, monothalamus, larval chamber $1.5 \mathrm{~mm}$ in diameter, separable (Monzen 1954; Fig. A66). Sexual galls form large woolly masses on catkins (Melika et al. 2010). The flower gall, a sponge like ball, diameter 15-25 mm (Fig. A143). Each larval chamber small, oblong, yellowish, thin-walled, covering with woolly hairs, forming a bunch at an edge especially. The chamber 3x2 mm, the hairs 5-6 mm (Monzen 1954).

Phenology. The sexual wasps emerge in late May to early June (Monzen 1954). Asexual spangle galls mature in early November; adults emerge from late November; females were observed to lay eggs from late January (Melika et al. 2010).

\section{Cyclocynips Melika, Tang, \&Sinclair, 2013}

\section{Cyclocynips uberis Melika \&Tang, 2013}

Lifecycle. Only the asexual generation is known (Melika et al. 2013).

Geographic distribution. Taiwan (Fuhsing Township, Taoyuan County) (Melika et al. 2013).

Host plants. Quercus longinux Hayata (Quercus subgenus Cyclobalanopsis) which is endemic to Taiwan, thus raising the possibility that Cyclocynips uberis may also be endemic (Melika et al. 2013).

Gall location and structure. Multi-chambered twig swellings up to $10-15 \mathrm{~cm}$ long and twice the diameter of unaffected parts of the twig (Fig. A144). Larval chambers are 1.3-2.0 mm long and $0.2-0.5 \mathrm{~mm}$ in diameter, located under the bark at various depths throughout the twig. The orientation of the longitudinal axis of the larval chamber varies but is usually parallel to the shoot axis (Fig. A145). The bark of the affected shoot is unaffected, and other than the larval chambers there are no obviously differentiated complex gall tissues within the shoot (Melika et al. 2013).

Phenology. Adults emerge in March (Melika et al. 2013).

Similar galls. These galls broadly resemble those induced by the asexual generation of Plagiotrochus masudai and P. glaucus (Melika et al. 2013). 
Cyclocynips tumorvirgae Melika \&Tang, 2013

Lifecycle. Only the asexual generation is known (Melika et al. 2013).

Geographic distribution. Taiwan (Shihding District, New Taipei City) (Melika et al. 2013).

Host plants. Quercus glauca Thunb. in Murray (Quercus subgenus Cyclobalanopsis) (Melika et al. 2013).

Gall location and structure. Galls develop as multichambered swellings within twigs. Larval chambers are 2.1-2.6 $\mathrm{mm}$ long and 1.2-1.6 $\mathrm{mm}$ in diameter, always appear to be close to the twig surface and orientated along its axis, unlike those of $C$. uberis that can occur at varying depths and orientations (Fig. A146) (Melika et al. 2013).

Phenology. Adults emerge in March (Melika et al. 2013).

Similar galls. Galls are similar to those induced by $C$. uberis.

\section{Cycloneuroterus Melika \& Tang, 2011}

Cycloneuroterus abei Melika \&Tang, 2016

Lifecycle. Only the sexual generation is known (Tang et al. 2016a).

Geographic distribution. Taiwan (Shihding District, New Taipei City; Taoyuan County; Heping District, Taichung City) (Tang et al. 2016a).

Host plants. Quercus glauca and Q. globosa (Tang et al. 2016a).

Gall location and structure. The gall is an integrated young leaf swelling, protruding on both sides of the leaf blade (on $Q$. glauca galls were found to grow on the leaf petiole). The gall is 2.5-6.0 $\mathrm{mm}$ in diameter, with a single or multiple larval chambers. Sometimes the gall growth causes distortion of leaves (Figs A147-148) (Tang et al. 2016a).

Phenology. Gall growth coincides with the point of host sprouting in mid-February. Galls develop to largest size when the host leaves wholly expanded; adults emerge in late March till mid-April (Tang et al. 2016a).

Cycloneuroterus akagashiphilus Ide, Wachi \& Abe, 2012

Lifecycle. Only the sexual generation is known (Ide et al. 2012).

Geographic distribution. Japan (Kyushu) (Ide et al. 2012).

Host plants. Quercus acuta Thunb. (Quercus subgenus Cyclobalanopsis) (Ide et al. 2012).

Gall location and structure. Galls on lateral margin or apex of young leaf just after bud burst in late May. Oval gall $2.0 \mathrm{~mm}$ in diameter, , usually two to eight galls clustered. Immature galls yellowish green, mature ones are brown (Ide et al. 2012).

Phenology. Gall mature in late March-April; adults emerge from late May to early June (Ide $e t$ al. 2012).

Cycloneuroterus arakashiphagus Ide,Wachi \& Abe, 2012

Lifecycle. Alternating sexual and asexual generations are known (Yukawa \& Masuda 1996, Ide et al. 2012).

Geographic distribution. Japan (Honshu, Shikoku, Kyushu) (Yukawa \& Masuda 1996, Ide et al. 2012).

Host plants. Quercus glauca (Ide et al. 2012).

Gall location and structure. The sexual generation galls develop in sprouts, while the galls of the asexual generation - on leaf veins. The sexual generation galls are oval, pale green, or 
yellowish brown, 1.5-2.0 $\mathrm{mm}$ in diameter and 2.0-2.5 $\mathrm{mm}$ in height, usually clustered (Ide et al. 2012).

Phenology. The adult of the asexual generation is unknown. Galls of the sexual generation appear in early April; adults of the sexual generation emerge in mid-April. The gall of the asexual generation begins to appear on the leaf veins in early June; after maturation, the asexual generation gall falls to the ground (Ide et al. 2012).

\section{Cycloneuroterus ergei Tang \& Melika, 2016}

Lifecycle. Only the sexual generation is known (Tang et al. 2016a).

Geographic distribution. Taiwan (Mt. Erge, Shihding District, New Taipei City) (Tang et al. 2016a).

Host plants. Quercus salicina Blume (Quercus subgenus Cyclobalanopsis) (Tang et al. 2016a). Gall location and structure. Galls are on young sprouts, unilocular, pinkish or yellowish, 2.0$2.5 \mathrm{~mm}$ in length (Figs A149) (Tang et al. 2016a).

Phenology. Galls mature in late March; adults emerge from galls immediately after field collection (Tang et al. 2016a).

Cycloneuroterus formosanus Tang \& Melika, 2011

Lifecycle. Only the sexual generation is known (Tang et al. 2011a).

Geographic distribution. Taiwan (Taichung, Nantou, and Taitung Counties).

Host plants. Lithocarpus konishii (Hayata) Hayata (Tang et al. 2011a).

Gall location and structure. The gall is an irregular stem-swelling that only appears on young spring growth. The gall causes a swelling, occuring both along shoots or in terminal buds, and young leaves remain on both swollen shoots or swollen terminal buds (Figs A150-151). Each gall is multilocular and coloured red with a slight purple tinge. The gall can grow to a length of $25 \mathrm{~mm}$. The young gall is fleshy and juicy, remaining soft until adults emerge (Tang et al. 2011a).

Phenology. Mature galls were collected from early to mid April; adults emerge immediately after gall collection under laboratory conditions (Tang et al. 2011a).

Comments. Formally only the sexual generation is known (Tang et al. 2011a). However, sequences of cytb and ITS2 genes showed that this species has an alternate asexual generation which is developing also on L. konishii and produces detachable leaf galls (Fig. A152). No adult wasps were obtained yet from these galls and thus the asexual generation formally is not described yet.

\section{Cycloneuroterus fortuitusus Tang \& Melika, 2011}

Lifecycle. Only the sexual generation is known (Tang et al. 2011a).

Geographic distribution. Taiwan (Taitung County) (Tang et al. 2011a) and Japan (Kitayama, Kofu City, Yamanashi Prefecture and Mt. Sakurei, Saga Prefecture) (Ide et al. 2012). It is possible that this species is also distributed on the Asian continent where Q. glauca occurs (Himalaya to Japan and Vietnam (Govaerts \& Frodin 1998)).

Host plants. Quercus glauca (Tang et al. 2011a).

Gall location and structure. Integral swelling-like galls in the leaf petiole (Fig. A153). The gall is cryptic, occuring at the base of a newly grown shoot that is still covered by many bud scales. The gall causes a slight swelling at the base of the young shoot; unless the bud scales are removed, it is difficult to detect the presence of the gall (Tang et al. 2011). The gall is $3 \mathrm{~mm}$ in 
height and $1.5 \mathrm{~mm}$ in diameter. Sometimes two galls occur together at the base of the same young shoot (Tang et al. 2011a).

Phenology. Galls mature in late February; adults emerge in early March (Tang et al. 2011a).

\section{Cycloneuroterus gilvus Melika \& Tang, 2016}

Lifecycle. Only the sexual generation is known (Tang et al. 2016a).

Geographic distribution. Taiwan (Hsinchu and Taoyuan Counties) (Tang et al. 2016a).

Host plants. Quercus gilva Blume (Quercus subgenus Cyclobalanopsis) (Tang et al. 2016a).

Gall location and structure. The gall is hollow or blister-like on folded or unfolded young leaves. Galls with a single larval chamber, usually numerous galls develop on one leaf, pinkish or rusty in color (Figs A154-155). Galls are 3.0-4.7 mm in diameter and 2.2-4.8 mm in height (Tang et al. 2016a).

Phenology. The gall development coincides with host sprouting in mid-March ; adults emerge from late March to early April (Tang et al. 2016a).

\section{Cycloneuroterus globosus Melika \& Tang, 2016}

Lifecycle. Only the sexual generation is known (Tang et al. 2016a).

Geographic distribution. Taiwan (Nantou County). The host-plant, Q. globosa is endemic to Taiwan, thus $C$. globosus might be also endemic to Taiwan (Tang et al. 2016a).

Host plants. Quercus globosa (Tang et al. 2016a).

Gall location and strucrure. Galls are red pimples on midribs or lateral veins of young leaves. In some cases the gall development disrupts the sprouting and galls are forming a tuft on a bud (Figs A156-158). Galls are unilocular, $1.0-1.7 \mathrm{~mm}$ in width and $1.6-3.3 \mathrm{~mm}$ in length (Tang et al. 2016a).

Phenology. The gall development coincides with sprouting from mid- to late-March; adults emerge under laboratory conditions immediately after the galls were collected in the field (Tang et al. 2016a).

Cycloneuroterus hisashii Ide, Wachi \& Abe, 2012

Lifecycle. Only the sexual generation is known (Ide et al. 2012).

Geographic distribution. Japan (Honshu) (Yukawa \& Masuda 1996, Ide et al. 2012).

Host plants. Quercus glauca (Ide et al. 2012).

Gall location and structure. The gall is in the basal portion of a bud, globular, 5-7 mm in diameter and $10-13 \mathrm{~mm}$ in height. Galled bud somewhat swollen, with 15 or less larval chambers (Ide et al. 2012).

Phenology. Galls mature from mid- to late April; adults emerge in early May (Ide et al. 2012).

\section{Cycloneuroterus jianwui Tang \& Melika, 2016}

Lifecycle. Only the sexual generation is known (Tang et al. 2016a).

Geographic distribution. China (Yunnan Province, Lan Cang Co., Mt. Xinghou) (Tang et al. 2016a).

Host plants. The host plant species is unknown but it was definitely a species from Quercus subgenus Cyclobalanopsis (Tang et al. 2016a).

Gall location and structure. The gall growth causes young leaves highly distorted; those leaves usually occur on the tip of the twigs (Fig. A159). Galls are multilocular, 2.0-2.5 $\mathrm{mm}$ in diameter and 1.5-2.0 $\mathrm{mm}$ in height (Tang et al. 2016a). 
Phenology. Galls appear in the period of active sprouting; adults emerge in April (Tang et al. 2016a).

Cycloneuroterus lilungi Tang, Melika \& Yang, 2011

Lifecycle. Only the sexual generation is known (Tang et al. 2011a).

Geographic distribution. Taiwan (Pingtung and Hsinchu Counties). The host plant, $Q$. longinux, is known only from Taiwan (Govaerts \& Frodin 1998), so it is possible that this species is endemic to Taiwan (Tang et al. 2011).

Host plants. Quercus longinux (Tang et al. 2011a).

Gall location and structure. Integral leaf galls. The young galls are red, succulent pimples on young leaves with central larval chamber. Mature galls are brownish and hollow, without conspicuous central larval chamber (Figs A160). Diameter of galls is 0.8-1.6 mm. The tissues surrounding larval chamber are swollen and cause deformation of leaves (Tang et al. 2011a).

Phenology. Galls mature in early and mid-February; adults emerge from late February till early March (Tang et al. 2011a).

\section{Cycloneuroterus lirongchiuea Melika \& Tang, 2011}

Lifecycle. Only the sexual generation is known (Tang et al. 2011a).

Geographic distribution. Taiwan (Taitung County). Quercus hypophaea Hayata is known only from Taiwan (Govaerts \& Frodin 1998), so it is possible that this species is endemic to Taiwan (Tang et al. 2011a).

Host oaks: Q. hypophaea (Quercus subgenus Cyclobalanopsis) (Tang et al. 2011a).

Gall location and structure. Galls are integral to leaf blades of young leaves with swelling on both sides of the leaf. They are brownish, 3-4 $\mathrm{mm}$ in diameter and extending up to $2.7 \mathrm{~mm}$ from the leaf surface. Galls grow individually on a leaf rather than aggregated together (Figs A161162). Gall tissue is succulent when young and the gall becomes hollow once the larva matures and pupates inside. The pupa can roll freely inside the gall (Tang et al. 2011a).

Phenology. Galls mature from early March until early April; adults emerge under laboratory conditions over the same time period (Tang et al. 2011a).

\section{Cycloneuroterus lohsei Melika \& Tang, 2016}

Lifecycle. Alternating sexual and asexual generations are known (Tang et al. 2016a). Geographic distribution. Taiwan (Shihding District, New Taipei City; Hsinchu County) (Tang et al. 2016a).

Host plants. Quercus sessilifolia Blume (Quercus subgenus Cyclobalanopsis) for the sexual generation and $Q$. gilva (Quercus subgenus Cyclobalanopsis) for the asexual generation galls (Tang et al. 2016a).

Gall location and structure. Galls of the sexual generation are multilocular, fully covered with pink or white hairs, and on young leaves. The gall growth sometimes causes the deformation of the whole leaf. The swollen parts on leaves are 10.0-20.0 mm long and 4.0-6.0 mm broad (Figs A163-164). Asexual generation galls are multilocular, rounded clusters on the midrib on the lower surface of mature leaves and densely covered in brown hairs, 4-5 mm long (Fig. A165) (Tang et al. 2016a).

Phenology. Sexual generation galls appear on the host-plant in March; adults emerge from lateMarch to mid-April. Asexual generation galls have not been reared successfully, so asexual adults are unknown. However, DNA sequences obtained from larvae dissected from asexual 
galls match those from sexual generation adults. Asexual generation galls have been collected in August (Tang et al. 2016a).

Cycloneuroterus longinuxus Tang \& Melika, 2011

Lifecycle. Only the sexual generation is known (Tang et al. 2011a).

Geographic distribution. Taiwan (New Taipei City, Hsinchu, Nantou, and Taitung Counties).

Host plants. Quercus longinux (Tang et al. 2011a).

Gall location and structure. Integral leaf galls. The gall is a multilocular cluster of many small spherical swellings extending to both sides of the leaf blade. The gall tissue is succulent and soft until the adults emerge and coloured the same as the leaf (Fig. A166). Viewed from the upper side, the swollen section of leaf is $6-10 \mathrm{~mm}$ in width and $12 \mathrm{~mm}$ long. After the emergence of adults the gall turns liginified and brownish (Tang et al. 2011a).

Phenology. Galls mature in early March; adults emerge under laboratory conditions immediately after collecting (Tang et al. 2011a).

\section{Cycloneuroterus tumiclavus Tang \& Melika, 2016}

Lifecycle. Only the sexual generation of this species is known (Tang et al. 2016a).

Geographic distribution. Taiwan (Heping District) (Tang et al. 2016a).

Host plants. Quercus glauca (Tang et al. 2016a).

Gall location and structure. The galls are on the adaxial end of the catkin spike, yellow green, oval-shaped swellings densely covered with hairs, $3.0-3.5 \mathrm{~mm}$ in length and $1.5-2.0$ in width (Fig. A167). Galls are unilocular (Tang et al. 2016a).

Phenology. Galls mature in late March; adults emerge under laboratory conditions immediately after the galls were transferred to the laboratory for rearing (Tang et al. 2016a).

\section{Cycloneuroterus uraianus Tang \& Melika, 2016}

Lifecycle. Only the sexual generation is known (Tang et al. 2016).

Geographic distribution. Taiwan (Taoyuan County, Fushing Township) (Tang et al. 2016a).

Host plants. Castanopsis uraiana (Hayata) Kaneh. \& Hatus (Tang et al. 2016a).

Gall location and structure. Bud galls. Galls are cryptic and embedded in buds with 2-15 cells clustered together. Infected buds remain their intact appearance and it is difficult to detect whether the bud was infected or not (Fig. A168) (Tang et al. 2016a).

Phenology. Galls mature in late March; adults emerge from late March till early April (Tang et al. 2016a).

Cycloneuroterus wangi Abe, Ide, \& Odagiri, 2014

Lifecycle. Only the asexual generation is known (Abe et al. 2014a).

Geographic distribution. Southeastern China (Nanling National Forest Park, Ruyuan, Shaoguan, Guangdong) (Abe et al. 2014a).

Host plants. Q. sessilifolia (Abe et al. 2014a).

Gall location and structure. Galls unknown.

Phenology. Adults were collected in December (Abe et al. 2014a).

\section{Cynips Linnaeus, 1758}

Cynips staminobia Kovalev, 1965

Lifecycle. Only the sexual generation is known (Kovalev 1965). 
Geographic distribution. Far East Russia (Kovalev 1965).

Host plants. Quercus mongolica (Kovalev 1965).

Gall location and structure. Rounded unilocular catkin galls with white hairs, $3 \mathrm{~mm}$ in diameter (Kovalev 1965).

Phenology. Adults emerge in early June (Kovalev 1965).

\section{Dryocosmus Giraud, 1859}

\section{Dryocosmus carlesiae Tang \& Melika, 2011}

Lifecycle. Only the sexual generation is known (Melika et al. 2011).

Geographic distribution. Taiwan (Taitung and Pingtung Counties) (Melika et al. 2011).

Host plants. Castanopsis carlesii (Hemsley) Hayata (Melika et al. 2011).

Gall location and structure. Unilocular galls develop from leaf buds or on leaves. The body of the gall located on a long, thin stalk, $10-20 \mathrm{~mm}$ long, growing out from the bud or forming within the leaf petiole. The body of the gall rounded, spherical, $7.0-8.3 \mathrm{~mm}$ in diameter. The gall is green with the surface smooth, shiny, mottled with some whitish irregular spots (Figs A169170). The parenchyma of the gall is green, juicy, with a centrally located larval chamber, 3.0-3.5 $\mathrm{mm}$ in length and diameter usually half of the length (Melika et al. 2011).

Phenology. Galls appear on the tree from early March, mature by mid- to late March; adults emerge in April (Melika et al. 2011).

\section{Dryocosmus kunugiphagus Ide \& Abe, 2015}

Lifecycle. Alternate sexual and asexual generations are known (Ide \& Abe 2015).

Geographic distribution. Japan (Honshu, Shikoku, Kyushu) and Korea (Yukawa \& Masuda 1996, Ide \& Abe 2015).

Host plants. Quercus acutissima (Ide \& Abe 2015).

Gall location and structure. Asexual generation galls usually are clustered on twig, each gall is unilocular, spherical, 5-6 mm in diameter. Sexual generation unilocular spherical galls are on shoot apex.

Phenology. Sexual generation galls are induced when shoots of the host plant elongate and galls reach maturation in late May; adults emerge in early June. Adult females oviposit into the previous year's shoot. Asexual generation galls begin appearing in early August; adults emerge between late March and early April (Ide \& Abe 2015).

\section{Dryocosmus kuriphilus Yasumatsu, 1951}

Lifecycle. Only the asexual generation is known (Murakami 1980).

Geographic distribution. Dryocosmus kuriphilus was formally described in 1951 (Yasumatsu 1951), but was first observed in China in 1929 (Murakami 1980). Murakami (1980) concluded that this gallwasp is indigenous to China and was accidentally introduced to Japan in $c .1941$ (Yasumatsu 1951), Korea in 1958 (Tamura 1962, Cho \& Lee 1963), the USA in 1974 (Payne et al. 1975, 1976), Europe (Piedmont, Italy) in 2002 (Brussino et al. 2002), Nepal (Ueno 2006) and Taiwan in 2010 (Tang 2015). The history of its introduction worldwide and particularly in Europe is described in depth by Aebi et al. (2006). Currently D. kuriphilus spreaded throughout Europe, where Castanea spp. is growing (Kos et al. 2015, Radócz et al. 2015).

Host plants. Originally in China D. kuriphilus trophically associated with Castanea crenata Siebold in Zucc., C. henryi (Skan), C. mollissima Blume, C. seguinii Dode. Later on, after 
accidental introduction to the USA and Europe, the pest shifted also onto $C$. dentata (Marshall) Borkh. and C. sativa Mill. respectively (Brussino et al. 2002).

Gall location and structure. Subglobular, succulent, and fleshy integral multilocular galls on young buds and leaf petiole or leaf midrib. Galls are usually green, about $5.0 \mathrm{~mm}$ in width by 5.0-15.0 mm in length (Fig. A171). The average number of larval chambers per gall is 1.5-3.0 (Brussino et al. 2002).

Phenology. Asexual female D. kuriphilus emerges in early summer and lays eggs in buds of chestnut trees; the first-instar larvae are overwintering in the buds, and galls start to develop in spring at bud burst; adults emerge in June-August (Brussino et al. 2002).

Dryocosmus nanlingensis Abe, Ide, \& Odagiri, 2014

Lifecycle. Only the sexual generation is known (Abe et al. 2014a).

Geographic distribution. Southeastern China (Nanling National Forest Park, Ruyuan, Shaoguan, Guangdong) (Abe et al. 2014a).

Host plants. Unknown.

Gall location and structure. Unknown.

Phenology. Adults emerge in December (Abe et al. 2014a).

Dryocosmus okajimai Abe, Ide, Konishi \& Ueno, 2014

Lifecycle. Only the sexual generation is known (Abe et al. 2014b).

Geographic distribution. Vietnam (Tuyen Lam Lake, Da Lat, Lam Dong Province) (Abe et al. 2014b).

Host plants. Unknown.

Gall location and structure. Unknown.

Phenology. Adults emerge in July (Abe et al. 2014b).

Dryocosmus pentagonalis Melika \& Tang, 2011

Lifecycle. Only the sexual generation is known (Melika et al. 2011).

Geographic distribution. Taiwan (Taitung County) (Melika et al. 2011).

Host plants. Castanopsis carlesii (Melika et al. 2011).

Gall location and structure. Galls are forming on buds, catkins and young leaves. On catkins this gall causes thickening of the petiole, with the base of the gall inserted in a shallow depression. An elongated, spindle-shaped unilocular gall reaching a length of 8-10 mm and a diameter of 2-4 mm when mature. The gall is attached by a stalk of variable length (commonly 4-12 mm) (Figs A172-173). The main body of the gall is marked with longitudinal ribs, usually five, and thus from the top view the gall is star-shaped (Melika et al. 2011).

Phenology. Galls appear on the tree from early March, mature by mid-March; adults emerge in late March to the first week of April (Melika et al. 2011).

Similar galls. The gall closely resembles the asexual galls of three Western Palearctic Andricus species: A. callidoma (Hartig), A. malpighii (Adler) and A. seminationis (Giraud); structurally the last one is the most similar (Melika et al. 2011).

Dryocosmus sakureiensis Ide, Wachi \& Abe, 2013

Lifecycle. Only the sexual generation is known (Ide et al. 2013).

Geographic distribution. Japan (Kyushu) (Ide et al. 2013).

Host plants. Quercus sp. (Quercus subgenus Cyclobalanopsis) (Ide et al. 2013). 
Gall location and structure. One to three galls in one bud. Oval, $2.5-4.0 \mathrm{~mm}$ in height, $1.5-2.0$ $\mathrm{mm}$ in diameter. Young galls yellowish green, mature ones brown. Apex of galled bud sometimes more or less bent (Ide et al. 2013).

Phenology. Galls mature from late April to early May; adults emerge from mid- to late May (Ide et al. 2013).

Dryocosmus sefuriensis Ide, Wachi \& Abe, 2013

Lifecycle. Only the sexual generation is known (Ide et al. 2013).

Geographic distribution. Japan (Kyushu) (Ide et al. 2013).

Host plants. Quercus acuta Thunb. (Quercus subgenus Cyclobalanopsis) (Ide et al. 2013).

Gall location and structure. One to three galls on one young sprout. Gall irregularly swollen, pubescent; sprout with gall shortened and swollen. Young galls yellowish green and turn brown when mature; one gall contains 3-6 larval chambers, each larval chamber $1.5-2.0 \mathrm{~mm}$ in diameter (Ide et al. 2013).

Phenology. Galls mature in late May; adults emerge in late May to early June (Ide et al. 2013).

Dryocosmus squamus (Monzen, 1953), comb. nova

Synonyms. Andricus squama Monzen, 1953. Dryocosmus squamus (Monzen, 1953), comb. nova.

Lifecycle. Only the sexual generation is known (Monzen 1953).

Geographic distribution. Japan (Iwate Prefecture) (Monzen 1953).

Host plants. Quercus serrata (Monzen 1953).

Gall location and structure. The bud gall is small, oblong, diameter about 4-6 mm, height 4.5 $\mathrm{mm}$, the surface is covered with microscopical scales, yellowish brown; the gall wall rather thick, with 1-3 larval chambers (Monzen 1953, Yukawa \& Masuda 1996).

Phenology. Adults emerge in late May (Monzen 1953).

Comments. Three females were found in the Monzen collection at the Kyoto University by G.Melika. They are all Dryocosmus and not Andricus (the mesoscutum is smooth, without surface sculpture, scuto-scutellar articulation present, the mesoscutellum without scutellar foveae, only a semilunar depression anteriorly present on the mesoscutellum).

Dryocosmus testisimilis Tang \& Melika, 2011

Lifecycle. Only the sexual generation is known from galls (Melika et al. 2011).

Geographic distribution. Taiwan (New Taipei City, Taoyuan and Hsinchu Counties) (Melika et al. 2011).

Host plants. Castanopsis uraiana (Melika et al. 2011).

Gall location and structure. Galls on leaves, 22-31 mm in length and 12-21 mm in diameter. The parenchyma of the gall is green, juicy, with many larval chambers gather centrally. Each larval chamber is $2.5-3.0 \mathrm{~mm}$ in length and $1.7-2.0 \mathrm{~mm}$ in diameter. Multilocular. The length of the gall body usually encompasses around $2 / 3$ of the entire length of the gall, including the length of the stalk (Fig. A174) (Melika et al. 2011).

Phenology. Galls mature in mid- and late-March; adults emerge in April-May (Melika et al. 2011).

Dryocosmus triangularis Melika \& Tang, 2011

Lifecycle. Only the sexual generation is known (Melika et al. 2011). 
Geographic distribution. Taiwan (Taitung County) (Melika et al. 2011).

Host plants. Castanopsis carlesii (Melika et al. 2011).

Gall location and structure. Galls are forming on buds, catkins, and young leaves (Melika et al. 2011). The gall has only three distinct longitudinal ribs, thus has a triangular cross-section; 4.5$7.0 \mathrm{~mm}$ long, $1.2-1.9 \mathrm{~mm}$ width in crosssection; the stalk is usually much shorter than in $D$. pentagonalis, only $2-3 \mathrm{~mm}$ long (Fig. A175) (Melika et al. 2011).

Phenology. Galls appear on the tree from early March, mature in mid-March; adults emerge in late March-April (Melika et al. 2011).

\section{Dryocosmus zhuili Liu \& Zhu, 2015}

Lifecycle. Only the sexual generation is known (Zhu et al. 2015).

Geographic distribution. Southeastern China (Fujian province) (Zhu et al. 2015).

Host plants. Castanea henryi (Zhu et al. 2015).

Gall location and structure. Subglobular, succulent, and fleshy integral galls are formed on young buds and leaf petiole and midrib of leaves close to leaf base on new shoots. Galls are usually green, occasionally rosy on the sunny side; about $8.0 \mathrm{~mm}$ in width by $8.0-25.0 \mathrm{~mm}$ in length (Figs 4-6 in Zhu et al. 2015).

Phenology. The same as in D. kuriphilus.

Similar galls. It is impossible to distinguish galls and adults from D. kuriphilus.

Twelve Dryocosmus species from China and Taiwan were recently described (Tang et al. 2016c, accepted)

\section{Latuspina Monzen, 1954}

Latuspina abemakiphila Ide \& Abe, 2016

Lifecycle. Only the sexual generation is known (Ide \& Abe 2016).

Geographic distribution. Japan (Honshu, Shikoku) (Ide \& Abe 2016, Yukawa \& Masuda 1996).

Host plants. Quercus variabilis (Ide \& Abe 2016).

Gall location and structure. Several to a dozen galls are clustered on leaf blade; pale green, blister-like, $1.5-2.5 \mathrm{~mm}$ in diameter, $1.5 \mathrm{~mm}$ in height on upper side, white, circular disk-like, $4.0-5.0 \mathrm{~mm}$ in diameter, $1.0-2.0 \mathrm{~mm}$ in height on lower side, containing one larval chamber (Ide \& Abe 2016).

Phenology. Galls appear in mid-April; adults emerge from late April to early May (Ide \& Abe 2016).

\section{Latuspina acutissimae Wang, Pujade-Villar \& Guo, 2016}

Lifecycle. Only the sexual generation is known (Wang et al. 2016b).

Geographic distribution. Currently known from China: Zhejiang (Wang et al. 2016b).

Host plants. Q. acutissima (Wang et al. 2016).

Gall location and structure. Unilocular integrated pabrenchima leaf galls. The young growing gall is juicy, spherical, green with black spots, with small surface tubercles, compressed if closely clustered. The mature gall is brown, up to $1.2 \mathrm{~mm}$ in diameter with hard walls (Wang et al. 2016b). 
Phenology. Galls are appearing on the tree from early May, maturing through the summer and in late October. Under the laboratory conditions, adults emerge from late September (Wang et al. 2016).

\section{Latuspina atamiensis (Ashmead, 1904)}

Synonyms: Neuroterus atamiensis Ashmead, 1904; Latuspina atamiensis: Ide \& Abe (2016).

Lifecycle. Alternating sexual and asexual generations are known (Ide \& Abe 2016).

Geographic distribution. Japan (Honshu, Kyushu) (Ide \& Abe 2016).

Host plants. Quercus acutissima, Q. variabilis (Ide \& Abe 2016).

Gall location and structure. Sexual unicolular (sometimes several galls) are on young acorn cup. Galls are pale green or brownish green, oval, pointed at apex, with one larval chamber, 2.0 $\mathrm{mm}$ in diameter, $4.0 \mathrm{~mm}$ in height, covered with dense, short, white pubescence. Asexual galls, usually one to three galls, are on a leaf petiole; galls are pinkish red or blackish purple, oval, containing one larval chamber, $1.0-1.5 \mathrm{~mm}$ in diameter, $1.5-2.0 \mathrm{~mm}$ in height (Yukawa \& Masuda 1996, Ide \& Abe 2016).

Phenology. The life cycle was described by Yukawa and Masuda (1996). The sexual generation galls appear in late April and mature in mid-May; adults emerge in mid- or late June and females lay eggs in the petioles of young leaves. The galled petioles begin to thicken in early July. The asexual generation galls appear from mid-July to September and mature in 7-10 days after their appearance. The mature galls fall to the ground; adults emerge from following March and lay eggs in juvenile acorns (Ide \& Abe 2016).

\section{Latuspina hellwegi (Dettmer, 1934)}

Synonyms: Neuroterus hellwegi Dettmer, 1934; Latuspina hellwegi: Ide \& Abe (2016).

Lifecycle. Alternating sexual and asexual generations are known (Ide \& Abe 2016).

Geographic distribution. Japan (Honshu, Shikoku, Kyushu) (Yukawa \& Masuda 1996).

Host plants. Quercus acutissima for both generations (Ide \& Abe 2016).

Gall location and structure. The sexual generation galls are at the base of male flowers; yellowish green or brown, spindleshaped, pointed at apex, containing one larval chamber, 1.5$2.0 \mathrm{~mm}$ in diameter, $3.0-4.0 \mathrm{~mm}$ in height, polished, smooth with pubescence at apex. Asexual generation unilocular galls are on the upper and lower sides of leaf vein, mainly midrib; hornshaped, pointed at the apex, containing one larval chamber, $1.5 \mathrm{~mm}$ in diameter, $2.5-4.0 \mathrm{~mm}$ in height, with several vertical shallow striae on the surface. Immature galls green, mature ones yellowish green.

Phenology. The life cycle was described by Yukawa \& Masuda (1996). The sexual generation galls begin to grow just before the buds burst and mature in mid-April; adults emerge from midto late April. The asexual generation galls begin to appear from mid-May and mature in 7-10 days after their appearance. After maturation, they fall to the ground from late May to late June. The asexual generation adults emerge in September of the same year (ide \& Abe 2016).

\section{Latuspina kofuensis Ide \& Abe, 2016}

Lifecycle. Alternating sexual and asexual generations are known (Ide \& Abe 2016).

Geographic distribution. Japan (Honshu, Shikoku, Kyushu), the Korean Peninsula (Yukawa \& Masuda 1996).

Host plants. Quercus acutissima (Ide \& Abe 2016). 
Gall location and structure. The sexual generation galls are on leaf vein; pale green, oval, containing one larval chamber, $1.0-1.5 \mathrm{~mm}$ in diameter, $1.5-2.0 \mathrm{~mm}$ in height, covered with dense, gray white fine pubescence; the galled leaf distorted. The asexual generation unilocular galls are on midrib of both the upper and lower sides of leaf; yellowish white, pale green, yellowish red, or brown, oval, containing one larval chamber, $1.5-2.0 \mathrm{~mm}$ in diameter, smooth, polished (Ide \& Abe 2016).

Phenology. The life cycle was described by Yukawa \& Masuda (1996). The sexual generation galls appear in late April; adults emerge in mid-May. The asexual generation galls appear from late August to late fall and mature in about 10 days after their appearance. The mature galls fall to the ground; adults from late March to early April of the following year (Ide \& Abe 2016).

\section{Latuspina manmiaoyangae Melika \& Tang, 2012}

Lifecycle. Only the sexual generation is known (Tang et al. 2012a).

Geographic distribution. Taiwan (Taoyuan and Nantou Counties, Taichung City) (Tang et al. 2012a).

Host plants. Quercus variabilis (Tang et al. 2012a).

Gall location and structure. Galls start to develop on young leaves. They are oval, purplish, hairy, $2.5-3.0 \mathrm{~mm}$ long and $1.4-1.8 \mathrm{~mm}$ wide. Galls protrude mainly on the lower leaf surfaces. Galls are single chambered with soft and juicy tissues surrounding the larval chambers of the young that turn hollow when the larvae mature (Figs A176-177) (Tang et al. 2012a).

Phenology. Galls start to grow in mid-February; adults emerge from late February until midMarch (Tang et al. 2012a).

Latuspina nawai (Ashmead, 1904)

Synonyms: Neuroterus nawai Ashmead, 1904; Latuspina nawai: Ide \& Abe (2016).

Lifecycle. Alternating sexual and asexual generations are known (Ide \& Abe 2016).

Geographic distribution. Japan (Honshu, Shikoku, Kyushu) (Yukawa \& Masuda 1996).

Host plants. Quercus acutissima and Q. variabilis for both generations (Ide \& Abe 2016).

Gall location and structure. The sexual generation galls on vein on the lower side of leaf; yellowish white, oval, containing one larval chamber, $2.5-3.0 \mathrm{~mm}$ in diameter, $2.0 \mathrm{~mm}$ in height, covered with dense, white fine pubescence. The asexual generation galls are attached to midrib on lower side of leaf; each gall yellowish brown with brown scattered spots, oval or oblate, containing one larval chamber, $2.5 \mathrm{~mm}$ in diameter, $2.0 \mathrm{~mm}$ in height, matte (Ide \& Abe 2016).

Phenology. The life cycle was described by Yukawa \& Masuda (1996). The sexual generation galls mature in early May and fall to the ground. The sexual generation adults emerge from the galls from early to mid-May. The asexual generation galls appear from late July; mature asexual generation galls fall to the ground from early August to late October; adults emerge the following spring (Ide \& Abe 2016).

\section{Latuspina shaanxinensis Wang, Pujade-Villar \& Guo, 2016}

Lifecycle. Only the sexual generation is known (Wang et al. 2016b).

Geographic distribution. China: Shaanxi, Houzhenzi (Wang et al. 2016b).

Host plants. Quercus sp. (Wang et al. 2016b).

Gall location and structure. An integral, almost discoid leaf gall swelling locates on leaf midrib with irregularly shaped, single larval chamber. Mature galls brown, up to $1.5 \mathrm{~mm}$ in diameter (Wang et al. 2016b). 
Phenology. Galls are appearing on the tree from early May, developing and maturing through the summer and early autumn. Under the laboratory conditions, adults emerge from late September (Wang et al. 2016b).

\section{Latuspina stirps Monzen, 1954}

Synonyms: Neuroterus (Latuspina) stirps Monzen, 1954. Latuspina stirps: Melika et al. (2010). Lifecycle. Alternating sexual and asexual generations are known (Ide \& Abe 2016).

Geographic distribution. Japan (Honshu, Kyushu) (Monzen 1954, Melika et al. 2010, Ide \& Abe 2016), South Korea (Melika et al. 2010, Ide \& Abe 2016).

Host plants. Quercus acutissima for both generations (Monzen 1954, Ide \& Abe 2016).

Gall location and structure. The sexual galls are on the bark; gregarious, small subspherical galls (3-4 mm high and 2.0-2.5 $\mathrm{mm}$ in diameter), protruding from underneath the bark (Fig. A178) (Monzen 1954, Ide \& Abe 2016). The asexual generation galls usually clustered into two to four galls on the midrib on the upper side of leaf; each gall is oval, containing one larval chamber, $1.5-2.0 \mathrm{~mm}$ in diameter, covered with thorns. Young gall is green, mature one pinkishred or red-brown (Ide \& Abe 2016).

Phenology. The sexual generation galls appear in early April and mature in late April; adults emerge from mid- to late May, and the females lay eggs in the midribs of young leaves. The asexual generation galls appear from late July to September; the mature galls begin to fall to the ground in mid-August; the asexual generation adults emerge from December to the following February and lay their eggs in stunted winter buds or latent buds (Ide \& Abe 2016).

Comments. Sequences of three molecular markers (cytb, ITS2, D2 28S region) showed to be identical with those obtained for $N$. hakonensis, thus $L$. stirps might be well the alternate sexual generation of $N$. hakonensis (unpublished data).

\section{Neuroterus Hartig, 1840}

\section{Neuroterus abdominalis Pujade-Villar \& Wang, 2016}

Lifecycle. Only the asexual generation is known (Pujade-Villar et al. 2016).

Geographic distribution. China (Zhejiang) (Pujade-Villar et al. 2016).

Host plants. Quercus acutissima (Pujade-Villar et al. 2016).

Gall location and structure. Ox horn-shaped, unilocular galls on the main or lateral veins of leaf. The galls are $1.5-2.0 \mathrm{~cm}$ long, milk white young galls turning to yellowish green and hollow with a hard and thin woody wall $2.0-2.5 \mathrm{~mm}$ thick when they become mature, and inside the interior space contains a single and free larval chamber (Pujade-Villar et al. 2016).

Phenology. Galls mature in September; adults emerge next year in early March (Pujade-Villar et al. 2016).

\section{Neuroterus gemma Monzen, 1954}

Synonyms. Yukawa \& Masuda (1996) suggested that this species is an inquiline, however, based on the description given by Monzen (1954) it is definitely Neuroterus.

Lifecycle. Only the asexual generation is known (Monzen 1954).

Geographic distribution. Japan (Monzen 1954).

Host plants. Quercus serrata, Q. dentata (Monzen 1954).

Gall location and structure. The leaf gall attached by a short petiole to the underside of leaf. 
Galls are small, subspherical, smooth, yellowish or reddish in color, separable, unilocular, $5 \mathrm{~mm}$ in diameter, larval chamber small, in the innerside above the petiole (Monzen 1954).

Phenology. Galls matue in late autumn; adults emerge next year in April-May (Monzen 1954).

\section{Neuroterus haasi Kieffer, 1904}

Lifecycle. Only the asexual generation is known

Geographic distribution. India (Bengal, Kurseong) (Kieffer 1904, Dalla Torre \& Kieffer 1910, Mani 2000).

Host plants. In current classification, Quercus spicata Smith, which from this cynipid species was described, has been synonymized to Lithocarpus elegans (Blume) (Govaerts \& Frodin 1998).

Gall location and structure. Induces gregarious blister-like galls on very young shoots (Kieffer 1906, Dalla Torre \& Kieffer 1910).

Comments. Originally the species was assigned to Neuroterus (Kieffer 1904). However, the presence of an indistinct suture between the mesoscutum and mesoscutellum could also place the species into Dryocosmus. Whether Neuroterus haasi belong to Drycosmus or Neuroterus must be examined in details, including molecular sequence analysis.

\section{Neuroterus hakonensis Ashmead, 1904}

Lifecycle. Only the asexual generation is known (Monzen 1954).

Geographic distribution. Japan (Monzen 1954).

Host plants. Quercus acutissima (Monzen 1954).

Gall location and structure. Small unilocular, kidney-shaped leaf gall attaches along the midrib of the underside (rarely upperside) of the leaf, small, oblong, yellowish, smooth, with thin, hard wall, $2 \mathrm{~mm}$ in length (Fig. A70) (Monzen 1954).

Phenology. Adults emerge in July (Monzen 1954).

Comments. Sequences of three molecular markers (cytb, ITS2, D2 28S region) showed to be identical with those obtained for L. stirps, thus $N$. hakonensis might be well the alternate asexual generation of L. stirps (unpublished data).

\section{Neuroterus moriokensis Monzen, 1954}

Lifecycle. Alternating sexual and asexual generations are known (Yukawa \& Masuda 1996). The sexual generation was described by Monzen (1954), later Yukawa \& Masuda (1996) established the asexual generation.

Geographic distribution. Japan (Monzen 1954).

Host plants. Quercus mongolica subsp. crispula, Q. serrata (Monzen 1954).

Gall location and structure. Sexual generation catkin galls are small, irregular in shape, yellowis brown, covering with very short hairs, about $2 \mathrm{~mm}$ (Monzen 1954). The asexual generation galls are in buds (Figs A179-180).

Phenology. Sexual generation catkin galls mature in May; adults emerge in May (Monzen 1954).

\section{Neuroterus orbis Monzen, 1954}

Lifecycle. Only the sexual generation is known (Monzen 1954).

Geographic distribution. Japan (Monzen 1954).

Host plants. Quercus serrata (Monzen 1954). 
Gall location and structure. Leaf gall, sprouting small young leaf becomes gall which is rounded, brownish, 2-3 $\mathrm{mm}$ in diameter, unilocular, with sparse surface hairs (Fig. A181) (Monzen 1954).

Phenology. Adults emerge in June (Monzen 1954).

\section{Neuroterus sculpturatus Pujade-Villar \& Wang, 2016}

Lifecycle. Only the sexual generation is known (Pujade-Villar et al. 2016).

Geographic distribution. China (Shaanxi) (Pujade-Villar et al. 2016).

Host plants. Quercus variabilis (Pujade-Villar et al. 2016).

Gall location and structure. Galls in clusters on the leaf edge, with 3-5 galls in each cluster; individual galls are soybean-shaped, $0.5-1.0 \mathrm{~cm}$ in diameter. Young galls are integral and pale green, becoming dark green, and turning brown and detachable when mature. Individual galls are hollow-like, with a tough and thin woody 3.0-4.0 $\mathrm{mm}$ thick wall; the interior space with a single, free-rolling larval chamber (Pujade-Villar et al. 2016).

Phenology. Galls mature in mid-May; adults emerge in early June (Pujade-Villar et al. 2016).

\section{Plagiotrochus Mayr, 1881}

\section{Plagiotrochus follioti Pujade-Villar \& Melika, 2009}

Lifecycle. Only the asexual generation is known (Melika et al. 2009).

Geographic distribution. Nepal (Phulcoki and Siwapuri Dara, 2600 and $2450 \mathrm{~m}$ a.s.1., respectively) (Melika et al. 2009).

Host plants. Unknown.

Gall location and structure. Unknown.

Phenology. Aduls were collected in October (Melika et al. 2009).

\section{Plagiotrochus glaucus Melika \& Tang, 2011}

Lifecycle. Only the asexual generation is known (Tang et al. 2011b).

Geographic distribution. Taiwan (Nantou County) (Tang et al. 2011b)

Host plants. Quercus glauca (Tang et al. 2011b).

Gall location and structure. The gall is a swelling of branches and twigs, usually located in the middle of the twig, never apically; sometimes swellings develop at joints of twigs. The gall is up to $6.0 \mathrm{~cm}$ long, $1.2-1.5 \mathrm{~cm}$ in diameter, coloured the same as the bark of twigs. When the gall mature, tissues hard, lignified. Larval chambers (up to $14 \mathrm{~mm}$ in length and 3-5 $\mathrm{mm}$ in diameter) arranged perpendicularly to main axis of gall, radiating from center of twig toward gall surface, reaching to 2-3 mm from surface (Figs A182-183) (Tang et al. 2011b).

Phenology. Galls mature in late October; adults emerge in the following spring (Tang et al. 2011b).

\section{Plagiotrochus indochinensis Abe, Ide, Konishi \& Ueno, 2014}

Lifecycle. Only females are known (Abe et al. 2014b).

Geographic distribution. Vietnam (Lam Dong Province) (Abe et al. 2014b).

Host plants. Quercus helferiana A.DC. (Quercus subgenus Cyclobalanopsis) This plant species is considered to be the host plant because the holotype female was observed to insert its ovipositor into a young sprout of this evergreen oak (Abe et al. 2014b). 
Gall location and structure. Unknown.

Phenology. Adults emerge in March (Abe et al. 2014b).

Plagiotrochus masudai Ide, Wachi \& Abe, 2010

Lifecycle. Alternating sexual and asexual generations are known (Yukawa \& Masuda 1996).

Geographic distribution. Japan (Honshu, Shikoku, Kyushu) (Ide et al. 2010, Yukawa \& Masuda 1996)

Host plants. Quercus glauca (Yukawa \& Masuda 1996, Ide et al. 2010).

Gall location and structure. Asexual generation galls are in somewhat swollen twigs, with oval larval chambers inside, $1.0-1.5 \mathrm{~mm}$ in diameter and $2.5-3.0 \mathrm{~mm}$ in length each, aggregated but separated from one another by yellowish brown or brown wooden wall (Ide et al. 2010). Sexual generation galls are in buds, its apical end slightly curved, but almost indistinguishable from the healthy bud on the basis of the outline. One to five larval chambers, $2.0 \mathrm{~mm}$ in diameter each, separated from one another by brownish yellow, thin and firm wall at the base of bud and visible by removing bud scales (Ide et al. 2010).

Phenology. Adults of the sexual generation emerge in April; females wasp lays eggs in elongating shoots but galls are not induced in the first year. In the second year, the affected twigs become slightly swollen and the larvae develop in the latter half of the year. In May or June of the third year, the adults of the asexual generation emerge from the swollen twig and lay eggs in juvenile buds. The larvae then begin their development in summer and hibernate as larvae or pupae. In the spring of the fourth year, the adults of the sexual generation emerge from the bud galls and the cycle is repeated (Yukawa \& Masuda 1996, Ide et al. 2010).

Plagiotrochus semicarpifoliae (Cameron, 1902)

Synonyms. Callirhytis semicarpifoliae Cameron, 1902; Plagiotriochus semicarpifoliae: Bellido et al. (2000).

Lifecycle. Only the asexual generation is known (Cameron 1902, Bellido et al. 2000).

Geographic distribution. NW Himalayas (Bellido et al. 2000).

Host plants. Quercus semecarpifoliae Smith (Cameron 1902, Mani 2000).

Gall location and structure. Galls are in acorns (Cameron 1902).

Plagiotrochus smetanai Melika \& Pujade-Villar, 2009

Lifecycle. Only the asexual generation is known (Bellido et al. 2000, Melika et al. 2009).

Geographic distribution. Nepal (Phulcoki, $2600 \mathrm{~m}$ a.s.l.) (Melika et al. 2009).

Host plants. Unknown.

Gall location and structure. Unknown.

Phenology. Aduls emerge in October (Melika et al. 2009).

Plagiotrochus tarokoensis Tang \& Melika, 2016

Lifecycle. Only the sexual generation is known (Tang et al. 2016b).

Geographic distribution. Taiwan (Hualien County). The host-plant Quercus tarokoensis is endemic to Taiwan, so it is possible that $P$. tarokoensis also a Taiwanese endemic (Tang et al. 2016b). 
Host plants. Q. tarokoensis Hayata (Quercus subgenus Quercussection Cerris) (Tang et al. 2016b).

Gall location and structure. Integral unilocular leaf galls. The gall is small, yellowish-white pimple on midribs or lateral veins of young leaves (Fig. A184). The mature gall is 1.6-2.6 mm in length, 0.8-1.0 mm in width (Tang et al. 2016b).

Phenology. Galls mature in late March; adults emerge in March (Tang et al. 2016b).

\section{Trichagalma Mayr, 1907}

Trichagalma acutissimae (Monzen, 1953)

Synonyms: Aphelonyx acutissimae Monzen, 1953; Trichagalma acutissimae: Melika et al. (2010); Trichagalma glabrosa Pujade-Villar, 2012 (synonym in Wang et al. (2016a)).

Lifecycle. Alternating sexual and asexual generations are known (Wang et al. 2016a).

Geographic distribution. Japan, China, South Korea, Taiwan (Yukawa \& Masuda 1996, Melika et al. 2010, Pujade-Villar \& Wang 2012).

Host plants. Quercus acutissima, Q. variabilis (Yukawa \& Masuda 1996, Melika et al. 2010).

Gall location and structure. The asexual gall is spherical, smooth, 5.0-7.0 $\mathrm{mm}$ in diameter, pale yellow, red or brownish-red with minute bark spots, unilocular, with larval chamber in the center, located on veins of both sides of the leaf of (Yukawa \& Masuda 1996; Fig. A69). The sexual galls, scattered or gathered, are on the bracts of staminate flowers in the central stem of inflorescences, especially on the apically-located staminate flowers. Each gall has a single larval chamber, almost spherical, 1-2 mm in diameter (Wang et al. 2016a).

Phenology: Asexual galls begin to appear in early June; asexual adults emerge in late November to December (Yukawa \& Masuda 1996, Melika et al. 2010) and lay eggs in catkin buds; sexual adults emerge in spring (Wang et al. 2016a).

Trichagalma formosana Melika \& Tang, 2010

Lifecycle. Only the asexual generation is known (Melika et al. 2010).

Geographic distribution. Taiwan (Taoyuan, Hsinchu, Taichung, Nantou Counties), Japan (Melika et al. 2010).

Host plants. Quercus acutissima, Q. variabilis (Melika et al. 2010).

Gall location and structure. Galls may be found singly or in groups, most commonly on lateral buds of young shoots. Solitary galls are almost spherical, while closely clustered galls may be deformed (Fig. A118). Old galls persist on the host tree (Melika et al. 2010). The gall when young is juicy, soft, covered with small raised tubercles, and green with purple spots on areas exposed to direct sunlight. The gall when mature reaches $15 \mathrm{~mm}$ in diameter, and is brown with a slightly irregular surface. The mature gall is hollow, with a tough woody wall $1.5-2.5 \mathrm{~mm}$ thick. The interior space contains a single larval chamber, attached to the wall by a stalk, which, however, dry out when the gall is mature. The larval chamber is ovoid, up to $6 \mathrm{~mm}$ in length, and has a tough but thin wall (Melika et al. 2010).

Phenology. Galls appear on the tree from early August; adults emerge in December or might overwinter in the gall and emerge in spring of the following year (Melika et al. 2010). 
Trichagalma serratae (Ashmead, 1904)

Synonyms: Dryophanta serratae Ashmead, 1904; Trichagalma drouardi Mayr, 1907; Trichagalma serratae: Monzen (1929); Neuroterus serratae: Abe (2006); Trichagalma serratae: Melika et al. (2010).

Lifecycle. Alternating sexual and asexual generations are known (Abe 1992, 2006, Yukawa \& Masuda 1996).

Geographic distribution. Japan (Honshu, Shikoku and Kyushu), China and South Korea (Abe 2006, Abe et al. 2007, Melika et al. 2010).

Host plants. Quercus acutissima, Q. variabilis (Melika et al. 2010).

Gall location and structure. Sexual generation gall is a single-chambered gall, irregularly globulous with a thin wall, smooth, tinged with yellow or red, on the surface of the catkins; solitary or several galls clustered per catkin; maximum diamtere $2-3 \mathrm{~mm}$. The asexual gall usually is coalesced but often separate, roughly spherical, closely covered with spines, light green at the appearance, turning fulvous, 10-20 mm in diameter (Figs A185-186). The larval chamber single with an air space between this and the outer woody gall wall, ovoid, attached by its base to the gall wall, maximum $5 \mathrm{~mm}$ in diameter (Abe 2006).

Phenology. Sexual generation galls mature in April-May; adults emerge in May. Asexual galls appear in August; pupation take place in the following September. Some asexual larvae prolong their larval stage for one year (Yukawa \& Masuda 1996).

Trigonaspis Hartig, 1840

Trigonaspis nephroideus (Kovalev, 1965)

Synonyms. Neoneuroterus nephroideus Kovalev, 1965; Trigonaspis nephroideus: Melika \& Abrahamson (2002).

Lifecycle. Only the sexual generation is known (Kovalev 1965, Melika 2012).

Geographic distribution. Far East Russia (Kovalev 1965, Melika 2012).

Host plants. Quercus dentata (Kovalev 1965).

Gall location and structure. Galls are in buds. Infested buds usually enlarged, with 5-7 larval chambers inside (Kovalev 1965).

Phenology. Adults emerge in late May (Kovalev 1965).

Trigonaspis spumeus (Kovalev, 1965)

Synonyms. Neoneuroterus spumeus Kovalev, 1965; Trigonaspis spumeus: Melika \& Abrahamson (2002).

Lifecycle. Only the sexual generation is known (Kovalev 1965, Melika 2012).

Geographic distribution. Russia (Far East) (Kovalev 1965, Melika 2012).

Host plants. Quercus mongolica (Kovalev 1965).

Gall location and structure. Juicy, spherical bud galls, 5-9 $\mathrm{mm}$ in dimeter, with 6 larval chambers in the center of the gall. Galls are green when growing, turn brown when mature. Usually on young sprouts (Kovalev 1965).

Phenology. Adults emerge in May (Kovalev 1965).

Trigonaspis vernicosus (Kovalev, 1965)

Synonyms. Neoneuroterus vernicosus Kovalev, 1965; Trigonaspis vernicosus: Melika \& Abrahamson (2002).

Lifecycle. Only the adult females are known (Kovalev 1965). 
Geographic distribution. Russia (Far East) (Kovalev 1965, Melika 2012).

Host plants. Quercus dentata (Kovalev 1965).

Gall location and structure. Unknown.

Phenology. Females were caught ovipositing into buds (Kovalev 1965).

\section{Ussuraspis Kovalev, 1965}

Ussuraspis nervosa Kovalev, 1965

Synonyms: Ussuraspis nervosa Kovalev, 1965; Trigonaspis nervosus: Melika \& Abrahamson (2002); Ussuraspis nervosa: Melika (2012).

Lifecycle. Only the asexual generation is known (Kovalev 1965, Melika 2012).

Geographic distribution. Russia (Far East) (Kovalev 1985, Melika 2012).

Host plants. Quercus mongolica (Kovalev 1965).

Gall location and structure. Unilocular small shiny elongated leaf galls, often gregarious, attached to the split midrib along the gall's long axis (Figs A187-188); single galls located on the leaf edge usually are rounded, 2.0-3.5 $\mathrm{mm}$ in diameter (Kovalev 1965).

Phenology. Galls mature in August; adults emerge in December (Kovalev 1965).

Similar galls. Galls are similar to those induced by the asexual generations of the westernpalearctic species, Pseudoneuroterus saliens (Kollar) and Neuroterus anthracinus (Curtis). 

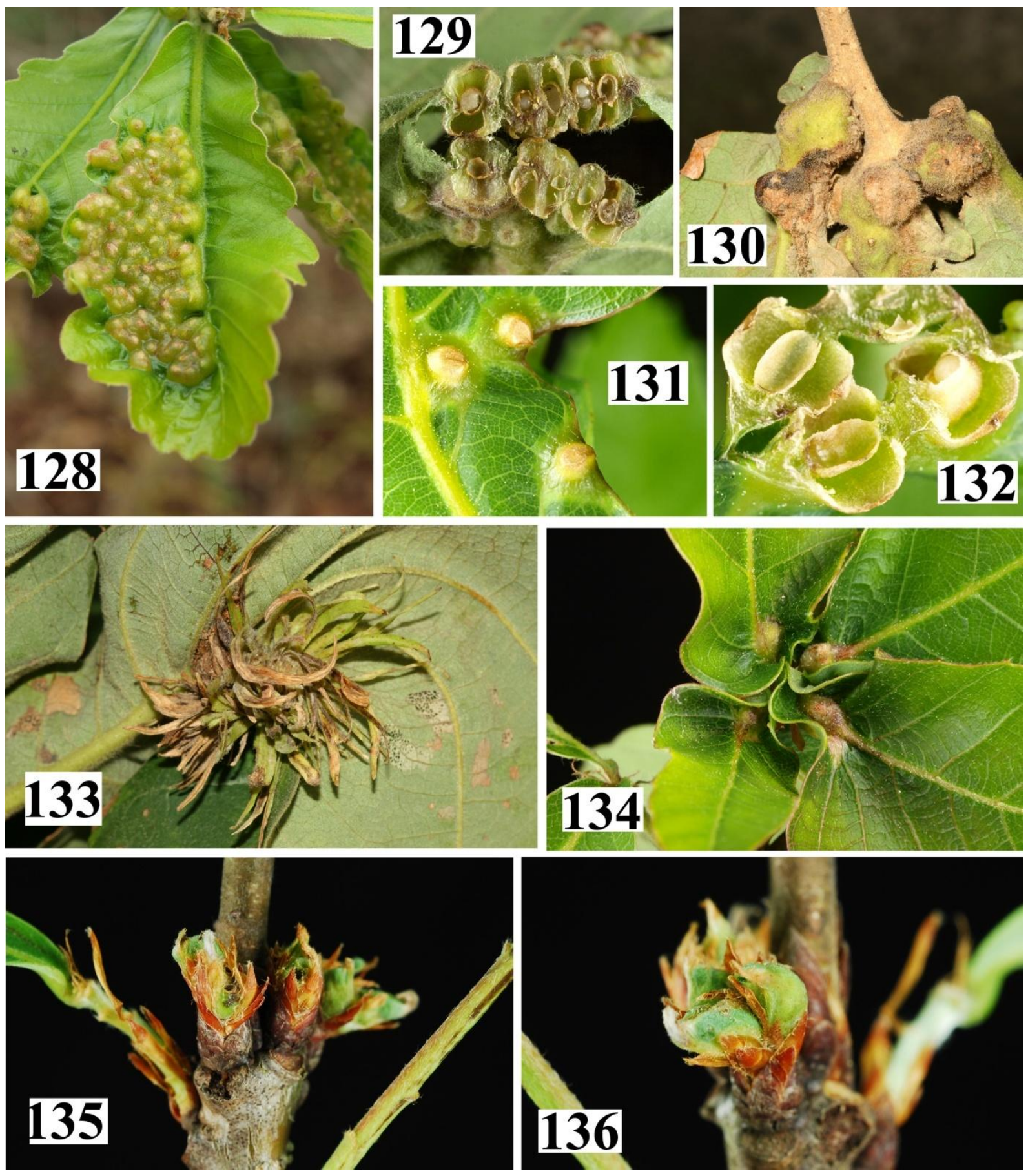

FIGURES A128-A136. Eastern Palearctic oak galls: 128-129, Andricus formosanus, sexual galls: 128, general appearance, 129, dissected galls with larval chambers. 130, Andricus hakonensis, sexual galls. 131-132, Andricus pseudocurvator, sexual galls: 131, general appearance, 132, dissected galls with larval chambers. 133, Andricus pseudoflos, asexual gall, 134-136, Andricus songshui, sexual galls: 134, galls on developed leaves, 135, 136, galls on bursting leaves. 

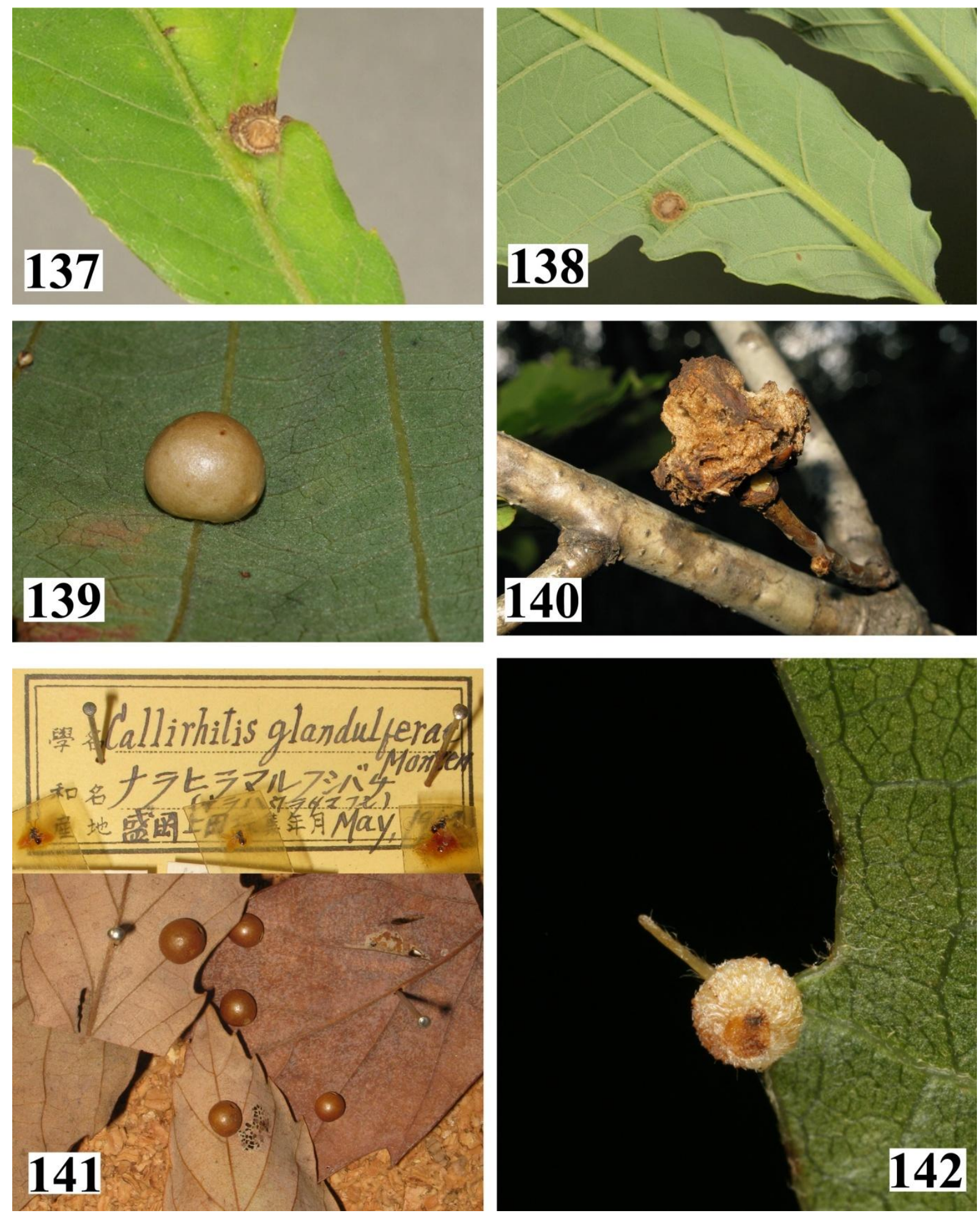

FIGURES A137-A142. Eastern Palearctic oak galls: 137-138, Andricus xishuangbannaus, sexual galls: 137, gall from upper side of leaf, 138, gall from under side of leaf. 139, Belizinella gibbera, asexual gall. 140, Biorhiza nawai, sexual gall. 141, Callirhytis glanduliferae, 142, Cerroneuroterus folimargo, asexual gall. 

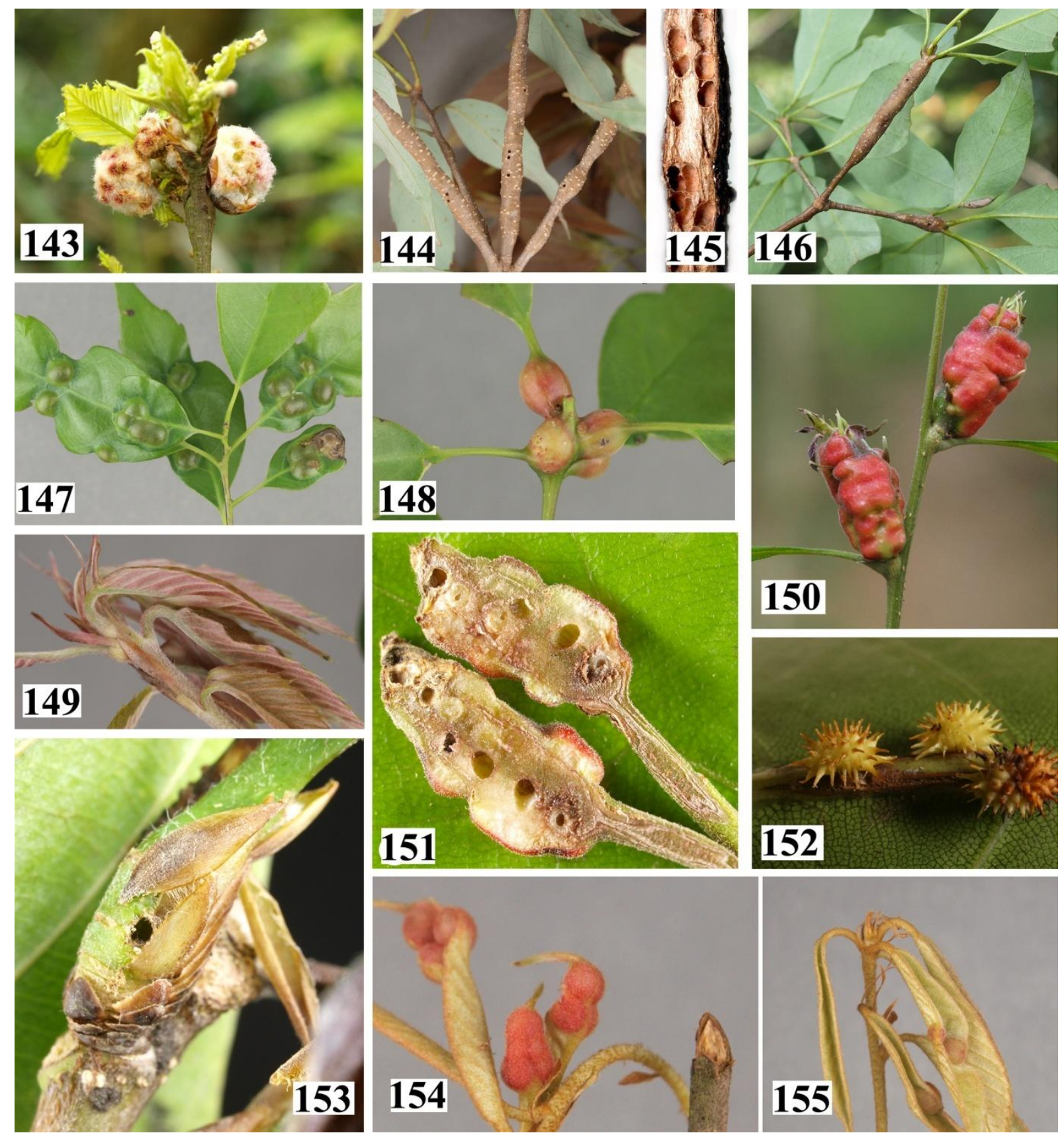

FIGURES A143-A155. Eastern Palearctic oak galls: 143, Cerroneuroterus vonkuenburgi, sexual galls, 144-145, Cyclocynips uberis, asexual gall: 144, general appearance, 145, dissected gall. 146, C. tumorvirgae, asexual gall, general appearance. 147-148, Cycloneuroterus abei, sexual galls. 149, C. ergei, sexual gall. 150-152, C. formosanus, sexual galls: 150, sexual gall, general appearance, 151, dissected sexual gall, 152, asexual galls. 153, C. fortuitusus, sexual gall. 154-155, C. gilvus sexual galls. 

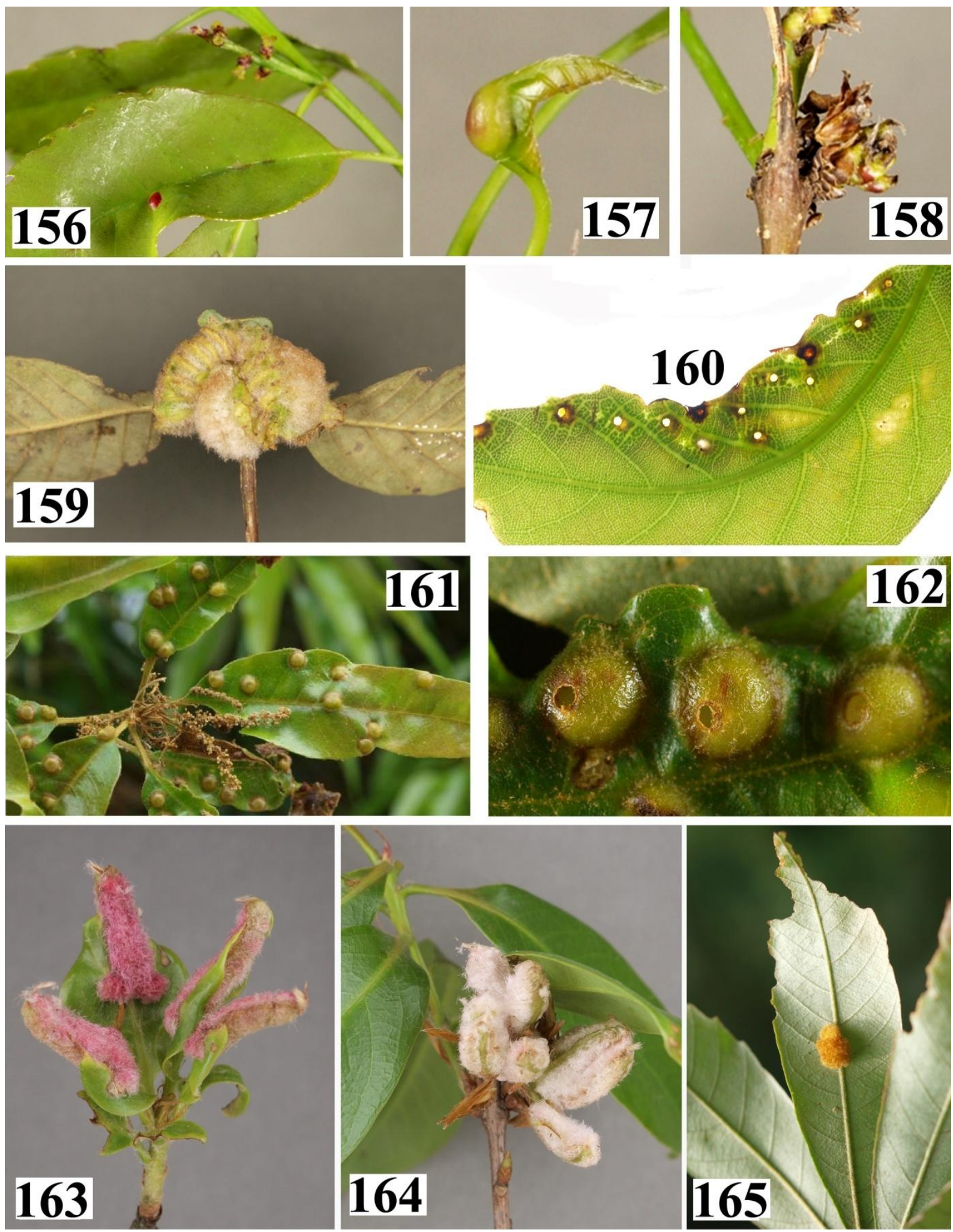

FIGURES A156-A165. Eastern Palearctic oak galls: 156-158, Cycloneuroterus globosus, sexual galls. 159, C. jianwui, sexual gall. 160, C. lilungi, sexual galls. 161-162, C. lirongchinea, sexual galls. 163-165, C. lohsei: 163-164, sexual galls, 165, asexual gall. 

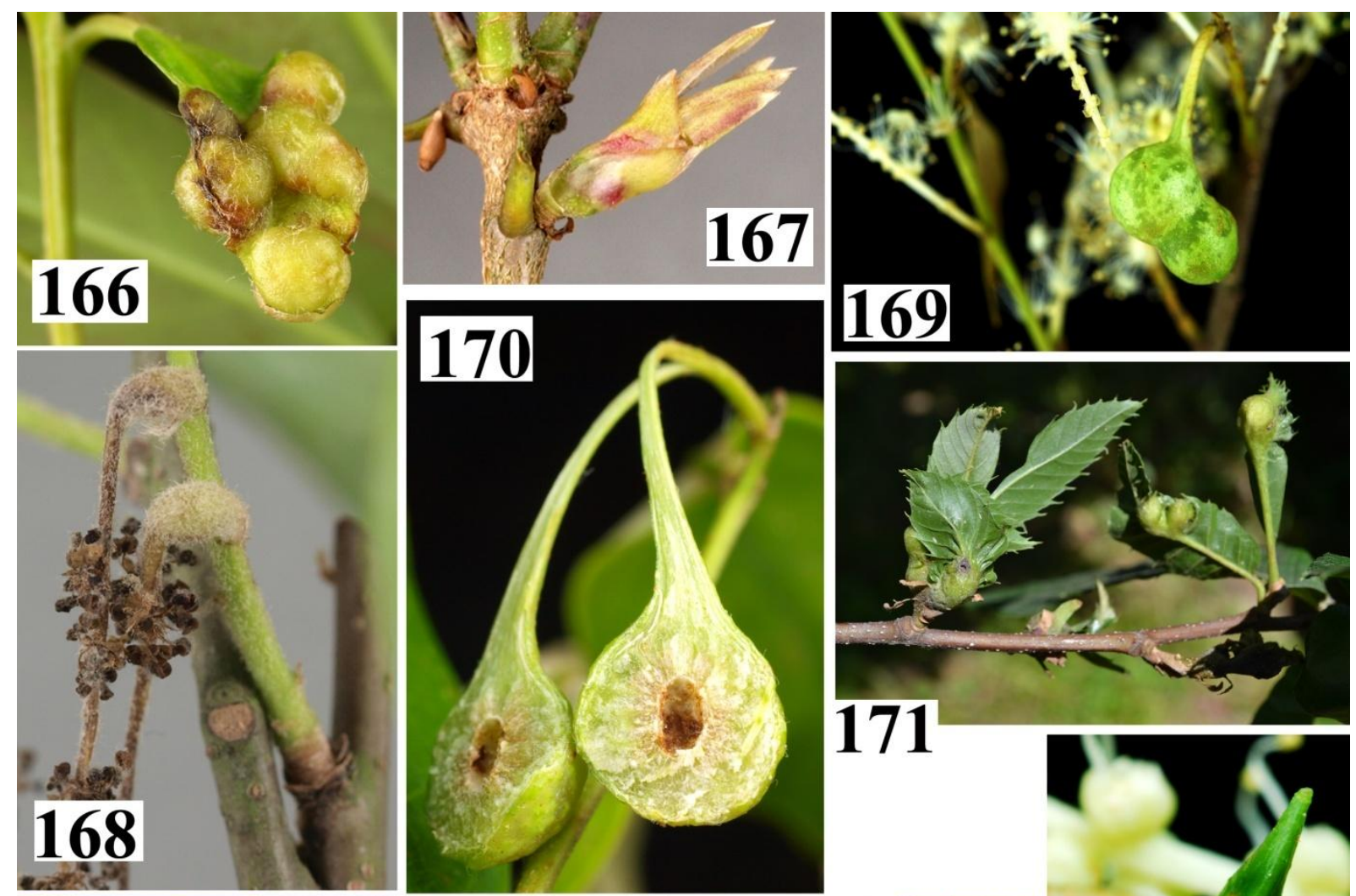

171
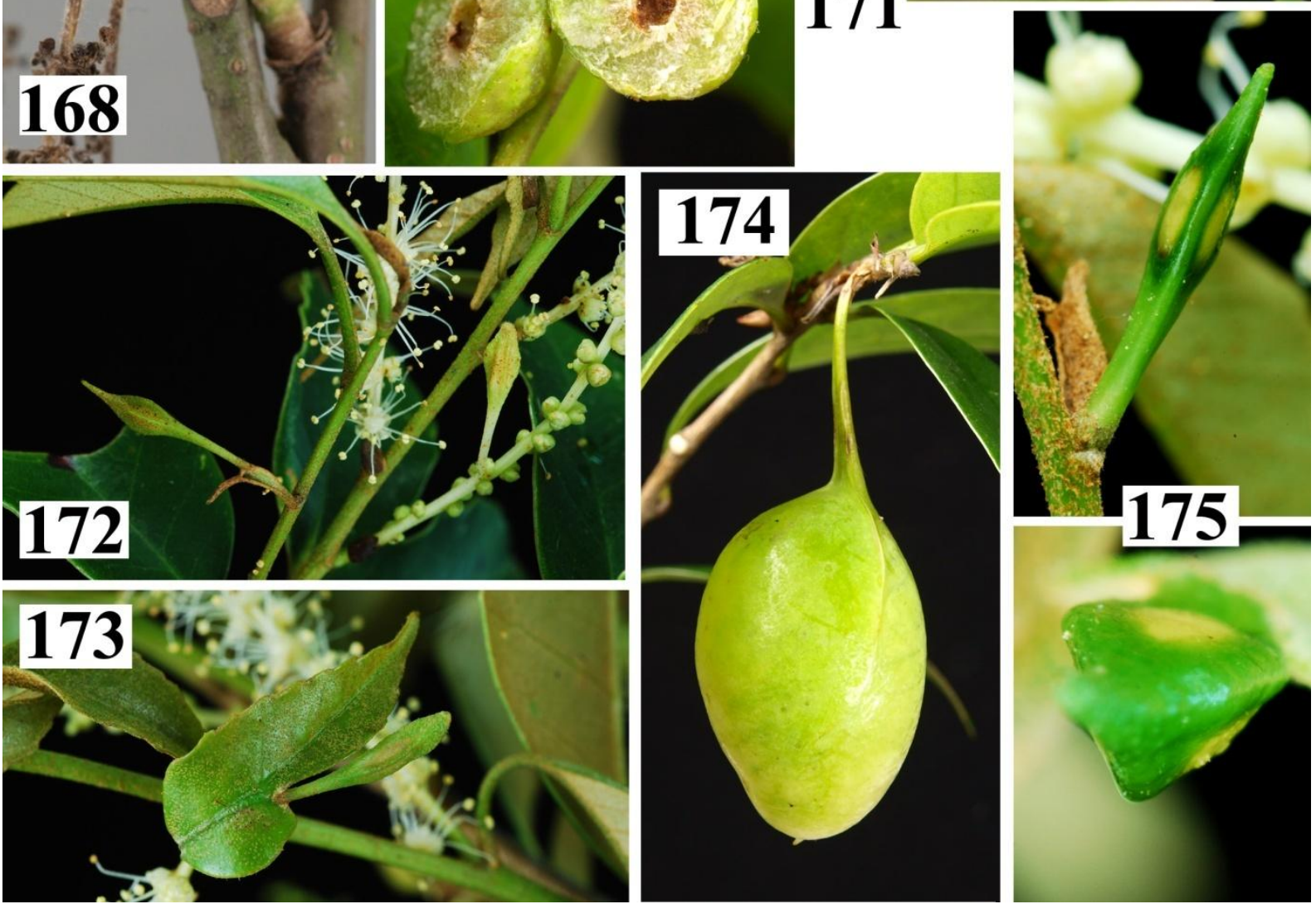

FIGURES A166-A175. Eastern Palearctic oak galls: 166, Cycloneuroterus longinuxus, sexual galls. 167, C. tumiclavus, sexual galls. 168, C. uraianus, sexual galls. 169-170, Dryocosmus carlesiae, sexual galls: 169, general appearance, 170, dissected gall. 171, D. kuriphilus asexual galls. 172-173, D. pentagonalis, sexual galls: 172, gall on catkin, general view, 173, gall on leaf. 174, D. testisimilis, sexual gall. 175, D. triangularis, sexual gall. 

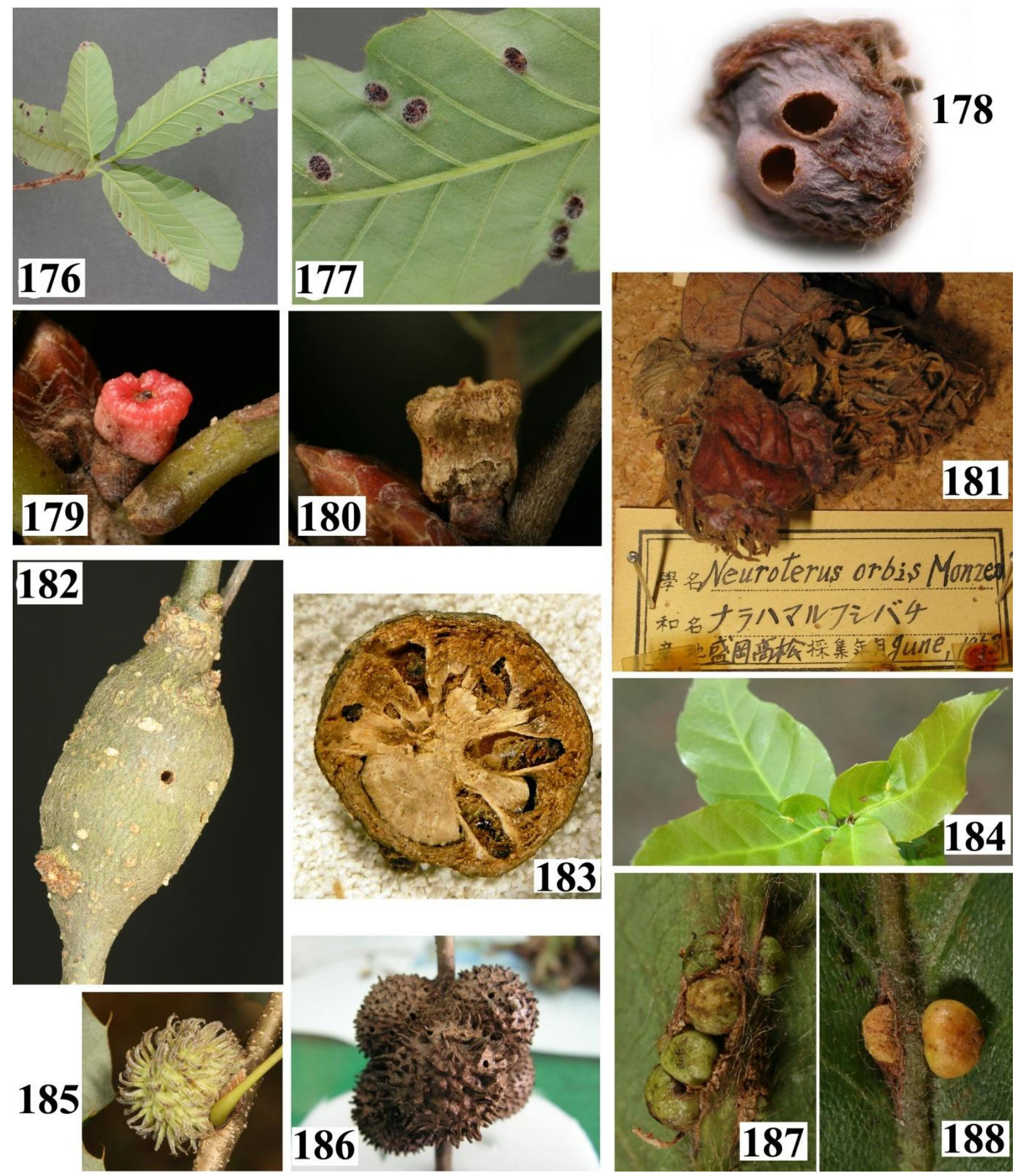

FIGURES A176-A188. Eastern Palearctic oak galls: 176-177, Latuspina manmiaoyangae, sexual galls: 176, general appearance, 177, close-up view. 178, L. stirps, sexual gall. 179-180, Neuroterus moriokensis, asexual galls: 179, young developing gall, 180, mature gall. 181, $N$. orbis, sexual gall (taken from Monzen collection). 182-183, Plagiotrochus glaucus, asexual gall: 182, general appearance, 183, cross-section of the gall. 184, P. tarokoensis, sexual generation gall. 185-186, Trichagalma serratae, asexual galls: 185, young developing gall, 185, mature galls. 187-188, Ussuraspis nervosa, asexual galls: 187 , young developing galls, 188 , mature galls. 


\subsection{Eastern Palearctic Cynipini species with uncertain status}

Andricus noliquercicola Shinji, 1938. Status uncertain. Types lost; unplaced species known from Japan and South Korea (Abe et al. 2007).

Andricus testaceipes subsp. japonicus Monzen, 1953. Status uncertain. Only the sexual generation is known from Japan which induces leaf midrib swelling multilocular yellowish galls up to $5 \times 8 \mathrm{~mm}$, which are protruding on the underside of the leaves on $Q$. serrata; adults emerge in June (Monzen 1953). The galls resembles those of Andricus testaceipes Hartig, 1840 known from the Western Palearctic, however, the wasps differ from those of A. testaceipes and in fact the name was given basis solely on the similarity in gall structure (Monzen 1953). In the Monzen collection one female and one male Andricus were found. Both specimens belong to the Andricus genus. A new name must be given, however, this species might be well synonymic to A. mukaigawae, sexual generation; so types must be revised.

Aphelomyx [sic!] crispulae Mukaigawa, 1920. Status uncertain. Mukaigawa (1920a) described the morphology of sexual generation wasps and their galls on $Q$. mongolica subsp. crispula $(=Q$. crispula). On the basis of the adult's description the wasps are Synergus (inquilines) rather than Andricus (gall inducers). However, the descriptions are not detailed enough to make a definite conclusion. The type lost thus, we treat it as an unplaced species.

Aphelomyx [sic!] glanduliferae Mukaigawa, 1920. Status uncertain. Based on field observations, Mukaigawa (1920b) demonstrated that this species has alternating generations and described the sexual generation wasps and galls of both generations (see galls on pictures on C-127 and C-143 in Yukawa \& Masuda (1996); C-127 is a bud gall, while C-143 is a detachable rounded leaf gall, both on $Q$. serrata. However, the described wasps are more Synergus than Andricus and Mukaigawa probably described an inquiline. However, the descriptions are not detailed enough to make a definite conclusion. The type lost thus, we treat it as an unplaced species

Callirhytis kunugicola Shinji, 1943. Status uncertain. Japan. The types lost. Unplaced species (Abe et al. 2007). In Yukawa \& Masuda (1996) it is depicted on C-083. According to the photo, the gall looks like more a dipteran gall-midge rather than a gallwasp.

Callirhytis tobiiro Ashmead, 1904. Status uncertain. Japan. On the basis of the original description this species is not a Callirhytis; it is more an asexual female of Andricus. The type must be revised (Abe et al. 2007).

Dryophanta japonica Ashmead, 1904. Status uncertain. The type must be revised. Japan. Erroneously transferred to Dryocosmus (Melika \& Abrahamson 2002). Described on the basis of a single female specimen. On the basis of the description this could be a Cynips sexual female.

Dryophanta brunneipes Ashmead, 1904. Japan. Described on the basis of two females from Japan; status uncertain (Ashmead 1904). The type must be revised. 